\title{
Systems Study on Engineered Barriers: Barrier Performance Analysis
}

\author{
Technical Report
}

September 1980

R. T. Stula

T. E. Albert

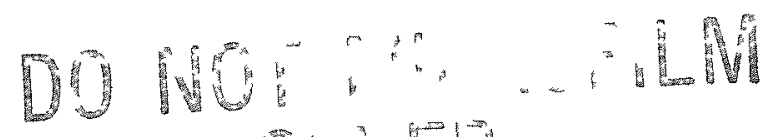

B. E. Kirstein

D. H. Lester

Science Applications, Inc.

1200 Prospect Street

La Jolla, CA 92037

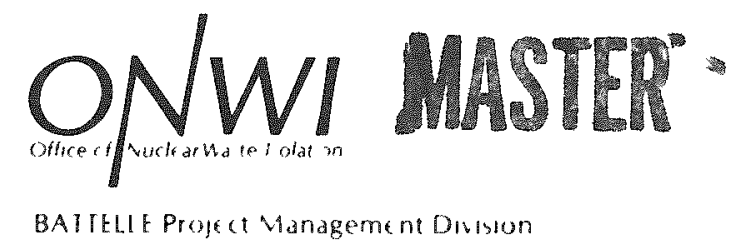




\section{DISCLAIMER}

This report was prepared as an account of work sponsored by an agency of the United States Government. Neither the United States Government nor any agency Thereof, nor any of their employees, makes any warranty, express or implied, or assumes any legal liability or responsibility for the accuracy, completeness, or usefulness of any information, apparatus, product, or process disclosed, or represents that its use would not infringe privately owned rights. Reference herein to any specific commercial product, process, or service by trade name, trademark, manufacturer, or otherwise does not necessarily constitute or imply its endorsement, recommendation, or favoring by the United States Government or any agency thereof. The views and opinions of authors expressed herein do not necessarily state or reflect those of the United States Government or any agency thereof. 


\section{DISCLAIMER}

Portions of this document may be illegible in electronic image products. Images are produced from the best available original document. 


\section{BIBLIOGRAPHIC DATA}

Stula, R. T.. T. E. Albert, B. E. Kirstein, and D. H. Lester, 1980. Systems Study on Engineered Barriers: Barrier Performance Analysis, ONWI-211, prepared by Science Applications, Inc. for Office of Nuclear Waste Isolation. Battelle Memorial Institute, Columbus, $\mathrm{OH}$.

\section{NOTICE}

This report was prepared as an account of work sponsored by an agency of the United States Government. Neither the United States Government nor any agency thereof, nor any of their employees, makes any warranty, express or implied, or assumes any legal liability or responsibility for the accuracy, completeness, or usefulness of any information, apparatus, product, or process disclosed, or represents that its use would not infringe privately owned rights. Reference herein to any specific commercial product, process, or service by trade name, trademark, manufacturer, or otherwise, does not necessarily constitute or imply its endorsement, recommendation, or favoring by the United States Government or any agency thereof. The views and opinions of authors expressed herein do not necessarily state or reflect those of the United States Government or any agency thereof.

Printed in the United States of America

Available from

National Technical Information Service

U.S. Department of Commerce

5285 Port Royal Road

Springfield, VA 22161

NTIS price codes

Printed copy: A11

Microfiche copy: A01 
4. -511

\title{
Systems Study on Engineered Barriers: Barrier Performance Analysis
}

\author{
Technical Report
}

September 1980

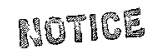

PORTONS OFTHS REPORT ARE ILLEGLEF has been reproduce

R. T. Stula

T. E. Albert

B. E. Kirstein

D. H. Lester

\section{$\checkmark$ DTV ONLE}

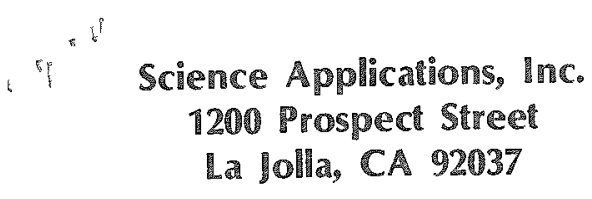

The content of this report is ettecive as of September 1980 This report was prepared bu Science Applications inc undel Subcontract E512-05400 with Battelle Project Management Division. Office of Nuclear Waste liolation under Contract Vo DE-AC06-76-RI O1830-ONWI with the U S Department of Energy This contiact was administered by the Batielle Office of Nuclear Waste Isolation 
Section

Page

ABSTRACT. ..................... 1

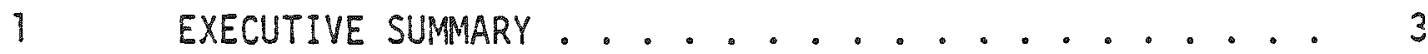

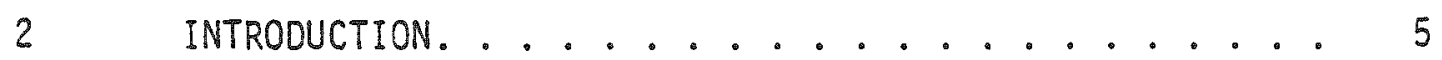

2.1 Previous Studies. ............ 6

2.2 The Modified Model - Overview........ 7

2.3 Scenarios ............. 9

2.4 Alternative Scenarios ......... 9

3 PERFORMANCE MODEL THEORY. .......... 17

3.1 General Description ........... 17

3.2 Temperature Calculations. . . . . . . . 24

3.3 Corrosion ................. 33

3.4 Barrier Failure Criteria. . . . . . . . 40

3.5 Radionuclide Release Rates. . . . . . . 52

3.6 Radionuclide Radiation Fields . . . . . . 75

3.7 Water Repellent Backfills . . . . . . . 87

4 PACKAGE DESIGN DESCRIPTIONS .......... 93

4.1 Previous Work ............ 93

4.2 Cast Stabilizers (Concept E).......... 94

4.3 Heavy Sieeve Packages (Concept Bi)....... 94

4.4 Combination Sleeve/Cast Stabilizers

(Concept BE)............. 94

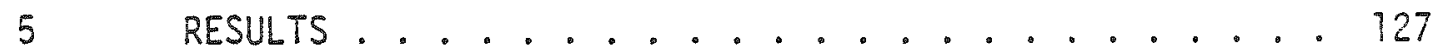

5.1 "Best" Packages from Previous Work. . . . . . 127

5.2 Cast Stabilizer Concept (Concept E) . . . . . 127

5.3 Heavy STeeve Concept (Concept B1) ...... 135

5.4 Heavy Sleeve/Cast Stabilizer Concept

(Concept BE). . . . . . . . 138

5.5 Peak Waste Temperature. . . . . . . . . 138

5.6 Sensitivity Studies........... 143

6 CONCLUSIONS AND RECOMMENDATIONS ......... 151

6.1 Package Performance . . . . . . . . . $15 i$

6.2 Areas of Uncertainty. . . . . . . . 152

6.3 Risk Model Development Recommendation..... 155 
TABLE OF CONTENTS

(Continued)

Section

Page

7

REFERENCES. ................ 159

8

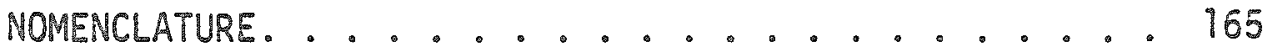

APPENDIX A - "BARIER" PROGRAM LISTING ........ A-1

APPENDIX B - DATA BASE FOR CORROSION MODEL (CORODE) .............. B-1

APPENDIX C - DATA BASE FOR BARRIER FAILURE MODEL

(STRESS) CRITERIA. ......... C-i

APPENDIX D - INPUT/OUTPUT DESCRIPTION ...... D-I

APPENDIX E - SAMPLE PROBLEMS. ........ E-1 
Figure

Page

2-1 Preliminary Waste Package Degradation and Release

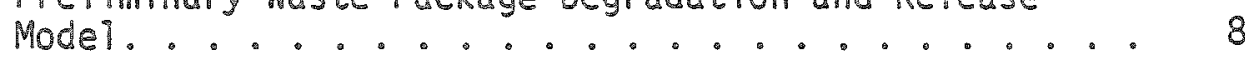

2-2 Proposed Flow Scenario For Flooded Repository. . . . 13

2-3 Values of Dimensionless Pressure on a Transverse

cylinder................................ 14

2-4 Probable Fiow Scenario for Flooded Repository. . . . 15

3-1 PKTEMP Barrier Model ............. 18

3-2 Stylized Waste Package Configuration for Fuel

3-3 BARIER Flowchart................ 20

3-4 PKTEMP Flowchart.............. 30

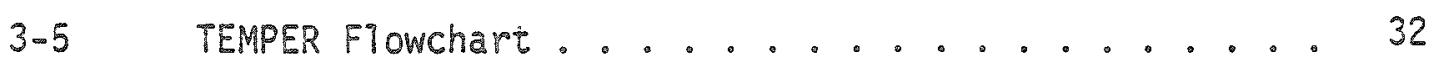

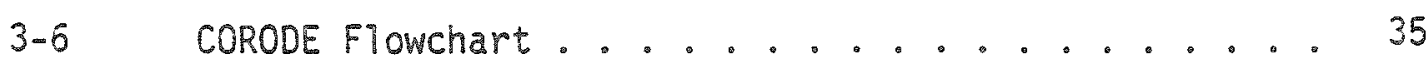

3-7 Composite Baprier Used in Stress Calculations. . . . 4

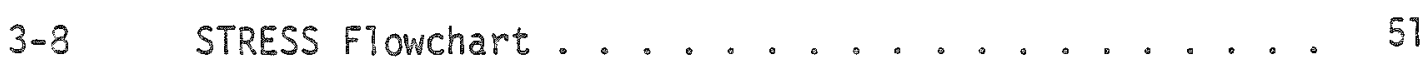

3-9 Illustration of Steady State Solution......... 58

3-10 Steady State Concentration Profiles in a Slab for

3-11 Concentration Profiles for the Approach to Steady

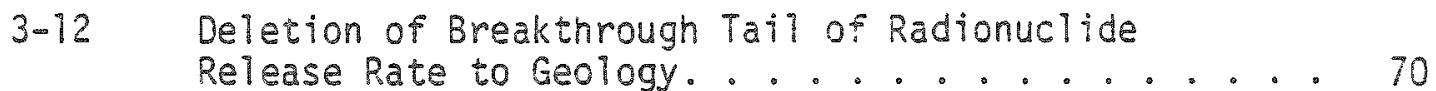

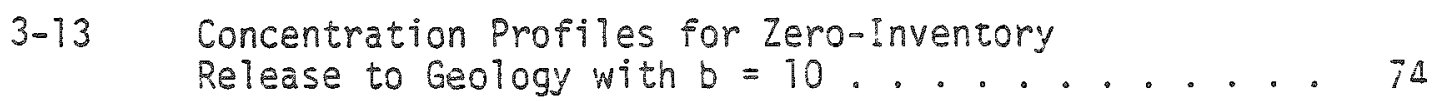

3-14 RELEAS Flowchart ............. 76 


\section{LIST OF FIGURES}

(Continued)

Figure

Page

3-15 Photon Release Rate Vs. Time after Emplacement. . . 82

3-16 Photon Spectrum from Fission Products ....... 83

3-17 Photon Spectrum from Activation Products. . . . . 84

3-18 RADCLC Flowchart............... 90

4-1 Barrier System Conceptual Designs from SURF/SFHP

Program................ 95 


\section{LIST OF TABLES}

Table

Page

3-1 Thermal Loadings Achieved for the Conceptual

Repositories for Once-Through Fuel Cycle. . . . . 25

3-2 Constants for Repository Temperature Calculations . . 27

3-3 Chemical Composition of WIPP-B Salt Brine . . . . 36

3-4 Chemical Composition of Typical Arid Ground Water . . 37

3-5 General Form of CORRAT............. 39

3-6 Retardation of the Transport of Radionuclides Through a Slab Barrier at Steady State for Values of the Radio-Diffusion Parameter, b. ......... 65

3-7 Fuel Composition. . . . . . . . . . . . 77

3-8 Comparison of Radioactivity Content and Heat Generation in Spent Fuel with Prior Data...... 81

3-9 Linear Attenuation Coefficients for Materials . . . 86

3-10 Buildup Factor Parameters at $0.7 \mathrm{MeV}$. . . . . . . 88

3-11 Buildup Factor Material Correspondence. . . . . . 89

3-12 Permeability of Clays and Sand-Clay Mixtures. . . . 92

4-1 Summary of Concepts Studied in FY'79 with Previous Version of "BARIER" Code........ 96

4-2 Summary of Concepts Studied in FY' 80 Follow-on Work Using Previous Version of "BARIER" Code...... 105

4-3 Best Concepts in FY'79 Performance Study. . . . . . . 112

4-4 Summary of Concepts Studied with New Version of "BARIER" Code................ 113

5-1 Comparison of Previous Best Package Designs with Current Results .............. 128

5-2 Previous Best Package Design Results with Current

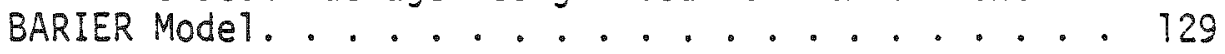




\section{LIST OF TABLES}

(Continued)

Table

Page

5-3 Best Package Designs in Current Study (Oxic

Conditions)................ 132

5-4 Concept $E$ Results in Current Study ....... 133

5-5 Concept BI Results in Current Study. ....... 136

5-6 Concept BE Resuits in Current Study........ 139

5-7 Effect of Backfill Material and Thickness on

Maximum Waste Temperature........... 142

5-8 Effect of Repository Pressure on Package Performance . 144

5-9 Effect of Repository Temperature on Package

Performance. ............. 145

5-10 Effect of Waste Heat Generation Rate on Maximum

Waste Temperature. ........... 146

5-11 Effect of Air Gap Thickness on Maximum Waste

Temperature............... 148

5-12 Effect of Backfill Compaction Coefficients on Net

Pressure on a Barrier at Failure ........ 149

5-13 Effect of Backfill Thickness on Radionuclide

Transport Resistance .......... 150

B-1 Corrosion Rates of Barrier Materials in Brine B. . . B-2

B-2 Corrosion Rates of Barrier Materials in Ground Water. B-3

C-1 Compressive Yield for Carbon Steel ........ C-2

C-2 Compressive Yield for Inconel Alloy $800 \mathrm{H} \ldots \ldots . . . \mathrm{C}-2$

C-3 Compressive Yield for Stainiess Steei Type $304 \ldots$ C-3

C-4 Material Properties................. C-3

C-5 Melting Points Used for Shear Modulus Extrapolation. . C-5

C-6 Temperature Fits for Shear Moduius ....... C.5 
LIST OF TABLES

(Continued)

Table

Page

C-7 Temperature Fits for Bulk ModuTus........... C-6

C-8 Allowable Stresses for Internal Pressure . . . . . C C-6

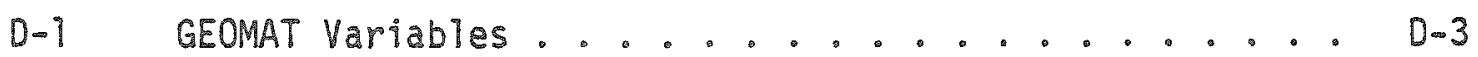

D-2 MATMAT Variables .............. $0-4$

D-3 BARFIL Vartables................ D-5

D-4 General Form of DATSET ............ D-8

D-5 Input Information Required for BARIER Execution.... D-9 
A performance assessinent model for multiple barrier packages containing unreprocessed spent fuel has been modified and applied to several package designs. The objective of the study was to develop information to be used in programmatic decision making concerning engineered barrier package design and development. The assessment model, "BARIER", was developed in previous tasks of the System Study on Engineered Barriers (SSEB). The new version discussed in this report contains a refined and expanded corrosion rate data base which includes pitting, crack growth, and graphitization as well as bulk corrosion. Corrosion rates for oxic and anoxic conditions at each of the two temperature ranges are supplied. Other improvements include a rigorous treatment of radionuclide release after package failure which includes resistance of damaged barriers and backfill, refined temperature calculations that account for convection and radiation, a subroutine to calculate nuclear gamina radiation field at each barrier surface, refined stress calculations with reduced conservatism and various coding improvements to improve running time and core usage. This report also contains discussion of alternative scenarios to the assumed flooded repository as well as the impact of water exclusion backfills. The model was used to assess post repository closure performance for several designs which were all variation of basic designs from the Spend Unreprocessed Fuel (SURF) program. Many designs were found to delay the onset of leaching by at least a few hundreds of years in all geologic media. Long delay times for radionuclide release were found for packages with a few inches of sorption backfill. Release of uranium, plutonium, and americium was assessed. 
9

- 


\section{EXECUTIVE SUMPARY}

This study is concerned with the disposal of unreprocessed fuel elenents in salt, shale, basalt, or granite repositories using a system of engineered barriers in addition to the geologic media as containment. In an earlier study, a scoping model of barrier perfomance was developed and applied to a representative spectrum of barpier designs (Lester, 1979). Results of thdt work suggested additional designs which were evaluated in a subsequent study (Stula, 1980a). This work represents a continuation of previous studies and includes more refined model development in addition to performance evaluation of many barrier package design variations. "Perfurmance" is related to only a maximum individual dose after repository closure and not to other factors such as waste transportation.

Barrier performance is determined in terms of two main parameters: time of initial release of radionuclides to the geosphere (leach begin time) and duration of radionuclide release. Time is measured from a zero time scenario when the repository is sealed and assumed saturated with water. The perfornance model treats a barrier package as a series of layers each consisting of a solid wall(s), filler (or backfill), and a gap between barriers. Materials and designs for barrier packages are chosen to give a range of cost and perfornance. The key concern is to identify where addicional barrier cost yields little increased benefit.

The major performance model refinements performed in this study include addition of radionuclide release (transport) and radiation field calcallation models, improvenent of the temperature gradient and stress calculation nodels, and expansion of the corrosion rate data base. In addition, the possible effects of alternative repository scenarios and the use of water repellant backfills on performance are discussed. All pertinent performance model theory is provided in this report.

Main barrier package design considerations include the effects of external geologic crushing forces and corrosive behavior of the associated high pressure and high temperature brine/water. Evaluation of various general proposed package design concepts (Stula, 1980a) showed a design with a cast solid lead stabilizer to be the most promising. In this design, corrosion 
resistance is the most important factor in determining package life since the voidless package stabilizer is sufficient to withstand geologic crushing forces. Of designs investigated which did not utilize a cast solid stabilizer, a design with a thick corrosion-resistant hole sleeve gave the best performance. Results of this previous study indicated that these two design concepts, or a combination of the two, were nost desirable. Thus, perfomance calculations in this study are limited to design variations of these general concepts. However, evaluation of "best" package designs as determined in previous work is performed in this study with the refined performance model for comparison.

The performance indicates that package lifetimes of at least a few hundreds of years in all geologies can be achieved. Furthermore, judicious use of backfills to sorb radionuclides and or exclude water can reduce radionuclide release after barrier failure as well as delay the onset of radionuclide release. Results indicate that a few inches of backfill thickness are sufficient to supply the necessary barrier to radionuclide release. Large backfill thicknesses are of little advantage as long as sufficient sleeve thicknesses and/or a cast stabilizer are used. The stress defense contribution of the backfill is questionable as it contributes very little and never contributes to stress application if a very "soft" material is used. The key question with regard to backfills remains whether the backfill material will retain its properties or geometry over long periods of time (over 1000 years). There is a serious question that a backfill would be intact in an environment capable of leaching material from a ceranic waste material.

Calculated perfomance results using the refined BARIER model roughly correspond to those from the previous model. While corrosion rates in the new data base tend to be higher than previous values, the stress calculations for geologic crushing forces are based on real failure rather than ASME code criteria which tend to be very conservative. 


\section{INTRODUCTION}

This report describes the work on engineered barrier performance assessment performed by Science Applications, Inc. (SAI) for Pacific Northwest Laboratories (PNL) during the period January 1, 1980 to September 30, 1980. This effort under office of Nuclear Waste Isolation (ONWI) sponsorship was a follow-on to previous studies from June through December 1979. The objective of the work was to develop means to evaluate performance of proposed design concepts, assess the sensitivity of the package performance to specific design parameters and support evaluation of the incentives for use of various types of packages. Results of this study are intended to support decisions by ONWI regarding engineered barrier development plans. Additional studies at PNL on the results of releases to the geosphere complement this work and provide additional decision-making inputs.

The code developed as a part of this work provides a good beginning for a detailed near-field model which would be part of an integrated repository risk assessment model. Technology transfer of this work is underway to support efforts to develop such an integrated model.

Efforts under this study were limited to some specific circumstances. Nevertheless, the model was developed in a manner which allows expansion into many other circumstances. A specific list of candidate materials was used (see later section of this report), a limited set of designs was assessed based on previous conceptual studies (Westerman, 1979), four basic water chemistries were used, specific repository designs based on the GEIS (DOE, 1979) were assumed and one specific scenario (flooded repository) was assumed. The study was restricted to PWR spent fuel storage but is easily extended to other waste foms. The parameters considered were not intended to represent an exhaustive list of possibilities but rather to be a wide ranging list of possibilities which provide a representative sample for the purpose of understanding conceptual burial performance parameter sensitivity. Thus, many excellent material choices and design possibilities have likely not been considered due to deliberate scope limitation. Barrier package development activities will provide information for data base expansion as the model is incorporated into integrated risk assessment 
models. The code will easily accomodate such changes due to modular design and methods of data entry.

While the code used was written specifically for the DEC-10 system, it is composed of standard FORTRAN IV and will run on most machines with minor changes in input/output and file control statements. A user manual has been prepared and issued under separate cover as an interim report (stula, 1980b). The report is a condensed version of this report and intended to provide sufficient code documentation for future users. All of the information pertaining to model theory and development in the interim report is contained in this report.

\subsection{PREVIOUS STUDIES}

The study described in this report represents a follow-on effort to previous studies. The initial work was intended to provide rough assessment to guide further studies. Experience gained in the initial efforts was used to determine where improvements should be made to the model and what additional design concepts should be considered in the follow-on work. The key assumptions, scenario descriptions, and repository designs are the same as reported earlier (Lester, 1979) (Stula, 1980a). The reader is referred to the referenced documents for additional details.

Past assessments focused on some design concepts which appeared to offer lifetimes considerably larger than others. of particular concern was the problem of package crushing in rock masses where creep was significant le.g., salt and some shales). Concepts employing heavy-walled bore hole sleeves and/or cast stabilizers around the spent fuel bundle were found to offer good defenses in high creep geologies. These were further evaluated in this study. other promising concepts from the past studies were also included in this follow-on study. 
THE MODIFIED MODEL-OVERVIEW

The barrier performance model used for the calculation discussed in this report was a modified revision of the model used for previous studies (Lester, 1979) (Stula, 1980a). Extensive modifications have been made. The key changes were

- complete overhaul of corrosion rate data base as a result of expanded literature survey and conversation with various corrosion experts

- addition of a detailed radionuclide release rate model which accounts for resistance from damaged barriers, backfill sorption and diffusion in the backfill

- replacement of ASME code criteria for crushing with a detailed stress model to assess the time of actual plastic yield of a barrier wall under external pressure stress

- refinement of temperature gradient calculation to assess thermal radiation across clearance gaps

- addition of a detailed calculation of nuclear radiation fields at package barriers

Figure 2-1 is a simplified block diagram of the improved model. A more-detailed description and diagram can be found in Section 3.1, General Description.

The current version of the model tends to give failure times which are similar to the old model. This is because the reduction in wall thickness requirement due to less conservative stress treatment is offset by higher corrosion rates in the data base. The higher corrosion rates result from consideration of mechanisms other than bulk corrosion such as pitting, crack growth and graphitization. Radionuclide releases are much more delayed and attenuated than in the old model because sophisticated backfill models are employed which take credit for more sorption and diffusion resistance effects.

Larger time increments are used in the improved code which make running time comparable to the older version. A study of accuracy indicated that large time increments (ten to 100 years) do not significantly affect accuracy within significant figures. Using the DEC-10 computer, it was found that a single package design could be run in one geology and water chemistry (oxic and anoxic) for a few dollars of machine time. This is comparable to experience with the previous version. 


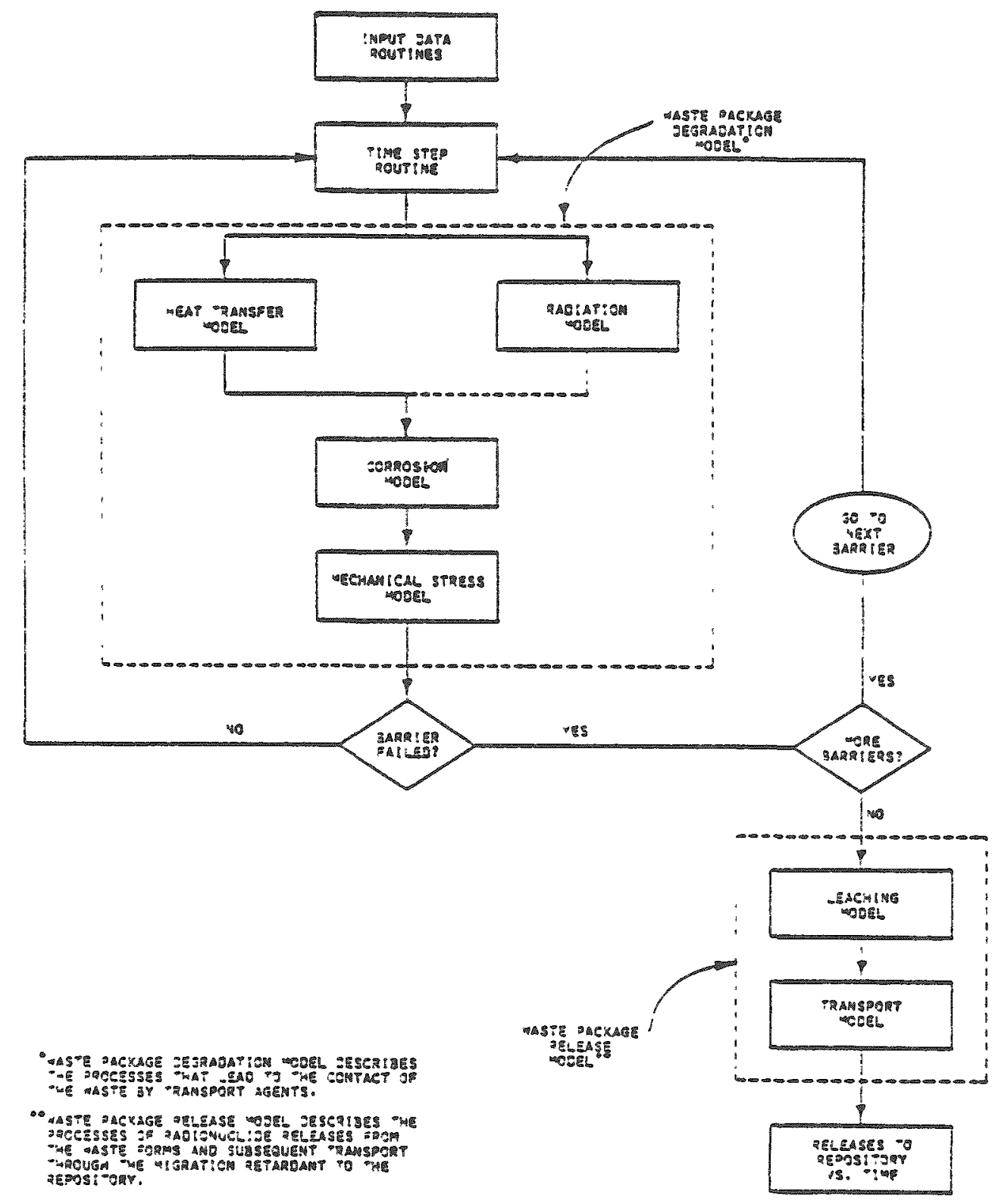

Figure 2-1. Preliminary Waste Package Degradation and Release Model. 
The postrepusitory closure scenario used in this study was the same as that used in previous studies. Like an risk assessment models this one is scenario specific. The scenario considered is a very stringent case for possible package fallure and near-field release.

At time zero the package containing one 6.5 -yeap-old, 3.3 percent enriched, 33,000 MWD/MTHM PWR Fuel bundle is assumed to be immersed in the geologic medium which is saturated with water of appropriate chemistry for the postulated geologic setting. All calculations are carried out assurning both oxic and anoxic chemistry. The ground water chemistry is described in Section 3.3. Sufficient circulation of water is assumed so that there is no build-up of radionuctides in the near-field. This is a conservative (i.e., highest release) assumption. All packages are assumed to experience the same environment and respond in identical fashion.

The model does not account for upset conditions or sensitization of the package barriers from previous events or manufacturing filaws. Probabilities of such deviation could be included in the model in later risk assessment applications.

Some alternative scenarios are discussed in the next section. While the model does not actually consider these, the effect on the results of calculations if they were to be considered is discussed.

\subsection{ALTERMATIVE SCENARIOS}

Scenarios other than the saturated near-field scenario could produce similar or greater consequences. The following alternative scenarios are discussed in this section

- moisture in the near-field in limited quantities with package intact at time $=0$

- dry environment with later water intrusion (limited and unlimited water available!

- direct package intrusion by humans

- repository flooding with very high velocities and large water avallability

- major disruptive natural event such as voicanism or seismic disturbance 
2.4.1 Limited Near-Field Moisture

The "BARIER" code is based on some key assumptions which relate to an unl imited moisture supply. These are

- corrosion proceeds in a manner that would be expected in systems with no significant corrosion product build-up or corrosion agent depletion in the water

- radionuclides are carried away from the package at sufficient rate to make a near-zero concentration of radionuclides in the near-field

- corrosion mechanisms are those expected in a liquid/solid system (no vapor or gas phase)

- the mechanical properties of the backfill are those of a medium saturated with water

- radionuclide sorption and water transport in the backfill is characterized by a porous medium saturated with water

In the event that the water supply is limited then a vapor phase would be present, the backfill would not be saturated and the assumptions above would be invalid.

If such a scenario were assessed the effect on corrosion rates would be reduction due to build-up of corrosion products and depletion of corrosion agents and possibie increase due to vapor phase reactions. Data are lacking to allow a reliable quantitative assessment. It would be expected that corrosion rates would be equal to or less than those used in the current model since build-up of products and reduced corrosion agents (e.g., oxygen) would probably be a larger influence than influences due to introduction of a vapor phase.

In most instances the backfill mechanical properties are greatiy improved when water content is reduced from saturation. Thus, the backfill would offer better defense against crushing in media with high creep.

If the backfill were unsaturated then three effects on radionuclide transport would be expected: (1) reduced sorption because of reduced surface area/moisture contact, (2) reduced flow due to reduction of flow paths and effective moisture conductivity, and (3) reduced discharge rates because of near-field build-up of radionuclide concentration. Effect (1) tends to increase release rate but (2) and (3) greatly reduce release rate. The net effect would likely be reduced release rates, larger release time and longer time to initial release. 
The overall result for this scenario would be expected to compare to "BARIER" results in that the package would last longer before initial leaching of the waste and subsequent release would be more delayed and more spread out in time. This is not surprising because water is the key to package failure and radionuclide transport.

\subsubsection{Dry Environment with Subsequent Water Intrusion}

The same assumptions as mentioned in Section 2.4 .1 are affected and similar effects on the resuits would be expected.

The dry period would introduce a delay time to failure with only the possibility of failure from inadequate design. Experience in archeology shows that ancient, crude metal objects lasted aimost indefinitely in a dry environment. If the package is not adequately designed for forces caused by rock creep, such forces could result in crushing of the package. The wet period would then follow and be different from the model only if the water supply is unlimited as discussed in Section 2.4.1.

\subsubsection{Direct Intrusion}

Direct intrusion may consist of many forms including resource extraction, exploration, or repository exploitation. Direct intrusion introduces mechanisms for damage of the package which are not accounted for in the model. Such intrusion could be considered in package design if reasonable and probabilistic assessment were made of the resultant releases. No relation between this scenario and the one calculated by "BARIER" can be drawn.

\subsubsection{High Velocity Flooding - Large Water Availability \\ Such a scenario could result from a gross breach of the repository} under influence of a pressure gradient or could result from "pumping" in the near-field from themal hydraulic circulation induced by package heat loads. With regard to barrier failure, this would appear no different than the scenario that the "BARIER" code is based on. In terms of radionuclide release, the results would be much different. With large circulation rates the backfill would likely be damaged by erosion and contain flow channels or even disappear from the 
system. If such degradation were simulated the results would be greatly reduced or zero release attentuation after the package was breached and leaching had begun. Leaching rates would then be the same as those observed in inversion leach tests previously cited (Katayama, 1980 ) and the same as those calculated by the old version of BARIER (Lester, 1979).

Frequentiy, in discussions conceming package leaching, a flow scenario such as depicted in Figure $2-2$ is presented. Water flowing past the package in a flood scenario is seen to penetrate on the upstream side, dissolve material, and emerge on the downstream side. Hydrodynamically, this is highly unikely. If it is assumed that the package backfill is imact then the situation is pepresented by flow past a transverse cylinder constructed of a porous solid. Figure $2-3$ shows the dimensionless pressure distribution around such a transverse cylinder for three flow regimes (Schichting, 1960): potential flow, subcritical flow, and supercritical flow. In the case of very slow flow (creeping flow) the potential flow profile would be appropriate. As the Reynolds number increases fincreasing velocityl the flux would proceed through subcritical to supercritical. In potential flow the backside pressure is precisely equal to the frontside pressure (no drag) and there is a low pressure node at the side shoulder. one would then expect a "backwash" toward the node as depicted in Figure $2-4$ if there is any internal circulation. A pure potential flow with no drag will induce no "backwash" but "near-potential" condition would as described. The other flow regimes are similar with the possibility of some circulation as in figure $2-2$ in the subcritical region because the mode is weak and there is some overall pressure differential. However, the subcritical pegion will be highly unstable and subject to boundary layer detachment at the slightest perturbation and go toward the supercritical profile. Note that while the supercritical profile shows an overall pressure difference there is a highiy pronounced "backwash" node. While the pressure distributions presented are for fiow in a large open space around the package (i.e.s ignoring the geology) it seems likely that the whole scenario is not plausible unless a large space has opened up (from catastrophic degradation of the repository as in the case of dissolving away the salt).

The BARIER model assumes flow by diffusion only with no "flow through". The foregoing discussion indicates that flow-through models are probably unrealistic. 


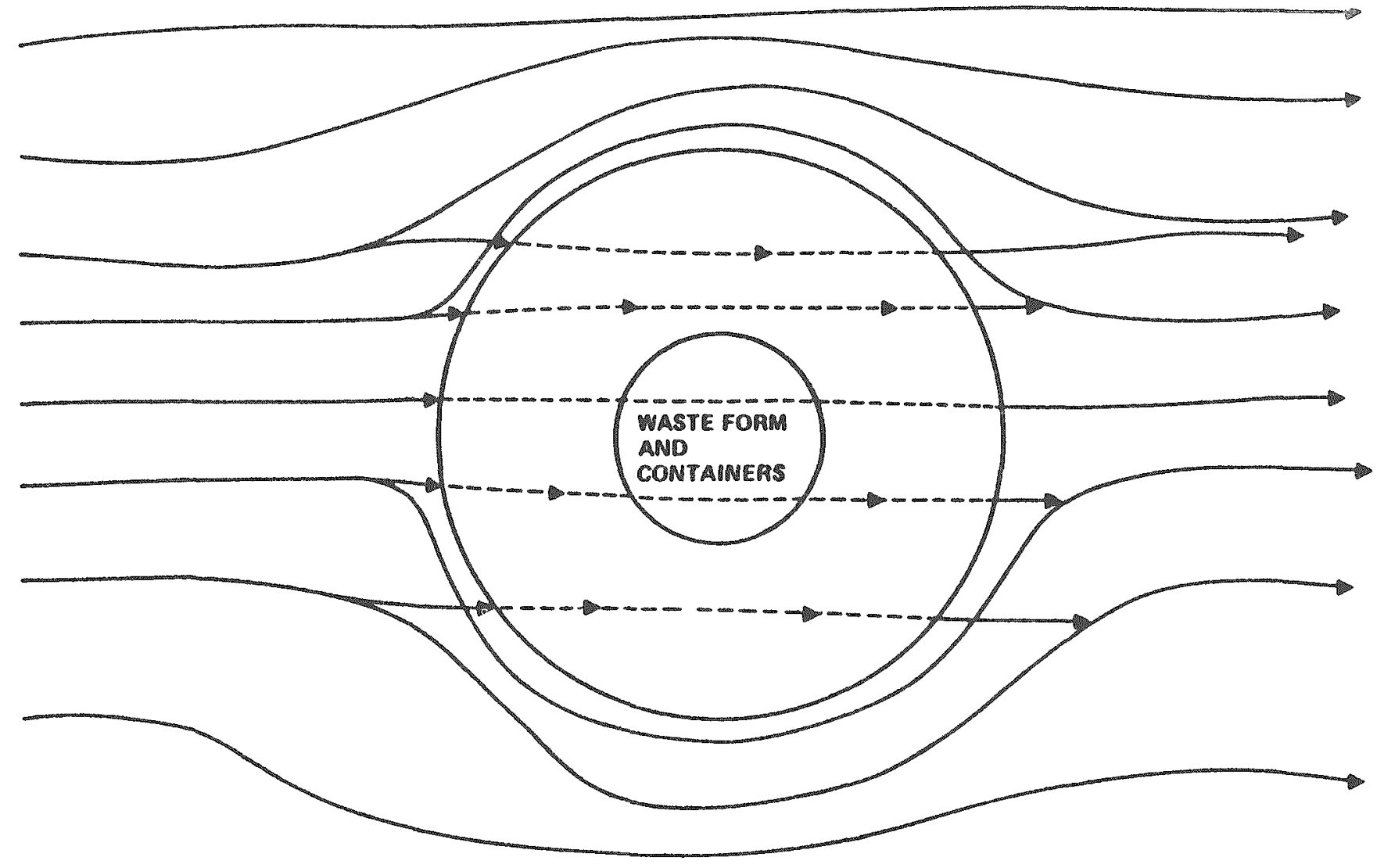

Figure 2-2. Proposed Flow Scenario for Flooded Repository. 


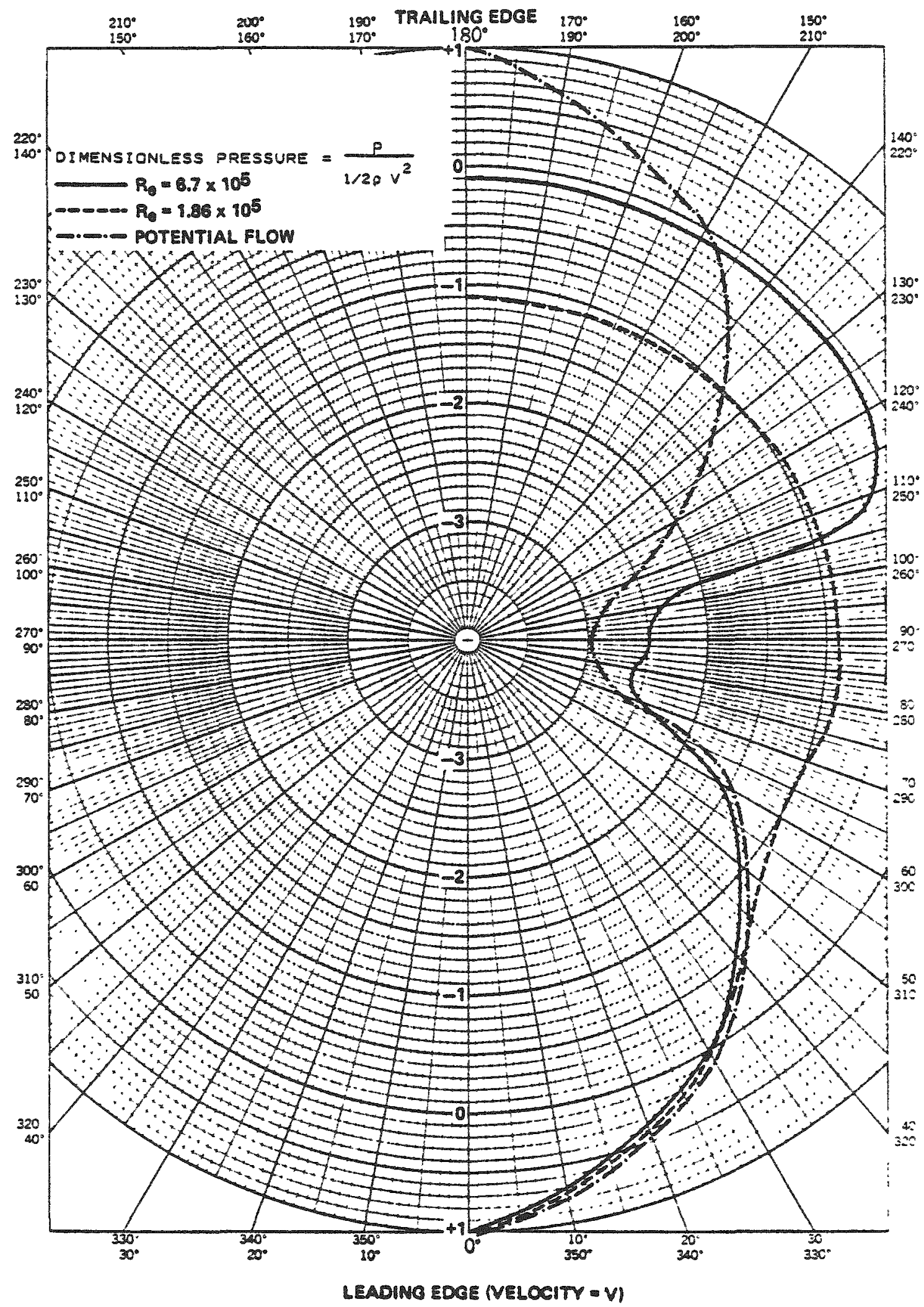

Figure 2-3. Values of Dimensionless Pressure on a Transverse Cylinder. 


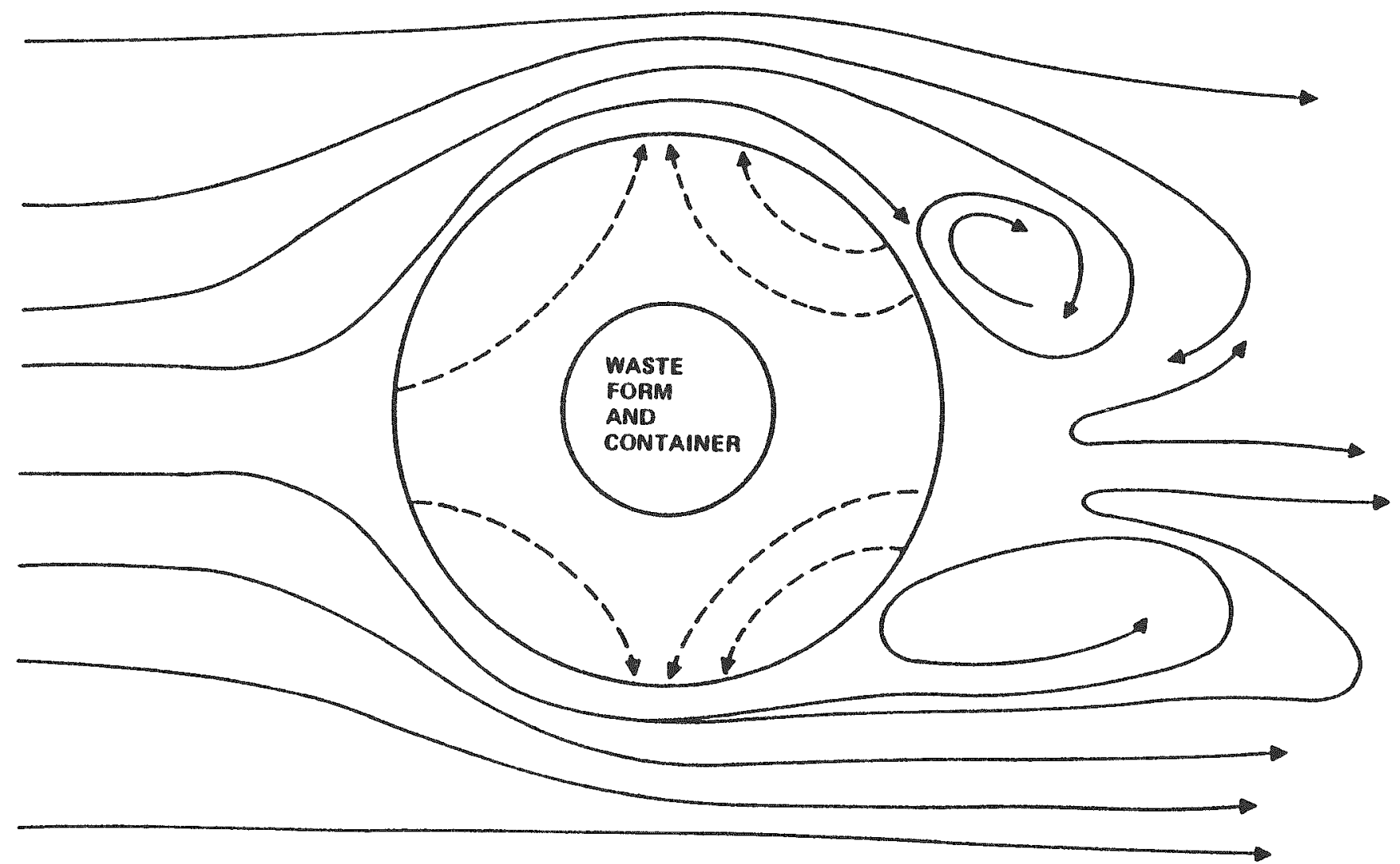

Figure 2-4. Probable Flow Scenario for Flooded Repository. 


\subsubsection{Major Hatural Disruptive Event}

The concept of protection through use of an engineered package probably is not compatibie with major natural seismic events or repository volcanism. The only relation of such scenarios to the "BARIER" model which can be discussed is the effect of seismic activity some distance away which gives attenuated disturbances to the repository. The near-field effect of such a scenario couid be to accelerate degradation by causing vibration damage to the backfill or to the containment vessels. Such damage is oniy significant if water is also present. Therefore, this is a modification of the scenario considered in the code and is a more severe case. Depending on the severity of the disturbance, the package might be breached at a much earlier time or even immediately. Damage to the backfill (cracks, holes, etc.) could reduce the radionuclide retention properties. The result would be earlier and more sharply beaked releas. 


\section{PERFORMANCE MODEL THEORY}

In this section details of the physical theory used in the performance assessment nodel are discussed. The theory subsections gemeraliy pardilel the subroutines used in the BARIER code. Each subsection is designed to supply sufficient detail for clear understanding of the assumptions, model formulation and santents of the data base.

\subsection{GENERAL DESCRIPTION}

The package is viewed as a nulti-layered (barpier) assembly which undergoes a pailure process starting with the outermost barpier and proceeding inward. Each barrier element is envisioned as shown in Figure $3-1$ and together the elements form a package concept shown in Figure $3-2$. (ilots that the number of barriers nay be less than or more than that shown in figure $3-2$ ). The outer material (\$2) of a barier is assumed to possess no structural strength and to act only as a corrosion protector or radiation shieid. The existence of solid wall(s), fillers or jaos in a particular design is conveyed to the nodel by setting the diameters of each barpier layer to the appropriate value. if a particular barpier layer does not evist, then the I. J. of that layer is set equal to the 2. D. The inner sarriers are protected from corrosive attack and from extemal forces by the outer barriers. as ach barpier fails the next inner barrier is subjected to the water environent and the resository pressure/temperature conditions.

Fijure $3-3$ snows how the nodel assesses the successibe fillure and attack of the barriers which lead to leaching and rationuclide release after failure of the last barrisr.

Initially a heat transfor model is used to detarmine the maximum steady-state temperature that ine wasts would attain if the oackage renained intact in a repository at its naximum temoeratura. If a tenderature of $53^{\circ}$ $\left(380^{\circ} \mathrm{y}\right)$ is attained in the fuel bundie, the package is rejocted and no furtner calculations are nade. If the temperature is within limit. tne dackage is then 
INDIVIDUAL BARRIER

\section{BARRIER LAYER}

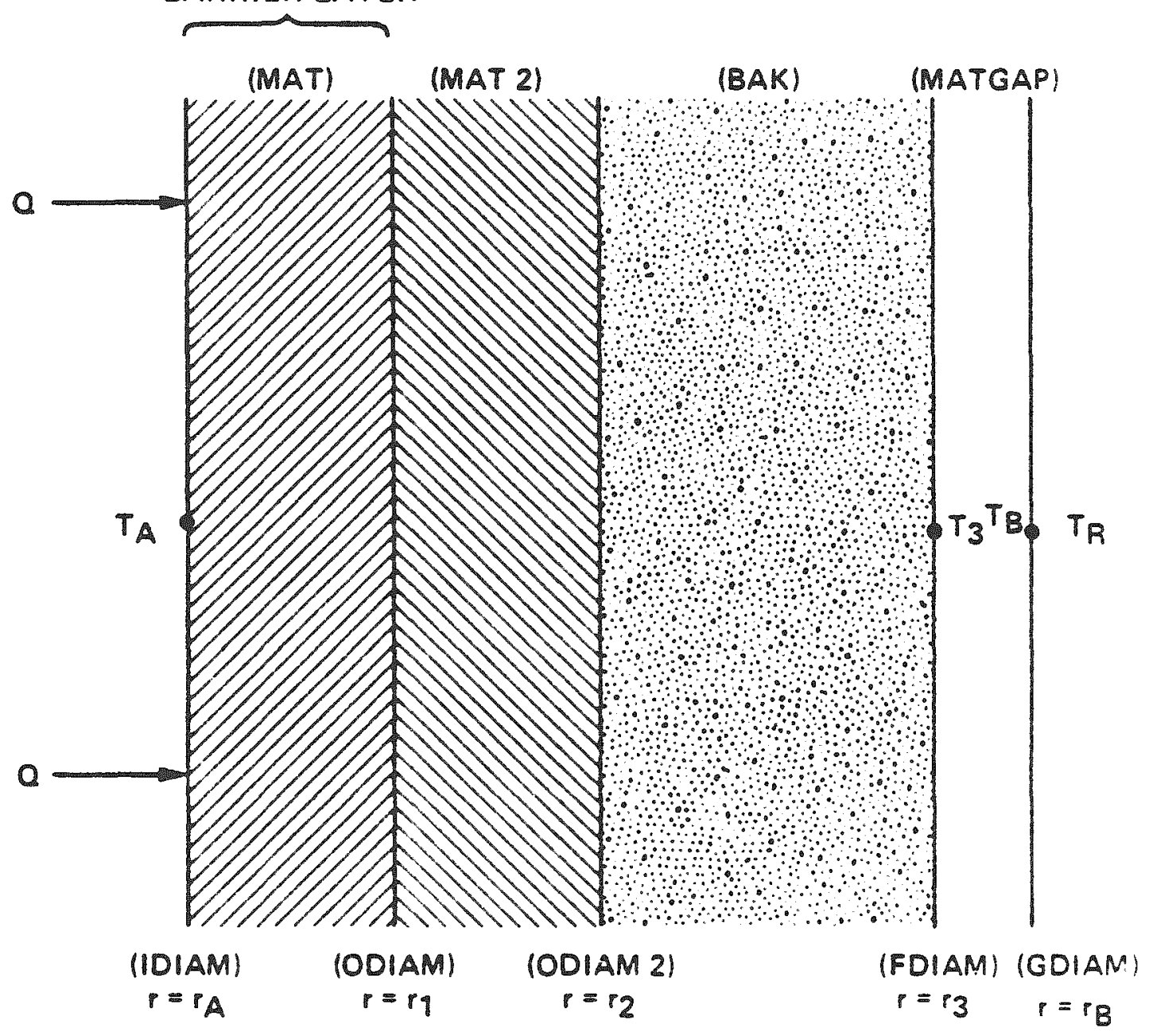

$Q=$ RADIAL WASTE HEAT GENERATION, WATTS

$T_{A}=$ TEMPERATURE AT INSIDE OF LEFTMOST SOLID WALL IOR WASTEI, OK

$T_{B}=$ TEMPERATURE AT OUTSIDE OF RIGHTMOST SOLID WALL (OR REPOSITORY). OK

WHERE

$T_{3}=$ TEMPERATURE AT OUTSIDE OF BACKFILL (OR FILLER), OK

$r=$ RADIUS RELATIVE TO WASTE CENTERLINE $(r=0)$, IN.

$1 \mid=$ DIMENSIONAL OR MATERIAL VARIABLE NAMES USED IN CODE

$T_{R}=$ TEMPERATURE OF REPOSITORY, OK

Figure 3-1. PKTEMP Barrier Mode1. 


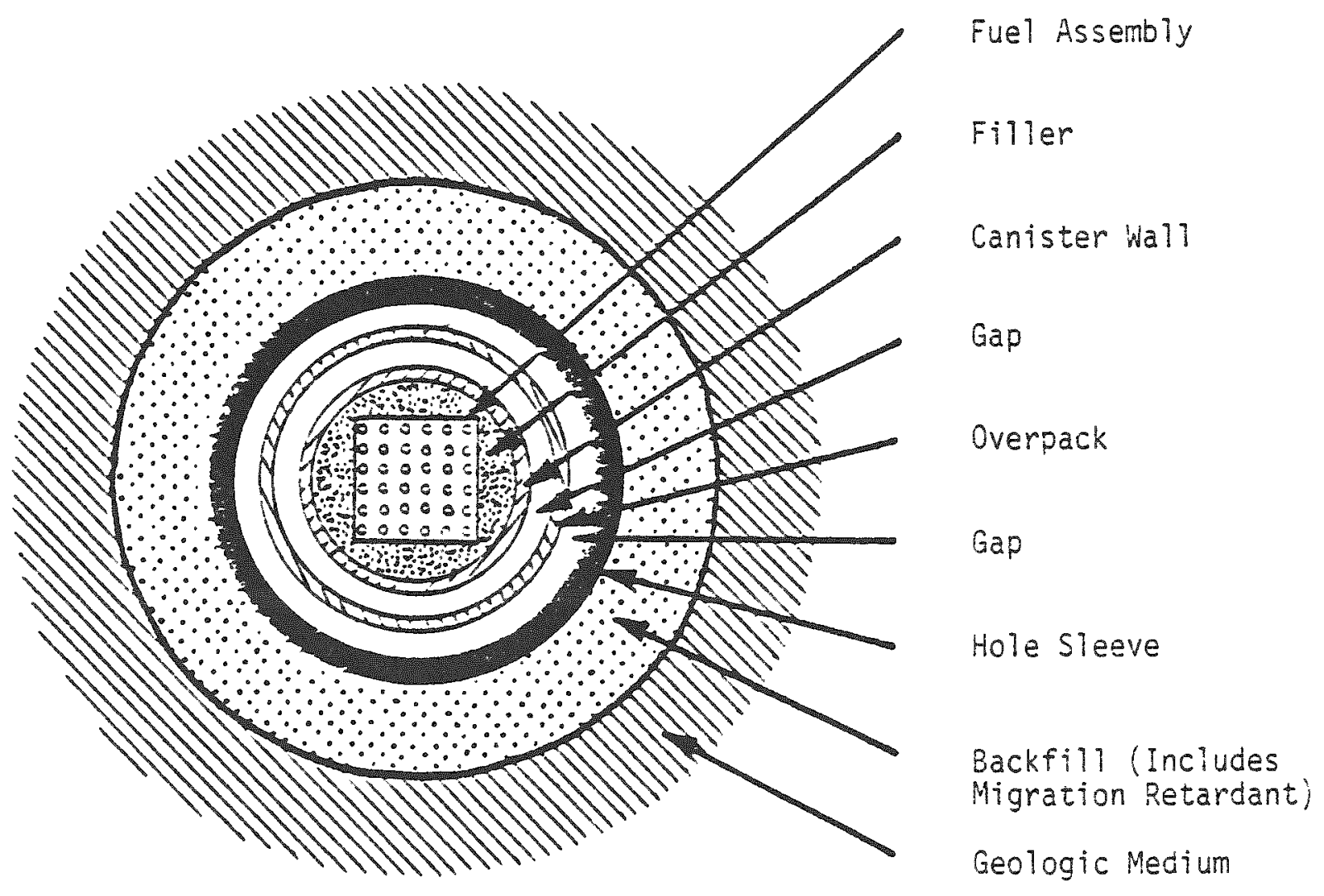

Figure 3-2. Stylized Waste Package Configuration for Fuel Assembly Waste Form. 


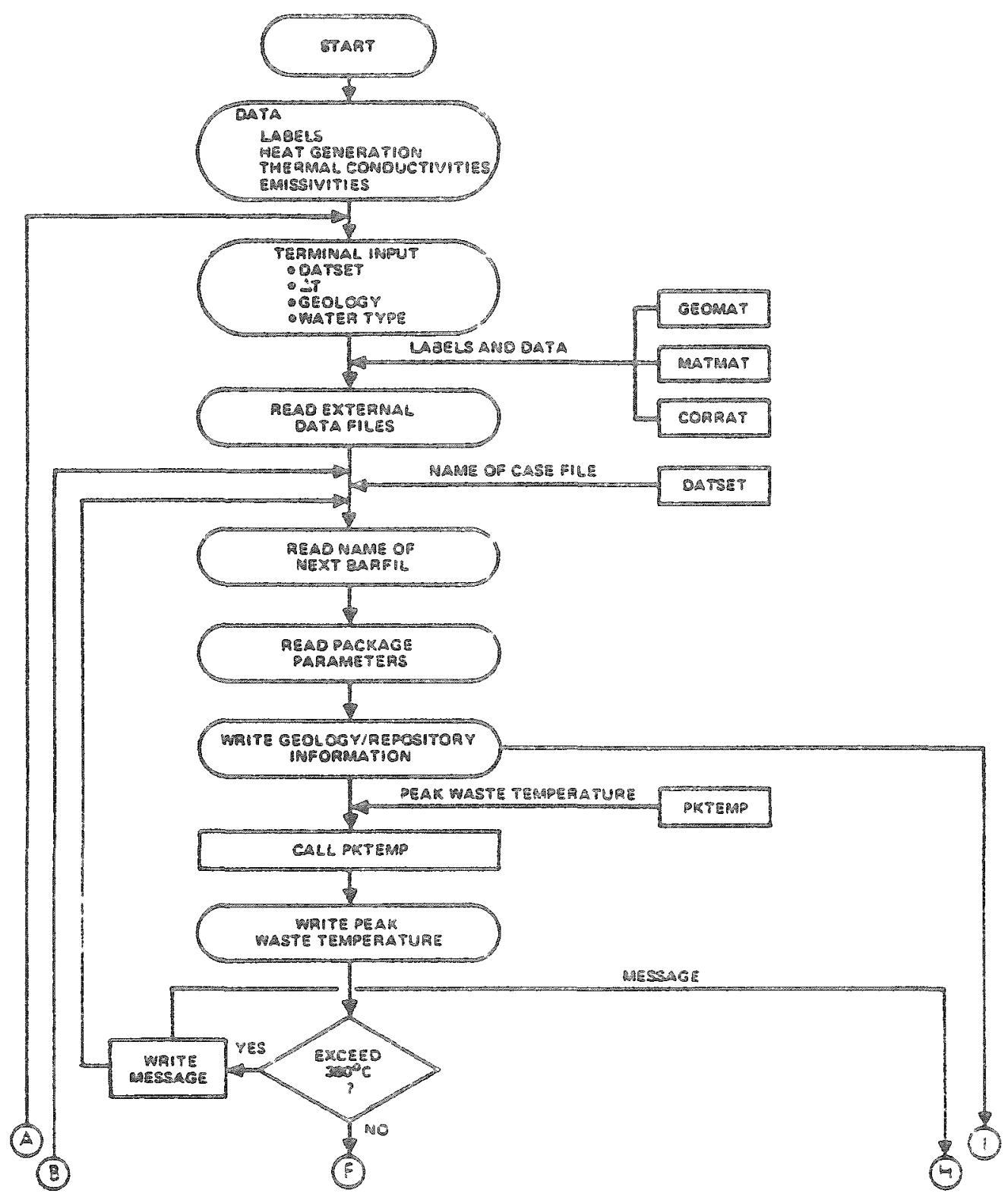

Figure 3-3. Basies Flowchart. 


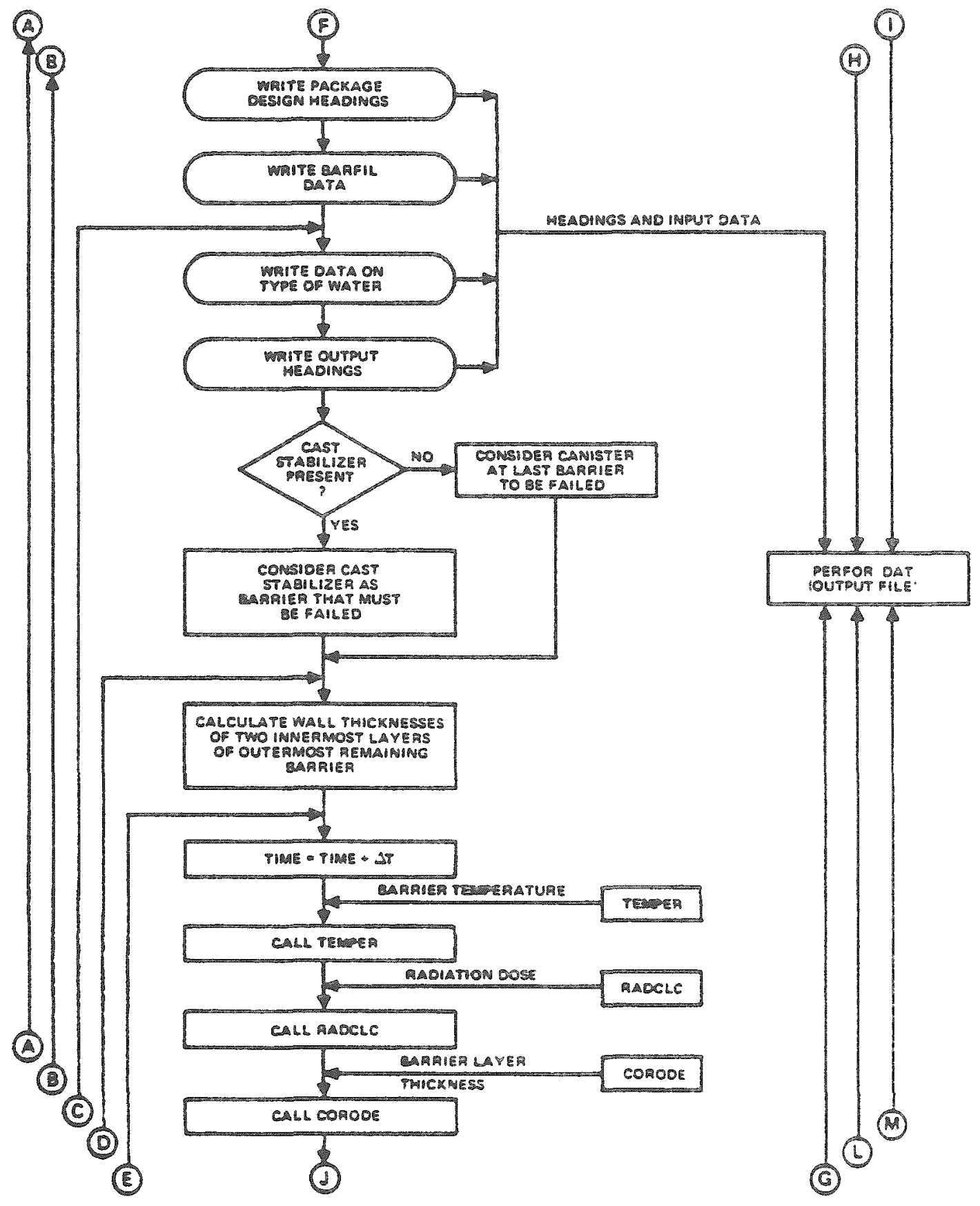

Figure 3-3. BARIER Flowchart Continued, 


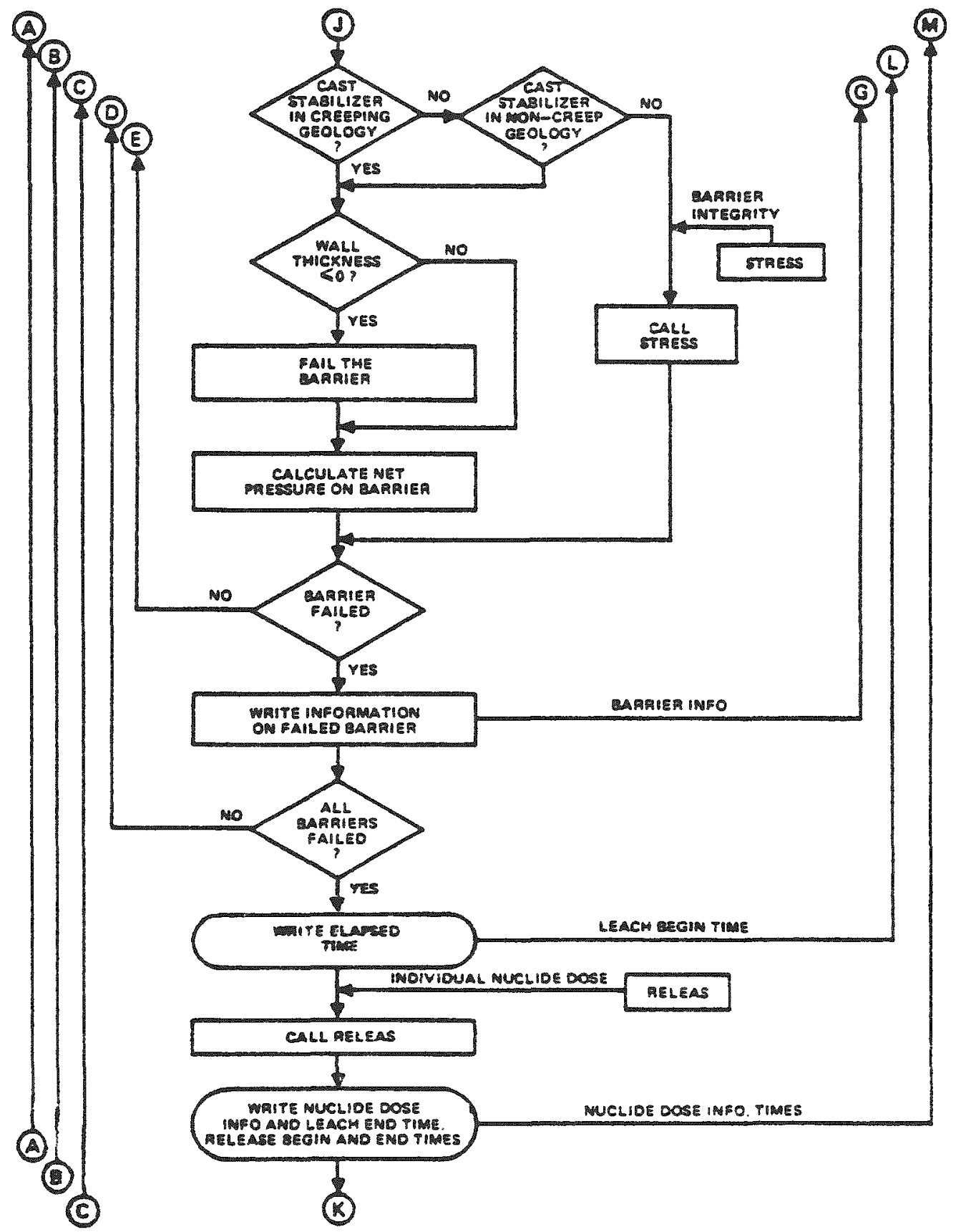

Figure 3-3. BRRIER Flowchart (Continued). 


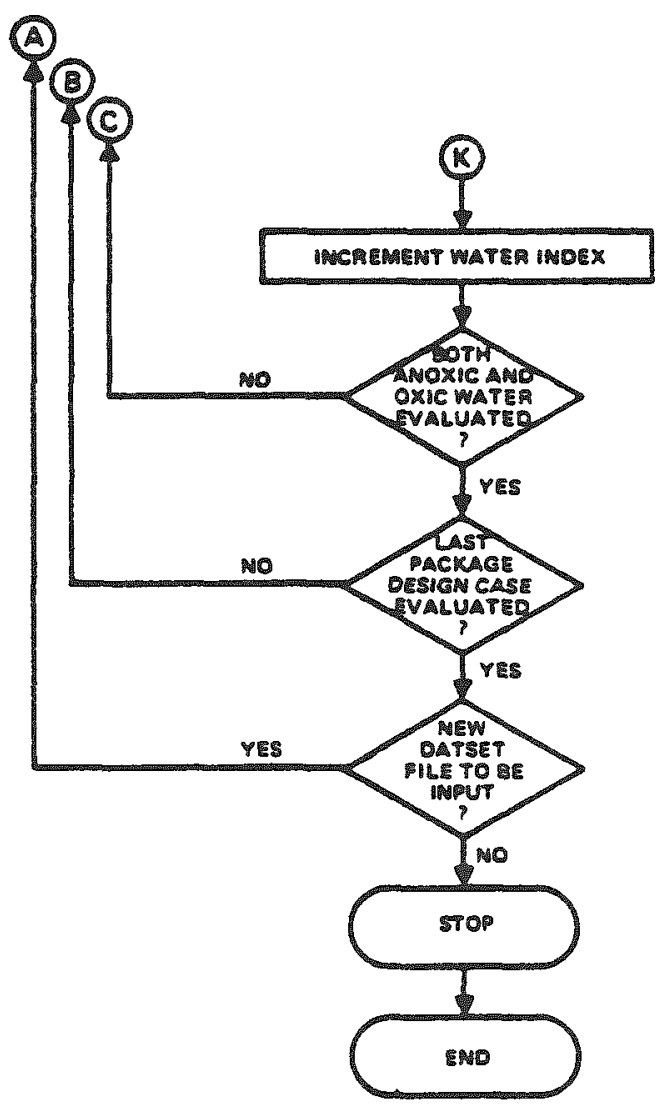

Figure 3-3. BARIER Flowchart (Continued). 
taken through time increments as shown in Figure 3-3. The temperature of the outer barrier is assessad in the heat transfer model and the nuclear radiation field is evaluated. Then the corrosion model determines the decrease in barrier wall thickness for that time increment based on the water chemistry, type of material, and temperature range. The revised wall thickness is checked in a mechanical stress model which calculates displacement and stresses and checks the results against failure criteria. If the element does not fail then time is incremented and the process is repeated. once the barrier fails the next innermost barrier is taken through the process until the last barrier fails. Failure of the last barrier passes control to the waste package release model which includes leaching and transport calculations for specific radionuclides.

Jetails of the specific nodels for each of the subroutines identified above are discussed in the following subsections. Specific package designs evaluated are discussed in Section 4.0 .

3.2 TEMPERATURE CALCILATIONS

There are three temperature calculations perfomed by BARIER and its subroutines:

(1) repository surface temperature as a function of time is calculated in the main program

(2) peak waste temperature is calculated in the PKTEMP subroutine

(3) barrier temperature at time of failure is calculated in the TEAPER subroutine

Table 3-1 details the areal neat loadings assumed for the reference waste repository as described in the GEIS (DOE, April 1979). Temperature calculations perfomed in the GEIS studies are used in the BARIER code. It is conservatively assumed that the bulk temperatures are unchanged by the presence of water from the flooding scenario. An approximate fit to the time-tenserature curves in the aEIS is made for each of the four geologic nedia considered. The repository surface temperature is represented in BARIER by

$$
\begin{array}{ll}
T_{R}=T 1+T 2 \ln t & t \leq T 3 \\
T_{R}=T 4 & t>T 3
\end{array}
$$


Table 3-1. Thermal Loadings Achieved for the Conceptual Repositories for Once-Through Fuel Cycle.

\begin{tabular}{|c|c|c|c|c|}
\hline iremal Loading at Emolacement & Sai: & Sranite & Shale & Basa?: \\
\hline \multicolumn{5}{|l|}{ PNR } \\
\hline$x \sin / \operatorname{can}$ & 0.72 & 0.72 & 0.72 & 0.72 \\
\hline lear field local kw/acre & 50 & 130 & 80 & 120 \\
\hline Far field average kd/acre & 40 & 160 & 55 & $: 00$ \\
\hline \multicolumn{5}{|l|}{ EंiR } \\
\hline$x+\operatorname{sen}$ & 0.22 & 0.22 & 0.22 & 0.22 \\
\hline vear field local kin/acre & 50 & 130 & 55 & $i 30$ \\
\hline Far field average $\mathrm{kw} / \mathrm{acre}$ & 40 & 100 & $\$ 4$ & 100 \\
\hline
\end{tabular}


where

$$
\begin{aligned}
T 1, T 2, T 3, T 4 & =\text { constants for the fit } \\
t & =\text { time after emplacement, }(y r)
\end{aligned}
$$

The fit is further conservative in that the temperature is assumed constant beyond time $T 4$ when there is actually a gradual decrease in temperature. Values of constants used are presented in Table $3-2$.

The PKTEMP subroutine calculates the peak waste temperature expected during the life of the waste package. A concentric cylinder nodel is used which accounts for heat transfer by conduction and radiation. Exploratory calculations revealed that free convection effects are small and that coefficients tend to approach pure conduction. When the waste package has gaps between barrier elements, heat radiation effects are significant and are included in the model.

The peak waste temperature is determined by calculating the temperature differential across a series of individual barrier heat transfer resistances while utilizing the maximum repository temperature as the reference temperature. An infinite-length concentric cylinder heat transfer equation is used which assumes individual barrier resistances as depicted in Figure 3-1. Each barrier resistance is modeled as having a maximum of four distinct layers across which heat transfer occurs. These include an inner solid wall, an outer solid wall (e.g., a corrosion-resistant cladding), a filler or backfill material, and a zao between barriers. The variable names corresponding to the inner dianeter and material of each of these layers are shown in parentheses in Figure 3-1. These variables are generally subscripted with the variable I to distinguish between individual barriers $(I=I$ for the innermost barrier and $I=13$ for the outermost barrier).

Heat transfer across the first three layers of each barrier $\left(r_{7}\right.$ to $\left.r_{3}\right)$ is assumed to occur by conduction only. The following conduction equation is used in the code

$$
Q / L=\frac{2 \pi\left(T_{A}-T_{3}\right)}{\frac{2 n\left(r_{1} / r_{A}\right)}{k^{A I}}+\frac{2 n\left(r_{2} / r_{1}\right)}{k^{12}}+\frac{2 n\left(r_{3} / r_{2}\right)}{k^{23}}}
$$


Table 3-2. Constants For Repository Temperature Calculations.

\begin{tabular}{|l|c|c|c|c|}
\hline Geology & $\mathrm{T} 1,\left({ }^{\circ} \mathrm{C}\right)$ & $\mathrm{T} 2,\left({ }^{\circ} \mathrm{C} / \mathrm{yr}\right)$ & $\mathrm{T} 3,(\mathrm{yr})$ & $\mathrm{T} 4,\left({ }^{\circ} \mathrm{C}\right)$ \\
\hline SALT & 122.66 & 23.60 & 20.00 & 193.00 \\
BASALT & 128.80 & 31.15 & 10.00 & 200.00 \\
GRANITE & 129.24 & 29.97 & 10.00 & 198.00 \\
& 100.45 & 30.00 & 15.00 & 182.00 \\
\hline
\end{tabular}


where

= waste heat generation, (Natts)
$k^{A 1}, k^{12}, k^{23}=$ thermal conductivity of layer, (Watts/in $-{ }^{0} k$ )
L $\quad=$ length of waste heat generation surface, (in)

Values of thermal conductivity are assumed constant and taken at the midpoint of the temperature range considered. Heat transfer across the gap $\left(r_{3}\right.$ to $\left.r_{3}\right)$, if present, is assumed to occur by both conduction and radiation. The following equations are used for this situation.

$$
\begin{gathered}
Q / L=2 \pi\left[r_{3} \sigma e\left(T_{3}^{4}-T_{B}^{4}\right)+\frac{T_{3}-T_{B}}{\frac{\ln \left(r_{B} / r_{3}\right)}{k^{3 B}}}\right] \\
\varepsilon=\frac{1}{\frac{1}{e_{3}}+\frac{r_{3}}{r_{B}}\left(\frac{1}{e_{B}}-1\right)}
\end{gathered}
$$

where

$$
\begin{aligned}
& \sigma=3.68 \times 10^{-11},\left(\text { watts } / i^{2}-{ }^{0} k^{4}\right) \\
& e=\text { effective emissivity, (dimensionless) } \\
& e_{3}=\text { emissivity at surface } 3, \text { (dimensionless) } \\
& e_{B}=\text { emissivity at surface } 3, \text { (dimensionless) } \\
& k^{3 B}=\text { thermal conductivity across gap, ('Natts/in- }{ }^{0} k \text { ) }
\end{aligned}
$$

Values of emissivity are assumed constant over the range of temperature considered. 
The PKiEMP calculations are perforned using the logic depicted in the flowchart shcen in Figure 3-4. Initially, the maximum repository temperature is determined. inis is followed by determination of the temperature across each barrier st.rting with the outermost barrier and proceeding inward. For each barrier, the code first determines if a gap is present as defined in the nodel shown in figure 3-1. If no gap is present, the code skips over the radiation/conduction heat transfer equation and sets $T_{3}=T_{B}$. The conduction equation calculates $T_{A}$ for the barrier and sets $T_{B}$ of the next innermost barrier equal to $T_{A}$. This process is repeated until the innemost barrier is reached. If a gap is present in any of the barriers, the code tests for the presence of a filler (backfill) material in that barrier and chooses the appropriate emissivities for use in the radiation/conduction heat transfer equation. A variable $P=f\left(T_{3}, T_{B}\right)$ is evaluated in an iterative technique to soive for $T_{3}$

$$
P=2 \pi\left[3.68 \times 10^{-11} r_{3} e\left(T_{3}^{4}-T_{B}^{4}\right)+\frac{T_{3}-T_{B}}{\frac{2\left(r_{8} / r_{3}\right)}{k^{3 B}}}\right]-Q / L
$$

An initial $T_{3}$ is assumed equal to $T_{B}$ and $P$ is calculated. $T_{3}$ is then succesively increnented until $P$ converges giving the desired value of $T_{3}$. $T_{A}$ for the barrier is then solved by the conduction equation. $T_{A}$ for the innermost barrier is assumed to be the peak waste temperature (MAXTMP) for the waste package. $A$ program listing of PKTEMP is provided in Appendix A.

The TEMPER subroutine caiculates the temperature of a barrier at tne time of barrier failure. Barrier failure is defined as a breakthrough of the innermost layer (solid wall) of a barrier. TEMPER performs a neat transfer calculation between the repository surface and the outer barrier surface utilizing a calculated repository temperature and an estinated overall neat transfer coefficient. TEMPER calculations are performed using the logic depicted in the flowchart shown in Figure 3-5.

The program first calculates the repository temperature as a function of tine. The outermost barrier temperature at failure is then calculated using the following equation 


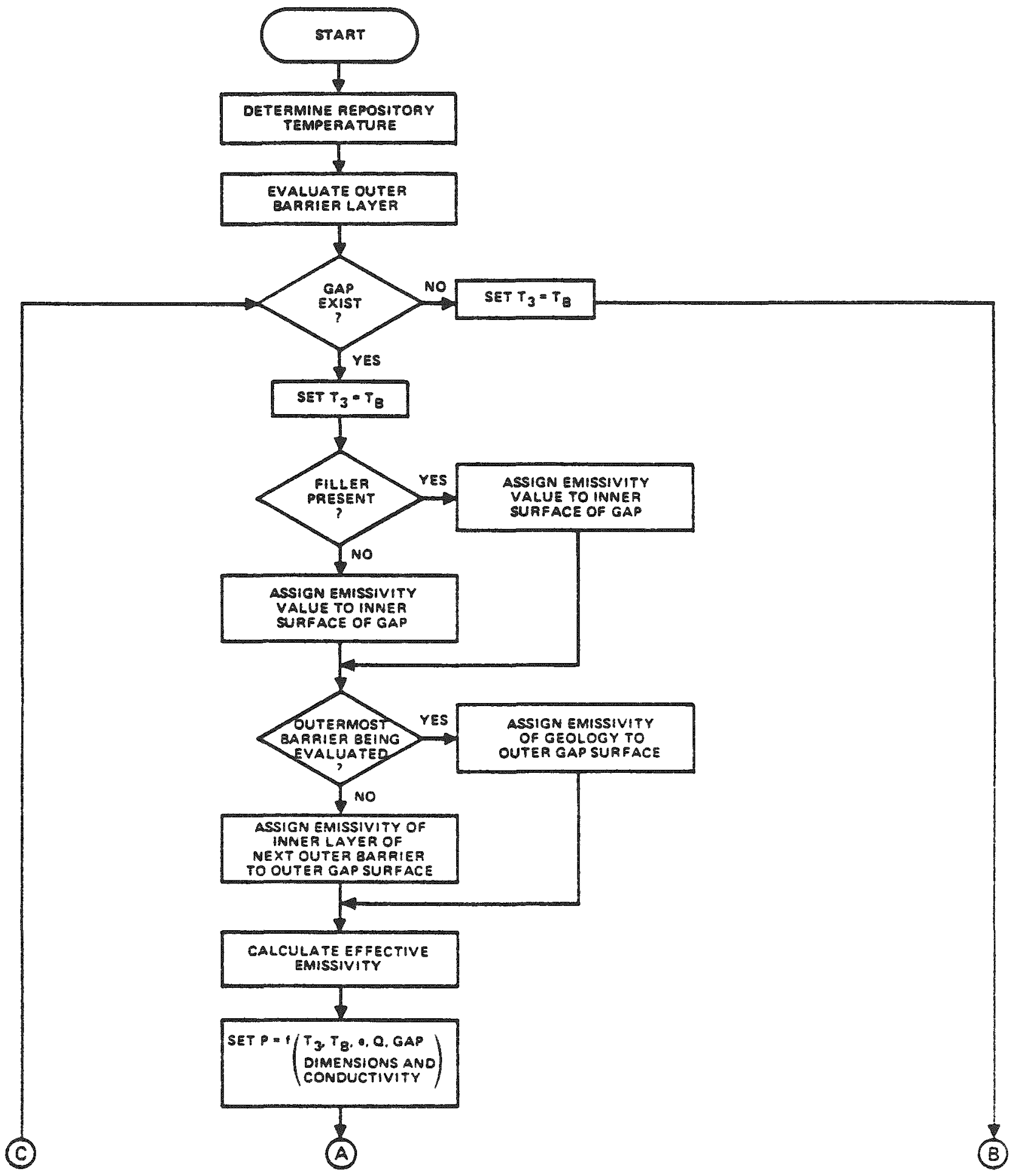

Figure 3-4. PKTEMP Flowchar:. 
(C)

(A)

(B)

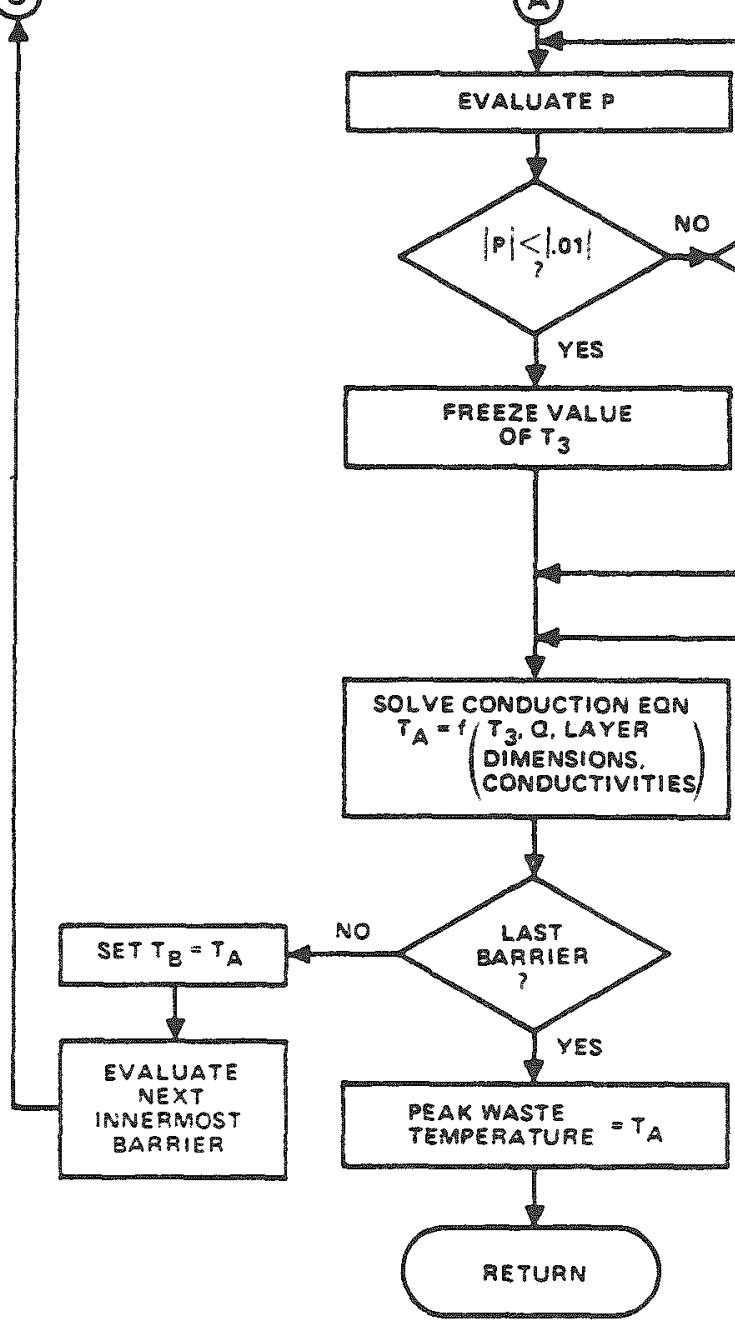

Figure 3-4. PKTEMP Flowchar: (Continued). 


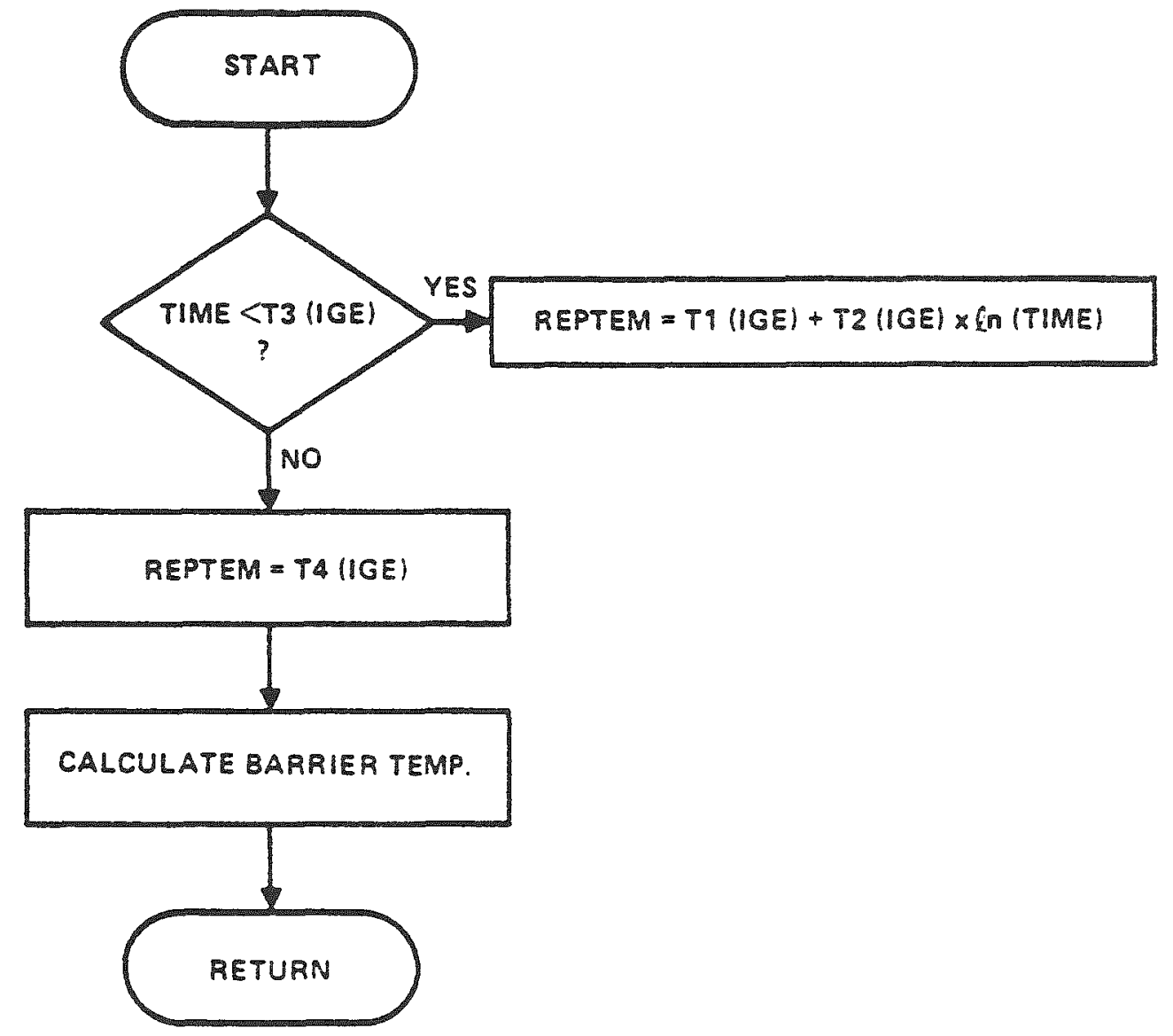

Figure 3-5. TEMPER Flowchart. 


$$
T_{C}=\frac{Q / L}{2 \pi h_{B R}\left(r_{A}+2 \Delta r\right)}+T_{R}
$$

where

$T_{c}=$ barrier temperature at outer surface, $\left({ }^{\circ} k\right)$ $\begin{aligned} h_{B R}= & \text { estimated overall heat transfer coefficiant between } \\ & \text { repository and barrier surface, (Watt/in } n^{2}-K \text { ) }\end{aligned}$

$T_{R}=$ calculated repository temperature, $\left(O_{K}\right)$

$\Delta r=$ thickness of inner barrier layer at time of failure, (in)

For each successive barrier, the repository temperature of the particular geology in question is recalculated and is dependent only upon time. The accuracy of the heat transfer coefficient estimate is relatively unimportant in that typical waste heat generation is such that in the designs studied, calculated barrier temperatures at failure are nearly equal to the repository temperature. A listing of the TEMPER subroutine is provided in Appendix A.

\subsection{CORROSION}

The CORODE subroutine calculates the thicknesses of the two inner layers (Figure 3-1) of each barrier as a function of time. In each case a corrosion rate is chosen on the basis of temperature and type of repository water and is utilized to calculate the decreasing thickness of a solid barrier wall. The model assumes that the corrosion rate is characteristic of full immersion conditions. The general form of the corrosion equation is as follows

$$
\begin{aligned}
& \qquad x_{1}=x_{0}-R_{c} \Delta t \\
& x_{1}=\text { new thickness, }(i n) \\
& x_{0}=\text { previous thickness, }(i n) \\
& z_{c}=\text { corrosion rate, }(\mathrm{in} / \mathrm{yr}) \\
& \dot{\text { it }}=\text { time increment, }(\mathrm{yr})
\end{aligned}
$$


The CORODE calculations are perfomed using the logic depicted in the flowchart shown in figure 3-6. The program first tests for the existence of a corrosion-resistant coating on the outside of the outermost of the two barrier layers in question (Figure 3-1). If present, the coating is defined in terms of time of protection afforded to the surface to be corroded. This length of time is specified in the specific input data files such that the CORODE corrosion calculations do not begin until the specified time period has elapsed. Once coprosion is ready to begin, the program determines the temperature and existing repository water type before choosing the appropriate corrosion rate from the data file CORRAT. For each pass through CORODE, the outer of the two layers in question is decreased in thickness by an amount equal to the corrosion rate tines a time increment (specified in input to main program BARIER). Successive calculations occur until terminated by zero cladding layer thickness. After failure of the outer layer, the inner layer is corroded using the appropriate corrosion rate until it fails by either zero thickness of excessive external stress. Once a complete barrier fails, the next innermost barrier is considered to be uniformly flooded and the entire process is repeated. In the event of a barrier with no solid walls to be corroded (e.g., air or helium stabilizer), the two innermost barrier layers are considered to be zero and CORODE is not utilized.

The corrosion rate data contained in CORRAT is comprised of eignt separate values for each package material (metals). Four corrosive environments are considered

\section{(1) Anoxic brine $B$ \\ (2) Oxic brine $B$ \\ (3) Anoxic water \\ (4) Oxic water}

over two temperature ranges $\left(25^{\circ}-100^{\circ} \mathrm{C}, 100^{\circ}-250^{\circ} \mathrm{C}\right)$. The chenical compositions of brine $B$ and typical groundwater are summarized in Taoles $3-3$ and $3-4$, respectively. Each corrosion rate is assumed constant over its temeerature range and is taken from the maximum of rates corresponding to specific corrosion 


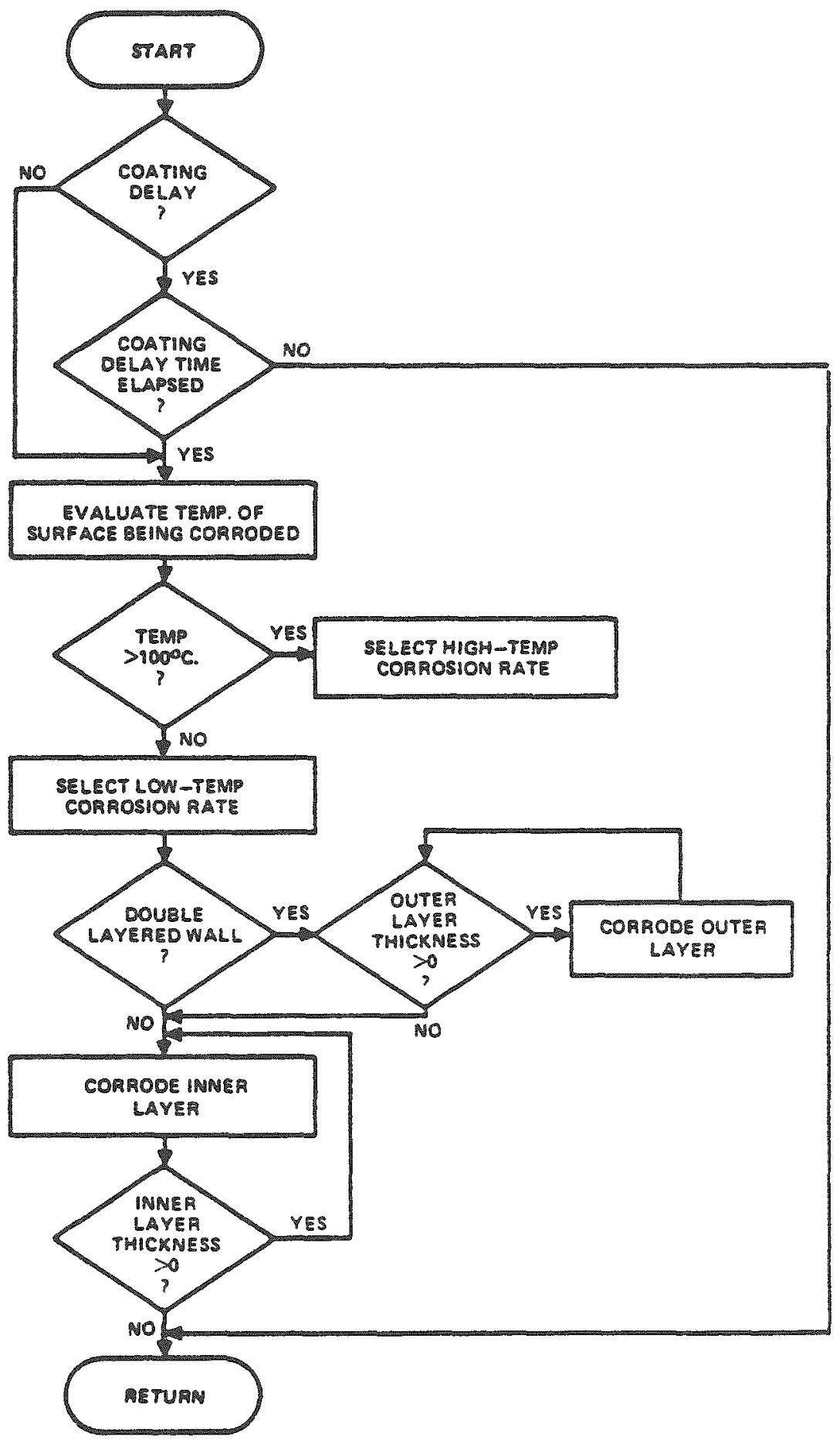

Figure 3-6. COROOE Flowenart. 
Tabie 3-3. Chemical Compostion of WIPP-B Salt Brine*

\begin{tabular}{|l|c|}
\hline \multicolumn{1}{|c|}{ Compound } & Concentration $(\mathrm{g} / \mathrm{2})$ \\
\hline $\mathrm{NaCl}$ & 287.00 \\
$\mathrm{Na}_{2} \mathrm{SO}_{4}$ & 6.20 \\
$\mathrm{Na}_{2} \mathrm{~B}_{4} \mathrm{O}_{7} \cdot 1 \mathrm{H}_{2} \mathrm{O}$ & 0.0160 \\
$\mathrm{NaHCO}_{3}$ & 0.0140 \\
$\mathrm{NaBr}$ & 0.5200 \\
$\mathrm{KCl}$ & 0.0290 \\
$\mathrm{KI}$ & 0.0130 \\
$\mathrm{MgCl}$ & 0.0400 \\
$\mathrm{CaCl}_{2} \cdot 2 \mathrm{H}_{2} \mathrm{O}$ & 3.30 \\
$\mathrm{FeCl}_{3}$ & 0.0060 \\
$\mathrm{SrCl}_{2} \cdot 2 \mathrm{H}_{2} \mathrm{O}$ & 0.0330 \\
$\mathrm{Rb}_{2} \mathrm{SO}_{4}$ & 0.0016 \\
$\mathrm{CsCl}$ & 0.0013 \\
$\mathrm{Total}^{\mathrm{Dissolved}}$ & $297.174 \mathrm{~g} / \mathrm{i}$ \\
$\mathrm{PH}$ & 6.5 \\
\hline
\end{tabular}

*Braithwaite and Molecke, 1979 
Table 3-4. Chemical Composition of Typical Arid Ground Water*.

\begin{tabular}{|l|ll|}
\hline Compound & \multicolumn{2}{|c|}{ Concentration $(\mathrm{mg} / \mathrm{l})$} \\
\hline Sulfate & $<50$ & $(1-20)$ \\
Chloride & $<100$ & $(2-50)$ \\
Bicarbonate & $<500$ & $(60-400)$ \\
Nitrate & $<10$ & $(0.1-5)$ \\
Sodium & $<50$ & $(5-47)$ \\
Potassium & $<10$ & $(1-5)$ \\
Magnesium & $<50$ & $(2-20)$ \\
pH & $7-9$ & $(6.8-8.5)$ \\
\hline
\end{tabular}

*Katayama, 1976 
mechanisms for a particular environment and temperature range. The corrosion mechanisms considered included uniform corrosion, stress corrosion, pitting, and graphitization (see Appendix B). The general form of CORRAT is shown in Table $3-5$ and a listing of CORRAT with current data is provided in Appendix E. A program listing of CORODE is provided in Appendix A. Package materials for which corrosion data are obtained include mild steel, Zircaloy-2, Inconel-600, 304 Stainless Steel, copper, lead, and cast iron.

A degree of uncertainty in the corrosion rate data base exists because of the numerous effects of environmental parameters on package corrosion. Environmental parameters acting upon waste packages vary with the geology of the repository and can have a major impact on resultant corrosion rates. For example, increases in temperature generally increase the corrosion rates of metals (Braithwaite, 1979). Also, increases in temperature in an open system cause a depletion in dissolved oxygen in aqueous solutions. This decreases the corrosion rate of metals whose rate is controlled by diffusion of oxygen.

The restraining pressure which a waste package is subjected to in a repository affects the corrosion rate primarily in that it influences the physical state of intruding water and the concentration of dissolved zaseous species. Waste packages will be exposed to any thermal decomposition products of the geologic isolation formation and any dissolved and gaseous species present. In general, species in solution which increase the oxidizing power of that solution increase the corrosion rate.

The tensile stress present in the barrier wall is one of the essential requirements for stress corrosion cracking. Not all naterials are susceptible to stress corrosion cracking in geologic isolation conditions. For susceptible materials, the threshold tensile stress depends strongly on temperature, solution composition, and the presence of an aqueous phase. Alloys containing carbon and chromium can be susceptible to sensitization. For example, sensitization in stainless steels refers to the thermally induced formation of chromium carbide at or near grain boundaries (Molecke, 1979). This increases the susceptibility of the alloy to intergranular attack and intergranular stress corrosion cracking. idelding, because of the high temperatures involved, often leads to sensitization and tensile stress in welded regions.

The corrosion rate data base is generally considered to be conservative in view of the procedure used to choose maximum corrosion rates for each set of temperature and water conditions. In addition, potential effects on corrosion 
Table 3-5. General Form of CORRAT.

\begin{tabular}{|c|c|c|c|c|c|c|c|c|}
\hline \multirow{2}{*}{ MatrRIML } & \multicolumn{2}{|c|}{ BRIME-AMOXIC } & \multicolumn{2}{|c|}{ BUINE-OXIC } & \multicolumn{2}{|c|}{ WAIER-AMOXIC } & \multicolumn{2}{|c|}{ WAIER-OXIC } \\
\hline & $25^{\circ}-100^{\circ} \mathrm{C}$ & $100^{\circ}-250^{\circ} \mathrm{C}$ & $25^{\circ}-100 \cdot C$ & $100^{\circ}-250^{\circ} \mathrm{C}$ & $25^{\circ}-100^{\circ} \mathrm{C}$ & $100^{\circ}-250^{\circ} \mathrm{C}$ & $25^{\circ}-100^{\circ} \mathrm{C}$ & $100^{\circ}-150^{\circ} \mathrm{C}$ \\
\hline \multicolumn{9}{|l|}{ Muld Stcel } \\
\hline \multicolumn{9}{|l|}{ Zurcalay } \\
\hline \multicolumn{9}{|l|}{ Incunel } \\
\hline \multicolumn{9}{|l|}{304 SSI } \\
\hline \multicolumn{9}{|l|}{ Copper } \\
\hline Lead & & & & & & & & \\
\hline Cast Iron & & & & & & & & \\
\hline
\end{tabular}


rates caused by radiation levels within the waste package only service to reinforce the use of a conservative "worst-case" approach in performing the corrosion calculations.

\subsection{BARRIER FAILURE CRITERIA}

The STRESS subroutine determines the time when a particular package barrier fails due to internal or external pressure. Failure of any metal barrier wall due to external pressure is considered to occur when the wall is in plastic strain and there is a uniform pressure across the wall ("hydrostatic"). Failure due to internal pressure is defined as the time when the wall thickness no longer meets the requirements for hoop stress in the AMSE code, Section VIII, Division 1. The wall thickness is that portion of the original wall not affected by corrosion (including bulk corrosion, pitting, or crack propagation) as determined in the corrosion subroutine. The subroutine updates two binary flags BFAIL and WFAIL. If BFAIL = 0 then the backfill has "failed", which means there is no longer a pressure gradient across the backfill. If WFAIL $=0$, then the solid wall has failed. If WFAIL or BFAIL $=1$, then they are intact, sustaining a pressure gradient.

In each time increment the wall thickness and temperature of a barrier are revised. Then the STRESS subroutine recalculates the new stress distribution and updates the binary flags BFAIL and WFAIL. The main program acts on the value of WFAIL to determine when the defense shifts to the next inner package barrier. BFAIL is used by STRESS to determine the nature of the pressure distribution.

The barrier is considered as a bimetallic wall adjacent to a porous filler (or backfill as depicted in figure 3-7. The model is based on assumption of a structural wall, a cladding with no strength attributes, and a structural backfill. Stress-strain properties of the backfill and structural wall determine pressure profiles between $R_{0}$ and $R_{1}$ and $R_{2}$ and $R_{3}$. The pressure is assumed uniform between $R_{1}$ and $R_{2}$.

The mechanical properties of backfill materials vary widely depending on the minerals, particle size and shape distributions, porosity, and moisture content. It is assumed that the backfill is loaded monotonically by creep of the geologic media as it acts to close the borehole.

The yield model used for the backfill is

$$
Y=C_{0}+B\left(P_{R}-P_{\text {pore }}\right)
$$




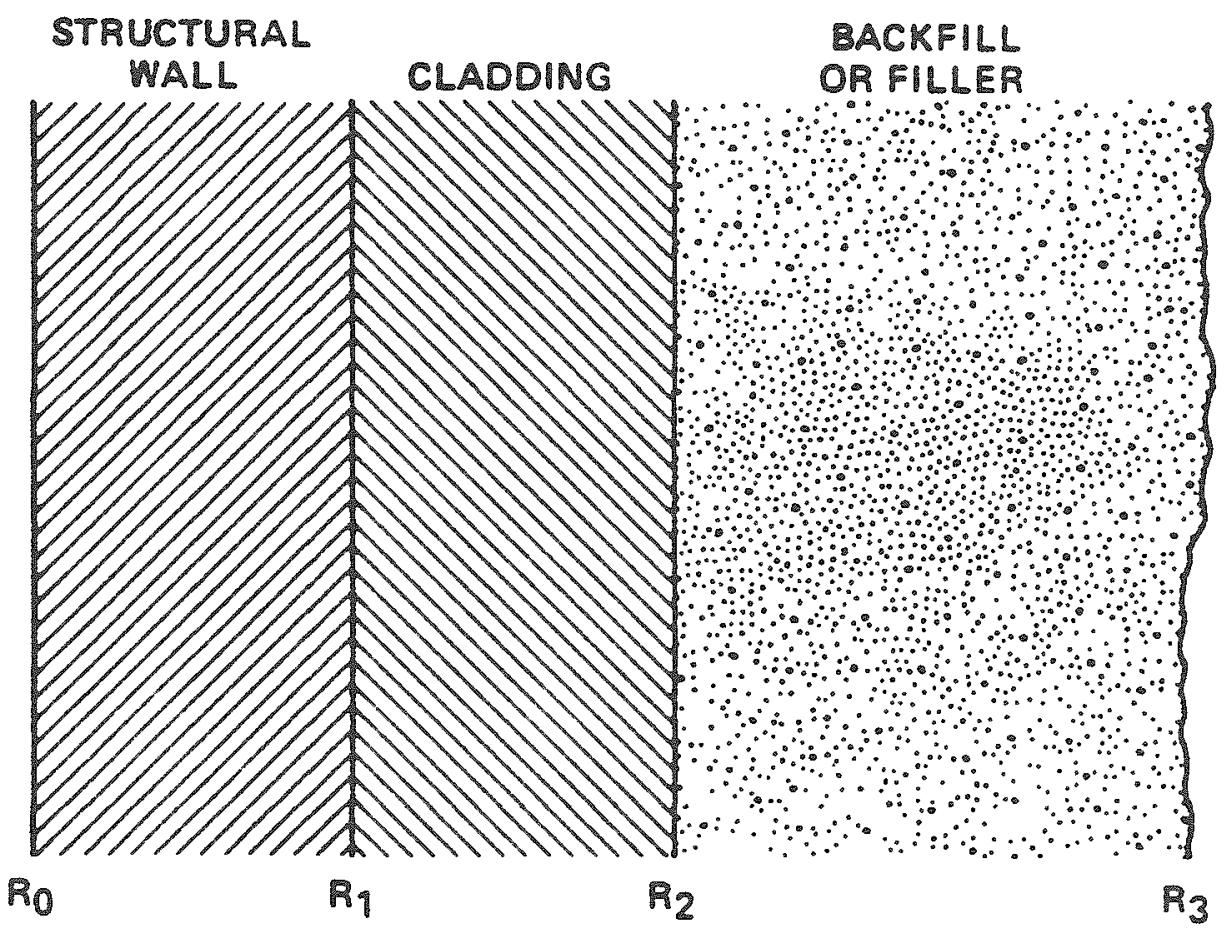

Figure 3-7. Composite Barrier Used in Stress Calculations. 
where

$$
\begin{aligned}
& C_{0} \quad=\text { cohesion, }(K s i) \\
& 3 \quad=\text { constant slope of the Mohr envelope, (dimensionless) } \\
& P_{R} \quad=\text { absolute value of the mean stress (repository pressure), (Ksi) } \\
& P_{p o r e}=\text { pore water pressure, }(K s i)
\end{aligned}
$$

In this application cohesion is neglected. This could be added later if necessary but is reasonable for most materials in question. For most compacted soils the Mohr slope falls becween 0.6 and 1.2. $P_{\text {pore }}=0$ for dry materials and ranges up to about $P / 2$ based on a ratio of the weight of a water column from the repository to surface and the weight of the overburden of typical rock. In the subroutine the yield stress is

$$
Y=S\left(P_{R}-P_{\text {pore }}\right)
$$

Generally, the conservative assumption of $P_{\text {pore }}=0$ is used. Note that this model assumes also that creep is sufficiently rapid that overburden pressure is applied at time $=0$.

The pressure-volume relationship for the backfill is a very non-linear and highly variable-based physical characteristic. Any backfill employed must have defined an empirical pressure-volume characteristic. To accomnodate the wide range of possibilities, a quadratic data fit is provided in the model using two coefficients. A relation between volume before and after compression is

where

$$
V^{*}=\frac{V_{0}}{V}-1
$$

$$
\begin{aligned}
& V_{0}=\text { original volume, }\left(\mathrm{cm}^{3}\right) \\
& V=\text { volume after compression by pressure } 2,\left(\mathrm{~cm}^{3}\right)
\end{aligned}
$$


and

$$
P_{R}=A V^{*}+K V^{2}
$$

where $A$ and $K$ are empirical coefficients. Thus,

$$
V^{*}=\frac{-A \pm \sqrt{A^{2}+4 K P_{R}}}{2 K}
$$

In the special case where $k=0$ or $k \approx 0$

$$
V^{*}=\frac{P_{R}}{A}
$$

Equation (3.4.5) refers to a "soft" backfill which displays a high degree of volume reduction under small compressiveloads. Equation (3.4.6) refers to a "stiff" backfill with low compaction at high pressure. Typical data (3yerlee, $1967)$ gives a curve for compacted sand

$$
P_{R}=0.4413 V *+253 V *^{2}
$$

The instantaneous shear modulus, $G^{\prime}$, was constructed from the instantaneous bulk modulus, $B^{\prime}$, and Poisson's ratio, $\nu$, according to non-linear elastic theory. The bulk modulus is given by

$$
B^{-}=-V \frac{d P_{R}}{d V^{\prime}}
$$

and snear modulus by

$$
G^{-}=\frac{3}{2} B^{-} \frac{(1-2 v)}{(1+v)}
$$

The Lame' constant is given by

$$
A^{\circ}=B^{-}-\frac{2 G^{\circ}}{3}
$$


Differentiating Equation $(3.4 .4)$ and substituting into Equation $(3.4 .3)$ gives

$$
B^{\prime}=A\left(Y^{*}+1\right)+2 K V^{*}\left(V^{*}+1\right)
$$

Then $G^{\prime}$ and $\lambda^{\prime}$ are calculated by Equations (3.4.9) and (3.4.10).

The barrier wall is assumed to be constructed of an elastic material for which the following properties are specified as functions of temperature, $T$

$$
\begin{aligned}
& \text { Yield Stress }=Y=Y_{1}+Y_{2}(T) \\
& \text { Bulk Modulus }=B=B_{1}+B_{2}(T) \\
& \text { Shear Modulus }=G=G_{1}+G_{2}(T)
\end{aligned}
$$

The Poisson ratio is calculated by

$$
\nu=\frac{1}{2}\left(\frac{3 B-2 G}{3 B+G}\right)
$$

A stress equilibrium calculation is employed to calculate the response of the cylindrical composite at a stressed state as compared with pressures and dimensions in an unstressed state. Because changes occur very slowly, it is reasonable to assume equilibrium.

Consider a single cylinder of outside radius $R$ and length $z$. In Jeneral the stress and strain changes are related by the elastic equations

$$
\begin{aligned}
& \Delta \sigma_{R}=(\lambda+3 G) \varepsilon_{R}+\lambda \varepsilon_{\theta}+\lambda \varepsilon_{z} \\
& \Delta \sigma_{\theta}=\lambda \varepsilon_{R}+(\lambda+2 G) \varepsilon_{\theta}+\lambda \varepsilon_{z} \\
& \Delta \sigma_{z}=\lambda \varepsilon_{R}+\lambda \varepsilon_{\theta}+(\lambda+2 G) \varepsilon_{z}
\end{aligned}
$$

whers

$$
\sigma_{R}, \sigma_{3}, \sigma_{Z}=\text { stress components, (Ksi) }
$$




$$
\begin{aligned}
\varepsilon_{R}=d U / R & =\text { change of radial strain, (in/in) } \\
U & =\text { strain, (in) } \\
v_{z} & =\text { change of hoop strain, }(i n / i n) \\
\varepsilon_{z} & =\text { change in axial strain, }(i n / i n)
\end{aligned}
$$

Initial equilibrium stress results in no motion

$$
\begin{gathered}
\rho \ddot{R}=\frac{d \sigma^{0}}{d^{R}}+\left(\sigma_{R}^{0}-\sigma_{\theta}^{0}\right) R=0 \\
\rho \ddot{z}=\frac{d \sigma_{z}^{0}}{d z}=0
\end{gathered}
$$

The final equilibrium stresses must produce no motion

$$
\begin{gathered}
\rho \ddot{R}=0=\frac{d}{d R}\left(\sigma_{R}^{0}+\Delta \sigma_{R}\right)+\left(\sigma_{R}^{0}+\Delta \sigma_{R}-\sigma_{\theta}-L \sigma_{\theta}^{0}\right) / R \\
\rho \ddot{z}=0=\frac{d}{d z}\left(\sigma_{z}^{0}+\Delta \sigma_{z}\right)
\end{gathered}
$$

For equilibrium (neglecting rotation)

$$
\begin{gathered}
\rho \ddot{R}=0=\frac{d}{d R}\left(\Delta \sigma_{R}\right)+\left(\Delta \sigma_{R}-\Delta C_{B}\right) / R \\
\rho \ddot{z}=0=\frac{d}{d z}\left(\dot{L} c_{z}\right)
\end{gathered}
$$

The corresponding equation for rotation was left out as it is assumed that there are no torquing forces. Substituting Equations $(3.4 .16)-(3.4 .13)$ into Equations $(3.4 .23)$ and $(3.4 .24)$ and noting that $\varepsilon_{R}=d u / R, \varepsilon_{\theta}=j / R$ and $\frac{d \varepsilon_{z}}{d R}=0$ 
then

$$
(\lambda+2 G) \frac{d^{2} U}{d R^{2}}+\frac{d}{d R}(U / R)+\frac{2 G}{R}\left(\frac{d U}{d R}+\frac{U}{R}\right)=0
$$

The solution to Equation $(3.4 .1)$ is

$$
U=f R+\frac{g}{R}
$$

which gives

$$
\begin{aligned}
& \varepsilon_{R}=\frac{d U}{d R}=a-\frac{b}{R^{2}} \\
& \varepsilon_{\theta}=\frac{U}{R}=a+\frac{b}{R^{2}}
\end{aligned}
$$

where $f$ and $g$ are constants evaluated from initial conditions. Evaluating a and D from initial conditions

$$
\begin{aligned}
& a=\frac{R_{0}^{2} \Delta P_{0}-R_{1}^{2} \Delta P_{1}-\lambda \varepsilon_{z}\left(R_{1}^{2}-R_{0}^{2}\right)}{2(\lambda+G)\left(R_{1}^{2}-R_{0}^{2}\right)} \\
& b=\frac{\left(\Delta P_{0}-\Delta P_{1}\right) R_{0}^{2} R_{1}^{2}}{2 G\left(R_{1}^{2}-R_{0}^{2}\right)}
\end{aligned}
$$

when

$$
\Delta P_{0}=P_{0}-P_{0}^{0}
$$

and

$$
\Delta P_{1}=P_{1}-P_{1}^{0}
$$

If the initial condition is unstressed then 


$$
\Delta P_{0}=P_{0}
$$

and

$$
\Delta P_{1}=P_{1}
$$

For the inner cylinder depicted in Figure $3-7$

$$
U\left(R_{1}\right)=-\frac{P_{1} R_{1}}{2\left(R_{1}^{2}-R_{0}^{2}\right)}\left[\frac{R_{1}^{2}}{\lambda+G}+\frac{R_{0}^{2}}{G}\right]-\frac{\lambda \varepsilon_{2} R_{1}}{2(\lambda+G)}
$$

and the cladding cylinder

$$
\begin{aligned}
U^{\prime}\left(R_{2}\right)= & \frac{P_{3} R_{2}}{2\left(R_{3}^{2}-R_{2}^{2}\right)}\left[\frac{R_{2}^{2}}{\lambda^{\prime}+G^{\prime}}+\frac{R_{3}^{2}}{G^{-}}\right] \\
& -\frac{P_{2} R_{2}}{2\left(R_{3}^{2}-R_{2}^{2}\right)}\left[\frac{R_{3}^{2}}{\lambda^{\prime}+G^{-}}+\frac{R_{3}^{2}}{G^{-}}\right]-\frac{\lambda^{\prime} \varepsilon_{2}^{\prime} R_{1}}{2\left(\lambda^{\prime}+G^{-}\right)}
\end{aligned}
$$

Since the cladding layer is of zero strength, $P_{1}=p_{2}$. Substituting $p_{1}$ for $P_{2}, P$ for $P_{3}$, and letting $\varepsilon_{2}=\varepsilon^{\prime}{ }_{2}=0$ (constant stress in axial direction), then $J\left(R_{1}\right)$ $=U^{\prime}\left(R_{2}\right)$ and

$$
P_{1}=\frac{P R_{2} R_{3}\left[\frac{1}{\lambda^{\prime}+G^{2}}+\frac{1}{G^{2}}\right]}{2\left(R_{3}^{2}-R_{2}^{2}\right)\left[\frac{R_{1}}{2\left(R_{1}^{2}-R_{0}^{2}\right)}\left(\frac{R_{1}^{2}}{\lambda+G}+\frac{R_{0}^{2}}{G}\right)+\frac{R_{2}}{2\left(R_{3}^{2}-R_{2}^{2}\right)}\left(\frac{R_{2}^{2}}{\lambda^{2}+G^{-}}+\frac{R_{3}^{2}}{G^{2}}\right)\right]}
$$

which gives the interface pressure $P_{1}$ as a function of the repository pressure ${ }^{2}$ ? and material properties of backfill and structural wall. 
The pressure on the inner boundary of the backfill determines whether or not the backfill will remain as an elastic wall or flow plastically. It is assumed that the outer pressure is the repository pressure. When the inner pressure falls below a threshold value the backfill yields and flows plastically. That is, there is a maximum pressure gradient that the backfill will support. Exceeding this gradient is indicated by a minimum pressure at the inner boundary of the backfill since the outer pressure is maintained constant at repository pressure. Once the minimum is reached yield is triggered, the backfill flows olastically and the interface pressure rises to equal the repository pressure. The backfield yield triggering is given by

$$
P_{1} \leq \frac{P_{R}(1-n)}{\left(1-n \frac{R_{2}^{2}}{R_{3}^{2}}\right)}
$$

where

$$
n=\sqrt{\frac{4}{27} \beta^{2}(1-v)^{2}-\frac{1}{3}(1-2 v)^{2}}
$$

Note that $n$ becomes imaginary if

$$
\beta<1.5 \frac{(1-2 v)}{(1-v)}
$$

If $n$ is imaginary, the backfill will yield plastically for any value of $p_{1}$. It is therefore a minimum condition that the backfill have $a \beta$ and $v$ such that

$$
3>1.5 \frac{(1-2 v)}{(1-v)}
$$

iypical vaiues for 3 and $v$ have been found in the literature and range from approximately $0.6-1.2$ and $0.25-0.45$, respectively. 
In the case of wall yield the inside pressure is neglected as it is very small compared to repository pressure. Thus yielding is controlled by the pressure at the outside of the wall. The condition for no yield established for cylindrical shells is

$$
v^{2}>4\left[\frac{P_{1}}{1-\frac{R_{0}^{2}}{R_{1}^{2}}}\right]^{2}\left(1-v+v^{2}\right)
$$

Thus yield will occur when

$$
P_{1} \geq \frac{\gamma}{2} \frac{1-\left(R_{0}^{2} / R_{1}^{2}\right)}{\sqrt{1-v+v^{2}}}
$$

Internal pressure stress was based on ASME code requirements. Hoa stress criteria from ASiE Code, Section VIII, Division 1 (AslE, 1977) give

$$
\delta=\frac{P_{0} P_{0}}{\left(S E-0.6 P_{0}\right)}
$$

where

= wall thickness, (in)

$E=$ joint efficiency for longitudinal seam

(=1 for seamless or full penetration weld)

$S=$ allowable stress for material, (psi)

$P_{0}=$ internal pressure, (Dsi)

$R_{0}=$ inside radius, $(i n)$ 
The allowable stress tables from the code were used to develop a correlation for $S$ as a function of temperature, $T$. The subroutine calculates $S$ from

$$
\begin{array}{ll}
S=S T-S 2(T) & T>S 3 \\
S=S 4 & T \leq S 3
\end{array}
$$

Solving Equation (3.4.44) for $P_{0}$

$$
P_{0}=\frac{\delta S E}{R_{0}+0.6 \delta}
$$

When

$$
P_{0}>\frac{\delta S E}{R_{0}+0.6 \delta}
$$

then WFAIL is set to 0 , meaning the wall has failed.

The STRESS subroutine is called by the main program at each time increment for each barrier layer. The subroutine determines whether the current barrier under consideration remains intact or fails at that time increment. Nall thickness is the current value returned from the corrosion subroutine CORODE.

Figure $3-8$ is a flowchart of the subroutine. The subroutine first checks to see if external or internal pressure are of concern. The value of CREEP is then "YES" Or "NO". If CREEP = YES then the external routine is used, otherwise internal pressure is checked against the code criteria. If external pressure is of concern then radii are calculated based on the latest value of THICK, the wall thickness. Presence of a backfill is checked. If there is no backfill then the pressure on the outside of the wall ("interfacial" pressure EPRESS) is set equal to repository pressure REPRES. The next step is to check for backfill failure. If the backfill yields then EPRESS = REPRES. If the backfill has not failed then calculations are carried out to determine the status of the backfil1. If failure is determined then once again EPRESS = REPRES. Then the subroutine calculates stresses on the wall as interfacial pressure EPRESS and checks for wall yield. AFAIL is set to 1 if the wall is intact or D if failure 


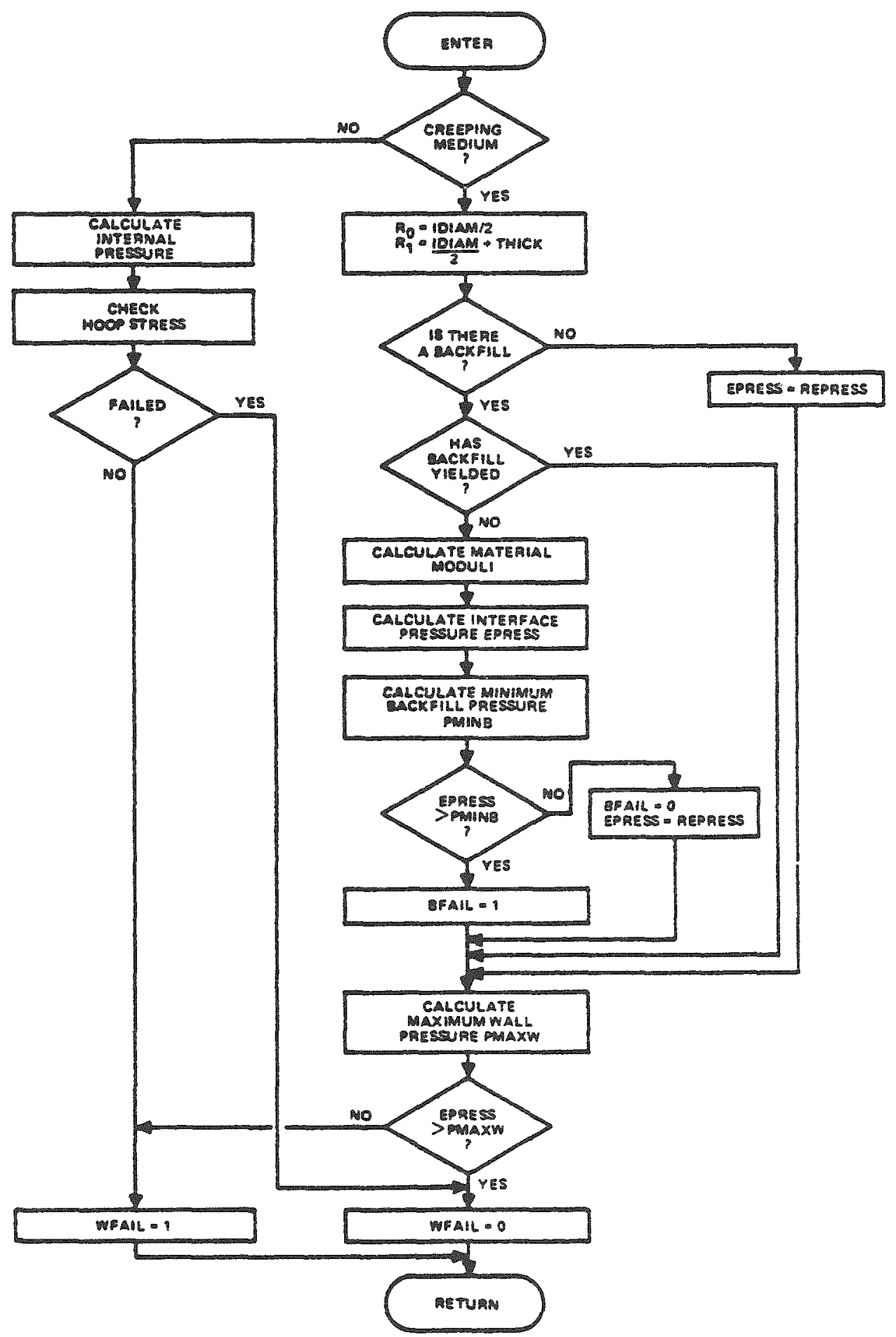

Figure 3-3. STRESS Flowchart. 
is determined. Based on the value of dFAIL the main program continues with this barrier or proceeds inward.

\subsection{RADIONUCLIDE RELEASE RATES}

The radionuclide release model RELEAS calculates the transport rate of specific radionuclides through failed engineered barriers and backfill. The specific rate of interest is the release rate to the geology. The model is based on slab geometry which is a conservative assumption relative to a cylindrical geometry. The engineered barrier package can consist of many layers of different materials. At some time after emolacement in the repository the barriers fail, either by crushing from the lithostatic pressure in the repository or by corrosion. In either case, when the barriers fail, it is assumed that water is available throughout the fuel bundle, barriers, and backfill; and mass transport by diffusion begins.

The objective of the radionuclide release nodel is to calculate the release rate based on Fick's second law of diffusion, i.e.., no countercurrent diffusion and no convection of water. The question of water convection was discussed in section 2 under the high water flow rate scenario. The backfill is assumed to have capacitance in excess of that of a solution. The capacitance is due to sorption of the species of interest. Resistance to mass transfer is al so assumed to exist because of the remains of the failed barriers. This assumption is reasonable because there is a finite distance from the waste to the backfill face, and the failed barriers represent a physical resistance through a void fraction available for transport, i.e., a porous barrier. The failed engineered barrier is assumed to have no capacitance since the capacitance of the backfill is much larger.

In the model description that follows the waste resides next to the backfill slab at $x=2$. A zero-capacitance mass transfer pesistance is assumed to be present at $x=\ell$, similar in concept to a heat transfer coefficient, and a mass transfer resistance is assumed to be present at the Dackfill-geologs interface $(x=0)$ that is $1 / 10$ of that $2 t x=2$. The geology is assumed to sweep away the radionuclides as soon as they arprive at $x=0$ so that the boundary condition at $x=0$ is a zero concentration. Wote that the results are relatively insensitive to resistance at the geology face and often insensitive to failed barrier resistance (except for cases with little or no backfili). 
Consider a slab of thickness 2 with the conductance boundary conditions

$$
\begin{array}{ll}
\frac{\partial c}{\partial t}=k \frac{\partial^{2} c}{\partial x^{2}} & 0<x<\ell \\
\frac{d c}{d x}+h_{\ell}\left(c-c_{\ell}\right)=0 & \text { at } x=\ell \\
\frac{d c}{d x}-h_{0} c=0 & \text { at } x=0 \\
\text { and } c(x, 0)=0 &
\end{array}
$$

These equations describe the time-dependent diffusion phenomenon with so-called "radiation" boundary conditions (Carslaw, 1967). Since diffusion is assumed to be occurring through a porous medium, the constant $k$ is not the liquid diffusion coefficient.

In considering diffusion through a porous medium, an effective diffusivity is (Bird, 1965) (Smith, 1970)

$$
N_{a} \equiv-D_{e} \frac{\partial C}{\partial x}
$$

where $c$ is the concentration of the species of interest contained in the liquid volume only, not the total unit volume including solid. $N_{a}$ is the flux oer actual unit area and $D_{e}$ is measured experimentally. The effective diffusivity for a porous medium is estimated to be

$$
D_{e} \equiv \frac{\varepsilon D_{2}}{\delta}
$$

where $\varepsilon$ is the void volume and $\delta$ is the tortuosity. Therefore, the effective diffusivity is defined in terms of liquid concentrations. 
Transient diffusion is described by

$$
D_{e} \frac{\partial^{2} c}{\partial x^{2}} \equiv \text { Accumulation }
$$

Since the diffusive flux is based on liquid concentration there is accumulation with no adsorption

$$
D_{e} \frac{\partial^{2} c}{\partial x^{2}} \equiv \varepsilon \frac{\partial c}{\partial t}
$$

When accumulation by adsorption also occurs, another term must be added to account for it.

The amount of naterial adsorbed on the solid of the porous medium is obtained from information on the equilibrium constant

$$
c_{s} \equiv k_{d} c
$$

where $c_{s}=$ grams of species of interest adsorbed on one gram of solid, so that units are

$$
k_{d}[=]\left[\frac{g m s \text { on solid }}{g m \text { solid }}\right]\left[\frac{\mathrm{ml} \text { of liquid }}{\mathrm{gm} \text { in liquid }}\right]
$$

Hence, $k_{d}$ is reported as $\pi l / 3 m$. How if $o$ is the bulk density of the solid, $2 C_{5}$ yields the amount of adsorbed material in equilibrium with the liquid concentration $c$ or

$$
D_{e} \frac{\partial^{2} c}{\partial x^{2}}=\left(\varepsilon+k_{d} p\right) \frac{\partial c}{\partial t}
$$


Therefore,

$$
k \equiv \frac{D_{2}}{\delta\left(1+\frac{k_{d}^{\rho}}{\varepsilon}\right)}
$$

where

$$
\begin{aligned}
& k=\text { constant in Equation }(3.5 .1),\left(\mathrm{cm}^{2} / \mathrm{yr}\right) \\
& \rho=\text { bulk density of the solid, }(\mathrm{gm} / \mathrm{m}) \\
& k_{d}=\text { distribution constant, }(\mathrm{ml} / \mathrm{gm})
\end{aligned}
$$

The boundary condition in Equation (3.5.2) contains the conductance $h_{l}$ which is derived from

$$
D_{e} \frac{d c}{d x}+H_{\ell}\left(c-c_{\ell}\right)=0 \quad \text { at } x=2
$$

where it is seen that

$$
h_{2}=\frac{H_{e}}{D_{e}}
$$

$\mathrm{H}_{2}$ is called a conductance since it multiplies a gradient rather than a flux and is assumed to be the result of diffusion through a distance $\Delta x$ with no capacitance. Examining a slab for this assumption yields

$$
q(\Delta x)=D(\Delta c)
$$

where $q$ is the flux, $\Delta x$ is the slab thickness, 0 is the diffusion coefficient, and $\Delta c$ is the concentration difference. Therefore, for the problem considered here

$$
H_{x}=\frac{D}{3 x}
$$


where $D$ is the diffusivity through the medium in $3 x$ which is the corroded barrier.

The solution to Equation (3.5.1) with the prescribed boundary and initial conditions is of the form

$$
c(x, t)=w(x, t)+w(x)
$$

where $u(x)$ is the steady state solution and is

$$
u(x)=\frac{c_{2} h_{2}}{1+h_{2}\left(2+\frac{1}{h_{0}}\right)}\left(x+\frac{1}{h_{0}}\right)
$$

and the transient solution is

$$
w(x, t)=\sum_{n=1}^{\infty} A_{n} x_{n} \exp \left(-k a_{n}^{2} t\right)
$$

and

$$
x_{n}=\cos \left(z_{n} x\right)+\frac{n_{0}}{a_{n}} \sin \left(\nu_{n} x\right)
$$

where $x_{n}$ is the n-th Dositive root of

$$
\tan (\alpha z)=\frac{\alpha\left(h_{0}+h_{2}\right)}{a^{2}-h_{x_{0}}}
$$

and 


$$
A_{n}=\frac{2 a_{n} c_{2} n_{2}\left\{\frac{n_{0}^{2}}{a_{n}} \cos \left(a_{n}^{2)}-\left[2+\frac{n_{0}}{a_{n}^{2}}+\frac{1}{n_{0}}\right] \sin \left(\alpha_{n} \ell\right)\right\}\right.}{\left\{\left(a_{n}^{2}+n_{0}^{2}\right)_{2}+n_{\ell}\left(\frac{a_{n}^{2}+n_{0}^{2}}{a_{n}^{2}+n_{2}^{2}}\right)+n_{0} \mid\left[1+n_{\ell}\left(2+\frac{1}{n_{0}}\right)\right]\right.}
$$

A special case of the conditions described by Equations (3.5.1) (3.5.4) is considered where the conductances are large, i.e., the resistances are close to zero. This case is illustrated in Figure 3-9 where line A represents the steady state solution for $h_{2}=h_{0} \rightarrow \infty$, and line $B$ represents the steady state solution for $h_{2}$ and $h_{0} \ll \infty$ Experience indicates that when " 3 " approaches " $A$ " the solution described by Equations (3.5.17) - (3.5.22) converges very slowly and is impractical to evaluate. A solution with fixed boundary conditions should be used when

$$
\frac{c_{1}-c_{2}}{c_{i}}=A>0.8
$$

is satisfied.

The solution with fixed boundary conditions and zero initial condition is (Carslaw, 1967)

$$
c(x, t)=\frac{2 c_{l}}{\pi} \sum_{n=1}^{\infty} \frac{(-1)^{n}}{n} \sin \left(\frac{n \pi x}{2}\right) \exp \left[-k\left(\frac{n \pi}{2}\right)^{2} t\right]+c_{2} \frac{x}{L}
$$

From the equations described, the quantity of material transported across the boundaries at $x=0$ and $x=2$ can be calculated during the transient period. When steady state is attained, the much simpler steady state solutions yield the quantity transported. The time at which steady state is attained is defined to be when the lead exponential in Equation (3.5.19) has decayed to 0.01 , or

$$
t_{s}=\frac{4.6}{k_{i}^{2}}
$$

or using Equation (3.5.24) 


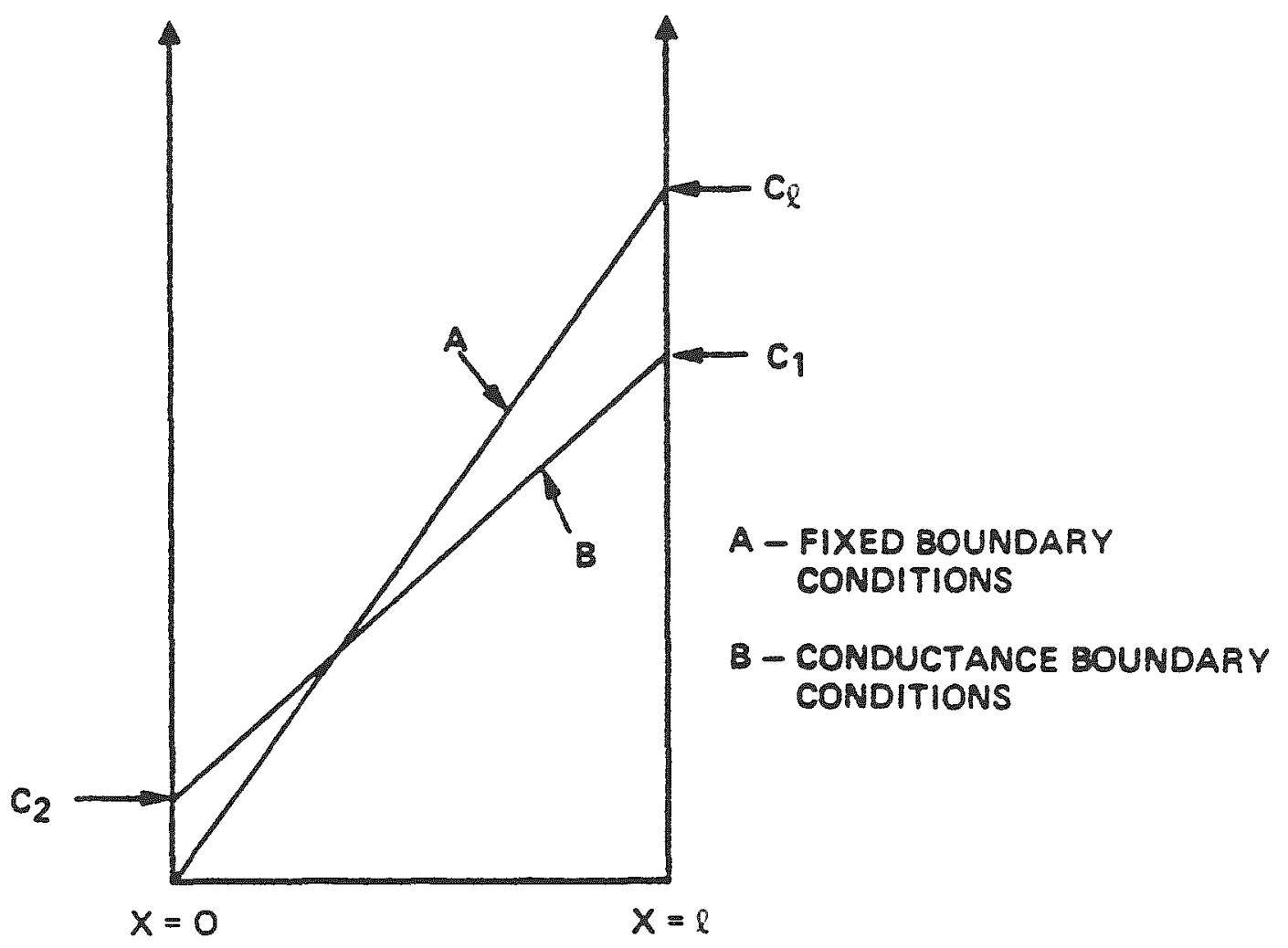

Figure 3-9. illustration of Steady Siate Solution. 


$$
t_{s}=\frac{4.6}{k(\pi / L)^{2}}
$$

The transport of radionuclides occurs only as long as there is material remaining in the waste. A material balance around the waste for a specific radionuclide yields

$$
\frac{d y}{d t}=-f(t)-\lambda y
$$

where $y$ is the quantity of material in the waste at any time $t, \lambda$ is the decay constant, and $f(t)$ is the rate of transport of material out by diffusion as described by Equations (3.5.17) or (3.5.24). The result for integrating the linear first order differential Equation (3.5.27) where $f(t)$ is described by Equation (3.5.17) is:

$$
\begin{aligned}
y(t)= & H_{l}^{0} \sum_{n=1}^{\infty} \frac{B_{n}}{\left(\lambda-k \alpha_{n}^{2}\right)} \exp \left(-k \alpha_{n}^{2} t\right)+\frac{k_{l}}{\lambda} \\
& +\exp (-\lambda t)\left[y_{0}-H_{l}^{0} \sum_{n=1}^{\infty}\left(\frac{B_{n}}{\lambda-k \alpha_{n}^{2}}\right)-\frac{k_{l}}{\lambda}\right]
\end{aligned}
$$

where

$$
\begin{aligned}
& y_{0}=\text { initial quantity of material } \\
& H_{\ell}^{0}=a H_{\ell} \\
& a=\text { area availabie for transport at } x=2 \\
& K_{2}=H_{2}^{0}\left[c(2)-c_{2}\right] \\
& B_{n}=A_{n} x_{n}(x=l)
\end{aligned}
$$

Likewise for Equation (3.5.24) 


$$
\begin{aligned}
y(t)= & -2 k \sum_{n=1}^{\infty} \frac{\exp \left[-k\left(\frac{n \pi}{l}\right)^{2} t\right]}{\left[\lambda-k\left(\frac{n \pi}{l}\right)^{2}\right]}-\frac{k}{\lambda} \\
& +\exp (-\lambda t)\left[y_{0}+2 k \sum_{n=1}^{\infty} \frac{1}{\left[\lambda-k\left(\frac{n \pi}{l}\right)^{2}\right]}+\frac{k}{\lambda}\right]
\end{aligned}
$$

where

$$
K=2 D_{e} c_{2} / 2 .
$$

In the event there is sufficient material at $t=0$ to attain steady state, a material balance on the waste for a specific radionuclide yields

$$
\frac{d z}{d t}=-\lambda z-r_{t r}
$$

where $r_{t r}$ is the constant rate of material transported out by diffusion. Integrating and solving for the time when the quantity of material in the waste is zero yields

$$
t_{f}=\frac{1}{\lambda} \ln \left[\frac{\lambda z_{0}+r_{t r}}{r_{t r}}\right]
$$

where $z_{0}$ is the quantity of material present when steady state is attained and $t_{f}$ is the time beyond the steady state time required for $z=0$.

The transport of radionuclides through an adsorbing medium can be calculated using Equation (3.5.17) for the case when surface conductances are present or Equation (3.5.24) for the case of fixed boundary conditions. The constant $k$ is calculated from Equation (3.5.12) for both Equations (3.5.17) and (3.5.24). The use of Equation (3.5.17) or (3.5.24) is determined by Equation (3.5.23). The steady state times are calculated by Equation (3.5.25) or (3.5.25). The quantity of a specific radionuclide remaining in the waste at any time, $t$, is calculated from Equation (3.5.28) or $(3.5 .29)$. In the event $y_{0}$ is not large enough to allow steady state transport to be attained, Equation (3.5.28) or (3.5.29) is solved by trial and error to find the time where $y=0$. If steady state is attained, the additional time required for radionuclides in the waste to attain zero quantity is calculated from Equation (3.5.31). The 
calculations essintially stop when $y=0$, whether this is before or after steady state. There are no equations derived here for the transoort across the $x=0$ boundary afta $y=0$. This "tail" is ignored. Furthemore, radiodecay is not considered for material in the region $0 \leq x \leq 2$. For the radionuclides considered, such as an ricium-241, ignoring radiodecay does not result in an appreciaole error in predicting transport rates as will be shown in the following discussion. Cases where radiodecay must be considered will be noted. A materiâl balance over a differential thickness in a slab when radiodecay is considered yields

$$
D_{e} \frac{\partial^{2} c}{\partial x^{2}}=\left(\varepsilon+k_{d} p\right) \frac{j c}{\partial t}+s i c
$$

Thus

$$
\frac{\partial^{2} c}{\partial z^{2}}-b c-\frac{1}{k} \frac{\partial c}{\partial t}=0
$$

where $z=x / 2$ and

$$
b=\frac{i^{2} i \varepsilon}{D}
$$

A solution is available for the case where the radio-diffusion parameter, $D$, is zero as described by Equations (3.5.1) - (3.5.4) and Equation (3.5.19). The solution to Equation (3.5.33) is (Janckiwerts, 195i)

$$
c(x, t)=k b \int_{0}^{t} e^{-k b t^{\prime}} c_{0}\left(x, t^{\prime}\right) d t^{\prime}-e^{-k b t} c_{0}(x, t)
$$

where $c_{0}(x, t)$ is the $b=0$ solution. 
The above equation is valid for boundary conditions of constant concentration or "radiation", but only with a zero initial condition. Applying Equation $(3.5 .35)$ to Equation $(3.5 .19)$ yields

$$
\begin{aligned}
c(x, t) & =\sum_{n=1}^{\infty} A_{n}\left[\cos x_{n} x+\frac{h_{0}}{x_{n}} \sin \left(x_{n} x\right)\right] . \\
& \cdot\left[\frac{b+k a_{n}^{2} \exp \left[-\left(b+k \alpha_{n}^{2}\right) t\right]}{b+k a_{n}^{2}}\right]
\end{aligned}
$$

where $A_{n}$ and $\alpha_{n}$ are defined in Equations (3.5.21) and (3.5.22).

The above equation is inconvenient to use in examining the effects of radiodecay, and the same probiem examined with fixed boundary conditions rather than "radiation" is more instructive.

Solving Equation $(3.5 .33)$ with

$$
\begin{aligned}
& c(0, t)=0 \\
& c(x, t)=1 \\
& c(x, 0)=0
\end{aligned}
$$

yields

$$
c(z, t)=\frac{2}{T} \sum_{n=1}^{\infty} \frac{(-1)^{n}}{n} \sin \lambda_{n} z\left[\frac{b+\lambda_{n}^{2} \exp \left[-\left(b+i_{n}^{2}\right) t\right]}{b+i_{n}^{2}}\right]+z
$$

where $i_{n}=n-$

The steady state solution can be obtained from the above equation for $t=x$, but a simpler form is obtained by solving Equation (3.5.33) with $3 c / 3 t=0$. Doing this yields 


$$
c(z)=\frac{\sinh v \sqrt{b} z}{\sinh v \sqrt{b}}
$$

Which gives the same results as Equation (3.5.40) for $t=\infty$. Equation (3.5.41) is presented in Figure 3.10 for various values of the radio-diffusion parameter, $b$. Figure 3-10 clearly shows that when $b<1$ the effect of radiodecay on the diffusion transport rate is negligible. In studying the diffusion transport of isotopes such as americium-241 with a decay constant, $\lambda$, of $1.5 \times 10^{-3}$ year $-1,0=$ $31.5 \mathrm{~cm}^{2} /$ year and $s=4$,

$$
b=1.9 \times 10^{-4} 2^{2}
$$

with $z$ in centimeters. Hence, in order for $b$ to be $<1$, \& must be $72.5 \mathrm{~cm}(28$ inches) or less. This value of 2 is considerably larger than any of those used in the case studies presented in this study and therefore is the justification for ignoring radiodecay in the diffusion transport calculations. Ignoring radiodecay results in a conservatively high computed geological release rate at the $z=0$ boundary.

If shorter-ijved radionuclides are of interest, such as cesium-137 with $a A=0.023$ year ${ }^{-1}$, then for $b<1,2$ must be $18.5 \mathrm{~cm}(7.3$ inches) or less in order to use the $b=0$ diffusion transport equations.

The results presented in Figure $3-10$ clearly show how the release rate at $z=0$ is retarded by the effect of residence time in the $s$ lab and radiodecay. The ratio of the transport rate at $z=0$ to that at $z=1$ is called the retard factor, $R_{f}$, and is calculated from

$$
R_{f}=1 / \cosh \vee 5
$$

Values of $R_{f}$ are presented in Table 3-5 for various values of the radio-diffusion parameter, $b$. Also tabulated is the approximate number of times the transport rate at $z=0$ is halved relative to that at $z=1$. Hence, for $b=100$, the transport rate at $z=0$ is $2^{-13}$ of that at $z=1$. In order to have $0=100$ for americium-241 with $D=31.5 \mathrm{~cm}^{2} /$ year and $\delta=4$, i must be $725 \mathrm{~cm}$ or approximately 24 feet. 


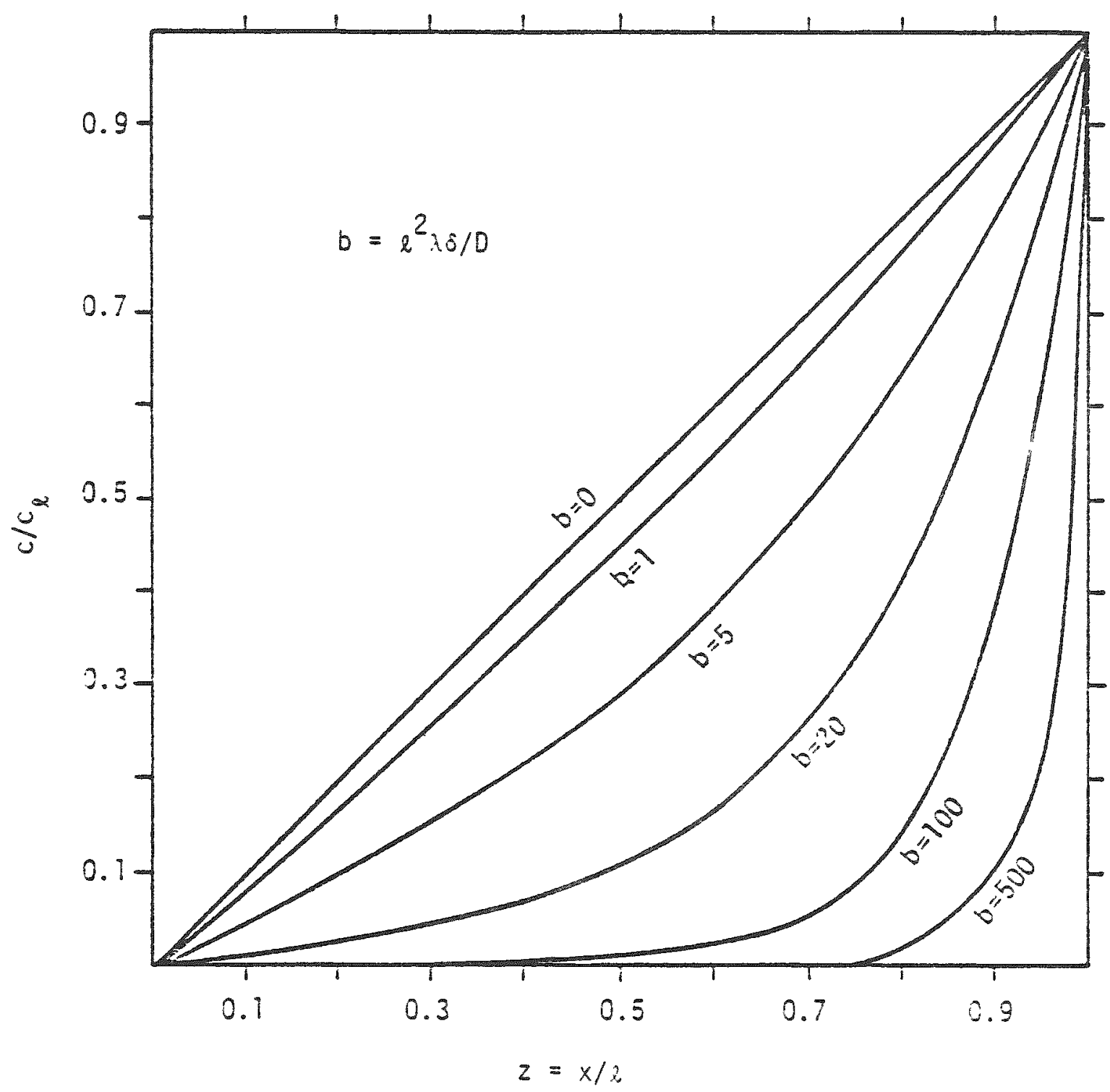

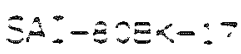

Figure 3-10. Steady State Concentration Profiles in a Slab for Values of the Radio-Diffusion Parameter, $b$. 
Table 3-6. Retardation of the Transport of Radionuclides Through a Slab Barrier at Steady State for Values of the Radio-Diffusion Parameter, $b$.

\begin{tabular}{|c|c|c|}
\hline $\begin{array}{c}\text { Radio-Diffusion } \\
\text { Parameter } \\
\text { b }\end{array}$ & $\begin{array}{c}\text { Transport Retard } \\
\text { Factor } \\
R_{f}\end{array}$ & $\begin{array}{c}\text { Number of Transport } \\
\text { Half-Lives Retarded } \\
n\end{array}$ \\
\hline & & - \\
0 & 1 & - \\
1 & 0.65 & 2.2 \\
5 & 0.2 & 5.4 \\
100 & 0.02 & 13 \\
500 & $9 \times 10-5$ & 31 \\
\hline
\end{tabular}


The approach to the steady state concentration profiles as a function of the radio-diffusion parameter is described by Equation $(3.5 .40)$. Note $b$ always appears with $\lambda_{n}^{2}$ as $b+\lambda_{n}^{2}$. Since $\lambda_{n}=n \pi$, for large $n, b<\lambda_{n}^{2}$. For the radionuclides examined in this study $b$ is always $\ll \lambda_{1}$.

In order to justify using Equation $(3.5 .40), b$ should be $>1$ and preferably on the order of 10. The evaluation of the series in Equation (3.5.40) is straightforward with a typical result presented in Figure 3-11 for $b=10$. This figure shows that the approach to the steady state concentration profile with respect to time is similar to the results for $b=0$, i.e., the high frequency Fourier components decay away very rapidly, and when $\theta>0.1$, the concentration profile is essentially at steady state.

There are two types of leaching that must be considered when developing a mathematical description for the purpose of obtaining diffusion coefficients from data or for predicting future behavior of leaching systems. The first is the situation where the soluble substance is in solution within the solid matrix at the start of the transport. For a semi-infinite slab containing a dissolved substance in the solution in its pores the defining equations are

$$
k_{c} \frac{\partial^{2} c}{\partial x^{2}}=\frac{\partial c}{\partial t} \quad 0<x<\infty
$$

and

$$
\begin{aligned}
& c(x, 0)=c_{0}(x) \\
& c(0, t)=0 \\
& c(\infty, t)=c_{\infty}
\end{aligned}
$$

where

$$
k_{c}=a \text { constant (mass transfer "conductivity") }
$$

In the above case the soluble material is removed as fast as it arrives at the $x=0$ face. In the event the solution at the face is not zero or there is an equilibrium condition, the equations are still easily solved.

The second type of leaching is the case where the soluble substance exists in the solid matrix as a solid. The soluble substance must be first dissolved and then diffused out of the solid. Neglecting the transport of 


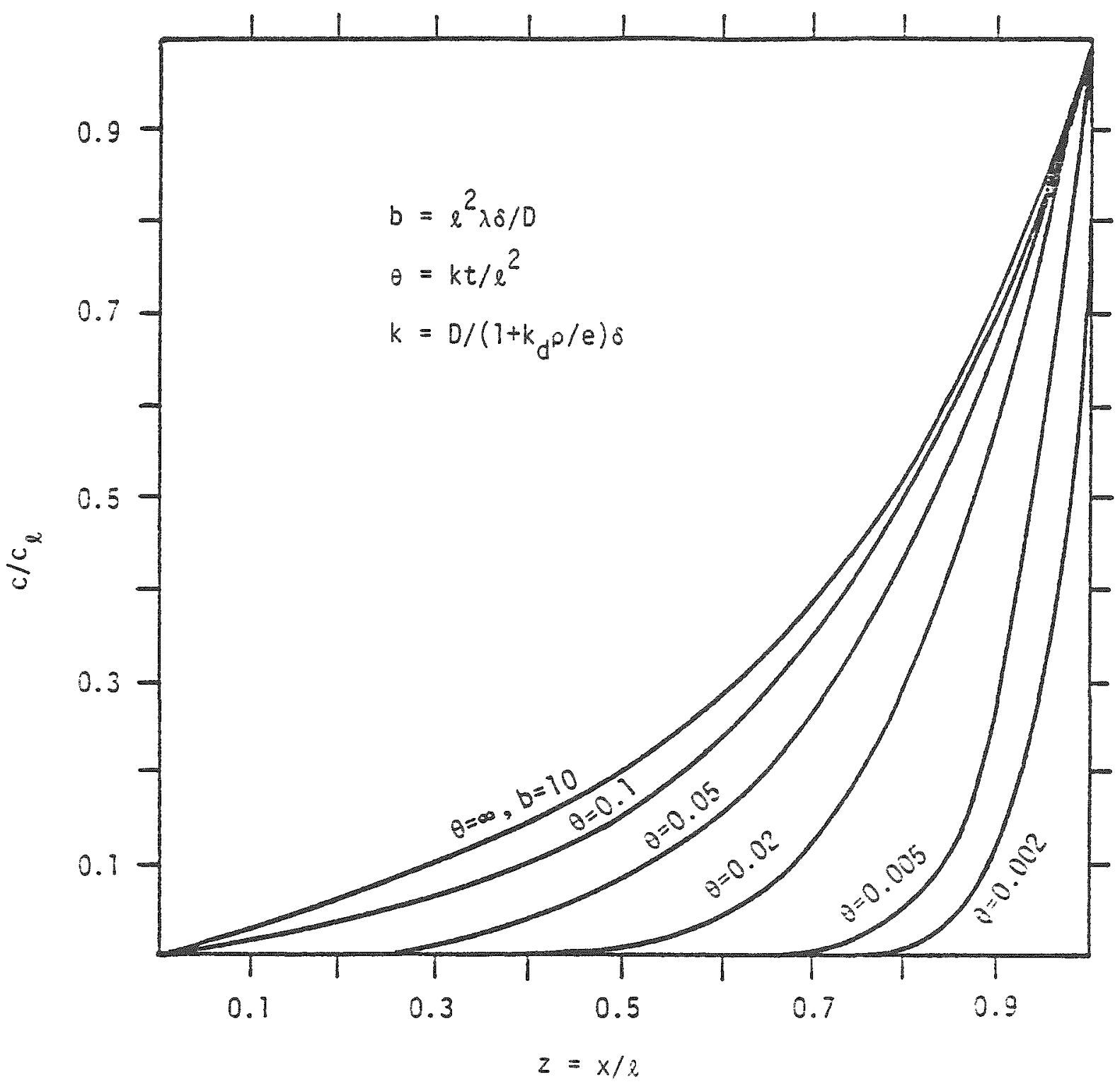

$\operatorname{Sin} 1-808 K-: 3$

Figure 3-11. Concentration Profiles for the Approach to Steady State in a Slab. 
solvent into the matrix, this leads to a condition at the soluble substance-solution interface in the solid that is expressed by

$$
\left.D_{e} \frac{d c}{d x}\right|_{x=z_{s}}=\rho_{s} \frac{d z_{s}}{d t} \text { (at the interface) }
$$

where $z_{s}$ is the location of the soluble substance-solution interface and $z_{s}$ is the apparent density of the soluble substance.

The mathematical solution to the latter type of leach problem is similar to the heat transfer problem where a change of state occurs. There are some solutions available for the semi-infinite slab but there appear to be no closed solutions for the important boundary conditions of constant flux. In the case of cylindrical coordinates there is only one simple exact solution for a continuous line source of heat. (Carslaw, 1967).

In examining the available leach data (Katayama, 1976, 1980) for the radionuclides of interest, it is determined that the latter type of leaching is the type occurring. As a result, it was determined that there is not straightforward way to reduce the available data to obtain a diffusivity for the radionuclides of interest. The only practical way the available leach data could be used is to specify a boundary condition either with a flux or a concentration as determined by the available data.

The data are published as a flux based on available spent fuel surface area. Also available in this published data are solution concentrations which were used to obtain the flux data. Therefore, the choice existed to use the daca for a flux or a concentration boundary condition. In this study a concentration boundary condition was chosen rather than a flux condition because a flux specification can result in a concentration which is unrealistically large, i.e., the solubility limit is exceeded and unrealistic results can be obtained. The concentration build-up can occur because mass is not transferred away fast enough from the face where the flux is specified. Only after the concentration has increased enough in the immediate vicinity to yield a gradient large enough will the concentration stabilize.

Only one of the published repnrts (Katayama, 1976) yields sufficient experimental detail to obtain concentration information. These data are used to specify a fixed concentration boundary condition, i.e., not a function of time. 
This is in contrast to the published results where the concentration in solution is quite high in the initial experiments. However, the initial effects do not last over very many days of leaching. Since the data are to be used to predict transport rates over hundreds to thousands of years, it is considered justifiable to use a leach concentration that appears more constant after many leaching solution exposures. Also, because of the nature of the adsorption of the backfill in the model, any "front-end" effect in concentration is quickly adsorbed.

It appears that further study of the leaching data, how to use these data to obtain transport coefficients, and the type of model that can use the data is warranted. In examining the leaching data it was found that usually less than one percent of the leachable radionuclides are ever renoved from the spent fuel. In this case all the data should be considered as initial phenomena, and extrapolating this information to predict leaching behavior where greater portions are transported is difficult unless the transport orocess is properly described, i.e.s dissolution and diffusion in porous media. Also to be considered is the possibility of adsorption occurring on the $\mathrm{UO}_{2}$ matrix which would alter the defining mathematics. However, without proper problem definition simple extrapolation of existing data must be used in mathematical models that describe the transport of radionuclides.

In the radionuclide release model the ralease of radionuclides to the geology is set to zero at the time the inventory of a radionuciide in the canister becomes zero. This is equivalent to deleting the tail-off of the band breakthrough curve as illustrated in Figure $3-12$ (shaded area). While it is possible to calculate the tail-off, it is an inordinately time-consuming calculation and is deemed inappropriate for the purposes of the performance model. However, the method of calculation is discussed in this section.

when the inventory of the radionuclide of interest reaches zero in the waste package, the boundary condition at the backfill-can interface is assumed to becone insulated. The equations to solve in this case are

$$
\begin{gathered}
\frac{\partial c}{\partial t}=k \frac{\partial^{2} c}{\partial x^{2}} \\
\frac{d c}{d x}-h_{0} c=0 \quad \text { at } x=0
\end{gathered}
$$




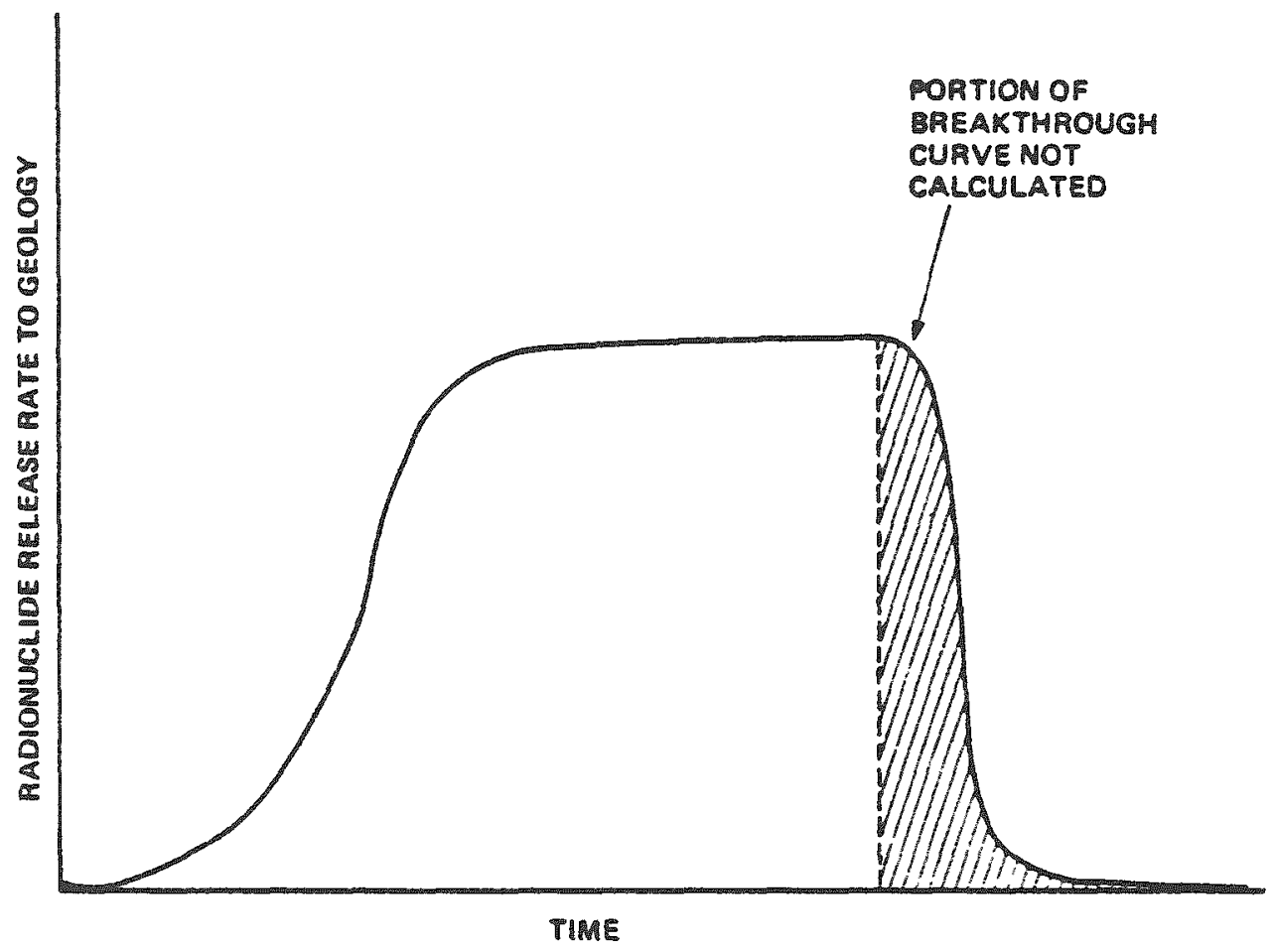

Figure 3-12. Deletion of Breakinrough Tail of Radionuclide Release Rate to Geology. 


$$
\frac{d c}{d x}=0 \quad \text { at } x=\hat{x}
$$

and

$$
v(x, 0)=f(x)
$$

where $f(x)$ is the concentration profile in the backfill when the zero-inventory condition occurs. Hence, $f(x)$ is Equation (3.5.19) for some value of $t=t_{0}$. The solution for Equation $(3.5 .49)$ is

$$
\begin{aligned}
& c(x, t)=\sum_{j=1}^{\infty}\left(\frac{2}{\ell\left(s_{j}^{2}+h_{0}^{2}\right)+h_{0}}\right) \exp \left[-k B_{j}^{2}\left(t-t_{0}\right)\right] \cdot \\
& -\left\{\frac{c_{0} h_{2}}{1+l h_{l}+h_{2} / h_{0}}\left[\left(l+\frac{1}{h_{0}}+\frac{h_{0}}{\beta_{j}^{2}}\right) \sin \beta_{j} 2-\frac{h_{0} l}{\beta_{j}} \cos \beta_{j} l\right]\right. \\
& +\sum_{n=1}^{\infty} \frac{\frac{2 c_{0} h_{2}}{1+2 h_{2}+h_{2} / h_{0}}\left[\frac{h_{0} l}{a_{n}} \cos a_{n} \ell-\left(l+\frac{1}{h_{0}}+\frac{h_{0}}{a_{n}^{2}}\right) \sin a_{n}^{2}\right]}{\left(a_{n}{ }^{2}+h_{0}{ }^{2}\right)\left[\ell\left(a_{n}{ }^{2}+h_{2}{ }^{2}\right)+h_{2}\right]+h_{0}\left(a_{n}{ }^{2}+h_{2}^{2}\right)} \text {. } \\
& \text { - } h_{i}\left(\alpha_{n}^{2}-3_{j}^{2}\right)\left(x_{n} \cos x_{n}^{2}+h_{0} \sin \alpha_{n}{ }^{2}\right)\left(z_{j} \cos \beta_{j} i+h_{0} \sin z_{j} i\right) \text {. } \\
& \left.\left.\cdot \exp \left(-k \alpha_{n}^{2} t_{0}\right)\right|_{j} \cos 3_{j} x+h_{0} \sin \xi_{j} x\right)
\end{aligned}
$$


where $B_{j}$ is the $n$-th positive root of

$$
\tan 3_{j}{ }^{2}=\frac{h_{j}}{\beta_{j}}
$$

The above equation does not consider radiodecay and can become slightly more cumbersome if pursued. However, note that Danckwerts' method (Danckwerts, 1951) used previously to examine the effect of radiodecay on diffusion transport is not applicable to Equations $(3.5 .49)-(3.5 .52)$ because $c(x, 0)=0$.

In order to examine the diffusion transport with radiodecay to the jeology from the backfill after inventory depletion, the following equations with a fixed boundary condition at $x=0$ rather than a "radiation" boundary condition are examined

$$
\begin{aligned}
& \frac{\partial^{2} c}{\partial z^{2}}-b z-\frac{\partial c}{\partial t}=0 \\
& c(0, t)=0 \\
& \frac{d c}{d z}(1, t)=0 \\
& c(z, 0)=f(z)
\end{aligned}
$$

These equations result in a significantly simpler solution form than the "radiation" case. The solution to the above equations can be obtained by making the substitution

$$
c(z, t)=e^{-b t} u(z, t)
$$

which results in the following

$$
\begin{aligned}
& \frac{\partial^{2} u}{\partial t^{2}}-\frac{\partial u}{\partial t}=0 \\
& u(0, t)=0 \\
& \frac{d u}{d t}(1, t)=0
\end{aligned}
$$

$$
u(z, 3)=f(z)
$$


The solution can be obtained by conventional methods, and for the case when steady state is attained in the backfill, $f(z)$ is Equation $(3.5 .41)$. The solution is

$$
c(z, t)=\frac{2 \sqrt{b} \cosh \sqrt{5}}{\sinh \sqrt{5}} \sum_{n=1}^{\infty} \frac{(-1)^{n+1} \sin \lambda_{n} z}{b+\lambda_{n}^{2}} \exp \left[-\left(b+\lambda_{n}{ }^{2}\right) t\right]
$$

where $\lambda_{n}=(2 n-1) \pi / 2$. That the $t=0$ solution is equal to Equation $(3.5 .41)$ can be verified numerically. Aiso, for $b=0$, the solution at $t=0$ predicts the concentration profile for the initial condition $f(z)=z$.

The radio-diffusion parameter, $b$, appears in Equation (3.5.64) in the same manner as before, i.e., $b+\lambda_{n}^{2}$. For the radionuclides studied in this study, $b \ll 1$ which is justification for ignoring $b$ in the calculated results.

The calculated results for the case of $b=10$ are presented in Figure 3-13. Note that the concentration profile has essentially attained the $\theta=\infty$ value when $\theta<0.1$.

In the event the concentration profile has not attained steady state when inventory depletion occurs, the solution form is somewhat more complicated because $f(z)$ in Equation (3.5.58) takes the form of Equation $(3.5 .40)$. The solution in this case is

$$
\begin{aligned}
c(z, t)=\frac{4}{\pi^{2}} & \sum_{n=1}^{\infty} \frac{\mid 2(-1)^{n+1}}{(2 n-1)^{2}}+(-1)^{n} \sum_{m=1}^{\infty}\left[\frac{1}{(m-n+1 / 2)(m+n-1 / 2)}\right] . \\
& \cdot\left[\frac{b+\lambda_{m}^{2} \exp \left[-\left(b+\lambda_{m}^{2}\right) T_{0}\right]}{b+\lambda_{m}^{2}}\right] \exp \left[-\left(b+i_{n}^{2}\right) t\right] \cdot \\
& \left.\cdot \sin \lambda_{n}{ }^{2}\right\}
\end{aligned}
$$

where $\lambda_{m}=m \pi$ and $T_{0}$ is the time at which zero inventory is attained.

since it appears that essentially zero concentration in the backfill is attained for the dimensionless time $\theta \approx 0.1$, an estimate of "zero" release to the geology can be obtained for the case of americium-241 for a typical backfill. 


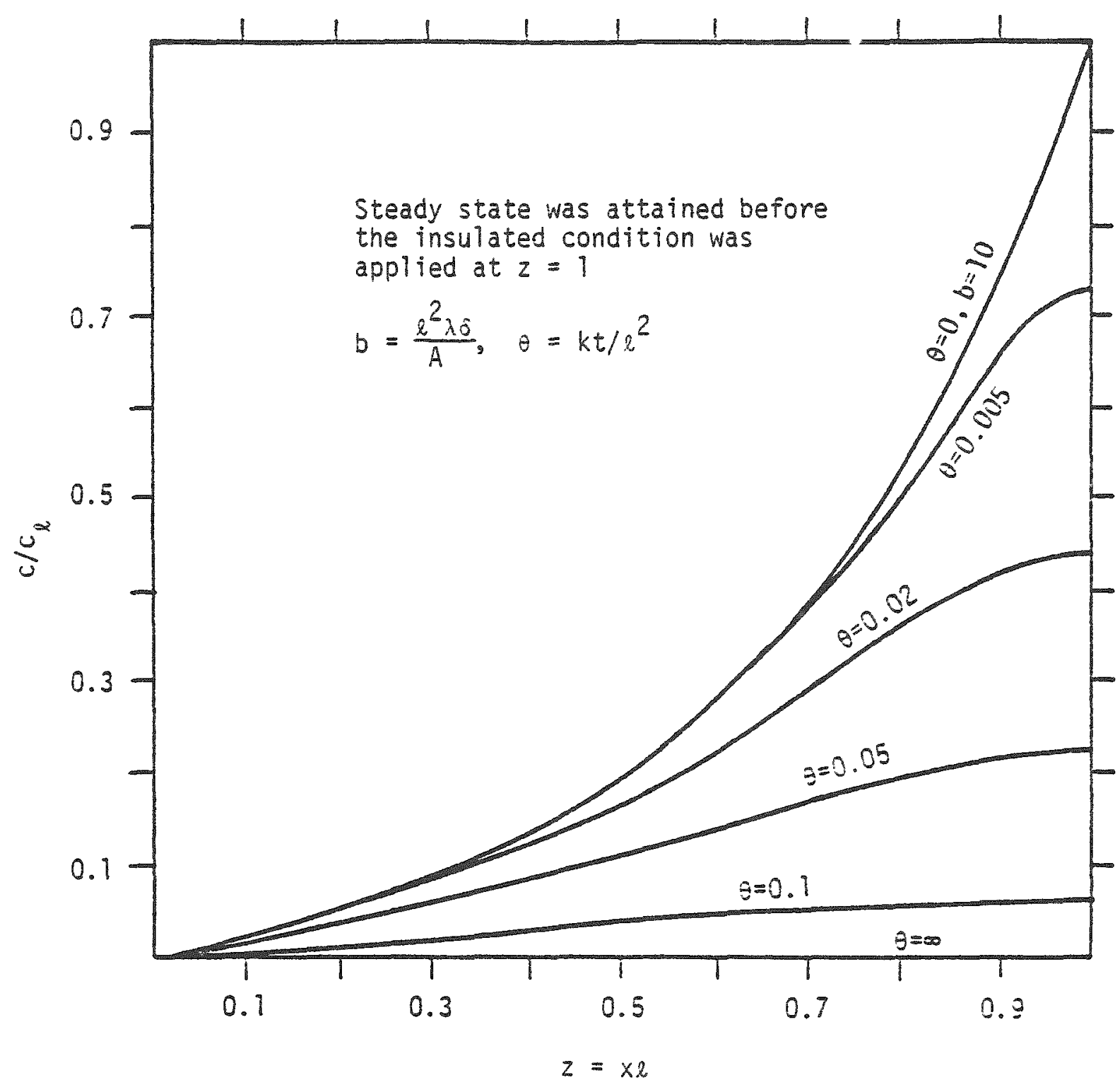

SA:-3CEK-13

Figure 3-13. Concentration Profiles for Zero-inventory Release to Geology with $b=10$. 
The parameters used for americium-241 are $k \approx 3.9 \times 10^{-4} \mathrm{~cm}^{2} / \mathrm{yr}$ and $i \approx 30.51 \mathrm{~cm}$, so that

$$
\approx \approx 0.1=k t / \ell^{2}
$$

or $t \approx 2.5 \times 10^{5}$ years.

A flowchart for the RELEAS subroutine is presented in Figure 3-14. The only input required from the main program are three barrier diameters which are used to calculate $h_{2}$ as defined in Equation (3.5.2). A i isting of RELEAS is provided in Appendix $A$.

\subsection{RADIONUCLIDE RADIATION FIELDS}

The objective of the radiation field subroutine RADCLC is to calculate the radiation exposure from gamma rays at the outer surface of each package barrier as a function of time after package emplacement. The radiation source in the model is assumed to be PWR spent fuel with a burnup of 33,000 MNd/MT. The emplacement time is assumed to be 6.5 years after discharge and the burnup is assumed to be constant over 1100 days. The fuel composition assumed 13.3 percent enriched) is given in Table 3-7.

Various materials are chosen for use in the engineered barriers of a package design. Compositions and densities of some of these materials are obtained to estimate the radiation attenuation characteristics. The data for the other materiais are estimated or assumed. The following paragraphs identify the compositions used in the radiation analysis.

\section{Bentonite}

Bentonite is a naturally occurring clay characterized by the faci that it swells upon absorbing water. The main component is montmorillonite. The chemical composition is

Species Weight Parcent

$\begin{array}{ll}\mathrm{SiO}_{2} & 63.0 \\ \mathrm{Al}_{2} \mathrm{O}_{3} & 21.0\end{array}$




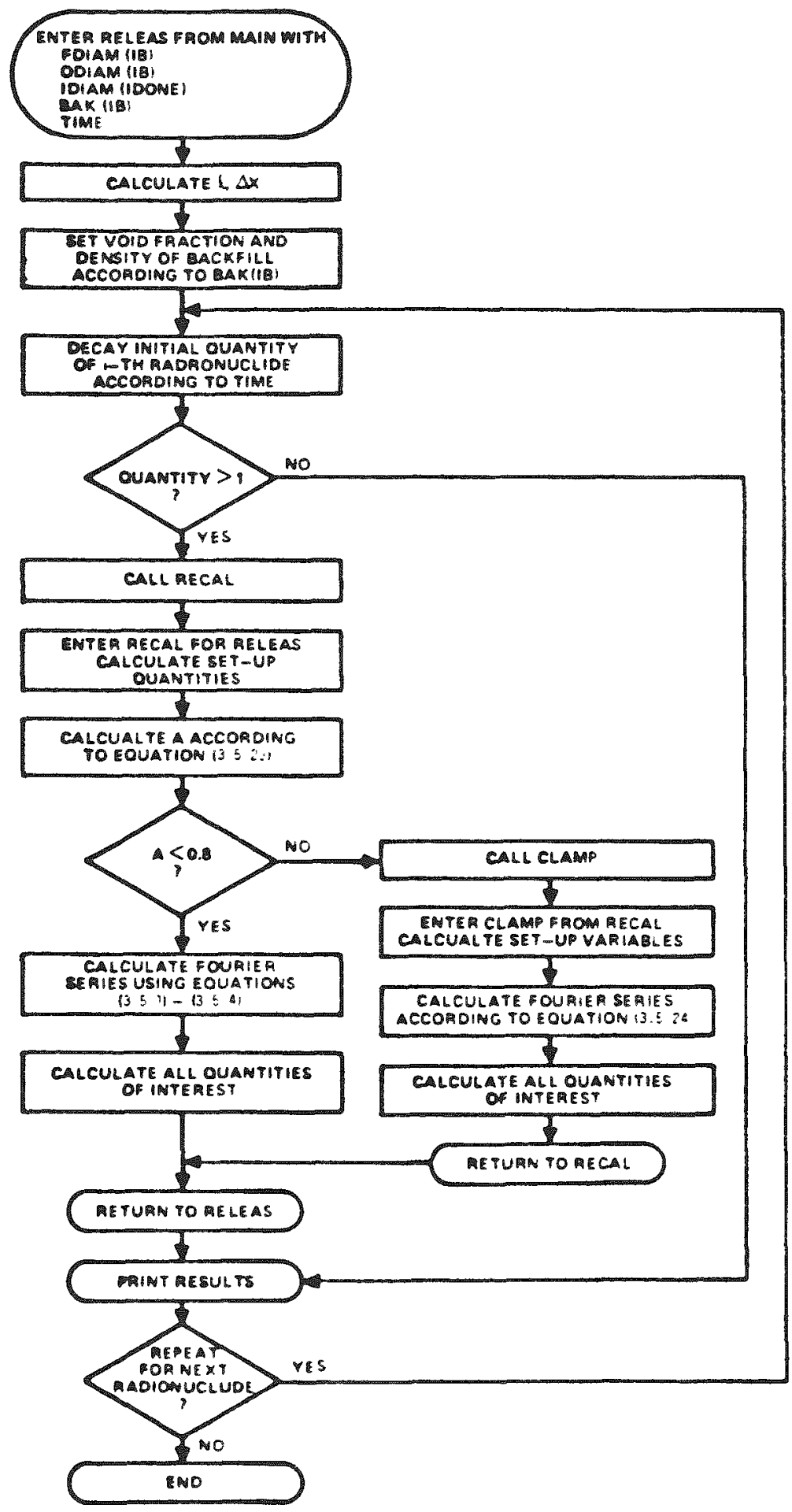

Figure 3-14. RELEAS Flowchart. 
Table 3-7. Fuel Composition.

\begin{tabular}{|c|c|c|c|}
\hline Isotope & Gram-Atoms/MT & Isotope & Gram-Atoms/MT \\
\hline$c^{12}$ & 1.5 & $2 r^{91}$ & 306.725 \\
\hline$A 7^{27}$ & 4.0 & $2 r^{92}$ & 462.239 \\
\hline$s i^{28}$ & .607 & $z r^{94}$ & 460.074 \\
\hline$S i^{29}$ & .034 & $2 r^{96}$ & 72.50 \\
\hline$T i^{46}$ & .304 & $\mathrm{Nb}^{93}$ & 10.258 \\
\hline$T i^{47}$ & .277 & $M_{0}^{92}$ & .957 \\
\hline $\mathrm{Ti}^{48}$ & 2.771 & $\mathrm{Mo}^{94}$ & .532 \\
\hline$T i^{49}$ & .204 & $M^{95}$ & .926 \\
\hline $\mathrm{Ti}^{50}$ & .200 & $M_{0}{ }^{96}$ & .958 \\
\hline$c r^{50}$ & 5.040 & $\mathrm{Mo}^{97}$ & .546 \\
\hline$C r^{52}$ & 57.423 & $\mathrm{Mo}^{98}$ & 1.357 \\
\hline$c r^{53}$ & 6.415 & $\mathrm{MO}^{100}$ & .540 \\
\hline$c r^{54}$ & 1.574 & $S n^{112}$ & .321 \\
\hline $\mathrm{Mn}^{55}$ & .327 & $S n^{114}$ & .219 \\
\hline $\mathrm{Fe}^{54}$ & 4.037 & $S n^{115}$ & .113 \\
\hline $\mathrm{Fe}^{56}$ & 61.018 & $s n^{116}$ & 4.681 \\
\hline $\mathrm{Fe}^{57}$ & 1.439 & $s n^{117}$ & 2.470 \\
\hline $\mathrm{Fe}^{58}$ & .310 & $\mathrm{Sn}^{118}$ & 7.729 \\
\hline $\mathrm{Co}^{59}$ & .915 & $S n^{119}$ & 2.739 \\
\hline$N i^{58}$ & 111.862 & $\mathrm{Sn}^{120}$ & 10.392 \\
\hline $\mathrm{Ni}^{60}$ & 47.783 & $\mathrm{Sn}^{122}$ & 1.467 \\
\hline$N i^{61}$ & 1.869 & $s n^{124}$ & 1.323 \\
\hline $\mathrm{Ni}^{62}$ & 5.645 & $u^{234}$ & 1.13 \\
\hline $\mathrm{Ni}^{64}$ & 1.609 & $u^{235}$ & 140.1 \\
\hline $2 r^{90}$ & 1421.122 & $u^{238}$ & 4062.0 \\
\hline
\end{tabular}




$\begin{array}{ll}\mathrm{Fe}_{2} \mathrm{O}_{3} & 3.2 \\ \mathrm{FeO}^{2} & 0.3 \\ \mathrm{TiO}_{2} & 0.1 \\ \mathrm{CaO}^{2} & 0.7 \\ \mathrm{MgO} & 2.7 \\ \mathrm{Na}_{2} \mathrm{O} & 2.2 \\ \mathrm{~K}_{2} \mathrm{O} & 0.4 \\ \mathrm{H}_{2} \mathrm{O} & 5.6 \\ \text { Other } & 0.8 \\ & \\ \text { Density }-2.1 \mathrm{gm} / \mathrm{cm}^{3}\end{array}$

$\underline{\text { Sand }}$

Sand is assumed to be 100 percent $\mathrm{SiO}_{2}$ with a density of $2.1 \mathrm{gm} / \mathrm{cm}^{3}$.

Clinoptilolite

clinoptilolite is assumed to be a clay with a density of $2.2 \mathrm{gm} / \mathrm{cm}^{3}$.

Mild Steel

Mild steel is assumed to be 100 percent $F e$ with a density of $7.85 \mathrm{gm} / \mathrm{cm}^{3}$.

Zircaloy-2

The composition and density used for Zircaloy-2 is

$\begin{array}{cc}\text { Element } & \text { Neight Percent } \\ \mathrm{Zr} & 38.24 \\ \mathrm{Cr} & 0.1 \\ \mathrm{Fe} & 0.21 \\ \mathrm{Sn} & 1.45\end{array}$




$$
\text { Density }=6.55 \mathrm{gm} / \mathrm{cm}^{3}
$$

Inconel -600

The composition and density used for Inconel-600 is

\begin{tabular}{lc} 
Element & Weight Percent \\
\cline { 2 - 2 }$N$ i & 75.41 \\
$\mathrm{Cr}$ & 15.5 \\
$\mathrm{C}$ & .08 \\
$\mathrm{Si}$ & .25 \\
$\mathrm{Fe}$ & 8.0 \\
$\mathrm{Ca}$ & .25 \\
Mn & .50 \\
& \\
Density & $-8.43 \mathrm{gm} / \mathrm{cm}^{3}$
\end{tabular}

SST-304

The composition and density used for 304 Stainless Steel is

Element Weight Percent

$\begin{array}{lr}\mathrm{Fe} & 68.17 \\ \mathrm{Cr} & 18.94 \\ \mathrm{Ni} & 10.51 \\ \mathrm{Mn} & 1.75 \\ \mathrm{Si} & .53 \\ \mathrm{C} & .06\end{array}$

Density $-7.93 \mathrm{gm} / \mathrm{cm}^{3}$ 
Copper

The density used for copper is $8.92 \mathrm{gm} / \mathrm{cm}^{3}$.

Lead

The density used for lead is $11.34 \mathrm{gm} / \mathrm{cm}^{3}$.

Cast Iron

$7.85 \mathrm{gm} / \mathrm{cm}^{3}$.

Cast iron is assumed to be 100 percent Fe with a density of

Helium

The effect of helium as a shielding material is neglected and assumed to be void.

Air

The effect of air as a shielding material is neglected and assumed to be void.

An ORIGEN calculation is performed to obtain the radiation source term for the fuel bundle. In order to confirm that the radiation source term used in the code is consistent with the data in (DOE, 1979), a comparison was made with the radioactivity content and heat generation rate in spent fuel as presented in Table 5.7.2 of that document. This comparison is shown in Table 3-8. Nith the exception of tritium and $c^{14}$ the calculations in this study agreed with the referenced data. It is concluded that the radiation source term used is consistent with the previous data.

The photon release rate versus tine after emplacement is shown in Figure 3-15 for both gamma rays from fission products and from activation products. The photon spectra from fission products and activation products are shown in Figures $3-15$ and $3-17$, respectively. The photon spectra are shown as photon/sec/iev nomalized to one photon and are shown for one year and 100 years after emplacement. The photon soectra from fission products is seen to be 
Table 3-8. Comparison of Radioactivity Content and Heat Generation in Spent Fuel with Prior Data.

\begin{tabular}{|c|c|c|}
\hline & \multicolumn{2}{|c|}{$\begin{array}{l}\text { Fission Product Content } 6.5 \\
\text { Years after Discharge, } \mathrm{Ci}\end{array}$} \\
\hline & Table 5.7.2* & This Work \\
\hline \multicolumn{3}{|l|}{ Fission Product } \\
\hline$H^{3}$ & $3.1 \times 10^{2}$ & $5.7 \times 10^{-5}$ \\
\hline $\mathrm{Kr}^{85}$ & $6.5 \times 10^{3}$ & $6.3 \times 10^{3}$ \\
\hline$I^{129}$ & $3.3 \times 10^{-2}$ & $3.3 \times 10^{-2}$ \\
\hline $5 r^{90}+\gamma^{90}$ & $1.2 \times 10^{5}$ & $1.3 \times 10^{5}$ \\
\hline$Z r^{95}+N b^{95}$ & $3.9 \times 10^{-5}$ & $5.4 \times 10^{-5}$ \\
\hline$R u^{106}+R h^{106}$ & $1.1 \times 10^{4}$ & $1.2 \times 10^{4}$ \\
\hline $\mathrm{Cs}^{134}+\mathrm{Cs}^{137}+\mathrm{Ba}^{137}$ & $1.8 \times 10^{5}$ & $2.1 \times 10^{5}$ \\
\hline$C e^{144}+\operatorname{Pr}^{144}$ & $5.8 \times 10^{3}$ & $6.7 \times 10^{3}$ \\
\hline \multicolumn{3}{|l|}{ Actinide } \\
\hline $\mathrm{Pu}^{239}$ & $2.9 \times 10^{2}$ & $3.2 \times 10^{2}$ \\
\hline$P_{u}^{241}$ & $8.4 \times 10^{4}$ & $7.7 \times 10^{4}$ \\
\hline $\mathrm{Cm}^{242}+\mathrm{Cm}^{244}$ & $1.0 \times 10^{3}$ & $1.9 \times 10^{3}$ \\
\hline \multicolumn{3}{|l|}{ Activation Product } \\
\hline $\mathrm{Fe}^{55}$ & $1.0 \times 10^{3}$ & $3.5 \times 10^{2}$ \\
\hline $\mathrm{Co}^{60}$ & $2.1 \times 10^{3}$ & $2.7 \times 10^{3}$ \\
\hline$Z r^{95}+N b^{95}$ & $9.0 \times 10^{-7}$ & $9.0 \times 10^{-7}$ \\
\hline Heat Generation Rate, W/MTHM & $1.4 \times 10^{3}$ & $1.6 \times 10^{3}$ \\
\hline
\end{tabular}

*Taken from (DOE, 1979) 


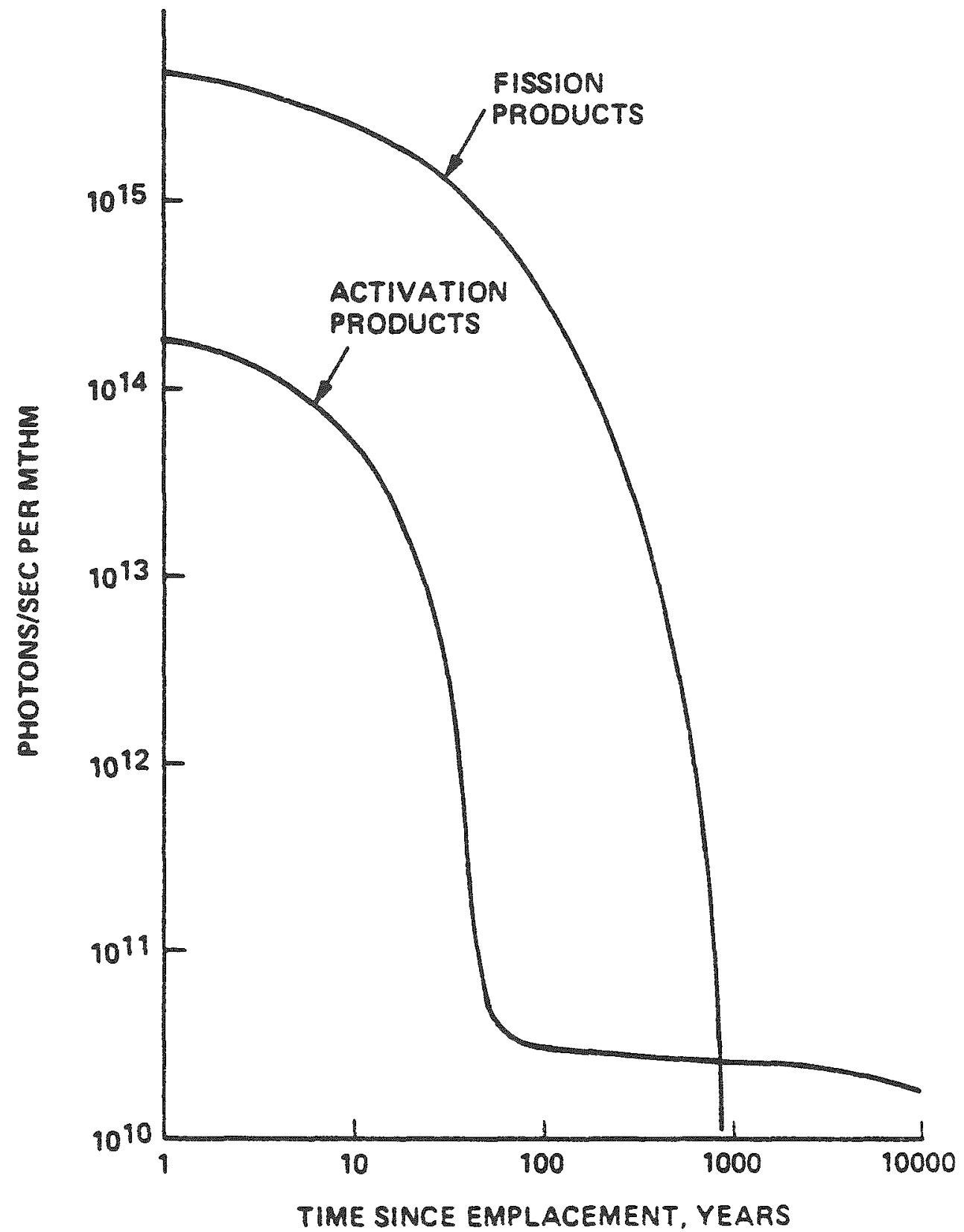

Figure 3-15. Photon Release Rate Vs Time After Emplacement. 


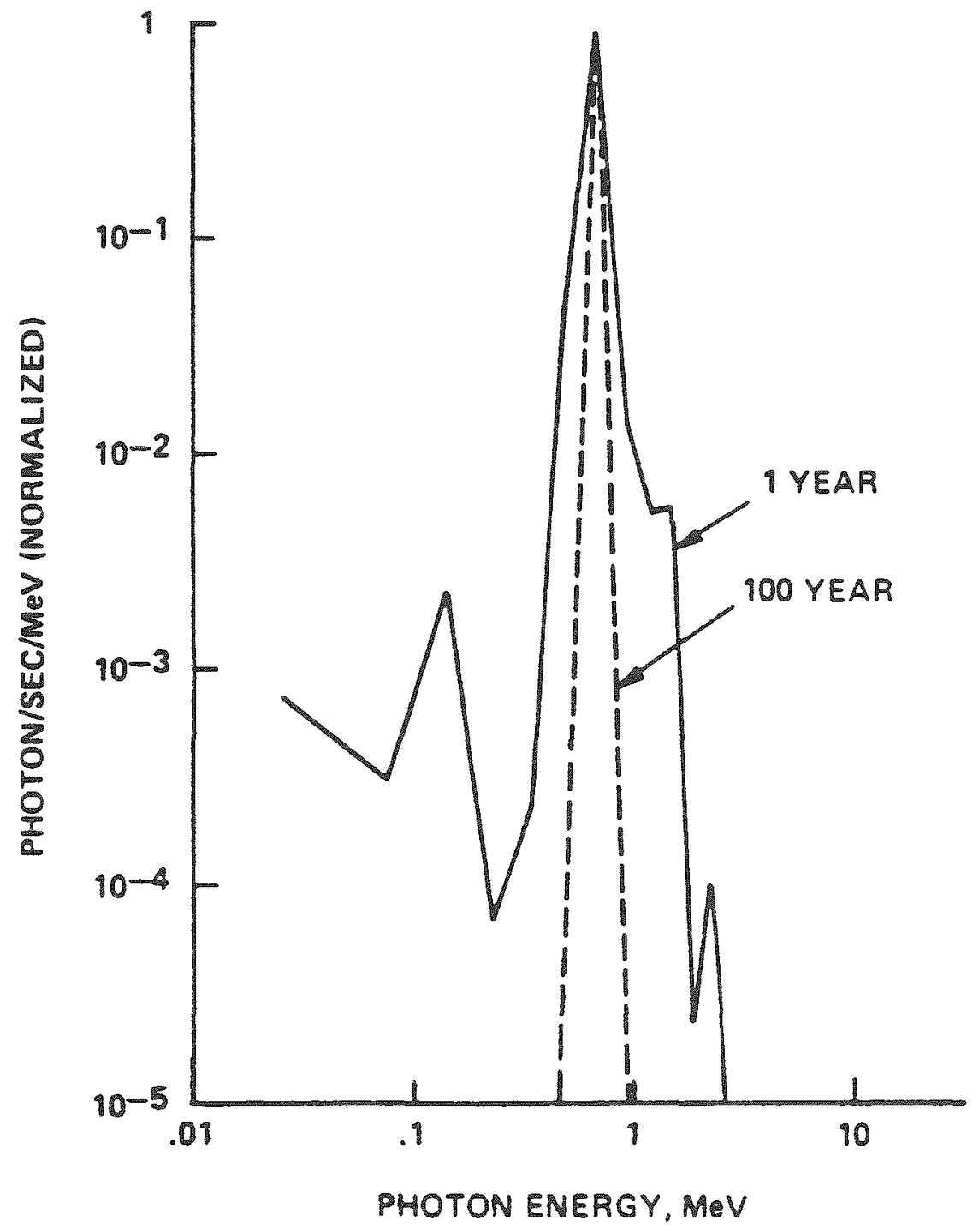

Figure 3-16. Photon Spectrum from Fission Products. 


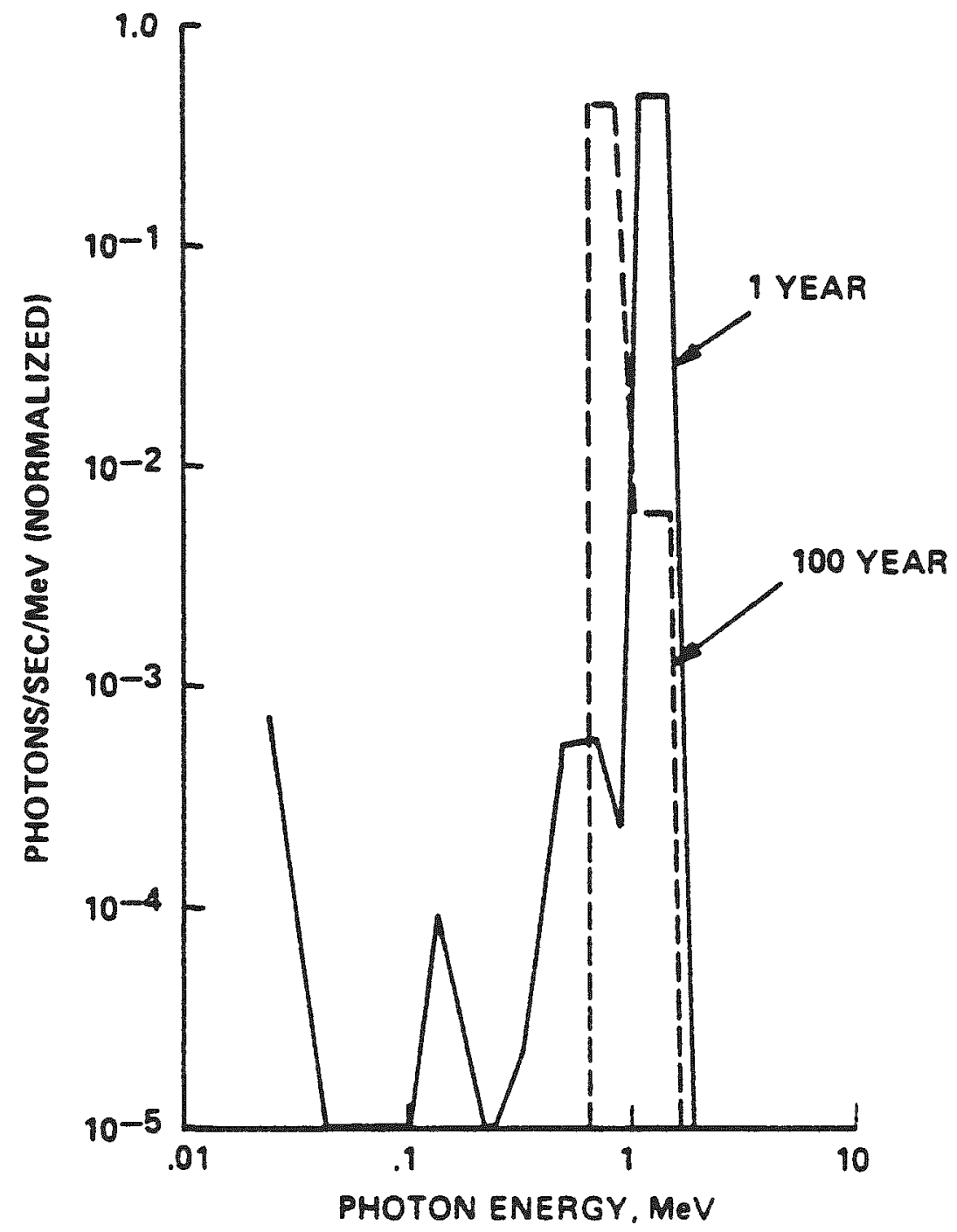

Figure 3-17. Photon Spectrum from Activation Products. 
strongly peaked about $0.7 \mathrm{MeV}$ and relatively insensitive to decay time. The spectra for times greater than 100 years are essentialiy unchanged from 100 years. The photon spectra from activation products shown in Figure 3-17 indicate that there is some time dependence to the spectra. For short times (one to ten years) the spectrum peaks at slightly greater than 1 MeV. However, for time greater than 100 years, the spectrum has softened to about $0.7 \mathrm{MeV}$. From Figure 3-17, it can be seen that the photons from activation products become important only after about 1000 years and longer. Therefore, the pnoton spectra for both fission products and activation products are assumed to be about $0.7 \mathrm{MeV}$ since during the time regine that each component is important, the spectra are strongly peaked about 0.7 Mev.

It is fortunate that the photon spectrum is roughly monoenergetic since it allows the use of a monoenergetic cross-section. Table 3-9 lists the linear attenuation coefficient for the materials considered in this study.

The calculation of the gamma ray flux at a particular location in the package employs a simple equation (Rockwell, 1956) for the flux from a cylindrical source

$$
F \text { lux }=B \times s_{v} \times \frac{R_{0}^{2}}{a+z} \times F\left(b_{2}\right)
$$

where

$$
\begin{aligned}
B= & \text { buildup factor (dimensionless) } \\
S_{V}= & \text { source intensity, (photons } \left./ \mathrm{cm}^{3} / \mathrm{sec}\right) \\
R_{0}= & \text { radius of the cylinder, }(\mathrm{cm}) \\
a= & \text { distance to the point of interest from the edge } \\
& \text { of the cylinder, (cm) } \\
z= & \text { self-shielding distance factor, }(\mathrm{cm}) \\
b_{2}= & \text { number of mean free paths to the point of } \\
& \text { interest, (dimensionless) } \\
F= & \text { function defined by }
\end{aligned}
$$

$$
F\left(b_{2}\right)=\int_{0}^{\pi / 2} e^{-b_{2} \sec s} d e
$$


Table 3-9. Linear Attenuation Coefficients for Materials

\begin{tabular}{|l|c|c|}
\hline Material & Density, $\mathrm{gm} / \mathrm{cm}^{3}$ & Attenuation Coefficient, $\mathrm{cm}^{-1}$ \\
\hline Backfill Material & & .130 \\
Bentonite & 2.1 & .140 \\
Sand \& Bentonite & 2.1 & .130 \\
Clinoptilolite & 2.2 & \\
Other Materials & & \\
Mild Steel & 7.85 & .470 \\
Zircaloy-2 & 6.55 & .367 \\
Inconel-600 & 8.43 & .472 \\
SS-304 & 7.93 & .462 \\
Copper & 8.92 & .500 \\
Lead & 11.34 & .797 \\
Cast Iron & 7.85 & .470 \\
Helium & - & $8 \times 10^{-5}$ \\
Air & - & $8 \times 10^{-5}$ \\
\hline
\end{tabular}


Suildup data for various materials are available in the form of a paraneter fit to the equation (Rockwel1, 1956)

$$
3\left(a_{1} b_{2}\right)=A_{1} e^{-a_{1} b_{2}}-A_{2} e^{-a_{2} b_{2}}
$$

where

$$
A_{1}, A_{2}, a_{1} \text {, and } a_{2} \text { are a function of energy. }
$$

The values for the buildup factor parameters evaluated at 0.7 lev are shown in Table 3-10. Although buildup factors were not available for all of the specific materials of interest in this work, buildup factors were reasonably approximated by those that were available. Table 3.11 gives the correspondence between the barrier materials and the buildup material used in the analysis.

The RADCLC subroutine was written implementing the procedures described. The output of the subroutine, DOSE, has the units of $\mathrm{R} / \mathrm{hr}$.

A flowchart for RADCLC is shown in Figure $3-18$ and a progran listing is provided in Appendix A.

\subsection{WATER REPELLENT BACKFILLS}

The model assumes that at time zero the backfill is saturated with water and that water-induced degradation processes proceed from that point. Furthemore, the possible attentuating effects on corposion of reduced water and/or solute transport through the backfill are not considered. Corrosion is assumed to proceed as if the material is immersed in the water. The action of the backfill to reduce or eliminate water flow to (or from) the package is not considered mainly because there is available no basis on which to evaluate the functional life. This section presents a discussion on the zossibie effects such a backfill could have on the results of nodel calculations.

Two modes of backfill behavior would be beneficial in the period prior to package failure

- total exclusion of water from the outer wall of the multiple barsier systen for sone period of time 
Table 3-10. Buildup Factor Parameters at $0.7 \mathrm{MeV}$.

\begin{tabular}{|l|c|c|c|c|}
\hline \multirow{2}{*}{ Material } & \multicolumn{4}{|c|}{ Parameter Values } \\
\cline { 2 - 5 } & $A_{1}$ & $A_{2}$ & $a_{1}$ & $a_{2}$ \\
\hline Iron & 9 & -8 & -.081 & .0255 \\
Lead & 2.3 & -1.3 & -.04 & .17 \\
Concrete & 10 & -9 & -.088 & .03 \\
Water & 11 & -10 & -.104 & .03 \\
\hline
\end{tabular}


Table-3-11. Buildup Factor Material Correspondence.

\begin{tabular}{|l|c|}
\hline Material & Buildup Material \\
\hline Bentonite & Concrete \\
Sand & Concrete \\
Clinoptilolite & Concrete \\
Mild Steel & Iron \\
Zircaloy-2 & Iron \\
Inconel-600 & Iron \\
SS-304 & Iron \\
Copper & Lead \\
Lead & Lead \\
Cast Iron & Iron \\
Helium & None, $B=1$ \\
Air & None, $B=1$ \\
\hline
\end{tabular}




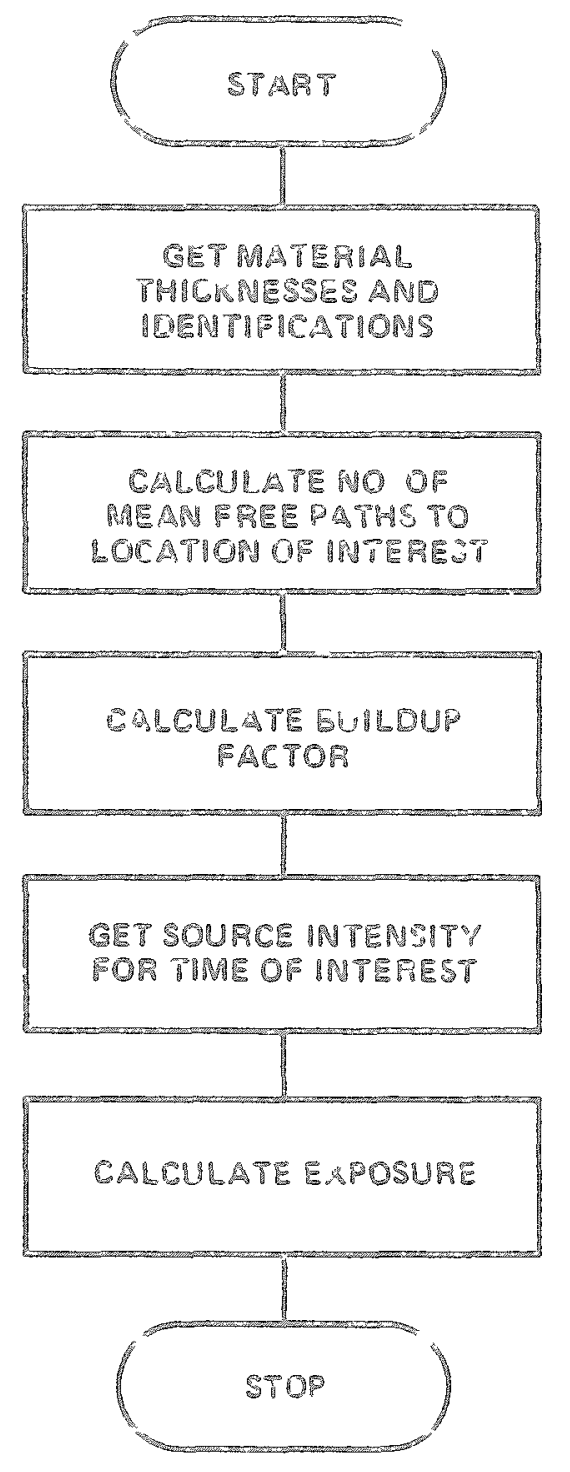

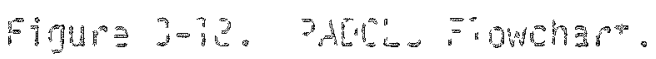


- high resistabe io water and soluce brastort through bhe baction

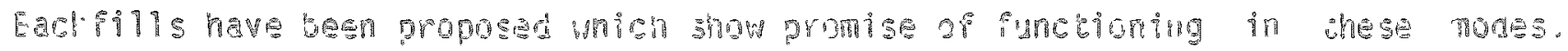

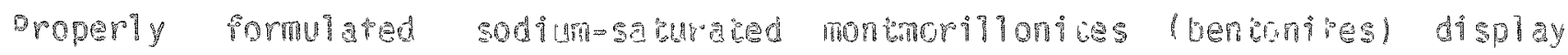

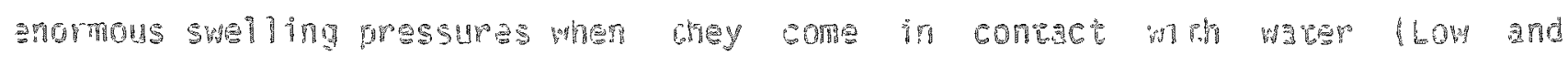

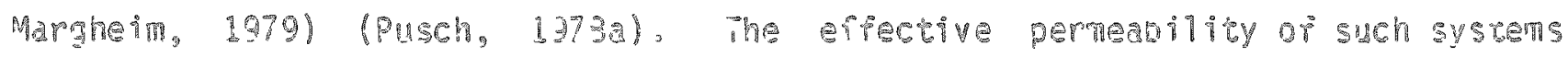
beones ssentially zero as characterized in Taole 3-12. The sweling phenomenon includes bhe abilicy to seli-seal and also bo seal crachs in the adjacenc pol (Pusch, 1970b). Juch a material cuth function as a total exclusion barpier for sone bine. The key question is hou $1 \mathrm{mng}$ such a material will retain ibs

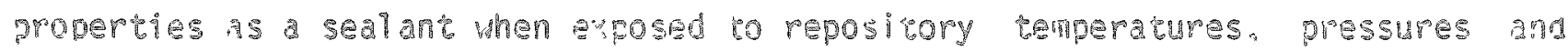
possivly comrosive water. Evidence on longorem benavior is iacking bur

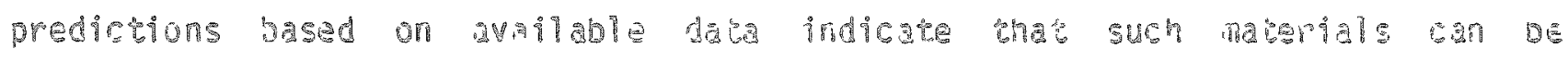
stabilized for example. use of quartz stabilizes monmontitonite and reduces the

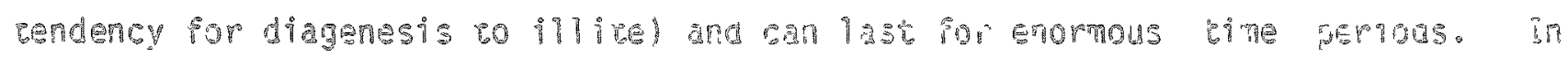
such a case, pure delay bime would be incroduced into bre pesulgs prom the 3ARIER code. This is because there is no mechanism or degradaion withour uder

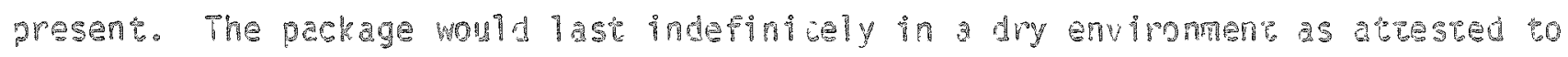
by many ancient zrotracts discovered by archeologists in such environments.

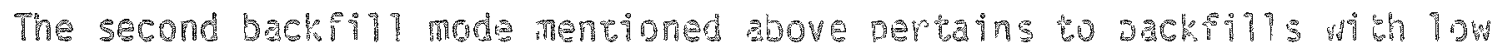
permeability. In this ase water and solute transpoptore greaty innibibed over an extended period of time. Studies indicace that degradarion pares wout ae

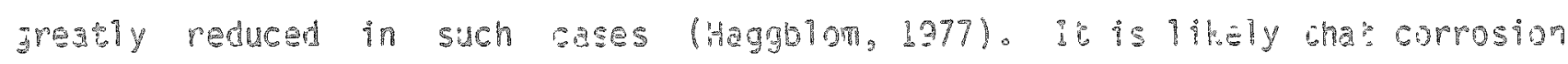
rates used in BARER gere somewnat high and that a paduced sorrosion bace would be ooserved as long as the backitil prained intar. Such effecbs would raure further study if it became desirable to account for them in the perfomance evaluation.

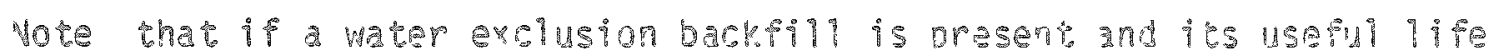

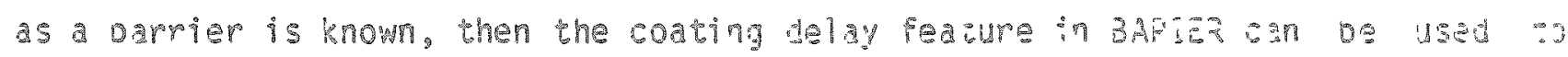

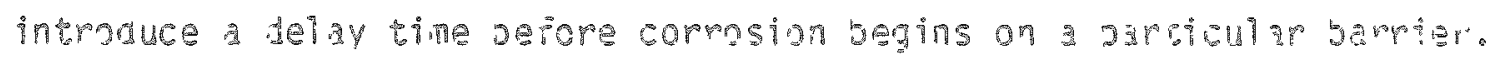


Table 3-12. Permeability of Clays and Sand-Clay Mixtures (Ende11, 1938).

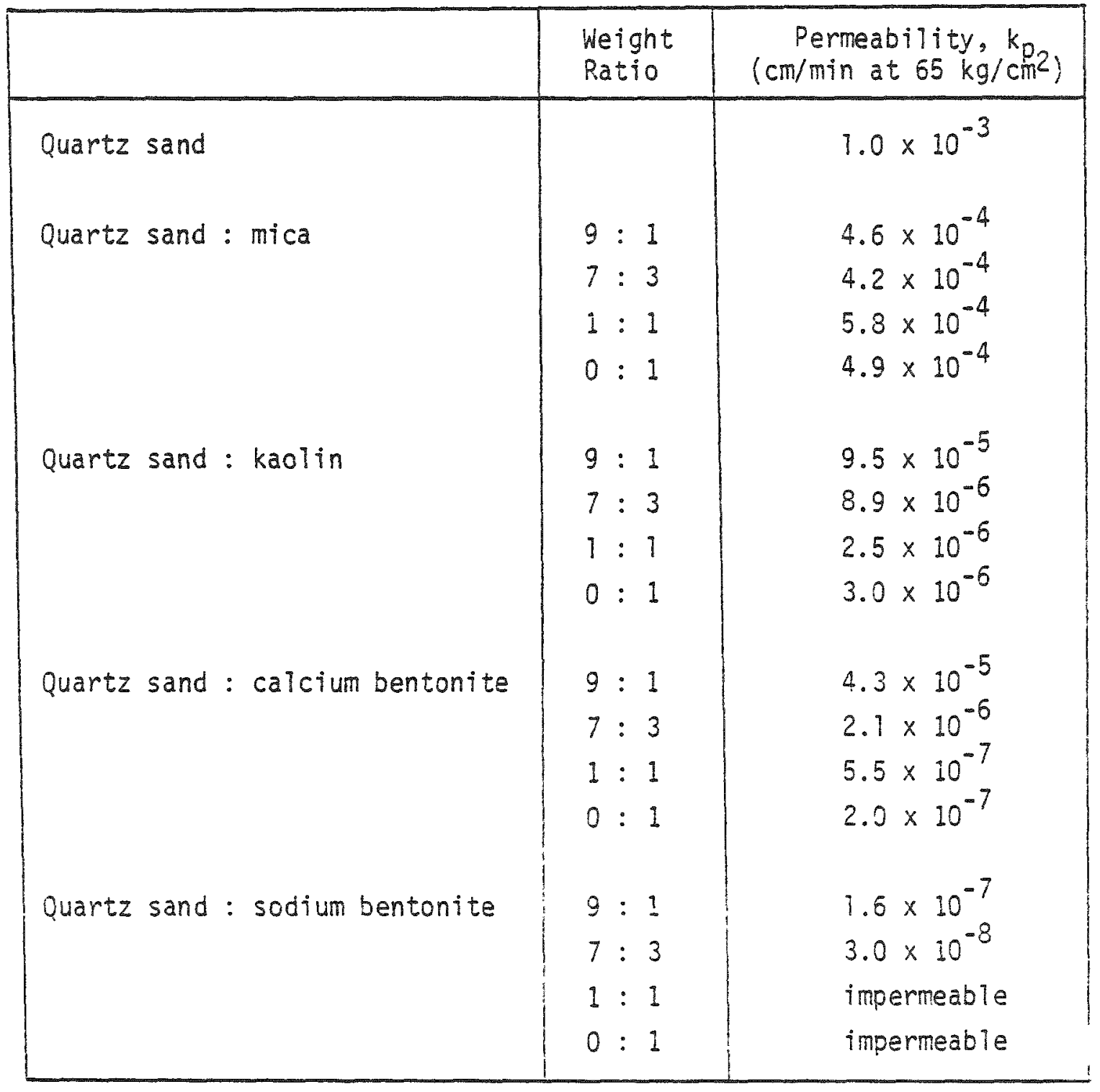




\section{PACKAGE DESIGN DESCRIPTIONS}

The package designs evaluated in this study were a subset of a series of designs studied in previous work (Stula, 1980a) (Lester, 1979) with some additional refinements. The designs were restricted to the basic concepts which were described in the SURF program (Westerman, 1979). The philosophy used to choose the design for this study was to pick promising concepts from previous studies which gave a representative spectrum of behavior, complexity and probable cost. Thus, the purposes of the analysis was to provide a basis for programatic planning and not an optimization of designs or search for the "best" designs.

\subsection{PREVIOUS WORK}

A large selection of design possibilities was studied in past work using a more simplified version of the "BARIER" code. Four basic SURF program concepts were considered as shown in Figure $4-1$ and designated $A, B, C, D$. An additional concept was studied in which the stabilizer was a cast-in-place solid rather than segmented blocks. This was designated concept "E". Variation on each of the concepts (i.e., different dimensions and materiais) are designated A.1, A.2, or B.1, etc. Table $4-1$ is a table of concepts studied in the first series of evaluations in previous work (Lester, 1979) and Table 4-2 is a subsequent series from follow-on studies using the previous version of 3ARIER (Stula, 1980a).

The results of the past studies yielded a list of concepts which were the best in performance in each of the major concept categories. These conceots and the perfomance results from the old version of BARIER are summarized in Table 4-3. These concepts were reevaluated in this study using the new version of BARIER and the results are given in this report.

The package designs used in the current study are described in the following sections and sumarized in Table $4-4$. 
The cast stabilizer "E" concept showed promising results in previous work when the geology was a high creep medium (i.e., salt or shale). in effect, the cast stabilizer and the canister act like a solid rod in resisting nedium crushing forces. Included in the current group of Concept $\Xi$ cases were concepts E.3, E.4, and E.24, which were analyzed with the old version of BARIER. Hew variations on this concept in this study were designated with an "y" suffix and included changes in materials and material thickness.

\subsection{HEAVY SLEEVE PACKAGES (CONcept B1)}

Another promising concept from past work was the "BI" design which incopporates a heavy-walled sleeve to line the bore-hole. The sleeve serves as a defense against high crushing forces in a creeping medium. The stabilizer in this design is a segmented solid or a gas filler. The only other barpier element is the canister which contributes very little to long-tem defense. Concepts 31.7 and 31.11 were promising designs in the former evaluation using the old version of BARIER. These were reevaluated with the new nodel. In addition, severai variations are introduced as suffix "n" cases such as $31.1 \mathrm{M}$. These package design variations are summarized in Table $4-4$.

\subsection{COMBPNATION SLEEVE/CAST STABILIZERS (CONCEPT 3E) \\ This type of package was a new design analyzed in this study.} Designated as "BE" it combines the heavy sleeve and cast stabilizer features. In a creeping medium this package offers a redundant defense against the medium crushing forces. The package design variations studied are summarized in Table 4-4. 


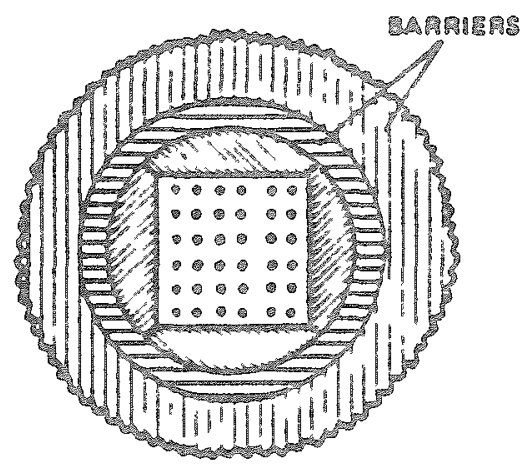

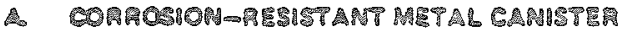

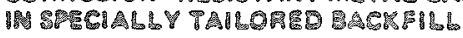

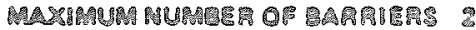

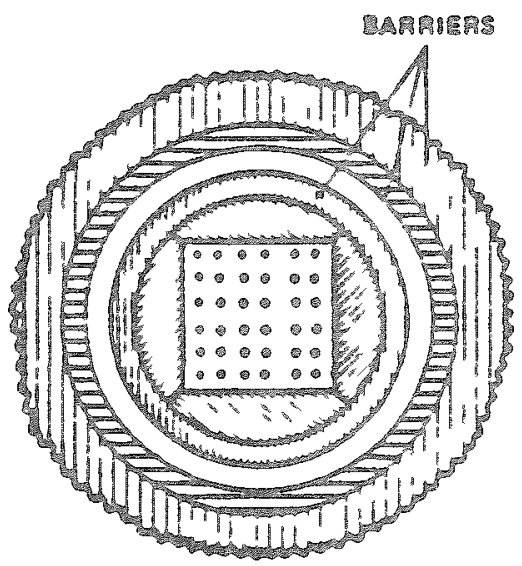

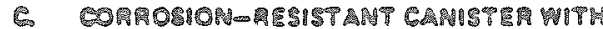

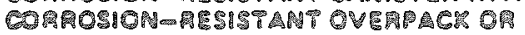

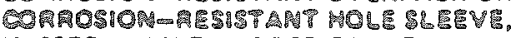

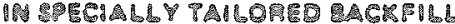

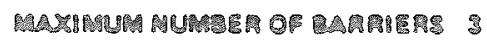

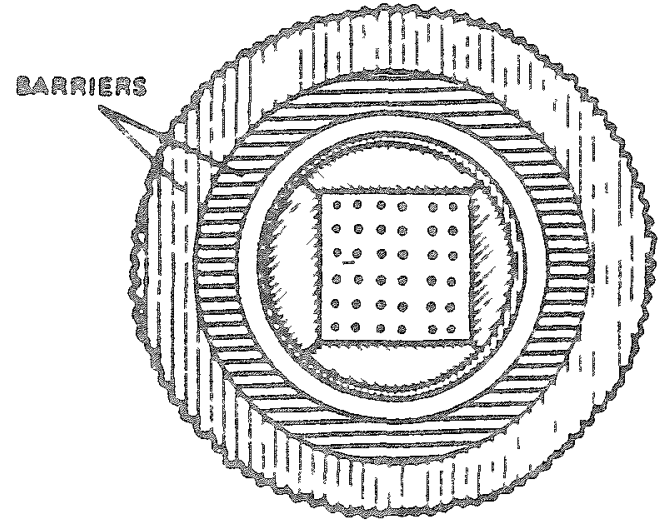

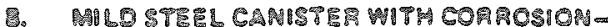

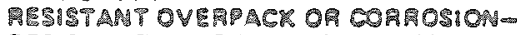

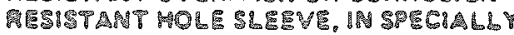

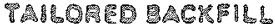

Ma:M

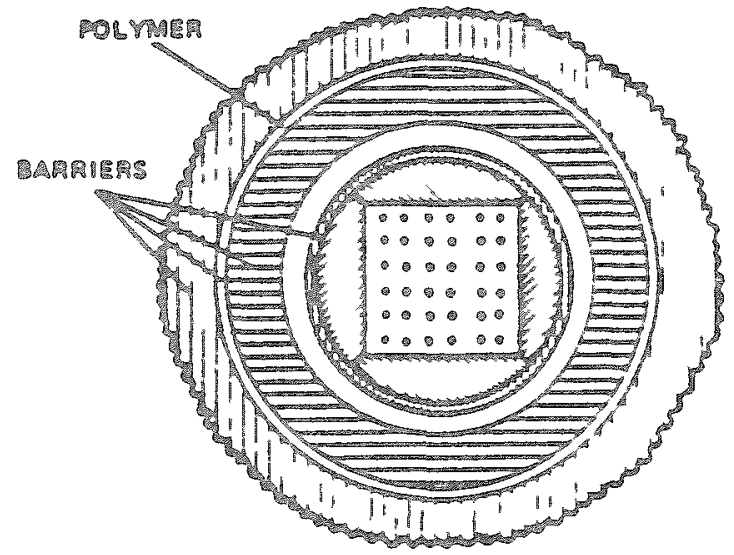

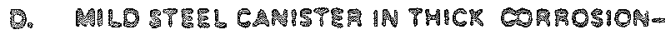
RRESISTMNT EOAR SLPEVE WITM SUAROUNOING

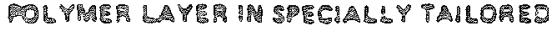
ackerats

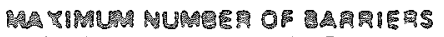

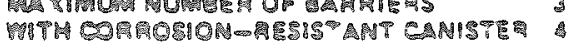

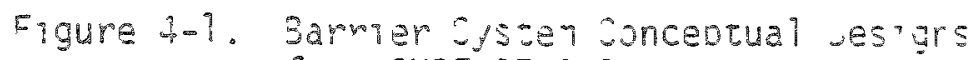
from SUPF SFHP Drogram.
} 
Table 4-1. Summary of Concepts Studied in FY'79 with

Previous Version of "BARIER" Code. (Lester, 1979).

\begin{tabular}{|c|c|c|c|c|c|c|c|c|c|c|}
\hline \multirow[t]{2}{*}{ Concept } & \multirow[t]{2}{*}{ Element } & \multicolumn{2}{|c|}{$\begin{array}{l}\text { Inside } \\
\text { Material }\end{array}$} & \multicolumn{2}{|c|}{$\begin{array}{l}\text { Outside } \\
\text { Material }\end{array}$} & \multirow{2}{*}{$\begin{array}{c}\text { Filler } \\
00 \\
\end{array}$} & \multirow[t]{2}{*}{$\begin{array}{c}\text { Inside } \\
\text { Material }\end{array}$} & \multirow[t]{2}{*}{$\begin{array}{l}\text { Outside } \\
\text { Material }\end{array}$} & \multirow{2}{*}{$\begin{array}{c}\text { Coating } \\
\text { oelay } \\
\text { (yrs) }\end{array}$} & \multirow[t]{2}{*}{ Filler } \\
\hline & & 10 & 00 & ID & 100 & & & & & \\
\hline A.1 & $\begin{array}{l}\text { stab } \\
\text { can }\end{array}$ & $\begin{array}{l}12.0 \\
13.5\end{array}$ & $\begin{array}{l}13.5 \\
14.0\end{array}$ & $\begin{array}{l}13.5 \\
14.0\end{array}$ & $\begin{array}{l}13.5 \\
14.0\end{array}$ & $\begin{array}{l}13.5 \\
40.0\end{array}$ & $\begin{array}{l}304 s s t \\
304 s s t\end{array}$ & $\begin{array}{l}3045 s t \\
3045 s t\end{array}$ & $\begin{array}{l}0.0 \\
0.0\end{array}$ & $\begin{array}{l}\text { none } \\
\text { sand-b }\end{array}$ \\
\hline$A: 2$ & $\begin{array}{l}\text { stab } \\
\operatorname{can}\end{array}$ & $\begin{array}{l}12.0 \\
13.5\end{array}$ & $\begin{array}{l}13.5 \\
15.5\end{array}$ & $\begin{array}{l}13.5 \\
15.5\end{array}$ & $\begin{array}{l}13.5 \\
15.5\end{array}$ & $\begin{array}{l}13.5 \\
40.0\end{array}$ & $\begin{array}{l}304 s s t \\
304 s s t\end{array}$ & $\begin{array}{l}304 s s t \\
304 s s t\end{array}$ & $\begin{array}{l}0.0 \\
0.0\end{array}$ & $\begin{array}{l}\text { none } \\
\text { sand-b }\end{array}$ \\
\hline A.3 & $\begin{array}{l}\text { stab } \\
\operatorname{can}\end{array}$ & $\begin{array}{l}12.0 \\
13.5\end{array}$ & $\begin{array}{l}13.5 \\
14.0\end{array}$ & $\begin{array}{l}13.5 \\
14.0\end{array}$ & $\begin{array}{l}13.5 \\
14.0\end{array}$ & $\begin{array}{l}13.5 \\
20.0\end{array}$ & $\begin{array}{l}304 s s t \\
304 s s t\end{array}$ & $\begin{array}{l}304 s s t \\
304 s s t\end{array}$ & 0.01 & $\begin{array}{l}\text { none } \\
\text { sand-b }\end{array}$ \\
\hline A.4 & $\begin{array}{l}\operatorname{stab} \\
\operatorname{can}\end{array}$ & $\begin{array}{l}12.0 \\
13.5\end{array}$ & $\begin{array}{l}13.5 \\
15.5\end{array}$ & $\begin{array}{l}13.5 \\
15.5\end{array}$ & $\begin{array}{l}13.5 \\
15.5\end{array}$ & $\begin{array}{l}13.5 \\
20.0\end{array}$ & $\begin{array}{l}304 s s t \\
304 s s t\end{array}$ & $\begin{array}{l}3045 s t \\
3045 s t\end{array}$ & $\begin{array}{l}0.0 \\
0.0\end{array}$ & $\begin{array}{l}\text { none } \\
\text { sand-b }\end{array}$ \\
\hline A.5 & $\begin{array}{l}\text { stab } \\
\operatorname{can}\end{array}$ & $\begin{array}{l}12.0 \\
13.5\end{array}$ & $\begin{array}{l}13.5 \\
14.0\end{array}$ & $\begin{array}{l}13.5 \\
14.0\end{array}$ & $\begin{array}{l}13.5 \\
14.0\end{array}$ & $\begin{array}{l}13.5 \\
40.0\end{array}$ & $\begin{array}{l}\text { helium } \\
304 \text { sst }\end{array}$ & $\begin{array}{l}\text { helium } \\
304 \text { sst }\end{array}$ & $\begin{array}{l}0.0 \\
0.0\end{array}$ & $\begin{array}{l}\text { none } \\
\text { sand-b }\end{array}$ \\
\hline A.6 & $\begin{array}{l}\text { stab } \\
\text { can }\end{array}$ & $\begin{array}{l}12.0 \\
13.5\end{array}$ & $\begin{array}{l}13.5 \\
15.5\end{array}$ & $\begin{array}{l}13.5 \\
15.5\end{array}$ & $\begin{array}{l}13.5 \\
15.5\end{array}$ & $\begin{array}{l}13.5 \\
40.0\end{array}$ & $\begin{array}{l}\text { helium } \\
304 \text { sst }\end{array}$ & $\begin{array}{l}\text { helium } \\
304 \text { st }\end{array}$ & $\begin{array}{l}0.0 \\
0.0\end{array}$ & $\begin{array}{l}\text { none } \\
\text { sand-b }\end{array}$ \\
\hline A.7 & $\begin{array}{l}\text { stab } \\
\text { can }\end{array}$ & $\begin{array}{l}12.0 \\
13.5\end{array}$ & $\begin{array}{l}13.5 \\
14.0\end{array}$ & $\begin{array}{l}13.5 \\
14.0\end{array}$ & $\begin{array}{l}13.5 \\
14.0\end{array}$ & $\begin{array}{l}13.5 \\
20.0\end{array}$ & $\begin{array}{l}\text { helium } \\
304 \text { sst }\end{array}$ & $\begin{array}{l}\text { helium } \\
\text { 304sst }\end{array}$ & $\begin{array}{l}0.0 \\
0.0\end{array}$ & $\begin{array}{l}\text { none } \\
\text { sand-b }\end{array}$ \\
\hline A.8 & $\begin{array}{l}\text { stab } \\
\text { can }\end{array}$ & $\begin{array}{l}12.0 \\
13.5\end{array}$ & $\begin{array}{l}13.5 \\
14.0\end{array}$ & $\begin{array}{l}13.5 \\
14.0\end{array}$ & $\begin{array}{l}13.5 \\
14.0\end{array}$ & $\begin{array}{l}13.5 \\
40.0\end{array}$ & $\begin{array}{l}\text { hei i um } \\
304 \text { sst }\end{array}$ & $\begin{array}{l}\text { nelium } \\
304 \text { sst }\end{array}$ & $\begin{array}{l}0.0 \\
0.0\end{array}$ & $\begin{array}{l}\text { none } \\
\text { clino }\end{array}$ \\
\hline A.9 & $\begin{array}{l}\text { stab } \\
\text { can }\end{array}$ & $\begin{array}{l}12.0 \\
13.5\end{array}$ & $\begin{array}{l}13.5 \\
14.0\end{array}$ & $\begin{array}{l}13.5 \\
14.0\end{array}$ & $\begin{array}{l}13.5 \\
14.0\end{array}$ & $\begin{array}{l}13.5 \\
20.0\end{array}$ & $\begin{array}{l}\text { zire } \\
\text { zire }\end{array}$ & $\begin{array}{l}\text { zire } \\
\text { zire }\end{array}$ & $\begin{array}{l}0.0 \\
0.0\end{array}$ & $\begin{array}{l}\text { none } \\
\text { sand-o }\end{array}$ \\
\hline A.10 & $\begin{array}{l}\text { stab } \\
\operatorname{can}\end{array}$ & $\begin{array}{l}12.0 \\
13.5\end{array}$ & $\begin{array}{l}13.5 \\
15.5\end{array}$ & $\begin{array}{l}13.5 \\
15.5\end{array}$ & $\begin{array}{l}13.5 \\
15.5\end{array}$ & $\begin{array}{l}13.5 \\
20.0\end{array}$ & $\begin{array}{l}\text { zire } \\
\text { zire }\end{array}$ & $\begin{array}{l}\text { zire } \\
\text { zire }\end{array}$ & $\begin{array}{l}0.0 \\
0.0\end{array}$ & $\begin{array}{l}\text { none } \\
\text { sand-b }\end{array}$ \\
\hline
\end{tabular}

Note: Unless otherwise noted, ail dimensions are in inches. 
Table 4-1. (Continued)

\begin{tabular}{|c|c|c|c|c|c|c|c|c|c|c|}
\hline \multirow[t]{2}{*}{ Concept } & \multirow[t]{2}{*}{ Element } & \multicolumn{2}{|c|}{$\begin{array}{l}\text { Inside } \\
\text { Material }\end{array}$} & \multicolumn{2}{|c|}{$\begin{array}{l}\text { Outside } \\
\text { Material }\end{array}$} & \multirow{2}{*}{$\begin{array}{r}\text { filler } \\
00\end{array}$} & \multirow[t]{2}{*}{$\mid \begin{array}{c}\text { Insida } \\
\text { Material }\end{array}$} & \multirow[t]{2}{*}{$\begin{array}{l}\text { Outside } \\
\text { Material }\end{array}$} & \multirow{2}{*}{$\begin{array}{c}\text { Coating } \\
\text { Delay } \\
\text { (yps) }\end{array}$} & \multirow[t]{2}{*}{ Plller } \\
\hline & & ID & 00 & 10 & 00 & & & & & \\
\hline 3.1 & $\begin{array}{l}\text { stab } \\
\text { can } \\
0 \text { pack }\end{array}$ & $\begin{array}{l}12.0 \\
13.5 \\
14.5\end{array}$ & $\begin{array}{l}13.5 \\
14.0 \\
15.0\end{array}$ & $\begin{array}{l}13.5 \\
14.0 \\
15.0\end{array}$ & $\begin{array}{l}13.5 \\
14.0 \\
15.0\end{array}$ & $\begin{array}{l}13.5 \\
14.5 \\
40.0\end{array}$ & $\begin{array}{l}\text { steel } \\
\text { steel } \\
304 \text { st }\end{array}$ & $\begin{array}{l}\text { steel } \\
\text { steel } \\
304 s 5 t\end{array}$ & $\begin{array}{l}0.0 \\
0.0 \\
0.0\end{array}$ & $\begin{array}{l}\text { none } \\
\text { sand-b } \\
\text { sand-b }\end{array}$ \\
\hline 3.2 & $\begin{array}{l}\text { stab } \\
\text { can } \\
\text { o pack }\end{array}$ & $\begin{array}{l}12.0 \\
13.5 \\
14.5\end{array}$ & $\begin{array}{l}13.5 \\
14.0 \\
16.5\end{array}$ & $\begin{array}{l}13.5 \\
14.0 \\
16.5\end{array}$ & $\begin{array}{l}13.5 \\
14.0 \\
16.5\end{array}$ & $\begin{array}{l}13.5 \\
14.5 \\
40.0\end{array}$ & $\begin{array}{l}\text { steel } \\
\text { steel } \\
304 s s t\end{array}$ & $\begin{array}{l}\text { steel } \\
\text { steel } \\
304 s s t\end{array}$ & $\begin{array}{l}0.0 \\
0.0 \\
0.0\end{array}$ & $\begin{array}{l}\text { none } \\
\text { sand-b } \\
\text { sand -b }\end{array}$ \\
\hline 8.3 & $\begin{array}{l}\text { stab } \\
\text { can } \\
\text { - pack }\end{array}$ & $\begin{array}{l}12.0 \\
13.5 \\
14.5\end{array}$ & $\begin{array}{l}13.5 \\
14.0 \\
15.0\end{array}$ & $\begin{array}{l}13.5 \\
14.0 \\
15.0\end{array}$ & $\begin{array}{l}13.5 \\
14.0 \\
15.0\end{array}$ & $\begin{array}{l}13.5 \\
14.5 \\
20.0\end{array}$ & $\begin{array}{l}\text { steel } \\
\text { steel } \\
304 s s t\end{array}$ & $\begin{array}{l}\text { steel } \\
\text { steel } \\
304 \text { st }\end{array}$ & $\begin{array}{l}0.0 \\
0.0 \\
0.0\end{array}$ & $\begin{array}{l}\text { none } \\
\text { sand-b } \\
\text { sand-b }\end{array}$ \\
\hline 8.4 & $\begin{array}{l}\text { stab } \\
\text { can } \\
0 \text { pack }\end{array}$ & $\begin{array}{l}12.0 \\
13.5 \\
14.5\end{array}$ & $\begin{array}{l}13.5 \\
14.0 \\
16.5\end{array}$ & $\begin{array}{l}13.5 \\
14.0 \\
16.5\end{array}$ & $\begin{array}{l}13.5 \\
14.0 \\
16.5\end{array}$ & $\begin{array}{l}13.5 \\
14.5 \\
20.0\end{array}$ & $\begin{array}{l}\text { steel } \\
\text { steel } \\
30455 t\end{array}$ & $\begin{array}{l}\text { strel } \\
\text { steel } \\
3045 s t\end{array}$ & $\begin{array}{l}0.0 \\
0.0 \\
0.0\end{array}$ & $\begin{array}{l}\text { none } \\
\text { sand-b } \\
\text { sand-b }\end{array}$ \\
\hline 3.5 & $\begin{array}{l}\text { stab } \\
\text { can } \\
0 \text { pack }\end{array}$ & $\begin{array}{l}12.0 \\
13.5 \\
14.5\end{array}$ & $\begin{array}{l}13.5 \\
14.0 \\
15.0\end{array}$ & $\begin{array}{l}13.5 \\
14.0 \\
15.0\end{array}$ & $\begin{array}{l}13.5 \\
14.0 \\
15.0\end{array}$ & $\begin{array}{l}13.5 \\
14.5 \\
20.0\end{array}$ & $\begin{array}{l}\text { steel } \\
\text { steel } \\
304 \text { st }\end{array}$ & $\begin{array}{l}\text { steel } \\
\text { steel } \\
304 s s t\end{array}$ & $\begin{array}{l}0.0 \\
0.0 \\
0.0\end{array}$ & $\begin{array}{l}\text { none } \\
\text { sand-b } \\
\text { clino }\end{array}$ \\
\hline 8.6 & $\begin{array}{l}\text { stab } \\
\text { can } \\
0 \text { pack }\end{array}$ & $\begin{array}{l}12.0 \\
13.5 \\
14.5\end{array}$ & $\begin{array}{l}13.5 \\
14.0 \\
16.5\end{array}$ & $\begin{array}{l}13.5 \\
14.0 \\
16.5\end{array}$ & $\begin{array}{l}13.5 \\
14.0 \\
16.5\end{array}$ & $\begin{array}{l}13.5 \\
14.5 \\
20.0\end{array}$ & $\begin{array}{l}\text { steel } \\
\text { steel } \\
304 \text { st }\end{array}$ & $\begin{array}{l}\text { steel } \\
\text { steel } \\
30455 t\end{array}$ & $\begin{array}{l}0.0 \\
0.0 \\
0.0\end{array}$ & $\begin{array}{l}\text { none } \\
\text { sand-b } \\
\text { clino }\end{array}$ \\
\hline 3.7 & $\begin{array}{l}\text { stab } \\
\text { can } \\
0 \text { pack }\end{array}$ & $\begin{array}{l}12.0 \\
13.5 \\
14.5\end{array}$ & $\begin{array}{l}13.5 \\
14.0 \\
15.0\end{array}$ & $\begin{array}{l}13.5 \\
14.0 \\
15.0\end{array}$ & $\begin{array}{l}13.5 \\
14.0 \\
15.0\end{array}$ & $\begin{array}{l}13.5 \\
14.5 \\
20.0\end{array}$ & $\begin{array}{l}\text { steel } \\
\text { steel } \\
\text { zire }\end{array}$ & $\begin{array}{l}\text { steel } \\
\text { steel } \\
\text { zirc }\end{array}$ & $\begin{array}{l}0.0 \\
0.0 \\
0.0\end{array}$ & $\begin{array}{l}\text { none } \\
\text { sand-b } \\
\text { sand-b }\end{array}$ \\
\hline 3.8 & $\left\{\begin{array}{l}\text { tab } \\
\text { can } \\
0 \text { pack }\end{array}\right.$ & $\begin{array}{l}12.0 \\
13.5 \\
14.5\end{array}$ & $\begin{array}{l}13.5 \\
14.0 \\
16.5\end{array}$ & $\begin{array}{l}13.5 \\
14.0 \\
16.5\end{array}$ & $\begin{array}{l}13.5 \\
14.0 \\
16.5\end{array}$ & $\begin{array}{l}13.5 \\
14.5 \\
20.0\end{array}$ & $\begin{array}{l}\text { steel } \\
\text { steel } \\
\text { zipe }\end{array}$ & $\begin{array}{l}\text { steel } \\
\text { steel } \\
\text { zirc }\end{array}$ & $\begin{array}{l}0.0 \\
0.0 \\
0.0\end{array}$ & $\begin{array}{l}\text { none } \\
\text { sand-b } \\
\text { sand-b }\end{array}$ \\
\hline 3.9 & $\begin{array}{l}\text { stab } \\
\text { can } \\
0 \text { pack }\end{array}$ & $\begin{array}{l}12.0 \\
13.5 \\
14.5\end{array}$ & $\begin{array}{l}13.5 \\
14.0 \\
15.0\end{array}$ & $\begin{array}{l}13.5 \\
14.0 \\
15.0\end{array}$ & $\begin{array}{l}13.5 \\
14.0 \\
15.0\end{array}$ & $\begin{array}{l}13.5 \\
14.5 \\
20.0\end{array}$ & $\begin{array}{l}\text { steel } \\
\text { steel } \\
\text { copper }\end{array}$ & $\begin{array}{l}\text { steel } \\
\text { steel } \\
\text { copper }\end{array}$ & $\begin{array}{l}0.0 \\
0.0 \\
0.0\end{array}$ & $\begin{array}{l}\text { none } \\
\text { sand-b } \\
\text { sand-b }\end{array}$ \\
\hline
\end{tabular}


Table 4-1. (Continued)

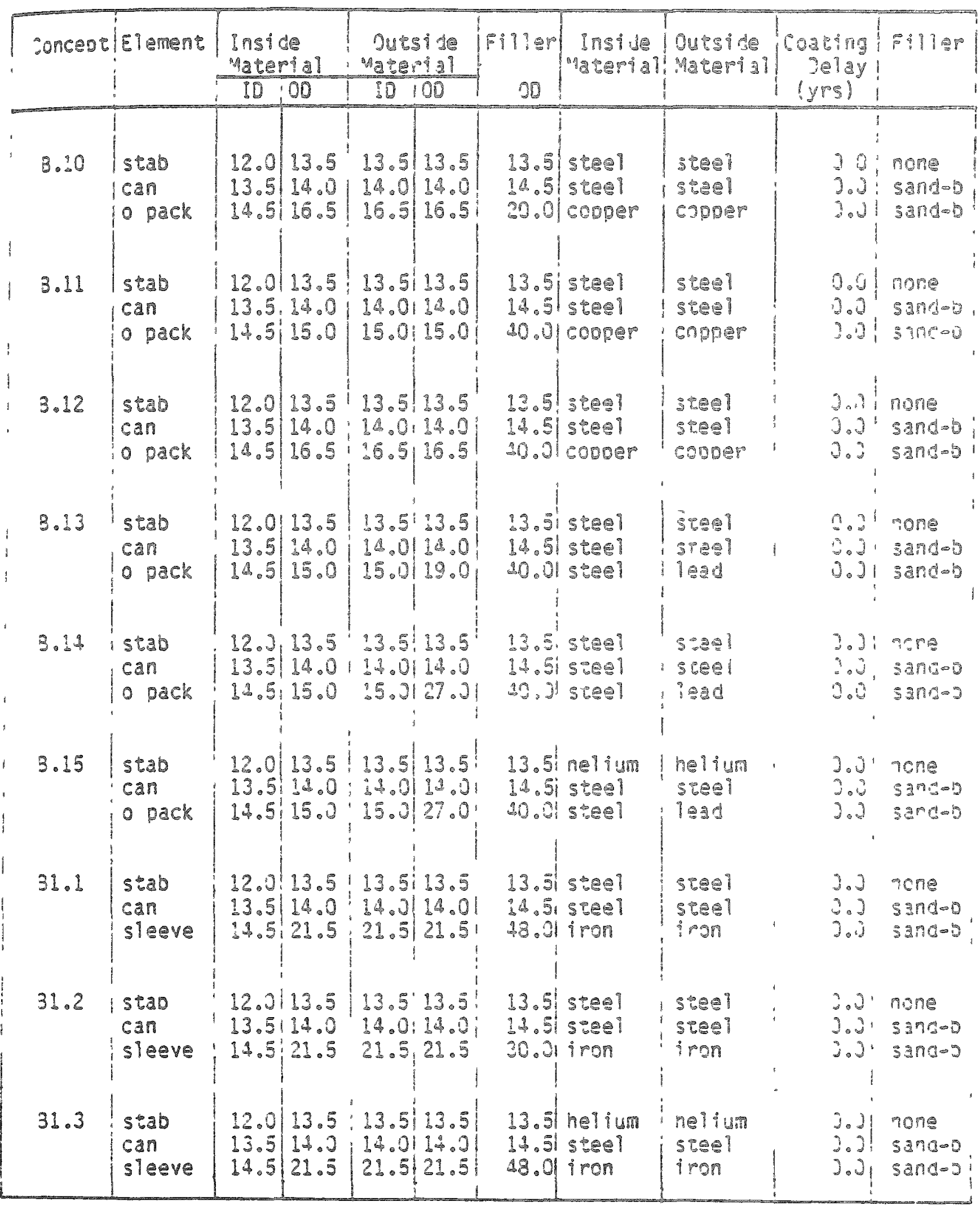


Tabt: 4-1. (Gm inued)

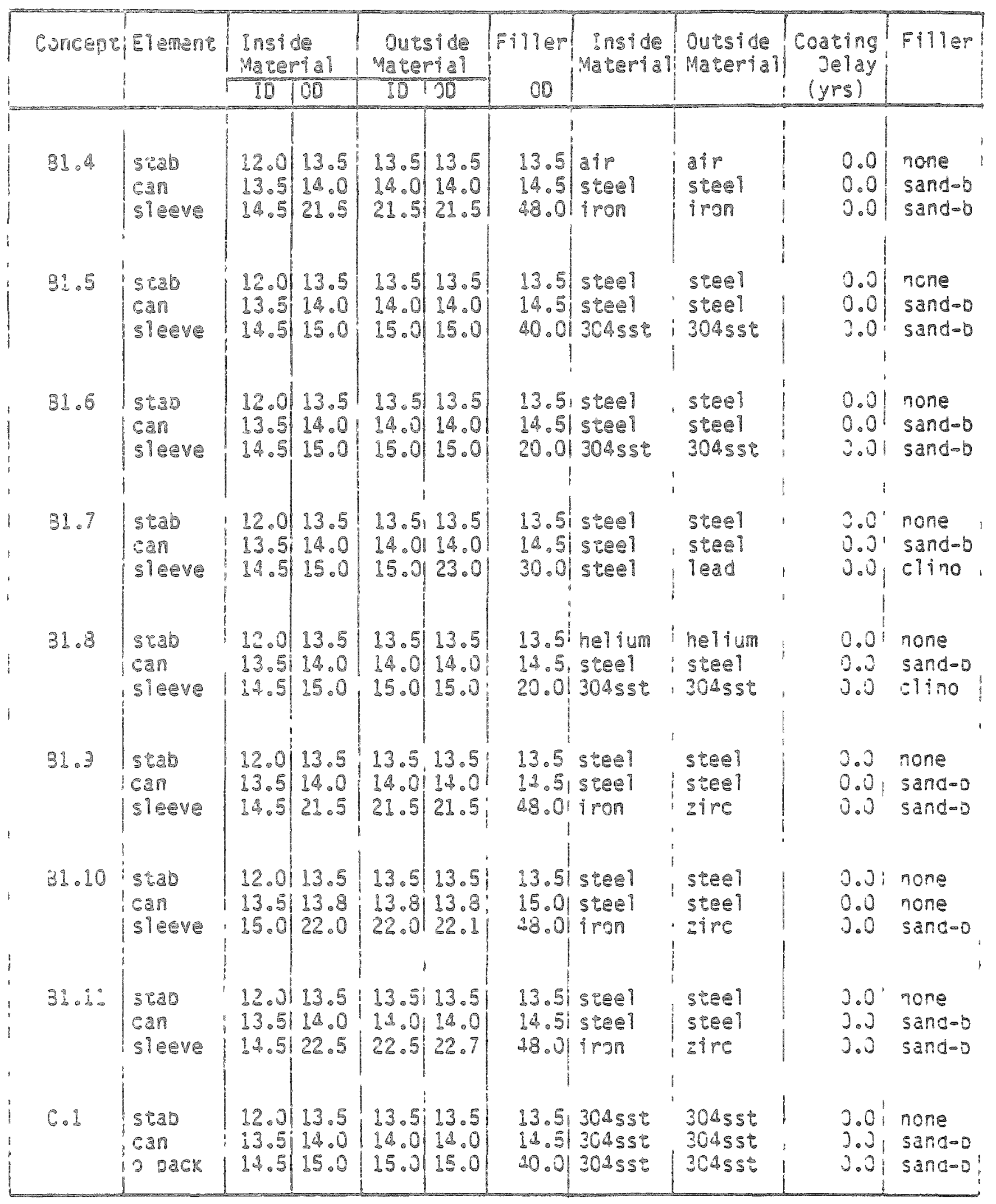


Table 4-1. (Continued)

\begin{tabular}{|c|c|c|c|c|c|c|c|c|c|c|}
\hline \multirow[t]{2}{*}{ Concept } & \multirow[t]{2}{*}{ Element } & \multicolumn{2}{|c|}{$\begin{array}{l}\text { Inside } \\
\text { Material }\end{array}$} & \multicolumn{2}{|c|}{$\begin{array}{c}\text { Outside } \\
\text { Material }\end{array}$} & \multirow{2}{*}{$\begin{array}{c}\text { Filler } \\
00\end{array}$} & \multirow[t]{2}{*}{$\begin{array}{l}\text { Inside } \\
\text { Material }\end{array}$} & \multirow[t]{2}{*}{$\begin{array}{l}\text { Outside } \\
\text { Material }\end{array}$} & \multirow{2}{*}{$\begin{array}{c}\text { Coating } \\
\text { Delay } \\
\text { (yps) }\end{array}$} & \multirow[t]{2}{*}{ Filler } \\
\hline & & ID & 00 & ID & 00 & & & & & \\
\hline C.2 & $\begin{array}{l}\text { stab } \\
\text { can } \\
\text { o pack }\end{array}$ & $\begin{array}{l}12.0 \\
13.5 \\
16.0\end{array}$ & $\begin{array}{l}13.5 \\
15.5 \\
18.0\end{array}$ & $\begin{array}{l}13.5 \\
15.5 \\
18.0\end{array}$ & $\begin{array}{l}13.5 \\
15.5 \\
18.0\end{array}$ & $\begin{array}{l}13.5 \\
16.0 \\
40.0\end{array}$ & $\begin{array}{l}304 s s t \\
304 s s t \\
304 s s t\end{array}$ & $\begin{array}{l}304 s s t \\
3045 s t \\
304 s s t\end{array}$ & $\begin{array}{l}0.0 \\
0.0 \\
0.0\end{array}$ & $\begin{array}{l}\text { none } \\
\text { sand-b } \\
\text { sand-b }\end{array}$ \\
\hline C.3 & $\begin{array}{l}\text { stab } \\
\text { can } \\
\text { o pack }\end{array}$ & $\begin{array}{l}12.0 \\
13.5 \\
16.0\end{array}$ & $\begin{array}{l}13.5 \\
15.5 \\
18.0\end{array}$ & $\begin{array}{l}13.5 \\
15.5 \\
18.0\end{array}$ & $\begin{array}{l}13.5 \\
15.5 \\
18.0\end{array}$ & $\begin{array}{l}13.5 \\
16.0 \\
20.0\end{array}$ & $\begin{array}{l}304 s s t \\
304 s s t \\
304 s s t\end{array}$ & $\begin{array}{l}3045 s t \\
30455 t \\
3045 s t\end{array}$ & $\begin{array}{l}0.0 \\
0.0 \\
0.0\end{array}$ & $\begin{array}{l}\text { none } \\
\text { sand-b } \\
\text { sand-b }\end{array}$ \\
\hline C.4 & $\begin{array}{l}\text { stab } \\
\text { can } \\
\text { o pack }\end{array}$ & $\begin{array}{l}12.0 \\
13.5 \\
14.5\end{array}$ & $\begin{array}{l}13.5 \\
14.0 \\
15.0\end{array}$ & $\begin{array}{l}13.5 \\
14.0 \\
15.0\end{array}$ & $\begin{array}{l}13.5 \\
14.0 \\
15.0\end{array}$ & $\begin{array}{l}13.5 \\
14.5 \\
20.0\end{array}$ & $\begin{array}{l}\text { zire } \\
\text { zire } \\
\text { zire }\end{array}$ & $\begin{array}{l}\text { zire } \\
\text { zire } \\
\text { zire }\end{array}$ & $\begin{array}{l}0.0 \\
0.0 \\
0.0\end{array}$ & $\begin{array}{l}\text { none } \\
\text { sand-b } \\
\text { sand-b }\end{array}$ \\
\hline C.5 & $\begin{array}{l}\text { stab } \\
\text { can } \\
0 \text { pack }\end{array}$ & $\begin{array}{l}12.0 \\
13.5 \\
16.0\end{array}$ & $\begin{array}{l}13.5 \\
15.5 \\
18.0\end{array}$ & $\begin{array}{l}13.5 \\
15.5 \\
18.0\end{array}$ & $\begin{array}{l}13.5 \\
15.5 \\
18.0\end{array}$ & $\begin{array}{l}13.5 \\
16.0 \\
20.0\end{array}$ & $\begin{array}{l}\text { zire } \\
\text { zire } \\
\text { zire }\end{array}$ & $\begin{array}{l}\text { zire } \\
\text { zire } \\
\text { zire }\end{array}$ & $\begin{array}{l}0.0 \\
0.0 \\
0.0\end{array}$ & $\begin{array}{l}\text { none } \\
\text { sand }=0 \\
\text { sand-b }\end{array}$ \\
\hline 0.6 & $\begin{array}{l}\text { stab } \\
\text { can } \\
0 \text { pack }\end{array}$ & $\begin{array}{l}12.0 \\
13.5 \\
16.0\end{array}$ & $\begin{array}{l}13.5 \\
15.5 \\
16.5\end{array}$ & $\begin{array}{l}13.5 \\
15.5 \\
16.5\end{array}$ & $\begin{array}{l}13.5 \\
15.5 \\
24.5\end{array}$ & $\begin{array}{l}13.5 \\
16.0 \\
30.0\end{array}$ & $\begin{array}{l}304 s s t \\
304 s s t \\
304 s s t\end{array}$ & $\begin{array}{l}3045 s t \\
30455 t \\
\text { lead }\end{array}$ & $\begin{array}{l}0.0 \\
0.0 \\
0.0\end{array}$ & $\begin{array}{l}\text { none } \\
\text { sand-b } \\
\text { sand-b }\end{array}$ \\
\hline 0.7 & $\begin{array}{l}\text { stab } \\
\text { can } \\
0 \text { pack }\end{array}$ & $\begin{array}{l}12.0 \\
13.5 \\
16.0\end{array}$ & $\begin{array}{l}13.5 \\
15.5 \\
18.0\end{array}$ & $\begin{array}{l}13.5 \\
15.5 \\
18.0\end{array}$ & $\begin{array}{l}13.5 \\
15.5 \\
18.0\end{array}$ & $\begin{array}{l}13.5 \\
16.0 \\
40.0\end{array}$ & $\begin{array}{l}\text { helium } \\
\text { zirc } \\
\text { zirc }\end{array}$ & $\begin{array}{l}\text { helium } \\
\text { zire } \\
\text { zirc }\end{array}$ & $\begin{array}{l}0.0 \\
0.0 \\
0.0\end{array}$ & $\begin{array}{l}\text { none } \\
\text { sand-D } \\
\text { sand-b }\end{array}$ \\
\hline Cl.1 & $\begin{array}{l}\text { stab } \\
\text { can } \\
\text { o pack } \\
\text { sleeve }\end{array}$ & $\begin{array}{l}12.0 \\
13.5 \\
14.5 \\
15.5\end{array}$ & $\begin{array}{l}13.5 \\
14.0 \\
15.0 \\
22.5\end{array}$ & $\begin{array}{l}13.5 \\
14.0 \\
15.0 \\
22.5\end{array}$ & $\begin{array}{l}13.5 \\
14.0 \\
15.0 \\
22.5\end{array}$ & $\begin{array}{l}13.5 \\
14.5 \\
15.5 \\
45.0\end{array}$ & $\begin{array}{l}\text { inconel } \\
\text { inconel } \\
3045 s t \\
\text { iron }\end{array}$ & $\begin{array}{l}\text { inconel } \\
\text { inconel } \\
30455 t \\
\text { iron }\end{array}$ & $\begin{array}{l}0.0 \\
0.0 \\
0.0 \\
0.0\end{array}$ & $\begin{array}{l}\text { none } \\
\text { sand-b } \\
\text { sand-b } \\
\text { sand-b }\end{array}$ \\
\hline C1.2 & $\begin{array}{l}\text { stab } \\
\text { can } \\
\text { o pack } \\
\text { sleeve }\end{array}$ & $\begin{array}{l}12.0 \\
13.5 \\
14.5 \\
15.5\end{array}$ & $\begin{array}{l}13.5 \\
14.0 \\
15.0 \\
22.5\end{array}$ & $\begin{array}{l}13.5 \\
14.0 \\
15.0 \\
22.5\end{array}$ & $\begin{array}{l}13.5 \\
14.0 \\
15.0 \\
22.5\end{array}$ & $\begin{array}{l}13.5 \\
14.5 \\
15.5 \\
30.0\end{array}$ & $\begin{array}{l}\text { inconel } \\
\text { inconel } \\
3045 s t \\
\text { iron }\end{array}$ & $\begin{array}{l}\text { inconel } \\
\text { inconel } \\
3045 s t \\
\text { iron }\end{array}$ & $\begin{array}{l}0.0 \\
0.0 \\
0.0 \\
0.0\end{array}$ & $\begin{array}{l}\text { none } \\
\text { sand-b } \\
\text { sand-b } \\
\text { sand-b }\end{array}$ \\
\hline 61.3 & stá & 12.0 & 13.5 & 13.5 & 13.5 & 13.5 & helium & helium & 0.0 & none \\
\hline
\end{tabular}


Table 4-1. (Continued)

\begin{tabular}{|c|c|c|c|c|c|c|c|c|c|c|}
\hline \multirow[t]{2}{*}{ Concept } & \multirow[t]{2}{*}{ Element } & \multicolumn{2}{|c|}{$\begin{array}{l}\text { Inside } \\
\text { Material }\end{array}$} & \multicolumn{2}{|c|}{$\begin{array}{c}\text { Outside } \\
\text { Material }\end{array}$} & \multirow{2}{*}{$\begin{array}{r}\text { Finler } \\
00\end{array}$} & \multirow[t]{2}{*}{$\begin{array}{l}\text { Inside } \\
\text { Material }\end{array}$} & \multirow[t]{2}{*}{$\begin{array}{l}\text { Outside } \\
\text { Materiai }\end{array}$} & \multirow{2}{*}{$\begin{array}{c}\text { Coating } \\
\text { Delay } \\
\text { (yrs) }\end{array}$} & \multirow[t]{2}{*}{ Filler } \\
\hline & & 1010 & 00 & ID & 00 & & & & & \\
\hline C1.3 & $\begin{array}{l}\text { can } \\
\text { o pack } \\
\text { sleeve }\end{array}$ & $\begin{array}{l}13.5 \\
14.5 \\
15.5\end{array}$ & $\begin{array}{l}14.0 \\
15.0 \\
22.5\end{array}$ & $\begin{array}{l}14.0 \\
15.0 \\
22.5\end{array}$ & $\begin{array}{l}14.0 \\
15.0 \\
22.5\end{array}$ & $\begin{array}{l}14.5 \\
15.5 \\
45.0\end{array}$ & $\begin{array}{l}\text { inconel } \\
3045 s t \\
\text { iron }\end{array}$ & $\begin{array}{l}\text { inconel } \\
3045 s t \\
\text { iron }\end{array}$ & $\begin{array}{l}0.0 \\
0.0 \\
0.0\end{array}$ & $\begin{array}{l}\text { sand-b } \\
\text { sand }-b \\
\operatorname{sand}-b\end{array}$ \\
\hline Cl.4 & $\begin{array}{l}\text { stab } \\
\text { can } \\
\text { opack } \\
\text { sleeve }\end{array}$ & $\begin{array}{l}12.0 \\
13.5 \\
14.5 \\
15.5\end{array}$ & $\begin{array}{l}13.5 \\
14.0 \\
15.0 \\
16.5\end{array}$ & $\begin{array}{l}13.5 \\
14.0 \\
15.0 \\
16.5\end{array}$ & $\begin{array}{l}13.5 \\
14.0 \\
15.0 \\
16.5\end{array}$ & $\begin{array}{l}13.5 \\
14.5 \\
15.5 \\
40.0\end{array}$ & $\begin{array}{l}304 \text { sst } \\
304 s s t \\
304 s s t \\
\text { steel }\end{array}$ & $\begin{array}{l}304 \text { sst } \\
304 \text { st } \\
304 \text { sst } \\
\text { steel }\end{array}$ & $\begin{array}{l}0.0 \\
0.0 \\
0.0 \\
0.0\end{array}$ & $\begin{array}{l}\text { none } \\
\text { sand-b } \\
\text { sand-b } \\
\text { sand-b }\end{array}$ \\
\hline$C 1.5$ & $\begin{array}{l}\text { stab } \\
\text { can } \\
\text { o pack } \\
\text { sleeve }\end{array}$ & $\begin{array}{l}12.0 \\
13.5 \\
14.5 \\
15.5\end{array}$ & $\begin{array}{l}13.5 \\
14.0 \\
15.0 \\
16.5\end{array}$ & $\begin{array}{l}13.5 \\
14.0 \\
15.0 \\
16.5\end{array}$ & $\begin{array}{l}13.5 \\
14.0 \\
15.0 \\
16.5\end{array}$ & $\begin{array}{l}13.5 \\
14.5 \\
15.5 \\
24.0\end{array}$ & $\begin{array}{l}3045 s t \\
3045 s t \\
3045 s t \\
\text { steel }\end{array}$ & $\begin{array}{l}304 \text { sst } \\
304 s s t \\
304 s s t \\
\text { steel }\end{array}$ & $\begin{array}{l}0.0 \\
0.0 \\
0.0 \\
0.0\end{array}$ & $\begin{array}{l}\text { none } \\
\text { sand-b } \\
\text { sand-b } \\
\text { sand-b }\end{array}$ \\
\hline C1.6 & $\begin{array}{l}\text { stab } \\
\text { can } \\
\text { o pack } \\
\text { sleeve }\end{array}$ & \begin{tabular}{l|}
12.0 \\
13.5 \\
14.5 \\
15.5
\end{tabular} & $\begin{array}{l}13.5 \\
14.0 \\
15.0 \\
16.5\end{array}$ & $\begin{array}{l}13.5 \\
14.0 \\
15.0 \\
16.5\end{array}$ & $\begin{array}{l}13.5 \\
14.0 \\
15.0 \\
16.5\end{array}$ & $\begin{array}{l}13.5 \\
14.5 \\
15.5 \\
24.0\end{array}$ & $\begin{array}{l}\text { zirc } \\
\text { zirc } \\
\text { zire } \\
\text { steel }\end{array}$ & $\begin{array}{l}\text { zire } \\
\text { zire } \\
\text { zire } \\
\text { steel }\end{array}$ & $\begin{array}{l}0.0 \\
0.0 \\
0.0 \\
0.0\end{array}$ & $\begin{array}{l}\text { none } \\
\text { sand-b } \\
\text { sand-b } \\
\text { sand-b }\end{array}$ \\
\hline 0.1 & $\begin{array}{l}\text { stab } \\
\text { can } \\
\text { sleeve }\end{array}$ & $\begin{array}{l}12.0 \\
13.5 \\
14.5\end{array}$ & $\begin{array}{l}13.5 \\
14.0 \\
21.5\end{array}$ & $\begin{array}{l}13.5 \\
14.0 \\
21.5\end{array}$ & $\begin{array}{l}13.5 \\
14.0 \\
21.5\end{array}$ & $\begin{array}{l}13.5 \\
14.5 \\
48.0\end{array}$ & $\begin{array}{l}\text { helium } \\
\text { steel } \\
\text { iron }\end{array}$ & $\begin{array}{l}\text { helium } \\
\text { steel } \\
\text { iron }\end{array}$ & $\begin{array}{r}0.0 \\
0.0 \\
10.0\end{array}$ & $\begin{array}{l}\text { none } \\
\text { sand }-b \\
\text { sand }-b\end{array}$ \\
\hline 0.2 & $\begin{array}{l}\text { stab } \\
\text { can } \\
\text { sleeve }\end{array}$ & $\begin{array}{l}12.0 \\
13.5 \\
14.5\end{array}$ & $\begin{array}{l}13.5 \\
14.0 \\
21.5\end{array}$ & $\begin{array}{l}13.5 \\
14.0 \\
21.5\end{array}$ & $\begin{array}{l}13.5 \\
14.0 \\
21.5\end{array}$ & $\begin{array}{l}13.5 \\
14.5 \\
48.0\end{array}$ & $\begin{array}{l}\text { steel } \\
\text { steel } \\
\text { iron }\end{array}$ & $\begin{array}{l}\text { steel } \\
\text { steel } \\
\text { iron }\end{array}$ & $\begin{array}{r}0.0 \\
0.0 \\
10.0\end{array}$ & $\begin{array}{l}\text { none } \\
\text { sand-b } \\
\text { sand-b }\end{array}$ \\
\hline 0.3 & $\begin{array}{l}\text { stab } \\
\text { can } \\
\text { sleeve }\end{array}$ & $\begin{array}{l}12.0 \\
13.5 \\
14.5\end{array}$ & $\begin{array}{l}13.5 \\
14.0 \\
23.5\end{array}$ & $\begin{array}{l}13.5 \\
14.9 \\
21.5\end{array}$ & $\begin{array}{l}13.5 \\
14.0 \\
21.5\end{array}$ & $\begin{array}{l}13.5 \\
14.5 \\
48.0\end{array}$ & $\begin{array}{l}\text { steel } \\
\text { steel } \\
\text { iron }\end{array}$ & $\begin{array}{l}\text { steel } \\
\text { steel } \\
\text { iron }\end{array}$ & $\begin{array}{r}0.0 \\
0.0 \\
100.0\end{array}$ & $\begin{array}{l}\text { none } \\
\text { sand-b } \\
\text { sand-b }\end{array}$ \\
\hline 0.4 & $\begin{array}{l}\text { stab } \\
\text { can } \\
\text { sleeve }\end{array}$ & $\begin{array}{l}12.0 \\
13.5 \\
14.5\end{array}$ & $\begin{array}{l}13.5 \\
14.0 \\
16.5\end{array}$ & $\begin{array}{l}13.5 \\
14.0 \\
16.5\end{array}$ & $\begin{array}{l}13.5 \\
14.0 \\
16.5\end{array}$ & $\begin{array}{l}13.5 \\
14.5 \\
24.0\end{array}$ & $\begin{array}{l}\text { steel } \\
\text { steel } \\
\text { zirc }\end{array}$ & $\begin{array}{l}\text { steel } \\
\text { steel } \\
\text { zirs }\end{array}$ & $\begin{array}{r}0.0 \\
0.0 \\
100.0\end{array}$ & $\begin{array}{l}\text { none } \\
\text { sand-b } \\
\text { sand-b }\end{array}$ \\
\hline
\end{tabular}


Table 4-1. (Continued)

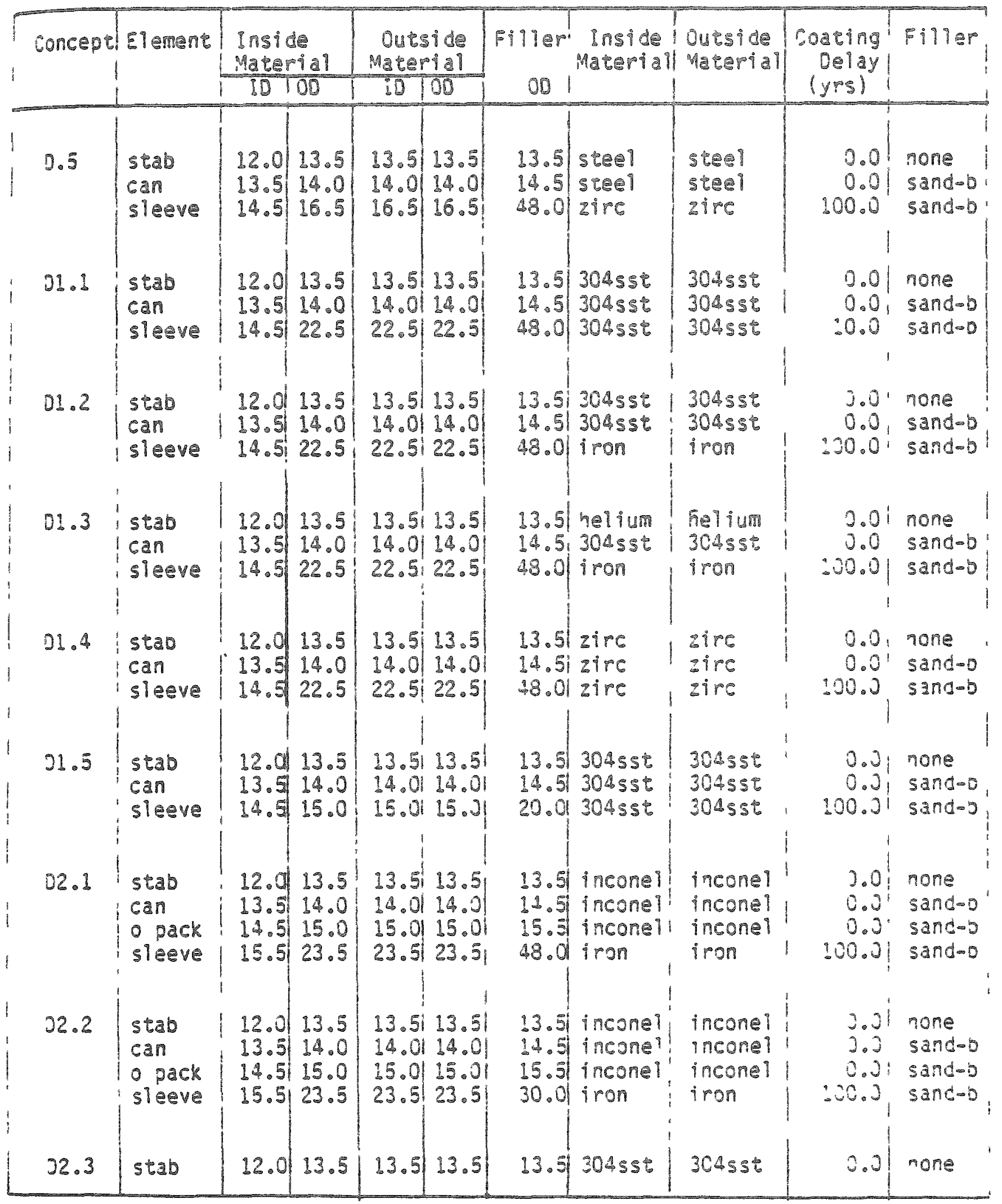


Table 4-1. (Continued)

\begin{tabular}{|c|c|c|c|c|c|c|c|c|c|c|}
\hline \multirow[t]{2}{*}{ Concept } & \multirow[t]{2}{*}{ Element } & \multicolumn{2}{|c|}{$\begin{array}{l}\text { Inside } \\
\text { Material }\end{array}$} & \multicolumn{2}{|c|}{$\begin{array}{l}\text { Outside } \\
\text { Material }\end{array}$} & \multirow{2}{*}{$\begin{array}{c}\text { Filier } \\
00\end{array}$} & \multirow[t]{2}{*}{$\begin{array}{c}\text { Inside } \\
\text { Material }\end{array}$} & \multirow[t]{2}{*}{$\begin{array}{l}\text { Outside } \\
\text { Material }\end{array}$} & \multirow{2}{*}{$\begin{array}{c}\text { Coating } \\
\text { Delay } \\
(\text { yrs) }\end{array}$} & \multirow[t]{2}{*}{ Filler } \\
\hline & & TD & 00 & 10 & 00 & & & & & \\
\hline 02.3 & $\begin{array}{l}\text { can } \\
\text { o pack } \\
\text { sleeve }\end{array}$ & $\begin{array}{l}13.5 \\
14.5 \\
15.5\end{array}$ & $\begin{array}{l}14.0 \\
15.0 \\
23.5\end{array}$ & $\begin{array}{l}14.0 \\
15.0 \\
23.5\end{array}$ & $\begin{array}{l}14.0 \\
15.0 \\
23.5\end{array}$ & $\begin{array}{l}14.5 \\
15.5 \\
30.0\end{array}$ & $\begin{array}{l}304 \text { sst } \\
304 \text { sst } \\
\text { iron }\end{array}$ & $\begin{array}{l}3045 s t \\
3045 s t \\
\text { iron }\end{array}$ & $\begin{array}{r}0.0 \\
0.0 \\
100.0\end{array}$ & $\begin{array}{l}\text { sand }-b \\
\text { sand-b } \\
\text { sand-b }\end{array}$ \\
\hline 02.4 & $\begin{array}{l}\text { stab } \\
\text { can } \\
\text { o pack } \\
\text { sleeve }\end{array}$ & $\begin{array}{l}12.0 \\
13.5 \\
14.5 \\
15.5\end{array}$ & $\begin{array}{l}13.5 \\
14.0 \\
15.0 \\
16.0\end{array}$ & $\begin{array}{l}13.5 \\
14.0 \\
15.0 \\
16.0\end{array}$ & $\begin{array}{l}13.5 \\
14.0 \\
15.0 \\
16.0\end{array}$ & $\begin{array}{l}13.5 \\
14.5 \\
15.5 \\
48.0\end{array}$ & $\begin{array}{l}\text { hel ium } \\
30455 t \\
3045 s t \\
304 s 5 t\end{array}$ & $\begin{array}{l}\text { helium } \\
3045 s t \\
3045 s t \\
304 s s t\end{array}$ & $\begin{array}{r}0.0 \\
0.0 \\
0.0 \\
100.0\end{array}$ & $\begin{array}{l}\text { none } \\
\text { sand-b } \\
\text { sand-b } \\
\text { clino }\end{array}$ \\
\hline 02.5 & $\begin{array}{l}\text { stab } \\
\text { can } \\
\text { o pack } \\
\text { sleeve }\end{array}$ & $\begin{array}{l}12.0 \\
13.5 \\
14.5 \\
23.5\end{array}$ & $\begin{array}{l}13.5 \\
14.0 \\
15.0 \\
24.0\end{array}$ & $\begin{array}{l}13.5 \\
14.0 \\
15.0 \\
24.0\end{array}$ & $\begin{array}{l}13.5 \\
14.0 \\
23.0 \\
24.0\end{array}$ & $\begin{array}{l}13.5 \\
14.5 \\
23.5 \\
48.0\end{array}$ & $\begin{array}{l}\text { helium } \\
304 \text { sst } \\
304 s 5 t \\
304 s s t\end{array}$ & $\begin{array}{l}\text { helium } \\
304 \text { sst } \\
\text { lead } \\
304 \text { sst }\end{array}$ & $\begin{array}{r}0.0 \\
0.0 \\
0.0 \\
100.0\end{array}$ & $\begin{array}{l}\text { none } \\
\text { sand-b } \\
\text { sand-b } \\
\text { clino }\end{array}$ \\
\hline 02.6 & $\begin{array}{l}\text { stab } \\
\text { can } \\
\text { o pack } \\
\text { sleeve }\end{array}$ & $\begin{array}{l}12.0 \\
13.5 \\
14.5 \\
27.5\end{array}$ & $\begin{array}{l}13.5 \\
14.0 \\
15.0 \\
28.0\end{array}$ & $\begin{array}{l}13.5 \\
14.0 \\
15.0 \\
28.0\end{array}$ & $\begin{array}{l}13.5 \\
14.0 \\
27.0 \\
28.0\end{array}$ & $\begin{array}{l}13.5 \\
14.5 \\
27.5 \\
48.0\end{array}$ & $\begin{array}{l}\text { helium } \\
\text { 304sst } \\
304 s s t \\
304 s s t\end{array}$ & $\begin{array}{l}\text { helium } \\
\text { 304sst } \\
\text { lead } \\
\text { 304sst }\end{array}$ & $\begin{array}{r}0.0 \\
0.0 \\
0.0 \\
100.0\end{array}$ & $\begin{array}{l}\text { none } \\
\text { sand-b } \\
\text { sand-b } \\
\text { clino }\end{array}$ \\
\hline 02.7 & $\begin{array}{l}\text { stab } \\
\text { can } \\
\text { o pack } \\
\text { sieeve }\end{array}$ & $\begin{array}{l}12.0 \\
13.5 \\
14.5 \\
27.5\end{array}$ & $\begin{array}{l}13.5 \\
14.0 \\
15.0 \\
28.0\end{array}$ & $\begin{array}{l}13.5 \\
14.0 \\
15.0 \\
28.0\end{array}$ & $\begin{array}{l}13.5 \\
14.0 \\
27.0 \\
28.0\end{array}$ & $\begin{array}{l}13.5 \\
14.5 \\
27.5 \\
48.0\end{array}$ & $\begin{array}{l}304 s s t \\
304 s s t \\
304 s s t \\
304 s s t\end{array}$ & $\begin{array}{l}304 s s t \\
304 s s t \\
1 \text { ead } \\
304 s s t\end{array}$ & $\begin{array}{r}0.0 \\
0.0 \\
0.0 \\
100.0\end{array}$ & $\begin{array}{l}\text { none } \\
\text { sand-b } \\
\text { sand-b } \\
\text { clino }\end{array}$ \\
\hline 02.8 & $\begin{array}{l}\text { stab } \\
\text { can } \\
\text { o pack } \\
\text { sleeve }\end{array}$ & $\begin{array}{l}12.0 \\
13.5 \\
14.5 \\
27.5\end{array}$ & $\begin{array}{l}13.5 \\
14.0 \\
15.0 \\
28.0\end{array}$ & $\begin{array}{l}13.5 \\
14.0 \\
15.0 \\
28.0\end{array} \mid$ & $\begin{array}{l}13.5 \\
14.0 \\
27.0 \\
28.0\end{array}$ & $\begin{array}{l}13.5 \\
14.5 \\
27.5 \\
48.0\end{array}$ & $\begin{array}{l}\text { zire } \\
\text { zire } \\
\text { zire } \\
\text { zire }\end{array}$ & $\begin{array}{l}\text { zire } \\
\text { zire } \\
\text { lead } \\
\text { zipe }\end{array}$ & $\begin{array}{r}0.0 \\
0.0 \\
0.0 \\
100.0\end{array}$ & $\begin{array}{l}\text { none } \\
\text { sand-b } \\
\text { sand-b } \\
\text { slino }\end{array}$ \\
\hline E.I & $\begin{array}{l}\text { stab } \\
\text { stab } \\
\text { can }\end{array}$ & $\begin{array}{l}12.0 \\
12.0 \\
13.5\end{array}$ & $\begin{array}{l}12.0 \\
12.0 \\
14.0\end{array}$ & $\begin{array}{l}12.0 \\
12.0 \\
14.0\end{array}$ & $\begin{array}{l}12.0 \\
13.5 \\
14.0\end{array}$ & $\begin{array}{l}12.0 \\
13.5 \\
20.0\end{array}$ & $\begin{array}{l}\text { lead } \\
\text { lead } \\
304 s s t\end{array}$ & $\begin{array}{l}\text { lead } \\
\text { lead } \\
304 s s t\end{array}$ & $\begin{array}{l}0.0 \\
0.0 \\
0.0\end{array}$ & $\begin{array}{l}\text { none } \\
\text { none } \\
\text { sand-b }\end{array}$ \\
\hline E.2 & $\begin{array}{l}\text { stab } \\
\text { stab } \\
\operatorname{can}\end{array}$ & $\begin{array}{l}12.0 \\
12.0 \\
13.5\end{array}$ & $\begin{array}{l}12.0 \\
12.0 \\
14.0\end{array}$ & $\left|\begin{array}{l}12.0 \\
12.0 \\
14.0\end{array}\right|$ & $\begin{array}{l}12.0 \\
13.5 \\
14.2\end{array}$ & $\begin{array}{l}12.0 \\
13.5 \\
20.0\end{array}$ & $\begin{array}{l}\text { lead } \\
\text { lead } \\
\text { steel }\end{array}$ & $\begin{array}{l}\text { lead } \\
\text { lead } \\
\text { zire }\end{array}$ & $\begin{array}{l}0.0 \\
0.0 \\
0.0\end{array}$ & $\begin{array}{l}\text { none } \\
\text { none } \\
\text { sand-b }\end{array}$ \\
\hline
\end{tabular}


Table 4-1. (Continued)

\begin{tabular}{|c|c|c|c|c|c|c|c|c|c|c|}
\hline \multirow[t]{2}{*}{ Concept } & \multirow[t]{2}{*}{ Element } & \multicolumn{2}{|c|}{$\begin{array}{l}\text { Inside } \\
\text { ilaterial }\end{array}$} & \multicolumn{2}{|c|}{$\begin{array}{l}\text { Outside } \\
\text { Material }\end{array}$} & \multirow{2}{*}{$\begin{array}{c}\text { Filler } \\
00\end{array}$} & \multirow[t]{2}{*}{$\begin{array}{l}\text { Inside } \\
\text { Materia }\end{array}$} & \multirow[t]{2}{*}{$\begin{array}{l}\text { Outside } \\
\text { Material }\end{array}$} & \multirow{2}{*}{$\begin{array}{c}\text { Coating } \\
\text { Delay } \\
\text { (yrs) }\end{array}$} & \multirow[t]{2}{*}{ Filler } \\
\hline & & ID & 100 & 10 & 00 & & & & & \\
\hline E.3 & $\begin{array}{l}\text { stab } \\
\text { stab } \\
\text { can }\end{array}$ & $\begin{array}{l}12.0 \\
12.0 \\
13.5\end{array}$ & $\begin{array}{l}12.0 \\
12.0 \\
14.0\end{array}$ & $\begin{array}{l}12.0 \\
12.0 \\
14.0\end{array}$ & $\begin{array}{l}12.0 \\
13.5 \\
14.2\end{array}$ & $\begin{array}{l}12.0 \\
13.5 \\
20.0\end{array}$ & $\begin{array}{l}\text { lead } \\
\text { lead } \\
3045 s t\end{array}$ & $\begin{array}{l}\text { lead } \\
\text { lead } \\
\text { zire }\end{array}$ & $\begin{array}{l}0.0 \\
0.0 \\
0.0\end{array}$ & $\begin{array}{l}\text { none } \\
\text { none } \\
\text { sand-b }\end{array}$ \\
\hline BASE & $\begin{array}{l}\text { stab } \\
\text { can }\end{array}$ & $\begin{array}{l}12.0 \\
13.5\end{array}$ & $\begin{array}{l}13.5 \\
13.6\end{array}$ & $\begin{array}{l}13.5 \\
13.6\end{array}$ & $\begin{array}{l}13.5 \\
13.6\end{array}$ & $\begin{array}{l}13.5 \\
13.6\end{array}$ & $\begin{array}{l}\text { helium } \\
\text { steel }\end{array}$ & $\begin{array}{l}\text { helium } \\
\text { steel }\end{array}$ & $\begin{array}{l}0.0 \\
0.0\end{array}$ & $\begin{array}{l}\text { none } \\
\text { none }\end{array}$ \\
\hline
\end{tabular}


Table 4-2. Summary of Concepts Studied in FY'80 Follow-on Work Using Previous Version of "BARIER" Code.

(Stula, T980a).

\begin{tabular}{|c|c|c|c|c|c|c|c|c|c|c|}
\hline Comept & EI줄 & 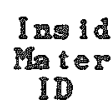 & OD & $\begin{array}{c}01 \\
10 \\
15\end{array}$ & $\begin{array}{l}1 d \mathrm{~d} \\
\text { I } 1 \\
\text { OD }\end{array}$ & $\begin{array}{c}\text { F11E } \\
\text { OD }\end{array}$ & $\begin{array}{c}\text { Inside } \\
\text { Mestal }\end{array}$ & $\begin{array}{l}\text { Outgate } \\
\text { Mateid }\end{array}$ & $\begin{array}{c}\text { Coat ing } \\
\text { Delay } \\
(y z d)\end{array}$ & FIler \\
\hline A. 11 & $\frac{\cos }{\mathrm{cas}}$ & $\begin{array}{l}12.0 \\
13.5\end{array}$ & $\begin{array}{l}13.5 \\
14.0\end{array}$ & $\begin{array}{l}13.5 \\
14.0\end{array}$ & $\begin{array}{l}13.5 \\
14.0\end{array}$ & $\begin{array}{l}13.5 \\
40.0\end{array}$ & 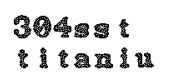 & $\begin{array}{l}30491 \\
11021\end{array}$ & 0.0 & $\begin{array}{l}\text { mone } \\
\text { and- }\end{array}$ \\
\hline A. 12 & $\begin{array}{l}c a b \\
c a b\end{array}$ & $\begin{array}{l}12.0 \\
13.5\end{array}$ & $\begin{array}{l}13.5 \\
14.0\end{array}$ & $\begin{array}{l}13.5 \\
14.9\end{array}$ & $\begin{array}{l}13.5 \\
14.0\end{array}$ & $\begin{array}{l}13.3 \\
40.0\end{array}$ & $\begin{array}{l}3045 s t \\
c a-21\end{array}$ & $\begin{array}{l}304091 \\
\text { cus- }\end{array}$ & 0.0 & $\begin{array}{l}\text { none } \\
\text { ad-b }\end{array}$ \\
\hline D1. 12 & 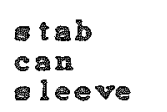 & $\begin{array}{l}12.9 \\
13.5 \\
14.5\end{array}$ & $\begin{array}{l}13.5 \\
13.0 \\
22.5\end{array}$ & $\begin{array}{l}13.5 \\
14.5 \\
22.5\end{array}$ & $\begin{array}{l}13.5 \\
14.0 \\
22.6\end{array}$ & $\begin{array}{l}13.5 \\
14.5 \\
48.0\end{array}$ & $\begin{array}{l}\text { teel } \\
\text { tee } \\
\text { lyon }\end{array}$ & $\begin{array}{l}\text { Ded } \\
\text { steel } \\
\text { z lac }\end{array}$ & $\begin{array}{l}0.0 \\
0.0 \\
0.0\end{array}$ & $\begin{array}{l}\text { ane } \\
\text { and-b } \\
\text { gad-b }\end{array}$ \\
\hline B1.13 & $\begin{array}{l}\text { cab } \\
\text { levere }\end{array}$ & $\begin{array}{l}12.9 \\
13.5 \\
19.5\end{array}$ & $\begin{array}{l}13.5 \\
14.0 \\
22.5\end{array}$ & $\begin{array}{l}13.5 \\
1.06 \\
22.5\end{array}$ & $\begin{array}{l}13.5 \\
14.0 \\
22.8\end{array}$ & $\begin{array}{l}13.5 \\
14.5 \\
48.0\end{array}$ & $\begin{array}{l}\text { teel } \\
\text { teel } \\
\text { lron }\end{array}$ & $\begin{array}{l}\text { teel } \\
\text { teel } \\
\text { zide }\end{array}$ & $\begin{array}{l}0.0 \\
0.0 \\
0.0\end{array}$ & $\begin{array}{l}\text { none } \\
\text { and-b } \\
\text { and-b }\end{array}$ \\
\hline 81. & $\begin{array}{l}\text { tab } \\
\text { can } \\
100\end{array}$ & $\begin{array}{l}13.0 \\
13.5 \\
14.5\end{array}$ & $\begin{array}{l}13.5 \\
14.0 \\
22.5\end{array}$ & $\begin{array}{l}13.5 \\
14.0 \\
23.5\end{array}$ & $\begin{array}{l}13.5 \\
14.0 \\
23.0\end{array}$ & $\begin{array}{l}13.5 \\
14.5 \\
48.0\end{array}$ & $\begin{array}{l}\text { teel } \\
\text { teel } \\
\text { tod }\end{array}$ & $\begin{array}{l}\text { iteel } \\
\text { iteel } \\
\text { atre }\end{array}$ & $\begin{array}{l}0.0 \\
0.0 \\
0.0\end{array}$ & $\begin{array}{l}\text { yone } \\
\text { and-b } \\
\text { and-b }\end{array}$ \\
\hline B1. 15 & $\begin{array}{l}\text { cab } \\
\text { can } \\
\text { lecve }\end{array}$ & $\begin{array}{l}12.9 \\
13.5 \\
14.5\end{array}$ & $\begin{array}{l}13.5 \\
13.0 \\
22.5\end{array}$ & $\begin{array}{l}13.5 \\
14.0 \\
22.5\end{array}$ & $\begin{array}{l}13.5 \\
1.40 \\
22.7\end{array}$ & $\begin{array}{l}13.5 \\
14.5 \\
48.0\end{array}$ & $\begin{array}{l}\text { teel } \\
\text { teel } \\
3045 \text { t }\end{array}$ & $\begin{array}{l}\text { geel } \\
\text { zteel } \\
\text { zlre }\end{array}$ & $\begin{array}{l}0.0 \\
0.0 \\
0.0\end{array}$ & $\begin{array}{l}\text { none } \\
\text { sand-b } \\
\text { and-b }\end{array}$ \\
\hline B1. 16 & $\begin{array}{l}\text { cab } \\
\text { cateeve }\end{array}$ & $\begin{array}{l}12.9 \\
13.9 \\
14.9\end{array}$ & $\begin{array}{l}13.5 \\
14.0 \\
22.5\end{array}$ & $\begin{array}{l}13.5 \\
14.9 \\
22.5\end{array}$ & $\begin{array}{l}13.5 \\
14.0 \\
22.6\end{array}$ & $\begin{array}{l}13.5 \\
14.5 \\
48.0\end{array}$ & 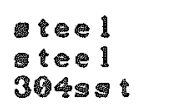 & $\begin{array}{l}\text { ted } \\
\text { ted } \\
218 c\end{array}$ & $\begin{array}{l}.0 \\
0.0\end{array}$ & $\begin{array}{l}\text { gove } \\
\text { a ad-b } \\
\text { acd-b }\end{array}$ \\
\hline B1. 18 & $\begin{array}{l}\text { cab } \\
\text { can } \\
\text { leeve }\end{array}$ & $\begin{array}{l}12.0 \\
13.5 \\
14.5\end{array}$ & $\begin{array}{l}13.5 \\
14.0 \\
22.5\end{array}$ & $\begin{array}{l}13.5 \\
14.0 \\
25.9\end{array}$ & $\begin{array}{l}13.5 \\
14.0 \\
23.0\end{array}$ & $\begin{array}{l}13.5 \\
14.5 \\
48.0\end{array}$ & $\begin{array}{l}\text { teel } \\
\text { seel } \\
3946 \text { i }\end{array}$ & $\begin{array}{l}\text { tee } \\
\text { tee l } \\
\text { Ixce }\end{array}$ & $\begin{array}{l}0.0 \\
0.0 \\
0.0\end{array}$ & $\begin{array}{l}\text { mone } \\
\text { and-b } \\
\text { ad-b }\end{array}$ \\
\hline 81.18 & : & 12. & 13.5 & 13.5 & 13.5 & 13.5 & tee 1 & $t \in \operatorname{lt}$ & 0.0 & zone \\
\hline
\end{tabular}


Table 4-2. Summary of Concepts Studied in FY'80 Follow-on Work Using Previous Version of "BARIER" Code. (Stula, 1980a). (Continued)

\begin{tabular}{|c|c|c|c|c|c|c|c|c|c|c|}
\hline Concopt & 2 1 중 & 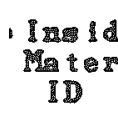 & OD & $\begin{array}{c}0 \% \\
\text { 14 } \\
11\end{array}$ & $\begin{array}{l}110 \\
121 \\
\text { OD }\end{array}$ & $\begin{array}{c}1120 \\
0 D\end{array}$ & 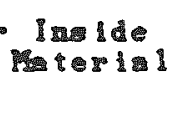 & 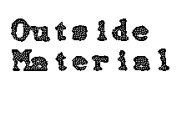 & 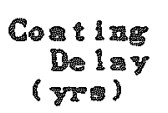 & Filler \\
\hline 81.18 & $\begin{array}{l}\text { exa } \\
\text { loeve }\end{array}$ & $\begin{array}{l}13.5 \\
14.5\end{array}$ & $\begin{array}{l}14.0 \\
22.5\end{array}$ & $\begin{array}{l}14.0 \\
22.5\end{array}$ & $\begin{array}{l}14.0 \\
32.7\end{array}$ & $\begin{array}{l}1.5 \\
48.5\end{array}$ & $\begin{array}{l}\text { toe. } \\
\text { l+on }\end{array}$ & $\begin{array}{l}\text { tee } 1 \\
304 \text { 날 }\end{array}$ & $\begin{array}{l}0 \\
0.0\end{array}$ & $\begin{array}{l}a^{-b}-b \\
\text { ard-b }\end{array}$ \\
\hline B1.19 & $\begin{array}{l}\text { cab } \\
\text { can } \\
\text { leeve }\end{array}$ & $\begin{array}{l}12.0 \\
13.5 \\
14.5\end{array}$ & $\begin{array}{l}13.5 \\
14.0 \\
22.5\end{array}$ & $\begin{array}{l}13.5 \\
14.0 \\
22.5\end{array}$ & $\begin{array}{l}13.5 \\
14.0 \\
23.0\end{array}$ & $\begin{array}{l}13.5 \\
14.5 \\
48.0\end{array}$ & 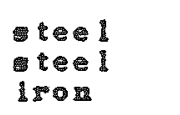 & $\begin{array}{l}\text { tee } 1 \\
\text { see } 1 \\
30459\end{array}$ & $\begin{array}{l}0.0 \\
0.9 \\
0.0\end{array}$ & $\begin{array}{l}\text { noxe } \\
\operatorname{sand}-b \\
\operatorname{sind}-b\end{array}$ \\
\hline 81.20 & $\begin{array}{l}\text { tab } \\
\text { can } \\
\text { leve }\end{array}$ & $\begin{array}{l}12.0 \\
13.5 \\
14.5\end{array}$ & $\begin{array}{l}13.5 \\
14.0 \\
18.5\end{array}$ & $\begin{array}{l}13.2 \\
14.9 \\
19.5\end{array}$ & $\begin{array}{l}13.5 \\
14.0 \\
18.7\end{array}$ & $\begin{array}{l}13.5 \\
14.5 \\
43.0\end{array}$ & $\begin{array}{l}\text { teel } \\
\text { tree } \\
\text { tron }\end{array}$ & 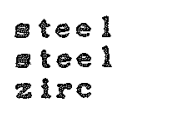 & $\begin{array}{l}0.0 \\
0.0 \\
0.0\end{array}$ & $\begin{array}{l}\text { none } \\
\text { and-b } \\
\text { and-b }\end{array}$ \\
\hline B1.21 & $\begin{array}{l}\text { cab } \\
\text { can } \\
100\end{array}$ & $\begin{array}{l}12.0 \\
13.5 \\
1.5\end{array}$ & $\begin{array}{l}13.5 \\
14.0 \\
13.5\end{array}$ & $\begin{array}{l}13.5 \\
14.0 \\
12.5\end{array}$ & $\begin{array}{l}13.5 \\
14.0 \\
19.0\end{array}$ & $\begin{array}{l}13.5 \\
1.5 \\
48.5\end{array}$ & 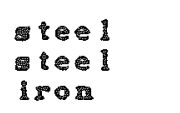 & 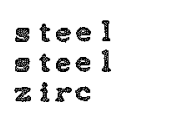 & $\begin{array}{l}0.0 \\
0.0 \\
0.0\end{array}$ & $\begin{array}{l}\text { aode } \\
\text { acd-b } \\
\text { ad-b }\end{array}$ \\
\hline 1.22 & $\begin{array}{l}\text { tab } \\
\operatorname{can} \\
\text { lieve }\end{array}$ & $\begin{array}{l}12.0 \\
13.5 \\
14.5\end{array}$ & $\begin{array}{l}13.5 \\
14.0 \\
15.0\end{array}$ & $\begin{array}{l}13.5 \\
14.0 \\
15.0\end{array}$ & $\begin{array}{l}13.5 \\
14.9 \\
15.1\end{array}$ & $\begin{array}{l}13.5 \\
14.5 \\
48.0\end{array}$ & 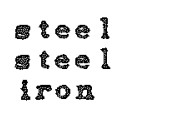 & $\begin{array}{l}\text { teel } \\
\text { teel } \\
\text { z } 150\end{array}$ & $\begin{array}{l}0.0 \\
0.0 \\
0.0\end{array}$ & $\begin{array}{l}\text { noae } \\
\text { and-b } \\
\text { and-b }\end{array}$ \\
\hline 81.23 & $\begin{array}{l}\text { tab } \\
\text { ea } \\
\text { leeve }\end{array}$ & $\begin{array}{l}12.0 \\
13.5 \\
14.5\end{array}$ & $\begin{array}{l}13.5 \\
14.0 \\
15.0\end{array}$ & $\begin{array}{l}13.9 \\
14.9 \\
15.0\end{array}$ & $\begin{array}{l}13.5 \\
14.0 \\
15.1\end{array}$ & $\begin{array}{l}13.3 \\
14.5 \\
48.0\end{array}$ & $\begin{array}{l}\text { teel } \\
\text { teel } \\
\text { frot }\end{array}$ & $\begin{array}{l}\text { teel } \\
\text { tee I } \\
3045 \text { t }\end{array}$ & $\begin{array}{l}.0 \\
0.0 \\
0.0\end{array}$ & $\begin{array}{l}\text { none } \\
\text { and-b } \\
\text { and-b }\end{array}$ \\
\hline 1.24 & $\begin{array}{l}\text { tab } \\
\text { can } \\
\text { leve }\end{array}$ & $\begin{array}{l}12.0 \\
13.5 \\
14.5\end{array}$ & $\begin{array}{l}13.5 \\
14.0 \\
18.5\end{array}$ & $\begin{array}{l}13.5 \\
14.0 \\
18.5\end{array}$ & $\begin{array}{l}13.5 \\
13.0 \\
18.5\end{array}$ & $\begin{array}{l}13.5 \\
14.5 \\
40.0\end{array}$ & $\begin{array}{l}\text { ted } \\
\text { tee } 1 \\
3045 \text { t }\end{array}$ & $\begin{array}{l}\text { teel } \\
\text { iteel } \\
30458 \text { i }\end{array}$ & $\begin{array}{l}0.0 \\
0.0 \\
0.0\end{array}$ & $\begin{array}{l}\text { none } \\
\text { sand-b } \\
\text { sand-b }\end{array}$ \\
\hline 81.25 & $\begin{array}{l}\text { cab } \\
\text { can leeve }\end{array}$ & $\begin{array}{l}12.0 \\
13.5 \\
14.5\end{array}$ & $\begin{array}{l}13.5 \\
14.9 \\
21.5\end{array}$ & $\begin{array}{r}13.5 \\
1.96 \\
21.9\end{array}$ & $\begin{array}{l}13.5 \\
14.0 \\
21.5\end{array}$ & $\begin{array}{l}13.5 \\
14.5 \\
48.0\end{array}$ & $\begin{array}{l}\text { teel } \\
\text { teel } \\
\text { coppes }\end{array}$ & $\begin{array}{l}\text { teel } \\
\text { ceel } \\
\text { copper }\end{array}$ & $\begin{array}{l}.0 \\
0.0 \\
0.0\end{array}$ & $\begin{array}{l}\text { uone } \\
\text { and-b } \\
\text { and-b }\end{array}$ \\
\hline 1.26 & $\begin{array}{l}a b \\
c a b \\
1<0\end{array}$ & $\begin{array}{l}12.0 \\
13.5 \\
1.53\end{array}$ & $\begin{array}{l}13.5 \\
11.0 \\
21.5\end{array}$ & $\begin{array}{l}13.5 \\
13.5 \\
21.5\end{array}$ & $\begin{array}{l}13.5 \\
14.0 \\
21.5\end{array}$ & $\begin{array}{l}13.5 \\
13.5 \\
48.0\end{array}$ & $\begin{array}{l}\text { teel } \\
\text { teel } \\
\text { Ixom } 1\end{array}$ & $\begin{array}{l}\text { teel } \\
\text { teel } \\
\text { lacol }\end{array}$ & $\begin{array}{l}0.0 \\
0.0 \\
0.0\end{array}$ & $\begin{array}{l}\text { none } \\
\text { and-b } \\
\text { ad-b }\end{array}$ \\
\hline
\end{tabular}




\section{Table 4-2. Summary of Concepts Studied in FY' 80 Follow-on Work Using Previous Version of "BARIER" Code. (Stuia, 1980a). (Continued)}

\begin{tabular}{|c|c|c|c|c|c|c|c|c|c|c|}
\hline Coresp & ELe & $\begin{array}{l}\text { Ias Id } \\
\text { Mater } \\
\text { ID }\end{array}$ & id 1 & $\begin{array}{l}\text { Ont } \\
\text { Mate } \\
\text { ID }\end{array}$ & $\begin{array}{l}1 d e \\
\text { Ia } 1 \\
\text { OD }\end{array}$ & $\begin{array}{c}\text { MLE } \\
\text { OD }\end{array}$ & 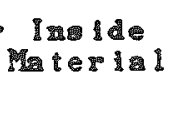 & 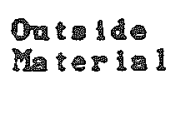 & 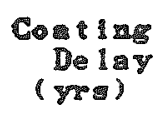 & F1110 \\
\hline B1.28 & $\begin{array}{l}\text { exb } \\
\text { can } \\
\text { lecter }\end{array}$ & $\begin{array}{l}12.0 \\
13.5 \\
14.5\end{array}$ & $\begin{array}{l}13.5 \\
14.9 \\
21.5\end{array}$ & $\begin{array}{l}13.5 \\
14.9 \\
21.5\end{array}$ & $\begin{array}{l}13.5 \\
14.0 \\
21.5\end{array}$ & $\begin{array}{l}13.5 \\
1.5 \\
48.0\end{array}$ & $\begin{array}{l}\text { tee } 1 \\
\text { tee } 1 \\
30453\end{array}$ & $\begin{array}{l}\text { tee } 1 \\
\text { tee } 1 \\
304=0\end{array}$ & $\begin{array}{l}.0 \\
0.0 \\
.0\end{array}$ & $\begin{array}{l}\text { nase } \\
\text { and-b } \\
\text { ad-b }\end{array}$ \\
\hline 81.28 & $\begin{array}{l}\text { tab } \\
\text { eara } \\
\text { leeve }\end{array}$ & $\begin{array}{l}12.0 \\
13.5 \\
14.5\end{array}$ & $\begin{array}{l}13.5 \\
14.0 \\
15.0\end{array}$ & $\begin{array}{l}13.5 \\
14.9 \\
15.9\end{array}$ & $\begin{array}{l}13.5 \\
14.0 \\
15.0\end{array}$ & $\begin{array}{l}13.5 \\
13.5 \\
90.0\end{array}$ & $\begin{array}{l}\text { tee } 1 \\
\text { teel } \\
\text { copper }\end{array}$ & $\begin{array}{l}\text { steel } \\
\text { teel } \\
\text { copper }\end{array}$ & $\begin{array}{l}0.9 \\
0.6 \\
0.6\end{array}$ & $\begin{array}{l}\text { none } \\
\text { and-b } \\
\text { gand-b }\end{array}$ \\
\hline B1. 29 & $\begin{array}{l}\text { iab } \\
\text { cat } \\
\text { leve }\end{array}$ & $\begin{array}{l}12.0 \\
13.5 \\
104.5\end{array}$ & $\begin{array}{l}13.5 \\
13.0 \\
15.0\end{array}$ & $\begin{array}{l}13.5 \\
14.0 \\
13.0\end{array}$ & $\begin{array}{l}13.5 \\
14.0 \\
15.0\end{array}$ & $\begin{array}{l}13.5 \\
14.5 \\
40.6\end{array}$ & $\begin{array}{l}\text { teel } \\
\text { incel } 1 \\
\text { ineore } 1\end{array}$ & $\begin{array}{l}\text { teel } \\
\text { teel } \\
\text { neorel }\end{array}$ & $\begin{array}{l}0.0 \\
0.0 \\
0.8\end{array}$ & $\begin{array}{l}\text { aone } \\
\text { and-b } \\
\text { ad-b }\end{array}$ \\
\hline 81.30 & $\begin{array}{l}\text { id } \\
\text { cain } \\
\text { levere }\end{array}$ & $\begin{array}{l}12.0 \\
18.5 \\
16.5\end{array}$ & $\begin{array}{l}13.75 \\
14.0 \\
15.0\end{array}$ & $\begin{array}{l}13.5 \\
14.0 \\
15.0\end{array}$ & $\begin{array}{l}13.5 \\
14.0 \\
15.0\end{array}$ & $\begin{array}{l}13.5 \\
16.5 \\
40.0\end{array}$ & 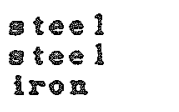 & 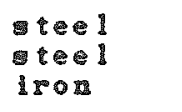 & $\begin{array}{l}0.0 \\
0.0 \\
0.0\end{array}$ & $\begin{array}{l}\text { none } \\
\text { sad-b } \\
\text { sad }-b\end{array}$ \\
\hline 81.31 & $\begin{array}{l}\text { 대 } \\
\text { can } \\
\text { leeva }\end{array}$ & $\begin{array}{l}12.0 \\
13.5 \\
14.5\end{array}$ & $\begin{array}{l}13.5 \\
14.9 \\
26.5\end{array}$ & $\begin{array}{l}13.5 \\
14.0 \\
26.5\end{array}$ & $\begin{array}{l}13.5 \\
14.0 \\
26.5\end{array}$ & $\begin{array}{l}13.5 \\
13.5 \\
48.0\end{array}$ & $\begin{array}{l}\text { teel } \\
\text { tee } 1 \\
3045: 1\end{array}$ & $\begin{array}{l}\text { teel } \\
\text { teel } \\
30435\end{array}$ & $\begin{array}{l}0.0 \\
0.0 \\
0.0\end{array}$ & $\begin{array}{l}\text { nome } \\
\text { sand-b } \\
\text { sand-b }\end{array}$ \\
\hline 81.32 & $\begin{array}{l}\text { tab } \\
\text { can } \\
\text { leve }\end{array}$ & $\begin{array}{l}12.0 \\
13.5 \\
14.5\end{array}$ & $\begin{array}{l}13.5 \\
14.0 \\
20.5\end{array}$ & $\begin{array}{l}13.5 \\
14.96 \\
20.9\end{array}$ & $\begin{array}{l}13.5 \\
14.0 \\
21.0\end{array}$ & $\begin{array}{l}13.5 \\
14.5 \\
48.5\end{array}$ & 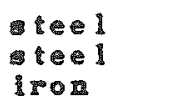 & 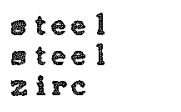 & $\begin{array}{l}0.0 \\
0.0 \\
0.0\end{array}$ & $\begin{array}{l}\text { none } \\
\text { sand-b } \\
\text { and-b }\end{array}$ \\
\hline B1. 33 & $\begin{array}{l}\text { lab } \\
\text { ea } \\
\text { lecve }\end{array}$ & $\begin{array}{l}12.0 \\
13.5 \\
16.5\end{array}$ & $\begin{array}{l}13.5 \\
14.0 \\
20.5\end{array}$ & $\begin{array}{l}13.5 \\
14.0 \\
20.5\end{array}$ & $\begin{array}{l}13.5 \\
14.0 \\
20.7\end{array}$ & $\begin{array}{l}13.5 \\
14.5 \\
48.0\end{array}$ & $\begin{array}{l}\text { tee } 1 \\
\text { tee } 1 \\
\text { Lon }\end{array}$ & $\begin{array}{l}\text { teel } \\
\text { teel } \\
\text { zire }\end{array}$ & $\begin{array}{l}0.0 \\
0.0 \\
0.9\end{array}$ & $\begin{array}{l}\text { none } \\
\text { sad-b } \\
\text { sand-b }\end{array}$ \\
\hline 31.34 & $\begin{array}{l}\text { tab } \\
\text { can } \\
\text { leeve }\end{array}$ & $\begin{array}{l}12.5 \\
13.5 \\
14.5\end{array}$ & $\begin{array}{l}13.5 \\
14.0 \\
26.5\end{array}$ & $\begin{array}{l}13.5 \\
14.0 \\
26.5\end{array}$ & $\begin{array}{l}13.5 \\
14.0 \\
26.5\end{array}$ & $\begin{array}{l}13.5 \\
14.5 \\
48.0\end{array}$ & $\begin{array}{l}\text { teel } \\
\text { ited } \\
\text { tron }\end{array}$ & $\begin{array}{l}\text { Steel } \\
\text { treel } \\
\text { lrot }\end{array}$ & $\begin{array}{l}0.0 \\
0.0 \\
0.0\end{array}$ & $\begin{array}{l}\text { mone } \\
\text { gad-b } \\
\text { sad-b }\end{array}$ \\
\hline B1.37 & 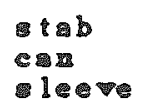 & $\begin{array}{l}12.0 \\
13.5 \\
14.5\end{array}$ & $\begin{array}{l}13.5 \\
14.0 \\
26.5\end{array}$ & $\begin{array}{l}13.5 \\
13.0 \\
26.5\end{array}$ & $\begin{array}{l}13.5 \\
14.0 \\
27.0\end{array}$ & $\begin{array}{l}13.5 \\
14.5 \\
40.0\end{array}$ & $\begin{array}{l}\text { gteel } \\
\text { teel } \\
304 \text { \& }\end{array}$ & $\begin{array}{l}\text { Dteel } \\
\text { teel } \\
\text { zirc }\end{array}$ & $\begin{array}{l}0.0 \\
0.0 \\
0.0\end{array}$ & $\begin{array}{l}\text { none } \\
\text { sand-b } \\
\text { and-b }\end{array}$ \\
\hline
\end{tabular}


Table 4-2. Summary of Concepts Studied in Fy'80 Follow-on Work Using Previous-Version of "BARIER" Code. (Stula, 1980a). (Continued)

\begin{tabular}{|c|c|c|c|c|c|c|c|c|c|c|}
\hline Corepe & 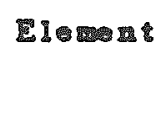 & 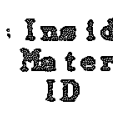 & 1. & 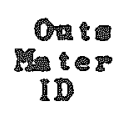 & $\begin{array}{l}\text { Id } \\
\text { OD } \\
\text { OD }\end{array}$ & $\begin{array}{c}\text { 11128 } \\
00\end{array}$ & 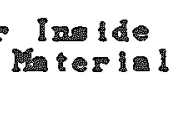 & 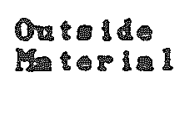 & 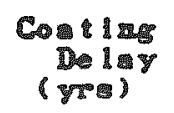 & S1105 \\
\hline B1. 36 & $\begin{array}{l}\text { ed } \\
\text { ean } \\
\text { leve }\end{array}$ & $\begin{array}{l}12.0 \\
13.5 \\
14.5\end{array}$ & $\begin{array}{l}13.5 \\
14.9 \\
26.5\end{array}$ & $\begin{array}{l}13.5 \\
1.60 \\
26.5\end{array}$ & $\begin{array}{l}13.5 \\
1.96 \\
26.6\end{array}$ & $\begin{array}{l}3.9 \\
18.9 \\
48.9\end{array}$ & 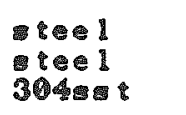 & 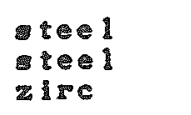 & $\begin{array}{l}0.9 \\
0.0 \\
0.0\end{array}$ & $\begin{array}{l}\text { none } \\
\text { and ab } \\
\text { and-b }\end{array}$ \\
\hline 2. & 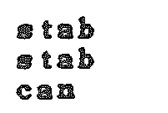 & $\begin{array}{l}12.0 \\
12.0 \\
13.5\end{array}$ & $\begin{array}{l}12.0 \\
12.0 \\
14.0\end{array}$ & $\begin{array}{l}12.0 \\
12.0 \\
14.6\end{array}$ & $\begin{array}{l}12.0 \\
13.9 \\
1.9\end{array}$ & $\begin{array}{l}12.0 \\
13.5 \\
70.0\end{array}$ & $\begin{array}{l}\text { lead } \\
\text { lead } \\
\text { zirc }\end{array}$ & $\begin{array}{l}1 \cos \\
1 \in d \\
z 1=6\end{array}$ & $\begin{array}{l}0.9 \\
0.0\end{array}$ & $\begin{array}{l}\text { gone } \\
\text { mone } \\
\text { ad-b }\end{array}$ \\
\hline E. & $\begin{array}{l}\text { tab } \\
\text { cab } \\
\text { cal }\end{array}$ & $\begin{array}{l}12.0 \\
12.0 \\
13.3\end{array}$ & $\begin{array}{l}12.0 \\
12.0 \\
14.0\end{array}$ & $\begin{array}{l}12.6 \\
12.0 \\
1.6\end{array}$ & $\begin{array}{l}12.0 \\
13.9 \\
14.0\end{array}$ & $\begin{array}{r}12.0 \\
13.5 \\
26.0\end{array}$ & $\begin{array}{l}\text { lead } \\
\text { lead } \\
\text { lncome } 1\end{array}$ & $\begin{array}{l}\text { led } \\
\text { led } \\
\text { laconel }\end{array}$ & $\begin{array}{l}0.0 \\
0.0 \\
0.0\end{array}$ & $\begin{array}{l}\text { gone } \\
\text { moae } \\
\text { and-b }\end{array}$ \\
\hline 雷. 6 & 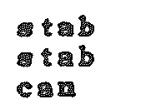 & $\begin{array}{l}12.0 \\
12.0 \\
13.5\end{array}$ & $\begin{array}{l}12.0 \\
12.0 \\
14.0\end{array}$ & $\begin{array}{l}12.0 \\
12.0 \\
14.9\end{array}$ & $\begin{array}{l}12.9 \\
13.9 \\
14.9\end{array}$ & $\begin{array}{l}12.0 \\
13.5 \\
20.6\end{array}$ & $\begin{array}{l}\text { led } \\
\text { lead } \\
\text { tee }\end{array}$ & $\begin{array}{l}\text { led } \\
\text { led } \\
\text { iee }\end{array}$ & $\begin{array}{l}0.0 \\
0.0 \\
0.0\end{array}$ & $\begin{array}{l}\text { none } \\
\text { mone } \\
\text { ad-b }\end{array}$ \\
\hline E. & 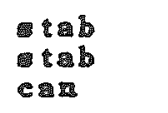 & $\begin{array}{l}12.0 \\
12.0 \\
13.5\end{array}$ & $\begin{array}{l}12.0 \\
12.0 \\
14.0\end{array}$ & $\begin{array}{l}12.0 \\
12.0 \\
14.0\end{array}$ & $\begin{array}{l}12.0 \\
13.5 \\
14.0\end{array}$ & $\begin{array}{l}12.0 \\
13.5 \\
20.6\end{array}$ & $\begin{array}{l}3045 \sqrt{1} \\
30461 \\
304891\end{array}$ & $\begin{array}{l}30438 \\
30438 \\
30439\end{array}$ & $\begin{array}{l}0.0 \\
0.0 \\
0.9\end{array}$ & $\begin{array}{l}\text { none } \\
\text { no ad } \\
\text { ad ab }\end{array}$ \\
\hline 宦。8 & 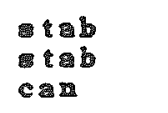 & $\begin{array}{l}12.0 \\
12.0 \\
13.7\end{array}$ & $\begin{array}{l}12.0 \\
12.0 \\
14.0\end{array}$ & $\begin{array}{l}12.0 \\
12.0 \\
14.0\end{array}$ & $\begin{array}{l}12.0 \\
13.5 \\
14.0\end{array}$ & $\begin{array}{l}12.0 \\
13.5 \\
30.0\end{array}$ & $\begin{array}{l}\text { teel } \\
\text { teel } \\
\text { teel }\end{array}$ & $\begin{array}{l}\text { teel } \\
\text { teel } \\
\text { teel }\end{array}$ & $\begin{array}{l}0.0 \\
0.0 \\
0.0\end{array}$ & $\begin{array}{l}\text { gone } \\
\text { none } \\
\text { and-b }\end{array}$ \\
\hline E.9 & 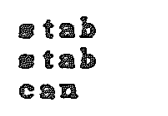 & $\begin{array}{l}12.0 \\
12.0 \\
13.5\end{array}$ & $\begin{array}{l}12.9 \\
12.9 \\
16.0\end{array}$ & $\begin{array}{l}12.0 \\
12.0 \\
1.0\end{array}$ & $\begin{array}{l}12.0 \\
13.9 \\
14.0\end{array}$ & $\begin{array}{l}12.0 \\
13.5 \\
20.0\end{array}$ & $\begin{array}{l}\text { aconel } \\
\text { meonel } \\
\text { meonel }\end{array}$ & $\begin{array}{l}\text { aconel } \\
\text { acone I } \\
\text { acone } 1\end{array}$ & $\begin{array}{l}0.0 \\
0.0 \\
0.0\end{array}$ & $\begin{array}{l}\text { none } \\
\text { none } \\
\text { and-b }\end{array}$ \\
\hline 瓷. 10 & 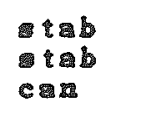 & $\begin{array}{l}12.0 \\
12.0 \\
13.5\end{array}$ & $\begin{array}{l}12.0 \\
12.0 \\
15.5\end{array}$ & $\begin{array}{l}12.0 \\
12.0 \\
15.9\end{array}$ & $\begin{array}{l}12.0 \\
13.5 \\
15.5\end{array}$ & $\begin{array}{l}12.0 \\
13.0 \\
20.0\end{array}$ & $\begin{array}{l}\text { lead } \\
\text { lead } \\
3045 \text { is }\end{array}$ & $\begin{array}{l}\text { lead } \\
\text { lead } \\
30456 \text { t }\end{array}$ & $\begin{array}{l}0.0 \\
0.0 \\
0.0\end{array}$ & $\begin{array}{l}\text { none } \\
\text { none } \\
\text { bad-b }\end{array}$ \\
\hline E. 11 & 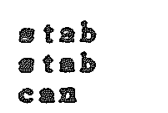 & $\begin{array}{l}12.0 \\
12.0 \\
13 .\end{array}$ & $\begin{array}{l}12.0 \\
12.0 \\
15.5\end{array}$ & $\begin{array}{l}12.0 \\
12.0 \\
1.5\end{array}$ & $\begin{array}{l}12.0 \\
13.5 \\
1.5\end{array}$ & $\begin{array}{l}12.0 \\
13.5 \\
20.0\end{array}$ & 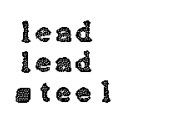 & $\begin{array}{l}\text { led } \\
1: d \\
\text { ide } 1\end{array}$ & $\begin{array}{l}.0 \\
0.0 \\
0.0\end{array}$ & $\begin{array}{l}\text { none } \\
\text { none } \\
\text { ad-b }\end{array}$ \\
\hline
\end{tabular}


Table 4-2. Sumnary of Concepts Studied in FY'80 Follow-on Work Using Previous Version of "BARIER" Code.

(stula, 1980a). (Continued)

\begin{tabular}{|c|c|c|c|c|c|c|c|c|c|c|}
\hline Comept & Eloat & 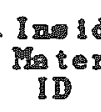 & $\frac{19}{01}$ & 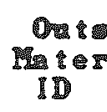 & $\begin{array}{l}21 \\
01 \\
01\end{array}$ & $\begin{array}{c}\text { 1 } 18 \\
\text { OD }\end{array}$ & 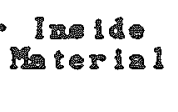 & 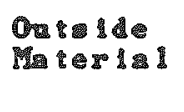 & 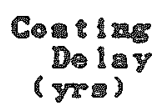 & P110r \\
\hline E. 12 & $\begin{array}{l}\text { 1ab } \\
\operatorname{cab} \\
\operatorname{cac}\end{array}$ & $\begin{array}{l}12.0 \\
12.0 \\
13 .\end{array}$ & $\begin{array}{l}12.0 \\
12.0 \\
1.40\end{array}$ & $\begin{array}{l}12.0 \\
12.0 \\
14.0\end{array}$ & $\begin{array}{l}12.0 \\
13.5 \\
14.1\end{array}$ & $\begin{array}{l}12.0 \\
13.5 \\
20.0\end{array}$ & $\begin{array}{l}\text { led } \\
\text { lead } \\
304 \text { d }\end{array}$ & $\begin{array}{l}\text { lead } \\
\text { lead } \\
\text { isc }\end{array}$ & $\begin{array}{l}0.6 \\
0.6 \\
0.6\end{array}$ & $\begin{array}{l}\text { mone } \\
\text { none } \\
\text { and-b }\end{array}$ \\
\hline ⿷.13 & $\begin{array}{l}\text { tab } \\
\text { cab }\end{array}$ & $\begin{array}{l}12.0 \\
12.0 \\
13.5\end{array}$ & $\begin{array}{l}12.0 \\
12.0 \\
14.0\end{array}$ & $\begin{array}{l}12.0 \\
12.0 \\
14.0\end{array}$ & $\begin{array}{l}12.0 \\
13.5 \\
1.3\end{array}$ & $\begin{array}{l}12.0 \\
13.5 \\
20.0\end{array}$ & $\begin{array}{l}\text { led } \\
\text { lead } \\
304858\end{array}$ & $\begin{array}{l}\text { lead } \\
\text { lead } \\
\text { zirc }\end{array}$ & $\begin{array}{l}.0 \\
0.0 \\
0.0\end{array}$ & $\begin{array}{l}\text { none } \\
\text { mone } \\
\text { and-b }\end{array}$ \\
\hline E. 15 & $\begin{array}{l}1 a b \\
\operatorname{lab} \\
\operatorname{can}\end{array}$ & $\begin{array}{l}12.0 \\
12.0 \\
13.5\end{array}$ & $\begin{array}{l}12.0 \\
12.0 \\
14.0\end{array}$ & $\begin{array}{l}12.0 \\
12.0 \\
14.0\end{array}$ & $\begin{array}{l}12.0 \\
13.5 \\
14.0\end{array}$ & $\begin{array}{l}12.0 \\
13.5 \\
20.0\end{array}$ & $\begin{array}{l}\text { lead } \\
\text { lead } \\
\text { 364: }\end{array}$ & $\begin{array}{l}\text { lead } \\
\text { lead } \\
304: 5\end{array}$ & $\begin{array}{l}0.0 \\
0.0 \\
0.6\end{array}$ & $\begin{array}{l}\text { mone } \\
\text { mone } \\
\text { 1 1 1 }\end{array}$ \\
\hline 2.16 & $\begin{array}{l}\operatorname{ab} \\
\operatorname{cab} \\
\tan \end{array}$ & $\begin{array}{l}12.0 \\
12.0 \\
13.5\end{array}$ & $\begin{array}{l}12.0 \\
12.0 \\
1.0\end{array}$ & $\begin{array}{l}12.0 \\
12.0 \\
14.0\end{array}$ & $\begin{array}{l}12.0 \\
13.5 \\
14.0\end{array}$ & $\begin{array}{l}12.0 \\
13.5 \\
40.5\end{array}$ & $\begin{array}{l}\text { led } \\
\text { lead } \\
364 \text { a d }\end{array}$ & $\begin{array}{l}\text { lead } \\
1 \text { ead } \\
304 \text {. }\end{array}$ & $\begin{array}{l}.9 \\
0.0 \\
.6\end{array}$ & $\begin{array}{l}\text { 2010 } \\
\text { nore } \\
\text { c1120 }\end{array}$ \\
\hline 㹂. 17 & $\begin{array}{l}-1 a b \\
\text { cab } \\
\text { cab }\end{array}$ & $\begin{array}{l}12.0 \\
12.0 \\
13.5\end{array}$ & $\begin{array}{l}12.0 \\
12.0 \\
14.0\end{array}$ & $\begin{array}{l}12.0 \\
12.0 \\
14.0\end{array}$ & $\begin{array}{l}12.0 \\
13.5 \\
14.0\end{array}$ & $\begin{array}{l}12.0 \\
13.5 \\
20.0\end{array}$ & $\begin{array}{l}\text { lead } \\
\text { lead } \\
30458\end{array}$ & $\begin{array}{l}\text { lead } \\
\text { lead } \\
3045 \text { i }\end{array}$ & $\begin{array}{l}0.9 \\
0.0 \\
0.0\end{array}$ & $\begin{array}{l}\text { none } \\
\text { mone } \\
\text { ber t }\end{array}$ \\
\hline E. 18 & $\begin{array}{l}a b b \\
c a b \\
c a b\end{array}$ & $\begin{array}{l}12.0 \\
12.0 \\
13.5\end{array}$ & $\begin{array}{l}12.0 \\
12.0 \\
14.0\end{array}$ & $\begin{array}{l}12.0 \\
12.0 \\
14.0\end{array}$ & $\begin{array}{l}12.0 \\
13.5 \\
13.5\end{array}$ & $\begin{array}{l}12.0 \\
13.5 \\
20.0\end{array}$ & $\begin{array}{l}\text { lead } \\
\text { lead } \\
\text { eopper }\end{array}$ & $\begin{array}{l}\text { lead } \\
\text { lead } \\
\text { copor }\end{array}$ & $\begin{array}{l}0.0 \\
0.0 \\
0.0\end{array}$ & $\begin{array}{l}\text { gone } \\
\text { gone } \\
\text { ad-b }\end{array}$ \\
\hline 管。19 & $\begin{array}{l}\text { tab } \\
\text { c tab } \\
\text { can }\end{array}$ & $\begin{array}{l}12.0 \\
12.0 \\
13.5\end{array}$ & $\begin{array}{l}12.0 \\
12.0 \\
1.90\end{array}$ & $\begin{array}{l}12.0 \\
12.0 \\
14.0\end{array}$ & $\begin{array}{l}12.0 \\
13.5 \\
14.0\end{array}$ & $\begin{array}{l}12.0 \\
13.5 \\
20.0\end{array}$ & $\begin{array}{l}\text { lead } \\
\text { lead }\end{array}$ & $\begin{array}{l}\text { lead } \\
\text { lead } \\
\text { lead }\end{array}$ & $\begin{array}{l}0.0 \\
0.0 \\
0.0\end{array}$ & $\begin{array}{l}\text { mone } \\
\text { none } \\
\text { ad-b }\end{array}$ \\
\hline E.20 & $\begin{array}{l}\text { 1ab } \\
\text { c } 12 b \\
a b\end{array}$ & $\begin{array}{l}12.0 \\
12.0 \\
13.5\end{array}$ & $\begin{array}{l}12.0 \\
12.0 \\
14.0\end{array}$ & $\begin{array}{l}12.0 \\
12.0 \\
14.0\end{array}$ & $\begin{array}{l}12.0 \\
13.5 \\
14.0\end{array}$ & $\begin{array}{l}12.0 \\
13.5 \\
20.0\end{array}$ & $\begin{array}{l}\text { lead } \\
\text { lead } \\
\text { lyon }\end{array}$ & $\begin{array}{l}\text { lead } \\
\text { lead } \\
\text { jod }\end{array}$ & $\begin{array}{l}0.0 \\
0.0 \\
0.0\end{array}$ & $\begin{array}{l}\text { none } \\
\text { none } \\
\text { ad-b }\end{array}$ \\
\hline E.21 & $\begin{array}{l}\text { tab } \\
\text { eab } \\
\text { can }\end{array}$ & $\begin{array}{l}12.0 \\
12.0 \\
13.5\end{array}$ & $\begin{array}{l}12.0 \\
12.0 \\
14.0\end{array}$ & $\begin{array}{l}12.0 \\
12.0 \\
1.0\end{array}$ & $\begin{array}{l}12.0 \\
13.5 \\
14.0\end{array}$ & $\begin{array}{l}12.0 \\
13.5 \\
40.0\end{array}$ & $\begin{array}{l}\text { led } \\
\text { lead } \\
304 \text { is }\end{array}$ & $\begin{array}{l}\text { lead } \\
\text { lead } \\
3045 \text { is }\end{array}$ & $\begin{array}{l}0.0 \\
0.0 \\
0.0\end{array}$ & $\begin{array}{l}\text { none } \\
\text { none } \\
\text { nd-b }\end{array}$ \\
\hline
\end{tabular}


Table 4-2. Summary of Concepts Studied in FY'80 Follow-on Work Using Previous Version of "BARIER" Code. (Stula, 1980a). (Continued)

\begin{tabular}{|c|c|c|c|c|c|c|c|c|c|c|}
\hline Concer & 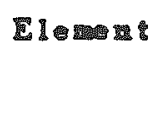 & $\begin{array}{c}11210 \\
11\end{array}$ & $\frac{1}{01}$ & $\begin{array}{c}\text { Oed } \\
\text { ID }\end{array}$ & $\begin{array}{l}10 \\
01 \\
01\end{array}$ & $\begin{array}{c}11108 \\
00\end{array}$ & 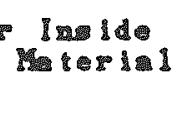 & 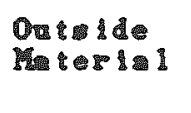 & 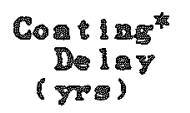 & F11 1 \\
\hline E.22 & $\begin{array}{l}12 b \\
18 b\end{array}$ & $\begin{array}{l}12.0 \\
12.0 \\
13.5\end{array}$ & $\begin{array}{l}12.0 \\
12.0 \\
14.0\end{array}$ & $\begin{array}{l}12.0 \\
12.0 \\
1.0\end{array}$ & $\begin{array}{l}12.0 \\
13.5 \\
1.2 .2\end{array}$ & $\begin{array}{l}12.0 \\
13.5 \\
40.0\end{array}$ & $\begin{array}{l}\text { lead } \\
\text { lead } \\
304 \text { is }\end{array}$ & $\begin{array}{l}\text { lead } \\
\text { lead } \\
\text { zixc }\end{array}$ & $\begin{array}{l}0.0 \\
0.0 \\
0.0\end{array}$ & $\begin{array}{l}\text { none } \\
\text { none } \\
\text { and-b }\end{array}$ \\
\hline E.23 & $\begin{array}{l}a b b \\
c a b\end{array}$ & $\begin{array}{l}12.0 \\
12.0 \\
13.0\end{array}$ & $\begin{array}{l}12.0 \\
12.0 \\
20.5\end{array}$ & $\begin{array}{l}12.0 \\
12.0 \\
20.9\end{array}$ & $\begin{array}{l}12.0 \\
13.5 \\
20.5\end{array}$ & $\begin{array}{l}12.0 \\
13.5 \\
40.0\end{array}$ & $\begin{array}{l}\text { lead } \\
\text { lead } \\
304 \text { is }\end{array}$ & $\begin{array}{l}\text { lead } \\
\text { lead } \\
3045 \text { ? }\end{array}$ & $\begin{array}{l}0.9 \\
0.0 \\
0.0\end{array}$ & $\begin{array}{l}\text { none } \\
\text { none } \\
\text { and-b }\end{array}$ \\
\hline 1.24 & $\begin{array}{l}\text { tab } \\
\text { tab } \\
c a b\end{array}$ & $\begin{array}{l}12.0 \\
12.0 \\
13.5\end{array}$ & $\begin{array}{l}12.0 \\
12.0 \\
20.5\end{array}$ & $\begin{array}{l}12.0 \\
12.0 \\
20.5\end{array}$ & $\begin{array}{l}12.0 \\
13.5 \\
20.7\end{array}$ & $\begin{array}{l}12.0 \\
13.5 \\
40.0\end{array}$ & $\begin{array}{l}\text { lead } \\
\text { lead } \\
3045 \text { i }\end{array}$ & $\begin{array}{l}\text { lead } \\
\text { lead } \\
\text { zise }\end{array}$ & $\begin{array}{l}0.0 \\
0.0 \\
0.0\end{array}$ & $\begin{array}{l}\text { none } \\
\text { none } \\
\text { and-b }\end{array}$ \\
\hline A. $15^{1}$ & 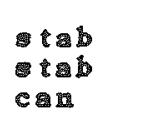 & $\begin{array}{l}12.0 \\
12.0 \\
13.5\end{array}$ & $\begin{array}{l}12.0 \\
12.0 \\
14.0\end{array}$ & $\begin{array}{l}12.0 \\
12.0 \\
1.00\end{array}$ & $\begin{array}{l}12.0 \\
13.5 \\
14.0\end{array}$ & $\begin{array}{l}12.0 \\
13.5 \\
40.0\end{array}$ & $\begin{array}{l}\text { lead } \\
\text { lead } \\
3045 \text { t }\end{array}$ & $\begin{array}{l}\text { lead } \\
\text { lead } \\
304 \text { i }\end{array}$ & $\begin{array}{l}0.0 \\
0.0 \\
0.0\end{array}$ & $\begin{array}{l}\text { none } \\
\text { none } \\
\text { mad-b }\end{array}$ \\
\hline 11.15 & $\begin{array}{l}\text { : } a b \\
\text { tab } \\
\text { can } \\
\text { leeve }\end{array}$ & $\begin{array}{l}12.0 \\
12.0 \\
13.5 \\
14.5\end{array}$ & $\begin{array}{l}12.0 \\
12.0 \\
14.0 \\
22.5\end{array}$ & $\begin{array}{l}12.0 \\
12.0 \\
14.0 \\
22.5\end{array}$ & $\begin{array}{l}12.0 \\
13.3 \\
13.0 \\
22.7\end{array}$ & $\begin{array}{l}12.0 \\
13.5 \\
10.5 \\
98.0\end{array}$ & $\begin{array}{l}\text { lead } \\
\text { led } \\
\text { tree } \\
\text { lyon }\end{array}$ & $\begin{array}{l}\text { lead } \\
\text { lead } \\
\text { tee } \\
\text { zire }\end{array}$ & $\begin{array}{l}0.0 \\
0.0 \\
0.0 \\
0.0\end{array}$ & $\begin{array}{l}\text { none } \\
\text { none } \\
\text { sand-b } \\
\text { sand-b }\end{array}$ \\
\hline C1.15 & $\begin{array}{l}\text { tab } \\
\text { cab } \\
\text { can pack } \\
\text { leeve }\end{array}$ & $\begin{array}{l}12.0 \\
12.0 \\
13.5 \\
14.5 \\
15.5\end{array}$ & $\begin{array}{l}12.0 \\
12.0 \\
14.0 \\
15.0 \\
22.5\end{array}$ & $\begin{array}{l}12.0 \\
12.0 \\
14.0 \\
15.0 \\
22.5\end{array}$ & $\begin{array}{l}12.0 \\
13.5 \\
14.0 \\
15.0 \\
22.5\end{array}$ & $\begin{array}{l}12.0 \\
13.5 \\
14.5 \\
15.5 \\
45.0\end{array}$ & 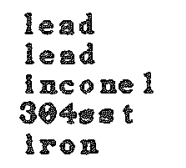 & 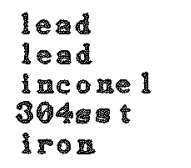 & $\begin{array}{l}0.0 \\
0.0 \\
0.0 \\
0.0 \\
0.0\end{array}$ & $\begin{array}{l}\text { none } \\
\text { none } \\
\text { and-b } \\
\text { and-b } \\
\text { and-b }\end{array}$ \\
\hline D. 35 & $\begin{array}{l}t a b \\
t a b \\
c a n \\
1 e c v e\end{array}$ & $\begin{array}{l}12.0 \\
12.0 \\
13.5 \\
14.5\end{array}$ & $\begin{array}{l}12.0 \\
12.0 \\
1.4 .0 \\
21.0\end{array}$ & $\begin{array}{l}12.0 \\
12.0 \\
14.0 \\
21.3\end{array}$ & $\begin{array}{l}12.0 \\
13.5 \\
14.0 \\
21.5\end{array}$ & $\begin{array}{l}12.0 \\
13.5 \\
14.5 \\
48.0\end{array}$ & $\begin{array}{l}\text { lead } \\
\text { lead } \\
\text { teel } \\
\text { inom }\end{array}$ & $\begin{array}{l}\text { lead } \\
\text { lead } \\
\text { tee } 1 \\
\text { lrop }\end{array}$ & $\begin{array}{r}0.0 \\
0.0 \\
100.0\end{array}$ & 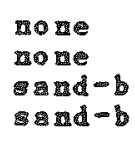 \\
\hline 01.25 & $\begin{array}{l}\text { tab } \\
\text { tab } \\
\text { can } \\
\text { leeve }\end{array}$ & $\begin{array}{l}12.0 \\
12.0 \\
13.5 \\
14.5\end{array}$ & $\begin{array}{l}12.0 \\
12.0 \\
14.0 \\
22.5\end{array}$ & $\begin{array}{l}12.0 \\
12.0 \\
14.0 \\
22.5\end{array}$ & $\begin{array}{l}12.0 \\
13.5 \\
13.0 \\
22.5\end{array}$ & $\begin{array}{l}12.0 \\
13.5 \\
14.5 \\
48.0\end{array}$ & $\begin{array}{l}\text { led } \\
\text { lead } \\
30451 \\
1 \text { yox }\end{array}$ & $\begin{array}{l}\text { lead } \\
\text { lead } \\
304 \text { t } \\
\text { lrod }\end{array}$ & $\begin{array}{r}0.0 \\
0.0 \\
0.0 \\
100.0\end{array}$ & $\begin{array}{l}\text { none } \\
\text { nowd } \\
3 \text { and-b } \\
\text { sad-b }\end{array}$ \\
\hline \multicolumn{11}{|c|}{$\begin{array}{l}\text { Coating delay represents an assumed length } \\
\text { corrosion of the coated surface would begin }\end{array}$} \\
\hline
\end{tabular}


Table 4-2. Summary of Concepts Studied in FY' 80 Follow-on Work Using Previous Version of "BARIER" Code. (Stula, 1980a). (Continued).

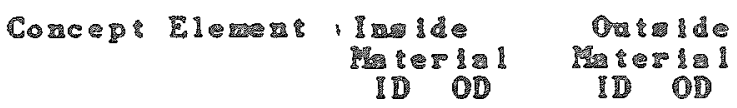

D2.15 2. $a 8 b$ $c \mathrm{ar}$ - pace leev
12.012 .0

12.012 .0 13.511 .5 .0

14.515 .0

15.53 .5
12. 12.0

12.013 .5

14.010

15.95

23.52 .5

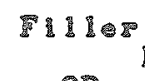
Q1)

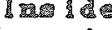

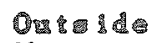

Cos

(7Fe)

12. 1ead

13.5 lea

4.

5.5 laconel

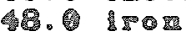

1.ed

lead

Imece

aconel

ำำ

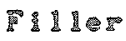

- 20 re

- 0 none

6. 군

0. 0 - a

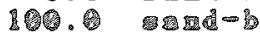


Table 4-3. Best Concepts in FY'79 Performance Study (Stula, 1980a).

\begin{tabular}{|c|c|c|c|}
\hline \multirow[b]{2}{*}{ Concept } & \multicolumn{3}{|c|}{ Release Begin Time (Yrs) - 0xic Conditions } \\
\hline & Salt & Shale & Basalt-Granite \\
\hline A.1, A.5 & 31 & 46 & \\
\hline A. 10 & & & 24,700 \\
\hline B. 8 & & & 24,700 \\
\hline B1.7 & & & 1100 \\
\hline B1.11 & 1920 & 1930 & \\
\hline C.7 & & & 49,400 \\
\hline $\mathrm{Cl} .1, \mathrm{Cl} .3$ & 27 & 41 & \\
\hline $\mathrm{Cl} .6$ & & & 11,900 \\
\hline D. 1 & & 54 & \\
\hline 0.3 & 130 & & \\
\hline D. 5 & & & 24,800 \\
\hline 01.2 & 140 & 150 & \\
\hline D1. 5 & & & 1700 \\
\hline 02.1 & 140 & 150 & \\
\hline 02.8 & & & 1900 \\
\hline E. 3 & 2600 & 3500 & \\
\hline
\end{tabular}



Table 4-4. Summary of Concepts Studied With New Version of "BARIER" Code. (Unless Otherwise Noted, Dimensions in Inches).

\begin{tabular}{|c|c|c|c|c|c|c|c|c|c|c|}
\hline CONCEPT & ELEMTENT & $\begin{array}{l}\text { INS } \\
\text { RATTE } \\
\text { ID }\end{array}$ & $\begin{array}{l}\text { IDE } \\
\text { RIAL } \\
\text { OD }\end{array}$ & $\begin{array}{l}\text { OUTS IDE } \\
\text { MATERIAAL } \\
\text { OD }\end{array}$ & $\begin{array}{c}\text { PILLER } \\
\text { OD }\end{array}$ & $\begin{array}{r}\text { GAP } \\
\text { OD }\end{array}$ & $\begin{array}{l}\text { INS IDE. } \\
\text { MATERIAL }\end{array}$ & $\begin{array}{l}\text { OUTSIDE } \\
\text { PATER IAL }\end{array}$ & $\begin{array}{c}\text { COATING } \\
\text { DELAY } \\
\text { (YRS) }\end{array}$ & FILLER \\
\hline A. 1 & $\begin{array}{l}\text { STAB } \\
\text { CAIt }\end{array}$ & $\begin{array}{l}12.0 \\
13.5\end{array}$ & $\begin{array}{l}13.8 \\
14.0\end{array}$ & $\begin{array}{l}13.5 \\
19.0\end{array}$ & $\begin{array}{l}13.5 \\
40.0\end{array}$ & $\begin{array}{l}13.5 \\
40.0\end{array}$ & $\begin{array}{l}30455 T \\
3045 S T\end{array}$ & $\begin{array}{l}3045 S T \\
3045 S T\end{array}$ & 0.0 & $\begin{array}{l}\text { MONE } \\
\text { SAND-B }\end{array}$ \\
\hline A. 5 & $\begin{array}{l}\text { STAB } \\
\text { CAN }\end{array}$ & $\begin{array}{l}12.0 \\
19.5\end{array}$ & $\begin{array}{l}13.5 \\
15.5\end{array}$ & $\begin{array}{l}13.5 \\
15.5\end{array}$ & $\begin{array}{l}13.5 \\
20.0\end{array}$ & $\begin{array}{l}13.5 \\
20.0\end{array}$ & $\begin{array}{l}3045 S T \\
3045 S T\end{array}$ & $\begin{array}{l}\text { 304SST } \\
\text { 304SST }\end{array}$ & 0.0 & $\begin{array}{l}\text { NONE } \\
\text { SAMD }-B\end{array}$ \\
\hline A. 10 & $\begin{array}{l}\text { STAB } \\
\text { CAN }\end{array}$ & $\begin{array}{l}12.0 \\
13.5\end{array}$ & $\begin{array}{l}13.5 \\
15.5\end{array}$ & $\begin{array}{l}13.5 \\
15.3\end{array}$ & $\begin{array}{l}13.5 \\
20.0\end{array}$ & $\begin{array}{l}13.5 \\
20.0\end{array}$ & $\begin{array}{l}\text { ZIRC } \\
\text { ZIRC }\end{array}$ & $\begin{array}{l}\text { ZIRC } \\
\text { ZIRC }\end{array}$ & 0.0 & $\begin{array}{l}\text { FONE } \\
\text { SAND-B }\end{array}$ \\
\hline B. 8 & $\begin{array}{l}\text { STAB } \\
\text { CAN } \\
0 \text { PACK }\end{array}$ & $\begin{array}{l}12.0 \\
13.5 \\
14.5\end{array}$ & $\begin{array}{l}13.5 \\
14.0 \\
16.5\end{array}$ & $\begin{array}{l}13.5 \\
14.0 \\
16.5\end{array}$ & $\begin{array}{l}13.5 \\
14.5 \\
20.0\end{array}$ & $\begin{array}{l}13.5 \\
14.5 \\
20.0\end{array}$ & $\begin{array}{l}\text { STEEL } \\
\text { STEEL } \\
\text { ZIRC }\end{array}$ & $\begin{array}{l}\text { STEEL } \\
\text { STEEL } \\
\text { ZIRC }\end{array}$ & $\begin{array}{l}0.0 \\
0.6 \\
0.6\end{array}$ & $\begin{array}{l}\text { NONE } \\
\text { SAND-B } \\
\text { SAND-B }\end{array}$ \\
\hline$B 1.7$ & $\begin{array}{l}\text { STAB } \\
\text { CAN } \\
\text { SLEVER }\end{array}$ & $\begin{array}{l}12.0 \\
13.5 \\
14.5\end{array}$ & $\begin{array}{l}13.5 \\
14.0 \\
15.0\end{array}$ & $\begin{array}{l}13.5 \\
14.0 \\
23.0\end{array}$ & $\begin{array}{l}13.5 \\
14.5 \\
30.0\end{array}$ & $\begin{array}{l}13.5 \\
14.5 \\
30.0\end{array}$ & $\begin{array}{l}\text { STEEL } \\
\text { STEEL } \\
\text { STEEL }\end{array}$ & $\begin{array}{l}\text { STEEL } \\
\text { STEEL } \\
\text { LEAD }\end{array}$ & $\begin{array}{l}0.0 \\
0.0 \\
0.0\end{array}$ & $\begin{array}{l}\text { RONE } \\
\text { SAND-B } \\
\text { CLINO }\end{array}$ \\
\hline B1. 11 & $\begin{array}{l}\text { STAB } \\
\text { CAN } \\
\text { SLEEVE }\end{array}$ & $\begin{array}{l}12.0 \\
13.5 \\
14.5\end{array}$ & $\begin{array}{l}13.5 \\
14.0 \\
22.5\end{array}$ & $\begin{array}{l}13.5 \\
14.0 \\
22.7\end{array}$ & $\begin{array}{l}13.5 \\
14.5 \\
48.0\end{array}$ & $\begin{array}{l}13.5 \\
14.7 \\
88.9\end{array}$ & $\begin{array}{l}\text { STEEL } \\
\text { STEEL } \\
\text { IRON }\end{array}$ & $\begin{array}{l}\text { \$TEEL } \\
\text { STEEL } \\
\text { ZIMC }\end{array}$ & $\begin{array}{l}9.0 \\
9.9 \\
0.0\end{array}$ & $\begin{array}{l}\text { NONE } \\
\text { SAND-B } \\
\text { SAND-B }\end{array}$ \\
\hline B1. $1 \mathrm{~N}$ & $\begin{array}{l}\text { STAB } \\
\text { CAN } \\
\text { SLECVE }\end{array}$ & $\begin{array}{l}13.0 \\
13.5 \\
14.5\end{array}$ & $\begin{array}{l}13.5 \\
14.0 \\
15.0\end{array}$ & $\begin{array}{l}13.5 \\
14.0 \\
15.0\end{array}$ & $\begin{array}{l}13.5 \\
14.5 \\
411.0\end{array}$ & $\begin{array}{l}13.5 \\
14.5 \\
48.0\end{array}$ & $\begin{array}{l}\text { STEEL } \\
\text { STERL } \\
\text { IRON }\end{array}$ & $\begin{array}{l}\text { STEEL } \\
\text { STEEL } \\
\text { IRON }\end{array}$ & $\begin{array}{l}0.0 \\
0.0 \\
0.0\end{array}$ & $\begin{array}{l}\text { NONE } \\
\text { SAND-B } \\
\text { SAND-B }\end{array}$ \\
\hline B1. $2 \mathrm{~N}$ & $\begin{array}{l}\text { STAB } \\
\text { CAR } \\
\text { SLEEVE }\end{array}$ & $\begin{array}{l}12.0 \\
13.5 \\
14.5\end{array}$ & $\begin{array}{l}13.5 \\
14.0 \\
21.5\end{array}$ & $\begin{array}{l}13.5 \\
14.0 \\
21.5\end{array}$ & $\begin{array}{l}13.5 \\
14.5 \\
48.0\end{array}$ & $\begin{array}{l}13.5 \\
14.5 \\
48.0\end{array}$ & $\begin{array}{l}\text { STEEL } \\
\text { STEEL } \\
\text { IRUN }\end{array}$ & $\begin{array}{l}\text { STEEL } \\
\text { STEEL } \\
\text { IRON }\end{array}$ & $\begin{array}{l}0.0 \\
0.0 \\
0.0\end{array}$ & $\begin{array}{l}\text { NONE } \\
\text { SAND-B } \\
\text { SAND-B }\end{array}$ \\
\hline $3 \pi$ & $\begin{array}{l}\text { STAB } \\
\text { CAN }\end{array}$ & $\begin{array}{l}12.0 \\
13.5\end{array}$ & $\begin{array}{l}13.5 \\
14.0\end{array}$ & $\begin{array}{l}13.5 \\
14.0\end{array}$ & $\begin{array}{l}19.5 \\
14.5\end{array}$ & $\begin{array}{l}13.5 \\
14.5\end{array}$ & $\begin{array}{l}\text { STEEL } \\
\text { STEEL }\end{array}$ & $\begin{array}{l}\text { STEEL } \\
\text { STEEL }\end{array}$ & 9.0 & $\begin{array}{l}\text { NONE } \\
\text { SAND-B }\end{array}$ \\
\hline
\end{tabular}


Table 4-4. (Continued)

\begin{tabular}{|c|c|c|c|c|c|c|c|c|c|c|}
\hline COHCEPT & ELEMET & 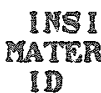 & 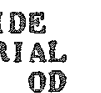 & $\begin{array}{l}\text { OIIS IDE } \\
\text { METREIAL } \\
\text { OD }\end{array}$ & $\begin{array}{c}\text { PLLER } \\
\text { O1 }\end{array}$ & $\begin{array}{l}\text { GAP } \\
\text { OD }\end{array}$ & $\begin{array}{r}\text { INS IDE: } \\
\text { MATRERIAL }\end{array}$ & $\begin{array}{l}\text { OUTS IDE: } \\
\text { MATERIAL: }\end{array}$ & $\begin{array}{l}\text { COATIRG } \\
\text { DELAY } \\
\text { (YRS) }\end{array}$ & 1 LLER \\
\hline D1.31 & SLFEVE & 14. & 26.5 & 26.5 & 4 & ㄱ. & MON & IRON & 0.0 & $S A R D-B$ \\
\hline 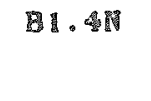 & $\begin{array}{l}\text { STAB } \\
\text { CAN } \\
\text { SLEEVE }\end{array}$ & $\begin{array}{l}12.0 \\
13.5 \\
18.5\end{array}$ & $\begin{array}{l}13.5 \\
1.60 \\
21.50\end{array}$ & $\begin{array}{l}10.5 \\
14.0 \\
31.6\end{array}$ & $\begin{array}{l}13.5 \\
14.5 \\
618.5\end{array}$ & $\begin{array}{l}13.5 \\
14.58 \\
40.0\end{array}$ & $\begin{array}{l}\text { STEEL } \\
\text { STIEEL } \\
\text { TRON }\end{array}$ & $\begin{array}{l}\text { STEEL } \\
\text { STERE } \\
\text { ZIRC }\end{array}$ & $\begin{array}{r}9.0 \\
6.0 \\
6.0\end{array}$ & $\begin{array}{l}\text { PONIR } \\
\text { SAND-B } \\
\text { SAND-D }\end{array}$ \\
\hline 01.518 & $\begin{array}{l}\text { STAB } \\
\text { CAN } \\
\text { SLERVE }\end{array}$ & $\begin{array}{l}12.0 \\
13.5 \\
14.5\end{array}$ & $\begin{array}{l}13.5 \\
14.0 \\
31.5\end{array}$ & $\begin{array}{l}19.5 \\
1 \% .0 \\
21.8\end{array}$ & $\begin{array}{l}13.5 \\
13.5 \\
43.5\end{array}$ & $\begin{array}{l}13.5 \\
13.5 \\
46.5\end{array}$ & $\begin{array}{l}\text { STREL } \\
\text { STERL } \\
\text { IRON }\end{array}$ & $\begin{array}{l}\text { STEEL } \\
\text { STEEL } \\
\text { ZIRC }\end{array}$ & $\begin{array}{l}0.0 \\
0.0 \\
0.0\end{array}$ & $\begin{array}{l}\text { NOHE } \\
\text { SAND-B } \\
\text { SAND-B }\end{array}$ \\
\hline 81.6醒 & $\begin{array}{l}\text { STAB } \\
\text { CAIR } \\
\text { 3LEREE }\end{array}$ & $\begin{array}{l}12.9 \\
13.5 \\
14.5\end{array}$ & $\begin{array}{l}13.5 \\
14.0 \\
31.5\end{array}$ & $\begin{array}{l}13.5 \\
14.0 \\
23.0\end{array}$ & 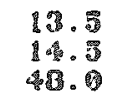 & $\begin{array}{l}13.5 \\
13.5 \\
48.9\end{array}$ & $\begin{array}{l}\text { STEEL } \\
\text { STEEL } \\
\text { INON }\end{array}$ & $\begin{array}{l}\text { STEEL } \\
\text { STEEL } \\
\text { ZIRC }\end{array}$ & $\begin{array}{l}0.0 \\
0.0 \\
0.0\end{array}$ & $\begin{array}{l}\text { NONE } \\
\text { SAND- } \\
\text { SAND-B }\end{array}$ \\
\hline B1. & $\begin{array}{l}\text { STAO } \\
\text { CAN } \\
\text { SELEVER }\end{array}$ & $\begin{array}{l}12.0 \\
13.5 \\
13.5\end{array}$ & $\begin{array}{l}13.5 \\
14.5 \\
15.5\end{array}$ & $\begin{array}{l}13.5 \\
14.5 \\
15.3\end{array}$ & $\begin{array}{l}13.5 \\
14.5 \\
48.5\end{array}$ & $\begin{array}{l}13.5 \\
13.5 \\
48.5\end{array}$ & $\begin{array}{l}\text { STEEL } \\
\text { \$TEEL } \\
\text { I NON }\end{array}$ & $\begin{array}{l}\text { STEEL } \\
\text { STEEL } \\
\text { ZIRC }\end{array}$ & $\begin{array}{l}0.0 \\
0.0 \\
0.0\end{array}$ & $\begin{array}{l}\text { PONE } \\
\text { SAND- } \\
\text { SAND-B }\end{array}$ \\
\hline B1. 8 IN & $\begin{array}{l}\text { STAB } \\
\text { CALE } \\
\text { SELEVI }\end{array}$ & $\begin{array}{l}12.0 \\
12.5 \\
14.5\end{array}$ & $\begin{array}{l}13.5 \\
14.5 \\
26.5\end{array}$ & $\begin{array}{l}13.5 \\
14.0 \\
26.0\end{array}$ & $\begin{array}{l}10.5 \\
104.5 \\
68.5\end{array}$ & $\begin{array}{l}13.5 \\
13.5 \\
48.6\end{array}$ & $\begin{array}{l}\text { STEEL } \\
\text { STELL } \\
\text { IROR }\end{array}$ & $\begin{array}{l}\text { STEEL } \\
\text { STEEL } \\
\text { ZIRC }\end{array}$ & $\begin{array}{l}9.1 \\
0.0 \\
0.0\end{array}$ & $\begin{array}{l}\text { NONE } \\
\text { SAND-D } \\
\text { SAND-D }\end{array}$ \\
\hline 21.98 & $\begin{array}{l}\text { GTAB } \\
\text { CAN } \\
\text { SLEVER }\end{array}$ & $\begin{array}{l}12.0 \\
13.5 \\
1.5 .5\end{array}$ & $\begin{array}{l}12.5 \\
14.0 \\
31.5\end{array}$ & $\begin{array}{l}13.5 \\
13.0 \\
21.6\end{array}$ & $\begin{array}{l}13.5 \\
13.5 \\
40.0\end{array}$ & $\begin{array}{l}13.5 \\
14.5 \\
48 .\end{array}$ & $\begin{array}{l}\text { STLEL } \\
\text { STRELL } \\
\text { 304SST }\end{array}$ & $\begin{array}{l}\text { TLEL } \\
\text { ITEEL } \\
\text { ZIRC }\end{array}$ & 10.0 & $\begin{array}{l}\text { NONE } \\
\text { SAND-B } \\
\text { SAND-B }\end{array}$ \\
\hline 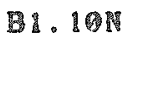 & $\begin{array}{l}\text { STAB } \\
\text { CAND } \\
\text { SLEEVE }\end{array}$ & $\begin{array}{l}12.5 \\
13.9 \\
14.3\end{array}$ & $\begin{array}{l}13.5 \\
14.0 \\
21.5\end{array}$ & $\begin{array}{l}13.5 \\
14.0 \\
22.0\end{array}$ & $\begin{array}{l}13.5 \\
13.5 \\
48.5\end{array}$ & $\begin{array}{l}13.5 \\
13.5 \\
48.9\end{array}$ & $\begin{array}{l}\text { STELL } \\
\text { STERL } \\
3545 S T\end{array}$ & $\begin{array}{l}\text { STERL } \\
\text { STERL } \\
\text { ZIRC }\end{array}$ & $\begin{array}{l}9.9 \\
7.0 \\
6.9\end{array}$ & $\begin{array}{l}\text { RORE } \\
\text { SAND-B } \\
\text { SAND-B }\end{array}$ \\
\hline 81.111 & $\begin{array}{l}\text { STAR } \\
\text { CAR } \\
\text { SALEVER }\end{array}$ & $\begin{array}{l}12.0 \\
13.5 \\
14.5\end{array}$ & $\begin{array}{l}13.5 \\
14.0 \\
15.50\end{array}$ & $\begin{array}{l}13.5 \\
14.5 \\
15.0\end{array}$ & $\begin{array}{l}13.5 \\
13.5 \\
40.0\end{array}$ & $\begin{array}{l}13.5 \\
13.5 \\
40.0\end{array}$ & $\begin{array}{l}\text { 3TEEL } \\
\text { STERE } \\
\text { COPPRT }\end{array}$ & $\begin{array}{l}\text { STEEL } \\
\text { STEEL } \\
\text { COPPER }\end{array}$ & $\begin{array}{l}9.9 \\
9.9 \\
0.0\end{array}$ & $\begin{array}{l}\text { MONE } \\
\text { SAND-B } \\
\text { SAND-B }\end{array}$ \\
\hline
\end{tabular}


Tabie 4-4. (Continued)

\begin{tabular}{|c|c|c|c|c|c|c|c|c|c|c|}
\hline CORCEPT & ELEPRERT & $\begin{array}{c}\text { IITS } \\
\text { MATRR } \\
\text { II }\end{array}$ & $\begin{array}{l}\text { IDE } \\
\text { DIAL } \\
\text { OD }\end{array}$ & $\begin{array}{c}\text { ORTSIDE } \\
\text { WATEMIAL } \\
\text { OD }\end{array}$ & $\begin{array}{c}\text { FILLER } \\
\text { OD }\end{array}$ & $\begin{array}{r}\text { GAP } \\
\text { OD }\end{array}$ & $\begin{array}{l}\text { IRS IDE: } \\
\text { YUTLR IAL }\end{array}$ & $\begin{array}{l}\text { OVTS IDE } \\
\text { IMTCMIAL: }\end{array}$ & $\begin{array}{l}\text { COATING } \\
\text { DELAY } \\
\text { (YRS) }\end{array}$ & PILLET \\
\hline BH.12N & $\begin{array}{l}\text { STHB } \\
\text { CAIT } \\
\text { SLLEVE }\end{array}$ & $\begin{array}{l}12.0 \\
13.5 \\
14.53\end{array}$ & $\begin{array}{l}13.5 \\
13.0 \\
21.5\end{array}$ & $\begin{array}{l}13.5 \\
13.5 \\
21.9 \\
21.9\end{array}$ & $\begin{array}{l}13.50 \\
14.5 \\
48.5 \\
48.07\end{array}$ & $\begin{array}{l}13.9 \\
13.5 \\
48.0\end{array}$ & $\begin{array}{l}\text { STEEL } \\
\text { STREL } \\
\text { COPPRR. }\end{array}$ & $\begin{array}{l}\text { STELL } \\
\text { STEEL } \\
\text { COPPER }\end{array}$ & $\begin{array}{l}0.0 \\
0.0 \\
0.0\end{array}$ & $\begin{array}{l}\text { PONE } \\
\text { SAND }-\mathbb{B} \\
\text { SAND }- \text { R }\end{array}$ \\
\hline D1.13得 & $\begin{array}{l}\text { STAB } \\
\text { CAN } \\
\text { SLEEVE }\end{array}$ & $\begin{array}{l}12.0 \\
13.5 \\
12.5\end{array}$ & $\begin{array}{l}13.5 \\
18.0 \\
13.0\end{array}$ & $\begin{array}{l}13.5 \\
13.0 \\
15.0\end{array}$ & $\begin{array}{l}13.5 \\
11.5 \\
46.0\end{array}$ & $\begin{array}{l}13.5 \\
13.5 \\
148.5\end{array}$ & $\begin{array}{l}\text { STEEL } \\
\text { STEEL } \\
\text { IMCONEL }\end{array}$ & $\begin{array}{l}\text { STEEL } \\
\text { STEEL } \\
\text { INCONEL }\end{array}$ & $\begin{array}{l}0.0 \\
0.0 \\
0.0\end{array}$ & $\begin{array}{l}\text { MONE } \\
\text { SAND-B } \\
\text { SAND-B }\end{array}$ \\
\hline B1.14 & $\begin{array}{l}\text { GTAD } \\
\text { CAII } \\
\text { SLLERER }\end{array}$ & $\begin{array}{l}13.0 \\
13.5 \\
14.5\end{array}$ & $\begin{array}{l}13.5 \\
19.8 \\
21.5\end{array}$ & $\begin{array}{l}13.5 \\
13.5 \\
21.5\end{array}$ & $\begin{array}{l}13.5 \\
13.5 \\
1 \% .5 \\
38.0\end{array}$ & $\begin{array}{l}13.5 \\
19.5 \\
46.6\end{array}$ & $\begin{array}{l}\text { STEEL } \\
\text { STEEL } \\
\text { INCOREL }\end{array}$ & $\begin{array}{l}\text { STEEL } \\
\text { STERL } \\
\text { INCOPRL }\end{array}$ & $\begin{array}{l}0.0 \\
0.0 \\
0.0\end{array}$ & $\begin{array}{l}\text { PONE } \\
\text { SAPD-D } \\
\text { SARD-B }\end{array}$ \\
\hline B1.15T & $\begin{array}{l}\text { STAB } \\
\text { CAR } \\
\text { SLPEVE }\end{array}$ & $\begin{array}{l}12.0 \\
13.5 \\
14.5\end{array}$ & $\begin{array}{l}13.5 \\
14.0 \\
15.0\end{array}$ & $\begin{array}{l}13.5 \\
19.5 \\
13.0\end{array}$ & $\begin{array}{l}13.5 \\
13.5 \\
19.5\end{array}$ & $\begin{array}{l}13.5 \\
14.5 \\
48.0\end{array}$ & $\begin{array}{l}\text { STEEL } \\
\text { STLEL } \\
30465 T\end{array}$ & $\begin{array}{l}\text { STELL } \\
\text { STEEL } \\
3045 S T\end{array}$ & $\begin{array}{l}3.0 \\
0.0 \\
0.0\end{array}$ & $\begin{array}{l}\text { NONE } \\
\text { SAND-B } \\
\text { SAND-B }\end{array}$ \\
\hline 31.167 & $\begin{array}{l}\text { STAR } \\
\text { CAMN } \\
\text { SLEEVE }\end{array}$ & $\begin{array}{l}12.0 \\
12.5 \\
14.53\end{array}$ & 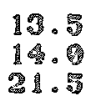 & $\begin{array}{l}13.5 \\
44.5 \\
31.5\end{array}$ & 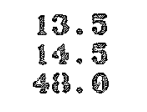 & $\begin{array}{l}13.5 \\
13.3 \\
40.5\end{array}$ & $\begin{array}{l}\text { STEEL } \\
\text { STREL } \\
30455 T\end{array}$ & $\begin{array}{l}\text { STEE } \\
\text { STEEL } \\
\text { כ2ASST }\end{array}$ & $\begin{array}{l}0.0 \\
0.0\end{array}$ & $\begin{array}{l}\text { MONE } \\
\text { SAND-B } \\
\text { SAND- }\end{array}$ \\
\hline 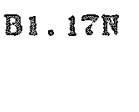 & $\begin{array}{l}\text { STAB } \\
\text { CAN } \\
\text { SLEEVE }\end{array}$ & $\begin{array}{l}12.0 \\
13.5 \\
13.5\end{array}$ & $\begin{array}{l}13.5 \\
13.0 \\
19.0\end{array}$ & $\begin{array}{l}13.5 \\
14.0 \\
15.0\end{array}$ & $\begin{array}{l}13.5 \\
14.5 \\
14.5 \\
48.5\end{array}$ & $\begin{array}{l}13.5 \\
14.5 \\
48.5\end{array}$ & $\begin{array}{l}\text { STEEL } \\
\text { STEEL } \\
\text { ZIRC }\end{array}$ & $\begin{array}{l}\text { STEEL } \\
\text { STEEL } \\
\text { ZIVE }\end{array}$ & $\begin{array}{l}0.0 \\
0.0 \\
0.0\end{array}$ & $\begin{array}{l}\text { NONE } \\
\text { SAND-D } \\
\text { SAND-B }\end{array}$ \\
\hline B1. 184 & $\begin{array}{l}\text { STAB } \\
\text { GAN } \\
\text { SLEEVE }\end{array}$ & $\begin{array}{l}12.0 \\
13.5 \\
13.5\end{array}$ & $\begin{array}{l}13.5 \\
13.5 \\
21.9 \\
21.9\end{array}$ & $\begin{array}{l}13.5 \\
14.0 \\
21.5\end{array}$ & $\begin{array}{l}13.5 \\
13.5 \\
40.3\end{array}$ & 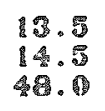 & $\begin{array}{l}\text { STEEL } \\
\text { STERL } \\
\text { EIRC }\end{array}$ & $\begin{array}{l}\text { STEEL } \\
\text { STEEL } \\
\text { ZIRG }\end{array}$ & $\begin{array}{l}9.0 \\
0.0 \\
0.0\end{array}$ & $\begin{array}{l}\text { PONE } \\
\text { SAND-B } \\
\text { SAND-B }\end{array}$ \\
\hline B1. 19: & $\begin{array}{l}\text { STAB } \\
\text { CAR } \\
\text { SLEEVE }\end{array}$ & $\begin{array}{l}12.0 \\
13.5 \\
1 \% .5\end{array}$ & $\begin{array}{l}13.9 \\
13.0 \\
19.5 \\
21.5\end{array}$ & $\begin{array}{l}13.9 \\
13.0 \\
11.6 \\
21.6\end{array}$ & $\begin{array}{l}13.5 \\
19.5 \\
48.0\end{array}$ & $\begin{array}{l}13.5 \\
13.5 \\
48.0\end{array}$ & $\begin{array}{l}\text { STEEL } \\
\text { STEEL } \\
\text { IRON }\end{array}$ & $\begin{array}{l}\text { STEEL } \\
\text { STEEL } \\
\text { ZIRC }\end{array}$ & $\begin{array}{l}0.9 \\
0.9 \\
0.9\end{array}$ & $\begin{array}{l}\text { MONR } \\
\text { SAND-B } \\
\text { SMND-D }\end{array}$ \\
\hline B1.39: & $\begin{array}{l}\text { \$TAB } \\
\text { CAIR } \\
\text { SLEVE }\end{array}$ & $\begin{array}{l}12.09 \\
13 . \\
13.9\end{array}$ & $\begin{array}{l}13.5 \\
13.0 \\
21.5\end{array}$ & $\begin{array}{l}13.5 \\
13.0 \\
21.8\end{array}$ & $\begin{array}{l}13.5 \\
13.5 \\
14.8 \\
46.63\end{array}$ & $\begin{array}{l}13.5 \\
14.5 \\
48.5\end{array}$ & $\begin{array}{l}\text { STEEL } \\
\text { STEEL } \\
\text { IRON }\end{array}$ & $\begin{array}{l}\text { STEEL } \\
\text { STEEL } \\
\text { ZINC }\end{array}$ & $\begin{array}{l}0.0 \\
0.0\end{array}$ & $\begin{array}{l}\text { MONE } \\
\text { SAND-B } \\
\text { SAND-B }\end{array}$ \\
\hline
\end{tabular}


Table 4-4. (Continued)

\begin{tabular}{|c|c|c|c|c|c|c|c|c|c|c|}
\hline CONCEPT & ELENTRT & 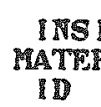 & $\begin{array}{l}\text { IDE } \\
\text { MIAL } \\
\text { OD }\end{array}$ & $\begin{array}{l}\text { OUTSIDE } \\
\text { RATERIAL } \\
\text { OD }\end{array}$ & $\begin{array}{l}\text { FLLER } \\
\text { OD }\end{array}$ & $\begin{array}{r}\text { GAP } \\
\text { OD }\end{array}$ & $\begin{array}{r}\text { INS IDEU } \\
\text { HATRR IAL }\end{array}$ & $\begin{array}{l}\text { OUTS IDE } \\
\text { AATRRIAL }\end{array}$ & $\begin{array}{l}\text { COATIRG } \\
\text { DELAY } \\
\text { (YRS) }\end{array}$ & F MLLER \\
\hline B1.211 & $\begin{array}{l}\text { STAB } \\
\text { CAN } \\
\text { SLEEVE }\end{array}$ & $\begin{array}{l}12.0 \\
13.5 \\
14.5\end{array}$ & $\begin{array}{l}12.5 \\
14.0 \\
21.3\end{array}$ & $\begin{array}{l}13.5 \\
14.0 \\
22.0\end{array}$ & $\begin{array}{l}13.5 \\
14.5 \\
48.0\end{array}$ & $\begin{array}{l}13.5 \\
14.5 \\
48.0\end{array}$ & $\begin{array}{l}\text { STERL } \\
\text { THELL } \\
\text { MONON }\end{array}$ & $\begin{array}{l}\text { 5TELL } \\
\text { TIEEL } \\
\text { ZIIC }\end{array}$ & $\begin{array}{l}0.0 \\
0.0 \\
0.0\end{array}$ & $\begin{array}{l}\text { MONE } \\
\text { SAND-B } \\
\text { SAND-B }\end{array}$ \\
\hline B1. $22 N$ & $\begin{array}{l}\text { STAB } \\
\text { CAN } \\
\text { SLEEVE }\end{array}$ & $\begin{array}{l}12.0 \\
13.5 \\
14.5\end{array}$ & $\begin{array}{l}13.5 \\
14.0 \\
15.0\end{array}$ & $\begin{array}{l}13.5 \\
14.0 \\
15.3\end{array}$ & $\begin{array}{l}13.5 \\
14.5 \\
40.0\end{array}$ & $\begin{array}{l}13.5 \\
14.5 \\
40.0\end{array}$ & $\begin{array}{l}\text { STEEL } \\
\text { STELL } \\
\text { IRON }\end{array}$ & $\begin{array}{l}\text { STEEL } \\
\text { STEELL } \\
\text { ZIRC }\end{array}$ & $\begin{array}{l}0.0 \\
0.0 \\
0.0\end{array}$ & $\begin{array}{l}\text { NONE } \\
\text { SAND-B } \\
\text { SAND-B }\end{array}$ \\
\hline B1. $23 N$ & $\begin{array}{l}\text { STAB } \\
\text { CAN } \\
\text { SLEEVE }\end{array}$ & $\begin{array}{l}12.0 \\
12.5 \\
14.5\end{array}$ & $\begin{array}{l}13.5 \\
14.0 \\
26.5\end{array}$ & $\begin{array}{l}13.5 \\
14.0 \\
26.8\end{array}$ & $\begin{array}{l}13.5 \\
14.5 \\
48.9\end{array}$ & $\begin{array}{l}13.5 \\
14.5 \\
43.0\end{array}$ & $\begin{array}{l}\text { STEEL } \\
\text { STEEL } \\
\text { IRON }\end{array}$ & $\begin{array}{l}\text { STREL } \\
\text { STEEL } \\
\text { ZIRG }\end{array}$ & $\begin{array}{l}0.0 \\
0.0\end{array}$ & $\begin{array}{l}\text { NONE } \\
\text { SAND-B } \\
\text { SAND-B }\end{array}$ \\
\hline B1.24N & $\begin{array}{l}\text { STAB } \\
\text { CAN } \\
\text { SLEEVE }\end{array}$ & $\begin{array}{l}12.5 \\
13.5 \\
14.5\end{array}$ & $\begin{array}{l}12.0 \\
14.0 \\
21.5\end{array}$ & $\begin{array}{l}12.0 \\
14.0 \\
21 .\end{array}$ & $\begin{array}{l}12.0 \\
14.7 \\
48.0\end{array}$ & $\begin{array}{l}13.5 \\
14.5 \\
48.0\end{array}$ & $\begin{array}{l}\text { HELIUT. } \\
\text { STEEL } \\
\text { InON }\end{array}$ & $\begin{array}{l}\text { HELIUM } \\
\text { STEEL } \\
\text { IRON }\end{array}$ & $\begin{array}{l}9.0 \\
0.0 \\
0.0\end{array}$ & $\begin{array}{l}\text { NONE } \\
\text { SAND-B } \\
\text { SAND-B }\end{array}$ \\
\hline B1.35N & $\begin{array}{l}\text { \$TAD } \\
\text { CAN } \\
\text { SLEVVE }\end{array}$ & $\begin{array}{l}12.0 \\
13.5 \\
14.5\end{array}$ & $\begin{array}{l}12.0 \\
11.0 \\
21.5\end{array}$ & $\begin{array}{l}12.0 \\
14.0 \\
21.5\end{array}$ & $\begin{array}{l}12.0 \\
14.5 \\
48.0\end{array}$ & $\begin{array}{l}13.5 \\
14.5 \\
48.0\end{array}$ & $\begin{array}{l}\text { AIR } \\
\text { STEEL } \\
\text { InON }\end{array}$ & $\begin{array}{l}\text { AIR } \\
\text { STEEL } \\
\text { IRON }\end{array}$ & $\begin{array}{l}0.0 \\
0.0\end{array}$ & $\begin{array}{l}\text { NONE } \\
\text { SAND-B } \\
\text { SAND-B }\end{array}$ \\
\hline B1. $26 N$ & $\begin{array}{l}\text { STAB } \\
\text { CAN } \\
\text { SLERVE }\end{array}$ & $\begin{array}{l}12.9 \\
13.5 \\
14.5\end{array}$ & $\begin{array}{l}12.0 \\
14.0 \\
21.5\end{array}$ & $\begin{array}{l}12.0 \\
11.0 \\
21.5\end{array}$ & $\begin{array}{l}12.0 \\
1 \% .0 \\
40.0\end{array}$ & $\begin{array}{l}13.5 \\
18.5 \\
48.0\end{array}$ & $\begin{array}{l}\text { HELIUM. } \\
\text { STEEL } \\
\text { IRON }\end{array}$ & $\begin{array}{l}\text { EELIUM } \\
\text { STEEL } \\
\text { IRON }\end{array}$ & $\begin{array}{l}0.0 \\
0.0 \\
0.0\end{array}$ & $\begin{array}{l}\text { RONE } \\
\text { RONE } \\
\text { SAND-B }\end{array}$ \\
\hline $\mathrm{B1} \cdot \mathrm{2}-\mathrm{N}$ & $\begin{array}{l}\text { STAB } \\
\text { GAN } \\
\text { SLEEVE }\end{array}$ & $\begin{array}{l}12.0 \\
13.5 \\
13.0\end{array}$ & $\begin{array}{l}12.0 \\
14.0 \\
22.0\end{array}$ & $\begin{array}{l}12.0 \\
14.0 \\
22.0\end{array}$ & $\begin{array}{r}12.0 \\
14.9 \\
43.5\end{array}$ & $\begin{array}{r}13.5 \\
15.9 \\
48.5\end{array}$ & $\begin{array}{l}\text { HELIUM } \\
\text { STEEL } \\
\text { IRON }\end{array}$ & $\begin{array}{l}\text { HEL IUM } \\
\text { STEEL } \\
\text { IRON }\end{array}$ & $\begin{array}{l}0.0 \\
0.0 \\
0.0\end{array}$ & $\begin{array}{l}\text { NONE } \\
\text { NONE } \\
\text { AND-B }\end{array}$ \\
\hline B1. $28 \mathrm{~N}$ & $\begin{array}{l}\text { STMB } \\
\text { CAN } \\
\text { SLEEVE }\end{array}$ & $\begin{array}{l}12.0 \\
13.5 \\
16.0\end{array}$ & $\begin{array}{l}13.5 \\
14.0 \\
23.0\end{array}$ & $\begin{array}{l}13.5 \\
14.0 \\
23.0\end{array}$ & $\begin{array}{l}13.5 \\
14.9 \\
49.5\end{array}$ & $\begin{array}{l}13.5 \\
16.0 \\
49.5\end{array}$ & $\begin{array}{l}\text { STEEL } \\
\text { STEEL } \\
\text { INON }\end{array}$ & $\begin{array}{l}\text { STEEL } \\
\text { STEEL } \\
\text { IRON }\end{array}$ & $\begin{array}{l}9.9 \\
9.0\end{array}$ & $\begin{array}{l}\text { NONE } \\
\text { NONE } \\
\text { SAND-B }\end{array}$ \\
\hline$B 1.29 \mathrm{P}$ & $\begin{array}{l}\text { STAD } \\
\text { GAN } \\
\text { SLEEVE }\end{array}$ & $\begin{array}{l}12.0 \\
13.5 \\
16.0\end{array}$ & $\begin{array}{l}13.5 \\
14.0 \\
23.0\end{array}$ & $\begin{array}{l}13.5 \\
14.0 \\
23.0\end{array}$ & $\begin{array}{l}13.5 \\
10.0 \\
49.5\end{array}$ & $\begin{array}{r}13.5 \\
16.0 \\
49.5\end{array}$ & $\begin{array}{l}\text { STEEL } \\
\text { STEEL } \\
\text { I RON }\end{array}$ & $\begin{array}{l}\text { STEEL } \\
\text { STEEL } \\
\text { IRON }\end{array}$ & $\begin{array}{l}0.0 \\
0.0 \\
0.0\end{array}$ & $\begin{array}{l}\text { PONE } \\
\text { NONE } \\
\text { SAND-B }\end{array}$ \\
\hline
\end{tabular}


Table 4-4. (Continued)

\begin{tabular}{|c|c|c|c|c|c|c|c|c|c|c|}
\hline CONCEPT & ELEMENT & $\underset{\text { MATSI }}{\substack{\text { INS } \\
\text { MA }}}$ & $\begin{array}{l}\text { IDE } \\
\text { RIAL } \\
\text { OD }\end{array}$ & $\begin{array}{l}\text { OUTS IDE } \\
\text { MATERIAL } \\
\text { OD }\end{array}$ & $\begin{array}{c}\text { FILLER } \\
\text { OD }\end{array}$ & $\begin{array}{r}\text { GAP } \\
\text { OD }\end{array}$ & $\begin{array}{l}\text { INSIDE: } \\
\text { MATERIAL }\end{array}$ & $\begin{array}{l}\text { OUTS IDE } \\
\text { MATERINL: }\end{array}$ & $\begin{array}{c}\text { COATING } \\
\text { DELAY } \\
\text { (YRS) }\end{array}$ & FILLER \\
\hline $\mathrm{BE} \cdot \mathbb{1 N}$ & $\begin{array}{l}\text { CA STAE } \\
\text { CAN } \\
\text { SLEEVE }\end{array}$ & $\begin{array}{l}12.0 \\
13.5 \\
14.5\end{array}$ & $\begin{array}{l}13.5 \\
14.0 \\
15.0\end{array}$ & $\begin{array}{l}13.5 \\
14.0 \\
15.0\end{array}$ & $\begin{array}{l}13.5 \\
14.5 \\
48.0\end{array}$ & $\begin{array}{l}13.5 \\
14.5 \\
48.0\end{array}$ & $\begin{array}{l}\text { LEAD } \\
\text { 304SST } \\
\text { I HON }\end{array}$ & $\begin{array}{l}\text { LEAD } \\
304 S S T \\
\text { IRON }\end{array}$ & $\begin{array}{l}0.0 \\
0.0 \\
0.0\end{array}$ & $\begin{array}{l}\text { RONE } \\
\text { SAND-B } \\
\text { SAND-B }\end{array}$ \\
\hline BP. $.2 \mathrm{~N}$ & $\begin{array}{l}\text { CA STAB } \\
\text { CAN } \\
\text { SLEEVE }\end{array}$ & $\begin{array}{l}12.0 \\
13.5 \\
14.5\end{array}$ & $\begin{array}{l}13.5 \\
14.0 \\
21.5\end{array}$ & $\begin{array}{l}13.5 \\
14.0 \\
21.5\end{array}$ & $\begin{array}{l}13.5 \\
14.5 \\
48.0\end{array}$ & $\begin{array}{l}13.5 \\
14.5 \\
48.0\end{array}$ & $\begin{array}{l}\text { LEAD } \\
304 S S T \\
\text { IRON }\end{array}$ & $\begin{array}{l}\text { LEAD } \\
304 S S T \\
\text { IRON }\end{array}$ & $\begin{array}{l}0.0 \\
0.0 \\
0.0\end{array}$ & $\begin{array}{l}\text { NONE } \\
\text { SAND-B } \\
\text { SAND-B }\end{array}$ \\
\hline BE.3N & $\begin{array}{l}\text { CA STAB } \\
\text { CAN } \\
\text { SLEEVE }\end{array}$ & $\begin{array}{l}12.0 \\
13.5 \\
14.5\end{array}$ & $\begin{array}{l}13.5 \\
14.0 \\
26.5\end{array}$ & $\begin{array}{l}13.5 \\
14.0 \\
26.5\end{array}$ & $\begin{array}{l}13.5 \\
14.5 \\
46.0\end{array}$ & $\begin{array}{l}13.5 \\
14.5 \\
48.0\end{array}$ & $\begin{array}{l}\text { LEAD } \\
304 S S T \text {. } \\
\text { IRON }\end{array}$ & $\begin{array}{l}\text { LEAD } \\
\text { 394SST } \\
\text { IRON }\end{array}$ & $\begin{array}{l}0.0 \\
0.0 \\
0.0\end{array}$ & $\begin{array}{l}\text { NONE } \\
\text { SAND-B } \\
\text { SAND-B }\end{array}$ \\
\hline$B E .4 \mathbb{N}$ & $\begin{array}{l}\text { CA STAB } \\
\text { CAN } \\
\text { SLEEVE }\end{array}$ & $\begin{array}{l}12.0 \\
13.5 \\
14.5\end{array}$ & $\begin{array}{l}13.5 \\
14.0 \\
21.5\end{array}$ & $\begin{array}{l}13.5 \\
14.0 \\
21.6\end{array}$ & $\begin{array}{l}13.5 \\
14.5 \\
48.0\end{array}$ & $\begin{array}{l}13.5 \\
14.5 \\
88.0\end{array}$ & $\begin{array}{l}\text { LEAD } \\
3945 S T \\
\text { IRON }\end{array}$ & $\begin{array}{l}\text { LEAD } \\
3045 S T \\
\text { ZIRC }\end{array}$ & $\begin{array}{l}9.0 \\
0.0 \\
0.0\end{array}$ & $\begin{array}{l}\text { NONE } \\
\text { SAND-B } \\
\text { SAND-B }\end{array}$ \\
\hline $\mathrm{BE} .5 \mathrm{~N}$ & $\begin{array}{l}\text { CA STAB } \\
\text { CAR } \\
\text { SLEEVE }\end{array}$ & $\begin{array}{l}12.0 \\
13.5 \\
14.5\end{array}$ & $\begin{array}{l}13.5 \\
14.0 \\
21.5\end{array}$ & $\begin{array}{l}13.5 \\
14.0 \\
22.0\end{array}$ & $\begin{array}{l}13.5 \\
14.5 \\
48.0\end{array}$ & $\begin{array}{l}13.5 \\
14.5 \\
48.0\end{array}$ & $\begin{array}{l}\text { LCAD } \\
3045 S T \text {. } \\
\text { IRON }\end{array}$ & $\begin{array}{l}\text { LEAD } \\
\text { 304SST } \\
\text { ZIRC }\end{array}$ & $\begin{array}{l}0.0 \\
0.0 \\
0.0\end{array}$ & $\begin{array}{l}\text { NONE } \\
\text { SAND-B } \\
\text { SAND-B }\end{array}$ \\
\hline$B E .5 \%$ & $\begin{array}{l}\text { CA STAB } \\
\text { CAN } \\
\text { SLEEVE }\end{array}$ & $\begin{array}{l}12.0 \\
13.5 \\
14.5\end{array}$ & $\begin{array}{l}13.5 \\
14.0 \\
15.0\end{array}$ & $\begin{array}{l}13.5 \\
14.0 \\
15.3\end{array}$ & $\begin{array}{l}13.5 \\
14.5 \\
48.0\end{array}$ & $\begin{array}{l}13.5 \\
14.5 \\
43.0\end{array}$ & $\begin{array}{l}\text { LFAD } \\
3045 S T \\
\text { IRON }\end{array}$ & $\begin{array}{l}\text { LEAD } \\
3045 S T \\
\text { Z1RC }\end{array}$ & $\begin{array}{l}9.9 \\
0.0 \\
0.0\end{array}$ & $\begin{array}{l}\text { NONE } \\
\text { SAND-B } \\
\text { SAND-B }\end{array}$ \\
\hline $\mathrm{BE} .7 \mathrm{~N}$ & $\begin{array}{l}\text { CA STAB } \\
\text { CAN } \\
\text { SLEEVE }\end{array}$ & $\begin{array}{l}12.0 \\
13.5 \\
14.5\end{array}$ & $\begin{array}{l}13.5 \\
13.0 \\
26.5\end{array}$ & $\begin{array}{l}13.5 \\
14.8 \\
26.8\end{array}$ & $\begin{array}{l}13.5 \\
14.5 \\
48.0\end{array}$ & $\begin{array}{l}13.5 \\
14.5 \\
48.0\end{array}$ & $\begin{array}{l}\text { LEAD } \\
3045 S T \\
\text { IRON }\end{array}$ & $\begin{array}{l}\text { LEAD } \\
39.4 S T T \\
\text { ZIRC }\end{array}$ & $\begin{array}{l}0.0 \\
0.0 \\
0.0\end{array}$ & $\begin{array}{l}\text { NONE } \\
\text { SAND-B } \\
\text { SAND-B }\end{array}$ \\
\hline BE. $8 \mathrm{~N}$ & $\begin{array}{l}\text { CA STAB } \\
\text { CAN } \\
\text { SLEEVE }\end{array}$ & $\begin{array}{l}12.5 \\
13.5 \\
14.3\end{array}$ & $\begin{array}{l}13.5 \\
14.0 \\
15.0\end{array}$ & $\begin{array}{l}13.5 \\
14.0 \\
15.0\end{array}$ & $\begin{array}{l}13.5 \\
14.5 \\
48.0\end{array}$ & $\begin{array}{l}13.5 \\
14.5 \\
48.8\end{array}$ & $\begin{array}{l}\text { LEAD } \\
\text { ZIRC } \\
\text { INON }\end{array}$ & $\begin{array}{l}\text { LEAD } \\
\text { ZIRC } \\
\text { IRON }\end{array}$ & $\begin{array}{l}0.0 \\
0.0 \\
0.0\end{array}$ & $\begin{array}{l}\text { MONE } \\
\text { SAND-B } \\
\text { SAND-B }\end{array}$ \\
\hline $\mathrm{BE} .9 \mathrm{~F}$ & $\begin{array}{l}\text { CA STAB } \\
\text { CAN } \\
\text { SLEEVE }\end{array}$ & $\begin{array}{l}12.0 \\
13.5 \\
14.5\end{array}$ & $\begin{array}{l}13.5 \\
14.0 \\
31.5\end{array}$ & $\begin{array}{l}13.5 \\
14.8 \\
91.5\end{array}$ & $\begin{array}{l}13.5 \\
14.5 \\
48.9\end{array}$ & $\begin{array}{l}13.5 \\
19.5 \\
48.0\end{array}$ & $\begin{array}{l}\text { LEAD } \\
\text { ZIRC } \\
\text { IROR }\end{array}$ & $\begin{array}{l}\text { LEAD } \\
\text { ZIRC } \\
\text { IRON }\end{array}$ & $\begin{array}{l}0.0 \\
0.8 \\
0.0\end{array}$ & $\begin{array}{l}\text { RONE } \\
\text { SAND-B } \\
\text { SAID-B }\end{array}$ \\
\hline
\end{tabular}


Table 4-4. (Continued)

\begin{tabular}{|c|c|c|c|c|c|c|c|c|c|c|}
\hline CDEGERT & FLEMTET & $\begin{array}{c}\text { MVS } \\
\text { IRAT } \\
\text { ID }\end{array}$ & DI & $\begin{array}{l}\text { OUTS IDE } \\
\text { MATVRIAL } \\
\text { OD }\end{array}$ & $\begin{array}{l}\text { FILLEA } \\
\text { OD }\end{array}$ & $\begin{array}{l}\text { GAP } \\
\text { OD }\end{array}$ & $\begin{array}{l}\text { INS IDE! } \\
\text { MATERIAL }\end{array}$ & $\begin{array}{l}\text { OUTS IDE } \\
\text { MATERIALI }\end{array}$ & $\begin{array}{l}\text { COATING } \\
\text { DELAY } \\
\text { PTRS }\end{array}$ & 蒗 ILLER \\
\hline BE. 109 & $\begin{array}{l}\text { CA STAB } \\
\text { CAN } \\
\text { SLEEVY }\end{array}$ & $\begin{array}{l}12.5 \\
13.5 \\
11.5 \\
14.5\end{array}$ & 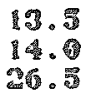 & $\begin{array}{l}13.5 \\
1.5 .0 \\
26.5\end{array}$ & 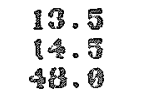 & $\begin{array}{l}13.5 \\
13.57 \\
48.07\end{array}$ & $\begin{array}{l}\text { LEAD } \\
\text { ZIRC } \\
\text { IRON }\end{array}$ & $\begin{array}{l}\text { LEAD } \\
\text { ZURC } \\
\text { IRON }\end{array}$ & $\begin{array}{l}0.9 \\
0.0 \\
0.0\end{array}$ & $\begin{array}{l}\text { MONE } \\
\text { SAND-B } \\
\text { SAND-B }\end{array}$ \\
\hline BE。1 & $\begin{array}{l}\text { CA STA } \\
\text { CAR } \\
\text { SLECVE }\end{array}$ & $\begin{array}{l}18.9 \\
12.5 \\
14.5\end{array}$ & $\begin{array}{l}13.5 \\
13.9 \\
21.9\end{array}$ & $\begin{array}{l}13.5 \\
19.6 \\
31.6\end{array}$ & $\begin{array}{l}13.5 \\
14.5 \\
40.0\end{array}$ & $\begin{array}{l}13.5 \\
14.5 \\
48.0\end{array}$ & $\begin{array}{l}\text { LEAD } \\
\text { ZIRC } \\
\text { IRON }\end{array}$ & $\begin{array}{l}\text { LEAD } \\
\text { ZIRC } \\
\text { ZIRC }\end{array}$ & $\begin{array}{l}0.9 \\
0.0 \\
0.0\end{array}$ & $\begin{array}{l}\text { WONE } \\
\text { SAND-B } \\
\text { SAND-B }\end{array}$ \\
\hline 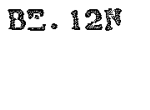 & $\begin{array}{l}\text { AR STAB } \\
\text { CAR } \\
\text { SLEEVE }\end{array}$ & $\begin{array}{l}12.0 \\
15.9 \\
140.5\end{array}$ & 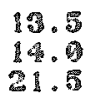 & $\begin{array}{l}13.5 \\
14.0 \\
22.9\end{array}$ & $\begin{array}{l}13.5 \\
14.5 \\
48.5\end{array}$ & $\begin{array}{l}13.5 \\
13.5 \\
48.9\end{array}$ & $\begin{array}{l}\text { LEAD } \\
\text { ZIRC } \\
\text { MRON }\end{array}$ & $\begin{array}{l}\text { LEAD } \\
\text { ZIRG } \\
\text { ZIRC }\end{array}$ & $\begin{array}{l}0.0 \\
9.0 \\
9.6\end{array}$ & $\begin{array}{l}\text { WORPE } \\
\text { AND-B } \\
3 A N D-B\end{array}$ \\
\hline DT. $13 \%$ & $\begin{array}{l}\text { CA STA } \\
\text { CAN } \\
\text { SLERVE }\end{array}$ & $\begin{array}{l}12.0 \\
12.5 \\
14.5\end{array}$ & $\begin{array}{l}13.5 \\
1460 \\
15.0\end{array}$ & $\begin{array}{l}13.5 \\
14.0 \\
15.3\end{array}$ & $\begin{array}{l}193.5 \\
14.5 \\
48.0\end{array}$ & $\begin{array}{l}13.5 \\
14.5 \\
48.0\end{array}$ & $\begin{array}{l}\text { CRAD } \\
\text { ZIRC } \\
\text { IROA }\end{array}$ & $\begin{array}{l}\text { LEAD } \\
\text { ZIREC } \\
\text { ZIRC }\end{array}$ & $\begin{array}{l}0.0 \\
0.0\end{array}$ & $\begin{array}{l}\text { ROAR } \\
\text { SAND-B } \\
\text { SAND-D }\end{array}$ \\
\hline BED. 1 A 17 & $\begin{array}{l}\text { GA STAS } \\
\text { CAR } \\
\text { WEREVR }\end{array}$ & $\begin{array}{l}12.0 \\
13.5 \\
160.5\end{array}$ & $\begin{array}{l}13.5 \\
13.0 \\
26.5 \\
26.5\end{array}$ & $\begin{array}{l}13.5 \\
18.5 \\
26.6\end{array}$ & $\begin{array}{l}13.5 \\
1.5 .5 \\
40.5\end{array}$ & $\begin{array}{l}13.5 \\
14.35 \\
440.07\end{array}$ & $\begin{array}{l}\text { CEAD } \\
\text { ZIRE } \\
\text { IRON }\end{array}$ & $\begin{array}{l}\text { LEAD } \\
\text { ZIRC } \\
\text { ZIRC }\end{array}$ & $\begin{array}{l}0.0 \\
0.0\end{array}$ & $\begin{array}{l}\text { SOEE } \\
\text { SADD- } \\
3 \mathrm{AND}-\mathrm{D}\end{array}$ \\
\hline 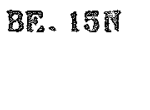 & $\begin{array}{l}\text { GA STAB } \\
\text { EAN } \\
\text { SLEEVE }\end{array}$ & $\begin{array}{l}12.6 \\
13.5 \\
14.5\end{array}$ & $\begin{array}{l}13.5 \\
13.0 \\
15.0\end{array}$ & $\begin{array}{l}13.5 \\
14.9 \\
15.9\end{array}$ & $\begin{array}{l}13.5 \\
1.5 .5 \\
49.0\end{array}$ & $\begin{array}{l}13.5 \\
19.5 \\
48.0\end{array}$ & $\begin{array}{l}\text { LEAD } \\
\text { INCONEL } \\
\text { IRON }\end{array}$ & $\begin{array}{l}\text { LEAD } \\
\text { INCONELI } \\
\text { RON }\end{array}$ & $\begin{array}{l}0.0 \\
9.0 \\
0.0\end{array}$ & $\begin{array}{l}\text { HONE } \\
S A N D-1 \\
S A D D-1\end{array}$ \\
\hline EE.16N & $\begin{array}{l}\text { CA STAB } \\
\text { CSII } \\
\text { SLEEVE }\end{array}$ & $\begin{array}{l}12.0 \\
12.5 \\
14.5\end{array}$ & $\begin{array}{l}13.5 \\
11.0 \\
21.5\end{array}$ & $\begin{array}{l}13.5 \\
1.9 \\
21.5\end{array}$ & $\begin{array}{l}13.5 \\
14.5 \\
45.9 \\
450\end{array}$ & $\begin{array}{l}13.3 \\
14.5 \\
48.0\end{array}$ & $\begin{array}{l}\text { LEAD } \\
\text { INGCOREL } \\
\text { IRON }\end{array}$ & $\begin{array}{l}\text { LEAD } \\
\text { INCOREL: } \\
\text { IRON }\end{array}$ & $\begin{array}{l}0.0 \\
0.0\end{array}$ & $\begin{array}{l}\text { MONE } \\
\text { SARD- } \\
\text { SAND- }\end{array}$ \\
\hline BE. 187 & $\begin{array}{l}\text { CA STAB } \\
\text { TAR } \\
\text { SLEEYTE }\end{array}$ & $\begin{array}{l}12.0 \\
13.5 \\
13.5\end{array}$ & 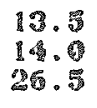 & $\begin{array}{l}13.5 \\
13.9 \\
26.5\end{array}$ & $\begin{array}{l}13.5 \\
13.5 \\
48.5\end{array}$ & $\begin{array}{l}13.5 \\
13.5 \\
48.0\end{array}$ & $\begin{array}{l}\text { LEAD } \\
\text { INCONEL } \\
\text { IROP }\end{array}$ & $\begin{array}{l}\text { LEAD } \\
\text { INCONELR } \\
\text { IRON }\end{array}$ & $\begin{array}{l}9.0 \\
0.0 \\
0.0\end{array}$ & $\begin{array}{l}\text { PONE } \\
\text { SARD-B } \\
\text { SARD-B }\end{array}$ \\
\hline $\mathrm{AE} \cdot 18 \mathrm{~V}$ & $\begin{array}{l}\text { CA STAB } \\
\text { CANE } \\
\text { SLEEVIE }\end{array}$ & $\begin{array}{l}13.0 \\
13.5 \\
14.5\end{array}$ & $\begin{array}{l}19.5 \\
14.9 \\
21.5\end{array}$ & $\begin{array}{l}12.5 \\
14.0 \\
21.6\end{array}$ & $\begin{array}{l}13.5 \\
10.5 \\
48.9\end{array}$ & $\begin{array}{l}13.5 \\
19.5 \\
48.0\end{array}$ & $\begin{array}{l}\text { LEAD } \\
\text { IHCONEL } \\
\text { MON }\end{array}$ & $\begin{array}{l}\text { LEAD } \\
\text { INCONEL: } \\
\text { ZIRC }\end{array}$ & $\begin{array}{l}0.0 \\
0.0 \\
0.0\end{array}$ & $\begin{array}{l}\text { NONE } \\
\text { SAND-B } \\
\text { SAND-B }\end{array}$ \\
\hline
\end{tabular}


Table 4-4. (Continued)

\begin{tabular}{|c|c|c|c|c|c|c|c|c|c|c|}
\hline CDHCET & ELEMTERT & $\begin{array}{l}\text { MIS } \\
\text { MATER } \\
\text { ID }\end{array}$ & $\begin{array}{l}\text { IDE } \\
\text { MIAL } \\
\text { OD }\end{array}$ & $\begin{array}{c}\text { OUTSIDE } \\
\text { MATRIAAL } \\
\text { OD }\end{array}$ & $\begin{array}{c}\text { PICLER } \\
\text { OD }\end{array}$ & $\begin{array}{r}\text { GAP } \\
\text { OD }\end{array}$ & $\begin{array}{l}\text { IPS IDE } \\
\text { MATEMIAL }\end{array}$ & $\begin{array}{l}\text { OUTSIDE } \\
\text { RATERIALI }\end{array}$ & $\begin{array}{l}\text { COATING } \\
\text { DELAY } \\
\text { (YRS) }\end{array}$ & PLLER \\
\hline Be. $19 \mathrm{~N}$ & $\begin{array}{l}\text { CA STAB } \\
\text { CAN } \\
\text { SLETER }\end{array}$ & $\begin{array}{l}13.0 \\
13.5 \\
14.5\end{array}$ & $\begin{array}{l}13.9 \\
16.0 \\
21.93\end{array}$ & $\begin{array}{l}13.5 \\
13.5 \\
122.0 \\
212.0\end{array}$ & $\begin{array}{l}19.5 \\
14.5 \\
48.9\end{array}$ & 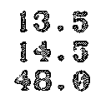 & $\begin{array}{l}\text { LEAD } \\
\text { INCONEL } \\
\text { IRON }\end{array}$ & $\begin{array}{l}\text { LEAD } \\
\text { MNCONELI } \\
\text { ZIRC }\end{array}$ & $\begin{array}{l}0.0 \\
0.0 \\
0.0\end{array}$ & $\begin{array}{l}\text { NOPE } \\
\text { SARD-D } \\
\text { SAND-B }\end{array}$ \\
\hline D蕰.20N & $\begin{array}{l}\text { CA STAB } \\
\text { CAR } \\
\text { SLEEVER }\end{array}$ & $\begin{array}{l}12.0 \\
13.5 \\
13.53\end{array}$ & $\begin{array}{l}13.5 \\
14.0 \\
13.0\end{array}$ & $\begin{array}{l}13.5 \\
14.8 \\
119.8\end{array}$ & 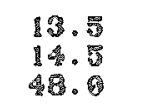 & $\begin{array}{l}13.5 \\
19.5 \\
148.5\end{array}$ & $\begin{array}{l}\text { LRAD } \\
\text { IICONEL } \\
\text { IRON }\end{array}$ & $\begin{array}{l}\text { LEAD } \\
\text { INCONELi } \\
\text { ZIRC }\end{array}$ & $\begin{array}{l}0.0 \\
0.0\end{array}$ & $\begin{array}{l}\text { RONE } \\
\text { SAND-B } \\
\text { SAND-B }\end{array}$ \\
\hline BR.2IN & $\begin{array}{l}\text { CA \$TAB } \\
\text { CAN } \\
\text { SLEEVE }\end{array}$ & $\begin{array}{l}12.0 \\
12.5 \\
1.5\end{array}$ & $\begin{array}{l}13.5 \\
14.0 \\
24.5 \\
25\end{array}$ & $\begin{array}{l}13.5 \\
13.08 \\
196.8\end{array}$ & 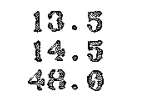 & 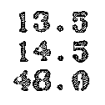 & $\begin{array}{l}\text { LEAD } \\
\text { IPCONEL } \\
\text { POON }\end{array}$ & $\begin{array}{l}\text { LEAD } \\
\text { BNCONEL } \\
\text { ZIRC }\end{array}$ & 0.0 & $\begin{array}{l}\text { PONE } \\
\text { SAND-D } \\
\text { SAND-D }\end{array}$ \\
\hline DE. 22R & $\begin{array}{l}\text { CA STAD } \\
\text { CAR } \\
\text { SLLRWT }\end{array}$ & $\begin{array}{l}12.0 \\
182.5 \\
1.62 .5\end{array}$ & $\begin{array}{l}13.5 \\
13.5 \\
11.5 \\
31.5\end{array}$ & $\begin{array}{l}19.7 \\
19.9 \\
21.5 \\
21.5\end{array}$ & $\begin{array}{l}13.5 \\
16.5 \\
40.0\end{array}$ & 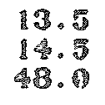 & $\begin{array}{l}\text { LEAD } \\
\text { ZINC } \\
\text { IRON }\end{array}$ & $\begin{array}{l}\text { LEAD } \\
2112 C \\
\text { IRON }\end{array}$ & $\begin{array}{l}0.0 \\
0.0 \\
0.0\end{array}$ & $\begin{array}{l}\text { WONE } \\
\text { SAND-B } \\
\text { SAND-D }\end{array}$ \\
\hline DE. 231 & $\begin{array}{l}\text { CA BTAB } \\
\text { CAR } \\
\text { SLEVVE }\end{array}$ & $\begin{array}{l}12.0 \\
13.5 \\
13.5\end{array}$ & $\begin{array}{l}13.5 \\
14.0 \\
26.5 \\
26.9\end{array}$ & $\begin{array}{l}13.5 \\
13.5 \\
26.5\end{array}$ & $\begin{array}{l}13.5 \\
13.5 \\
48.9\end{array}$ & $\begin{array}{l}13.5 \\
14.5 \\
40.0\end{array}$ & $\begin{array}{l}\text { UEAD } \\
\text { ZIRC } \\
\text { IRON }\end{array}$ & $\begin{array}{l}\text { LEAD } \\
\text { ZIRC } \\
\text { IRON }\end{array}$ & $\begin{array}{l}0.03 \\
0.00\end{array}$ & $\begin{array}{l}\text { PONO } \\
\text { SAND-R } \\
\text { SAND-D }\end{array}$ \\
\hline BE.24:4II & $\begin{array}{l}\text { PA STAR } \\
\text { CAR } \\
\text { SLEEVE }\end{array}$ & $\begin{array}{l}12.0 \\
13.5 \\
13.5\end{array}$ & $\begin{array}{l}13.9 \\
13.0 \\
21.5\end{array}$ & $\begin{array}{l}13.5 \\
19.60 \\
21.6 \\
21.6\end{array}$ & 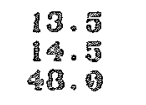 & $\begin{array}{l}13.5 \\
19.5 \\
48.5 \\
43.5\end{array}$ & $\begin{array}{l}\text { LEADE } \\
\text { ZIIRC } \\
\text { BRON }\end{array}$ & $\begin{array}{l}\text { LEAD } \\
\text { ZIRE } \\
\text { 2IRC }\end{array}$ & $\begin{array}{l}0.0 \\
0.0 \\
0.0\end{array}$ & $\begin{array}{l}\text { MONE } \\
\text { SAND-D } \\
\text { SAND-B }\end{array}$ \\
\hline BR.2\% & $\begin{array}{l}\text { CA STTE } \\
\text { CAN } \\
\text { SLEEVE }\end{array}$ & $\begin{array}{l}12.0 \\
13.5 \\
14.5\end{array}$ & $\begin{array}{l}13.5 \\
14.5 \\
11.5 \\
81.5\end{array}$ & $\begin{array}{l}13.7 \\
14.7 \\
23.0\end{array}$ & $\begin{array}{l}13.5 \\
14.5 \\
48.5\end{array}$ & $\begin{array}{l}13.5 \\
14 . \\
48.13\end{array}$ & $\begin{array}{l}\text { LUAD } \\
\text { ZIRC } \\
\text { IROR }\end{array}$ & $\begin{array}{l}\text { LEAD } \\
2 I R C \\
2 I M C\end{array}$ & $\begin{array}{l}9.0 \\
0.0 \\
0.0\end{array}$ & $\begin{array}{l}\text { MONRE } \\
S A N D-B \\
S A N D-D\end{array}$ \\
\hline BR. & $\begin{array}{l}\text { CA STAB } \\
\text { CAN } \\
\text { SLEEVE }\end{array}$ & $\begin{array}{l}12.0 \\
13.5 \\
13.5\end{array}$ & $\begin{array}{l}13.5 \\
14.0 \\
21.9\end{array}$ & $\begin{array}{l}13.5 \\
19.5 \\
21.5\end{array}$ & 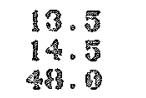 & $\begin{array}{l}13.5 \\
14.5 \\
40.0\end{array}$ & $\begin{array}{l}\text { LEAD } \\
\text { 304SST } \\
\text { IRUM }\end{array}$ & $\begin{array}{l}\text { LEAD } \\
\text { 304SST } \\
\text { IRON }\end{array}$ & $\begin{array}{l}9.6 \\
9.0 \\
9.6\end{array}$ & $\begin{array}{l}\text { MONE } \\
\text { BENT } \\
\text { BENT }\end{array}$ \\
\hline$E .38 \pi$ & $\begin{array}{l}\text { CA STAB } \\
\text { CAM } \\
\text { SLEEYE }\end{array}$ & $\begin{array}{l}12.0 \\
13.3 \\
14.5\end{array}$ & $\begin{array}{l}13.5 \\
14.0 \\
21.5\end{array}$ & $\begin{array}{l}13.5 \\
16.0 \\
21.6\end{array}$ & $\begin{array}{l}13.5 \\
14.5 \\
14.5 \\
48.0\end{array}$ & $\begin{array}{l}13.9 \\
13.5 \\
48.98\end{array}$ & $\begin{array}{l}\text { LFAD } \\
3945 S T \\
\text { IRON }\end{array}$ & $\begin{array}{l}\text { LEAD } \\
3045 S T \\
\text { ZIRC }\end{array}$ & $\begin{array}{l}0.0 \\
0.0 \\
0.0\end{array}$ & $\begin{array}{l}\text { NORE } \\
\text { BERT } \\
\text { BENT }\end{array}$ \\
\hline
\end{tabular}


Table 4-4. (Continued)

\begin{tabular}{|c|c|c|c|c|c|c|c|c|c|c|}
\hline CONCEPT & ELENTIT & $\begin{array}{l}\text { 1\%SI } \\
\text { MATES } \\
\text { ID }\end{array}$ & $\begin{array}{l}\text { IDE } \\
\text { IIAL } \\
\text { OD }\end{array}$ & $\begin{array}{c}\text { OUTS IDL } \\
\text { RATRRIAL } \\
\text { OD }\end{array}$ & $\begin{array}{c}\text { FILLER } \\
\text { OD }\end{array}$ & $\begin{array}{l}\text { GP } \\
\text { OD }\end{array}$ & $\begin{array}{r}\text { INSIDEL } \\
\text { WATERIAL }\end{array}$ & $\begin{array}{l}\text { OUTS IDE } \\
\text { IATRRIAL }\end{array}$ & $\begin{array}{l}\text { COATING } \\
\text { DELAY } \\
\text { (MRS) }\end{array}$ & FILLER \\
\hline $\mathrm{BE} .28 \mathrm{~N}$ & $\begin{array}{l}\text { CA STAB } \\
\text { CAR } \\
\text { SIEEVE }\end{array}$ & $\begin{array}{l}12.0 \\
13.5 \\
14.5\end{array}$ & $\begin{array}{l}19.5 \\
14.0 \\
21.5\end{array}$ & $\begin{array}{l}13.5 \\
14.5 \\
21.5\end{array}$ & $\begin{array}{l}13.5 \\
14.5 \\
48.0\end{array}$ & $\begin{array}{r}13.5 \\
14.5 \\
48.0\end{array}$ & $\begin{array}{l}\text { LEAD } \\
3945 S T \\
\text { IRON }\end{array}$ & $\begin{array}{l}\text { LEAD } \\
\text { 304SST } \\
\text { IRON }\end{array}$ & 9.0 & $\begin{array}{l}\text { NONE } \\
\text { CLINO } \\
\text { CLINO }\end{array}$ \\
\hline Br. 29 N & $\begin{array}{l}\text { CA STAB } \\
\text { CAN } \\
\text { SLEEVE }\end{array}$ & $\begin{array}{l}12.0 \\
13.5 \\
14.5\end{array}$ & $\begin{array}{l}13.5 \\
14.0 \\
21.5\end{array}$ & $\begin{array}{l}13.5 \\
14.0 \\
21.6\end{array}$ & $\begin{array}{l}13.5 \\
14.5 \\
48.0\end{array}$ & $\begin{array}{l}13.5 \\
13.5 \\
48.0\end{array}$ & $\begin{array}{l}\text { LEAD } \\
\text { 304SST } \\
\text { IRON }\end{array}$ & $\begin{array}{l}\text { LEAD } \\
\text { 304SST } \\
\text { ZIRC }\end{array}$ & $\begin{array}{l}0.0 \\
0.0 \\
0.0\end{array}$ & $\begin{array}{l}\text { NONE } \\
\text { CLINO } \\
\text { CLINO }\end{array}$ \\
\hline BE. $30 \mathrm{~N}$ & $\begin{array}{l}\text { CA STAB } \\
\text { CAN } \\
\text { SLEEVE }\end{array}$ & $\begin{array}{l}12.0 \\
13.5 \\
14.5\end{array}$ & $\begin{array}{l}13.5 \\
14.0 \\
15.0\end{array}$ & $\begin{array}{l}13.5 \\
14.5 \\
15.0\end{array}$ & $\begin{array}{l}13.5 \\
14.5 \\
20.0\end{array}$ & $\begin{array}{l}13.5 \\
14.5 \\
20.0\end{array}$ & $\begin{array}{l}\text { LEAD } \\
304 S S T \\
\text { InON }\end{array}$ & $\begin{array}{l}\text { LEAD } \\
\text { 204SST } \\
\text { IRON }\end{array}$ & $\begin{array}{l}9.9 \\
0.9 \\
0.0\end{array}$ & $\begin{array}{l}\text { NONE } \\
\text { SAND-B } \\
\text { SAND-B }\end{array}$ \\
\hline BE. 31N & $\begin{array}{l}\text { CA STAB } \\
\text { CAN } \\
\text { SLEEVE }\end{array}$ & $\begin{array}{l}12.0 \\
13.5 \\
14.5\end{array}$ & $\begin{array}{l}13.5 \\
14.0 \\
15.0\end{array}$ & $\begin{array}{l}13.7 \\
14.0 \\
15.0\end{array}$ & $\begin{array}{l}13.5 \\
14.5 \\
36.0\end{array}$ & $\begin{array}{l}13.5 \\
14.5 \\
36.0\end{array}$ & $\begin{array}{l}\text { LEAD } \\
\text { 304SST } \\
\text { I HON }\end{array}$ & $\begin{array}{l}\text { LEAD } \\
2045 S T \\
\text { IRON }\end{array}$ & $\begin{array}{l}0.0 \\
0.0 \\
0.0\end{array}$ & $\begin{array}{l}\text { NONE } \\
\text { SAND-B } \\
\text { SAND-B }\end{array}$ \\
\hline BE. 32N & $\begin{array}{l}\text { CA STAB } \\
\text { CAR } \\
\text { SLEEVE }\end{array}$ & $\begin{array}{l}12.0 \\
13.5 \\
14.5\end{array}$ & $\begin{array}{l}13.5 \\
14.0 \\
15.0\end{array}$ & $\begin{array}{l}13.5 \\
13.0 \\
15.3\end{array}$ & $\begin{array}{l}13.5 \\
14.5 \\
20.0\end{array}$ & $\begin{array}{l}13.5 \\
14.5 \\
20.0\end{array}$ & $\begin{array}{l}\text { LEAD } \\
3045 S T \\
\text { IRON }\end{array}$ & $\begin{array}{l}\text { LEAD } \\
394 S S T \\
\text { ZIRC }\end{array}$ & $\begin{array}{l}0.0 \\
0.9 \\
0.0\end{array}$ & $\begin{array}{l}\text { NONE } \\
\text { SAND-B } \\
\text { SAND-B }\end{array}$ \\
\hline BE. 33N & $\begin{array}{l}\text { CA STAB } \\
\text { CAN } \\
\text { SLEEVIS }\end{array}$ & $\begin{array}{l}12.0 \\
13.5 \\
14.5\end{array}$ & $\begin{array}{l}13.5 \\
14.5 \\
15.6\end{array}$ & $\begin{array}{l}13.5 \\
14.9 \\
15.3\end{array}$ & $\begin{array}{l}13.5 \\
14.5 \\
36.0\end{array}$ & $\begin{array}{l}13.5 \\
14.5 \\
36.0\end{array}$ & $\begin{array}{l}\text { LFAD } \\
30455 T \\
\text { IFON }\end{array}$ & $\begin{array}{l}\text { LEAD } \\
30455 T \\
21 R C\end{array}$ & $\begin{array}{l}0.0 \\
0.0 \\
0.0\end{array}$ & $\begin{array}{l}\text { NONE } \\
\text { SAND-B } \\
\text { SAND-B }\end{array}$ \\
\hline$B E .34 N$ & $\begin{array}{l}\text { CA STAB } \\
\text { CAM } \\
\text { SLECVE }\end{array}$ & $\begin{array}{l}12.0 \\
13.5 \\
14.5\end{array}$ & $\begin{array}{l}13.5 \\
14.0 \\
21.5\end{array}$ & $\begin{array}{l}13.5 \\
14.5 \\
21.5\end{array}$ & $\begin{array}{l}13.5 \\
14.5 \\
48.0\end{array}$ & $\begin{array}{l}13.5 \\
14.5 \\
40.0\end{array}$ & $\begin{array}{l}\text { LEAD } \\
30455 T \\
I R O N\end{array}$ & $\begin{array}{l}\text { LEAD } \\
39455 T \\
\text { IRON }\end{array}$ & $\begin{array}{r}9.0 \\
9.0 \\
160.0\end{array}$ & $\begin{array}{l}\text { NONL } \\
\text { SAND-D } \\
S A N D-B\end{array}$ \\
\hline BE. 35 T & $\begin{array}{l}\text { CA STAB } \\
\text { CAN } \\
\text { SLEEVE }\end{array}$ & $\begin{array}{l}12.0 \\
13.5 \\
14.5\end{array}$ & $\begin{array}{l}13.5 \\
14.0 \\
21.5\end{array}$ & $\begin{array}{l}19.5 \\
14.0 \\
21.6\end{array}$ & $\begin{array}{l}13.5 \\
14.5 \\
48.0\end{array}$ & $\begin{array}{l}13.5 \\
14.5 \\
48.0\end{array}$ & $\begin{array}{l}\text { LEAD } \\
3045 S T \\
\text { RON }\end{array}$ & $\begin{array}{l}\text { LEAD } \\
30 \text { SST } \\
\text { Z1RC }\end{array}$ & $\begin{array}{r}9.9 \\
6.0 \\
109.9\end{array}$ & $\begin{array}{l}\text { NONE } \\
\text { SAND-B } \\
\$ A N D-B\end{array}$ \\
\hline BE.36 & $\begin{array}{l}\text { CA STAB } \\
\text { CAN } \\
\text { SLEEVE }\end{array}$ & $\begin{array}{l}12.0 \\
13.5 \\
14.3\end{array}$ & $\begin{array}{l}13.5 \\
13.5 \\
21.3\end{array}$ & $\begin{array}{l}13.5 \\
14.0 \\
21.9\end{array}$ & $\begin{array}{l}13.5 \\
13.0 \\
47.8\end{array}$ & $\begin{array}{l}13.5 \\
14.3 \\
47.8\end{array}$ & $\begin{array}{l}\text { LEAD } \\
\text { ZIRC } \\
\text { IRON }\end{array}$ & $\begin{array}{l}\text { LEAD } \\
\text { ZIRC } \\
\text { IRON }\end{array}$ & $\begin{array}{l}1.0 \\
0.9 \\
0.0\end{array}$ & $\begin{array}{l}\text { PONE } \\
\text { NONE } \\
\text { SAND-B }\end{array}$ \\
\hline
\end{tabular}


Table 4-4. (Continued)

\begin{tabular}{|c|c|c|c|c|c|c|c|c|c|c|}
\hline CORCEPT & ELEMENT & $\begin{array}{c}\text { IISS I } \\
\text { MATER } \\
\text { III }\end{array}$ & $\begin{array}{l}\text { IDE } \\
\text { IAL } \\
O D\end{array}$ & $\begin{array}{l}\text { OUTS IDE } \\
\text { MATERIAL } \\
\text { OD }\end{array}$ & $\begin{array}{c}\text { FILLER } \\
\text { OD }\end{array}$ & $\begin{array}{l}\text { GAP } \\
O D\end{array}$ & $\begin{array}{r}\text { INSIDE: } \\
\text { MATERIAL }\end{array}$ & $\begin{array}{c}\text { OUTSIDE } \\
\text { MATERIALI }\end{array}$ & $\begin{array}{l}\text { COATING } \\
\text { DELAY } \\
\text { (YRS) }\end{array}$ & FILLER \\
\hline D望.37 & $\begin{array}{l}\text { CA STAB } \\
\text { CAN } \\
\text { SLEEVE }\end{array}$ & $\begin{array}{l}12.0 \\
13.5 \\
14.5\end{array}$ & $\begin{array}{l}13.5 \\
13.0 \\
21.5\end{array}$ & $\begin{array}{l}13.5 \\
14.0 \\
21.5\end{array}$ & $\begin{array}{l}13.5 \\
14.0 \\
411.0\end{array}$ & $\begin{array}{l}13.5 \\
14.5 \\
48.0\end{array}$ & $\begin{array}{l}\text { LEAD } \\
\text { ZIRC } \\
\text { IRON }\end{array}$ & $\begin{array}{l}\text { LEAD } \\
\text { ZIRC } \\
\text { InON }\end{array}$ & $\begin{array}{l}0.0 \\
0.0 \\
0.0\end{array}$ & $\begin{array}{l}\text { NONE } \\
\text { NONE } \\
\text { SAND-B }\end{array}$ \\
\hline DE.38N & $\begin{array}{l}\text { CA STAB } \\
\text { CAR } \\
\text { SLEEVE }\end{array}$ & $\begin{array}{l}12.0 \\
13.5 \\
15.0\end{array}$ & $\begin{array}{l}13.5 \\
14.0 \\
22.0\end{array}$ & $\begin{array}{l}13.5 \\
14.0 \\
22.0\end{array}$ & $\begin{array}{l}13.5 \\
14.0 \\
48.5\end{array}$ & $\begin{array}{l}13.5 \\
15.0 \\
48.5\end{array}$ & $\begin{array}{l}\text { LEAD } \\
\text { ZIRC } \\
\text { IRON }\end{array}$ & $\begin{array}{l}\text { LEAD } \\
\text { ZIRC } \\
\text { IRON }\end{array}$ & $\begin{array}{l}0.0 \\
0.0 \\
0.0\end{array}$ & $\begin{array}{l}\text { RORE } \\
\text { NONE } \\
\text { SAND-B }\end{array}$ \\
\hline BE. $39 N$ & $\begin{array}{l}\text { CA STAB } \\
\text { CAN } \\
\text { SLEEVE }\end{array}$ & $\begin{array}{l}12.0 \\
13.5 \\
16.9\end{array}$ & $\begin{array}{l}13.5 \\
14.0 \\
23.0\end{array}$ & $\begin{array}{l}13.5 \\
14.0 \\
23.0\end{array}$ & $\begin{array}{l}13.5 \\
13.0 \\
49.5\end{array}$ & $\begin{array}{l}13.5 \\
16.0 \\
49.5\end{array}$ & $\begin{array}{l}\text { LEAD } \\
\text { ZIRC } \\
\text { IMON }\end{array}$ & $\begin{array}{l}\text { LEAD } \\
\text { ZIRC } \\
\text { IRON }\end{array}$ & $\begin{array}{l}0.0 \\
0.0 \\
0.0\end{array}$ & $\begin{array}{l}\text { NONE } \\
\text { NONE } \\
\text { SAND-B }\end{array}$ \\
\hline C. 7 & $\begin{array}{l}\text { STAB } \\
\text { CAN } \\
\text { O PACK }\end{array}$ & $\begin{array}{l}12.0 \\
13.5 \\
16.0\end{array}$ & $\begin{array}{l}12.0 \\
15.5 \\
18.0\end{array}$ & $\begin{array}{l}12.0 \\
15.5 \\
10.0\end{array}$ & $\begin{array}{l}12.0 \\
16.0 \\
40.0\end{array}$ & $\begin{array}{l}13.5 \\
16.0 \\
40.0\end{array}$ & $\begin{array}{l}\text { HELIUM. } \\
\text { ZIRC } \\
\text { ZIRC }\end{array}$ & $\begin{array}{l}\text { HELIUM } \\
\text { ZIRC } \\
\text { ZIRC }\end{array}$ & $\begin{array}{l}0.0 \\
0.0 \\
0.0\end{array}$ & $\begin{array}{l}\text { NONE } \\
\text { SAND-B } \\
\text { SAND-B }\end{array}$ \\
\hline C1.1 & $\begin{array}{l}\text { STAB } \\
\text { CAN } \\
\text { 0 PACK } \\
\text { SLEEVE }\end{array}$ & $\begin{array}{l}12.0 \\
13.5 \\
14.5 \\
15.5\end{array}$ & $\begin{array}{l}13.5 \\
1.40 \\
15.0 \\
22.5\end{array}$ & $\begin{array}{l}13.5 \\
14.9 \\
15.0 \\
22.5\end{array}$ & $\begin{array}{l}13.5 \\
13.5 \\
15.5 \\
45.0\end{array}$ & $\begin{array}{l}13.5 \\
14.5 \\
15.5 \\
45.5\end{array}$ & $\begin{array}{l}\text { INCONEL } \\
\text { INCONEL } \\
\text { 304SST } \\
\text { INON }\end{array}$ & $\begin{array}{l}\text { INCONELI } \\
\text { INCONEL } \\
304 S S T \\
\text { IRON }\end{array}$ & $\begin{array}{l}0.0 \\
0.0 \\
0.0 \\
0.0\end{array}$ & $\begin{array}{l}\text { NONE } \\
\text { SAND-B } \\
\text { SAND-B } \\
\text { SAND-B }\end{array}$ \\
\hline C1. 1 & $\begin{array}{l}\text { STAB } \\
\text { CAN } \\
\text { O PACK } \\
\text { SLEEVE }\end{array}$ & $\begin{array}{l}12.0 \\
13.5 \\
13.5 \\
15.5\end{array}$ & $\begin{array}{l}12.0 \\
11.0 \\
15.0 \\
28.5\end{array}$ & $\begin{array}{l}12.0 \\
14.0 \\
15.0 \\
22.5\end{array}$ & $\begin{array}{l}12.0 \\
14.5 \\
15.5 \\
45.0\end{array}$ & $\begin{array}{l}13.5 \\
14.5 \\
15.5 \\
45.0\end{array}$ & $\begin{array}{l}\text { HELIIM. } \\
\text { IMCONEL } \\
304 S S T \\
\text { IRON }\end{array}$ & $\begin{array}{l}\text { HEL I UM } \\
\text { INCONEL } \\
394 S S T \\
\text { IRON }\end{array}$ & $\begin{array}{l}0.0 \\
0.0 \\
0.9 \\
0.0\end{array}$ & $\begin{array}{l}\text { MONE } \\
\text { SAND-B } \\
\text { SAND-B } \\
\text { SAND-B }\end{array}$ \\
\hline C 1.6 & $\begin{array}{l}\text { STAB } \\
\text { CAN } \\
\text { O PACK } \\
\text { SLEEVE }\end{array}$ & $\begin{array}{l}12.9 \\
13.5 \\
14.5 \\
13.5\end{array}$ & $\begin{array}{l}13.5 \\
14.0 \\
15.0 \\
16.5\end{array}$ & $\begin{array}{l}13.5 \\
14.0 \\
13.0 \\
16.5\end{array}$ & $\begin{array}{l}13.5 \\
14.5 \\
15.5 \\
24.5\end{array}$ & $\begin{array}{l}13.5 \\
14.5 \\
15.5 \\
24.0\end{array}$ & $\begin{array}{l}\text { ZIRC } \\
\text { ZIRC } \\
\text { ZIRC } \\
\text { STEEL }\end{array}$ & $\begin{array}{l}\text { ZIRC } \\
\text { ZIRC } \\
\text { ZIRC } \\
\text { STELL }\end{array}$ & $\begin{array}{l}0.0 \\
0.0 \\
0.0 \\
0.0\end{array}$ & $\begin{array}{l}\text { NONE } \\
\text { SAND-B } \\
\text { SAND-B } \\
\text { SAND-B }\end{array}$ \\
\hline D. 1 & $\begin{array}{l}\text { STAB } \\
\text { CAN } \\
\text { SLEEVE }\end{array}$ & $\begin{array}{l}12.0 \\
13.5 \\
14.5\end{array}$ & $\begin{array}{l}12.0 \\
1.40 \\
21.5\end{array}$ & $\begin{array}{l}12.0 \\
14.0 \\
21.5\end{array}$ & $\begin{array}{l}12.0 \\
14.5 \\
41.0\end{array}$ & $\begin{array}{l}13.5 \\
14.5 \\
48.9\end{array}$ & $\begin{array}{l}\text { HEL IUT } \\
\text { STELL } \\
\text { IRON }\end{array}$ & $\begin{array}{l}\text { IEL IUM } \\
\text { STEEL } \\
\text { IRON }\end{array}$ & $\begin{array}{r}0.0 \\
0.0 \\
10.0\end{array}$ & $\begin{array}{l}\text { TONE } \\
\text { SAND-B } \\
\text { SAND-B }\end{array}$ \\
\hline
\end{tabular}


Table 4-4. (Continued)

\begin{tabular}{|c|c|c|c|c|c|c|c|c|c|c|}
\hline CORCEPT & ELERERT & $\begin{array}{c}\text { INS } \\
\text { MATE: } \\
\text { IID }\end{array}$ & $\begin{array}{l}\text { DE } \\
\text { OD } \\
\text { OD }\end{array}$ & $\begin{array}{l}\text { OUTSIDE } \\
\text { RATERIAL } \\
\text { OD }\end{array}$ & $\begin{array}{c}\text { PLLER } \\
\text { OD }\end{array}$ & $\begin{array}{l}\text { GAP } \\
\text { OD }\end{array}$ & $\begin{array}{l}\text { IHSIDE, } \\
\text { RATLIAL }\end{array}$ & $\begin{array}{l}\text { DUTSIDE } \\
\text { MATERIAL: }\end{array}$ & $\begin{array}{l}\text { COATIING } \\
\text { DELAY } \\
\text { (YRS) }\end{array}$ & FILLER \\
\hline D.8 & $\begin{array}{l}\text { STAB } \\
\text { CAN } \\
\text { SLEEVT }\end{array}$ & $\begin{array}{l}18.0 \\
13.5 \\
16.5\end{array}$ & 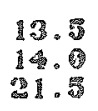 & $\begin{array}{l}13.5 \\
14.0 \\
31.5\end{array}$ & $\begin{array}{l}13.5 \\
14.5 \\
80.0\end{array}$ & $\begin{array}{l}13.5 \\
13.5 \\
40.0\end{array}$ & $\begin{array}{l}\text { STEEL } \\
\text { STERLL } \\
\text { IRON }\end{array}$ & $\begin{array}{l}\text { STEEL } \\
\text { STREL } \\
\text { IROR }\end{array}$ & $\begin{array}{r}0.0 \\
100.0\end{array}$ & $\begin{array}{l}\text { MONE } \\
\text { SARD-R } \\
\text { SAND-R B }\end{array}$ \\
\hline D. 5 & $\begin{array}{l}\text { STPRE } \\
\text { CAN } \\
\text { SLEREE }\end{array}$ & $\begin{array}{l}12.0 \\
13.5 \\
14.5\end{array}$ & $\begin{array}{l}13.5 \\
19.9 \\
16.5\end{array}$ & $\begin{array}{l}13.5 \\
14.9 \\
16.5\end{array}$ & $\begin{array}{l}13.9 \\
14.5 \\
48.0\end{array}$ & $\begin{array}{l}13.5 \\
18.5 \\
410.5\end{array}$ & $\begin{array}{l}\text { STEEL } \\
\text { STERL } \\
\text { ZIRC }\end{array}$ & $\begin{array}{l}\text { STEEL } \\
\text { STREL } \\
\text { ZRR }\end{array}$ & $\begin{array}{r}0.0 \\
100.0\end{array}$ & $\begin{array}{l}\text { NONE } \\
S A N D-B \\
S A N D-B\end{array}$ \\
\hline D1.2 & $\begin{array}{l}\text { STAB } \\
\text { CAN } \\
\text { SLEEVE }\end{array}$ & $\begin{array}{l}12.0 \\
13.8 \\
14.5\end{array}$ & $\begin{array}{l}13.5 \\
14.9 \\
14.9 \\
52.5\end{array}$ & $\begin{array}{l}13.5 \\
13.0 \\
28.5\end{array}$ & $\begin{array}{l}13.5 \\
14.5 \\
4.8 \\
48.5\end{array}$ & $\begin{array}{l}13.5 \\
14.5 \\
40.5\end{array}$ & $\begin{array}{l}\text { 304SST } \\
3045 S T \\
\text { I ROP }\end{array}$ & $\begin{array}{l}\text { 304SST } \\
\text { 304SST } \\
\text { IRON }\end{array}$ & $\begin{array}{r}0.0 \\
0.0 \\
100.0\end{array}$ & $\begin{array}{l}\text { MONE } \\
\text { SAND-B } \\
\text { SAND-B }\end{array}$ \\
\hline D1.5 & $\begin{array}{l}\text { STAB } \\
\text { CAN } \\
\text { GLIEUE }\end{array}$ & $\begin{array}{l}12.0 \\
18.5 \\
14.5\end{array}$ & $\begin{array}{l}13.5 \\
18.5 \\
15.0\end{array}$ & $\begin{array}{l}13.5 \\
14.0 \\
15.0\end{array}$ & $\begin{array}{l}13.5 \\
14.5 \\
2 \% .0\end{array}$ & $\begin{array}{l}13.5 \\
14.5 \\
29.0\end{array}$ & $\begin{array}{l}30458 T \\
30459 \\
304597\end{array}$ & $\begin{array}{l}30459 T \\
30455 T \\
34551\end{array}$ & $\begin{array}{r}0.0 \\
100.0\end{array}$ & $\begin{array}{l}\text { MONE } \\
\text { SAND-B } \\
\text { SAND-D }\end{array}$ \\
\hline D2. $\mathbb{1}$ & $\begin{array}{l}\text { STAB } \\
\text { GAV } \\
\text { OACR } \\
\text { SLEEVE }\end{array}$ & $\begin{array}{l}12.5 \\
13.5 \\
14.5 \\
15.5\end{array}$ & $\begin{array}{l}19.5 \\
14.0 \\
15.9 \\
139.9 \\
23.5\end{array}$ & $\begin{array}{l}13.5 \\
14.0 \\
15.0 \\
83.5\end{array}$ & $\begin{array}{l}13.5 \\
13.8 \\
15.5 \\
48.0 \\
48.0\end{array}$ & $\begin{array}{l}13.5 \\
14.5 \\
15.5 \\
48.5\end{array}$ & $\begin{array}{l}\text { INCONEL } \\
\text { INCONEL } \\
\text { INCONEL } \\
\text { IRON }\end{array}$ & $\begin{array}{l}\text { INCONEL! } \\
\text { INCONEL } \\
\text { IRCONEL } \\
\text { IRON }\end{array}$ & $\begin{array}{r}0.0 \\
0.0 \\
10 \%\end{array}$ & $\begin{array}{l}\text { NONE } \\
\text { SAND-B } \\
\text { SAND-B } \\
\text { SAND-B }\end{array}$ \\
\hline D2.8 & $\begin{array}{l}\text { STAB } \\
\text { GAR } \\
\text { Q PACK } \\
\text { SLEEVE }\end{array}$ & $\begin{array}{l}12.0 \\
13.5 \\
14.5 \\
27.5\end{array}$ & $\begin{array}{l}13.5 \\
14.0 \\
13.0 \\
28.0\end{array}$ & $\begin{array}{l}19.5 \\
19.5 \\
28.0 \\
28.0\end{array}$ & $\begin{array}{l}13.5 \\
14.5 \\
27.5 \\
48.0\end{array}$ & $\begin{array}{l}13.5 \\
14.5 \\
27.5 \\
40.0\end{array}$ & $\begin{array}{l}\text { ZIRC } \\
\text { ZIRC } \\
\text { ZIRC } \\
\text { ZIRC }\end{array}$ & $\begin{array}{l}\text { ZIRC } \\
\text { ZIRC } \\
\text { LEAD } \\
\text { ZIRC }\end{array}$ & $\begin{array}{r}0.0 \\
9.0 \\
10.0\end{array}$ & $\begin{array}{l}\text { PVONE } \\
\text { SAND-B } \\
\text { SARD-B } \\
\text { CLINO }\end{array}$ \\
\hline E. 3 & $\underset{C A M}{C A R B}$ & $\begin{array}{l}12.5 \\
13.5\end{array}$ & $\begin{array}{l}\text { 13. } \\
13.0\end{array}$ & $\begin{array}{l}13.5 \\
11.2\end{array}$ & $\begin{array}{r}13.5 \\
20.76\end{array}$ & $\begin{array}{r}13.5 \\
20.0\end{array}$ & $\begin{array}{l}\text { LEAD } \\
30455 T\end{array}$ & $\begin{array}{l}\text { LEAD } \\
\text { ZIRC }\end{array}$ & 0.0 & $\begin{array}{l}\text { NONE } \\
\text { SAND-B }\end{array}$ \\
\hline 里。象 & $\begin{array}{l}\text { CA STAD } \\
\text { CAN }\end{array}$ & $\begin{array}{l}12.0 \\
13.5\end{array}$ & $\begin{array}{l}13.57 \\
14.0\end{array}$ & $\begin{array}{l}13.5 \\
1 \% .0\end{array}$ & $\begin{array}{l}13.5 \\
20.0\end{array}$ & $\begin{array}{l}13.5 \\
20.0\end{array}$ & $\begin{array}{l}\text { LEAD } \\
\text { ZIRE }\end{array}$ & $\begin{array}{l}\text { LEAD } \\
\text { ZIRC }\end{array}$ & 0.0 & $\begin{array}{l}\text { PONE } \\
\text { SAND-B }\end{array}$ \\
\hline E. 20. & $\begin{array}{l}\text { CAA STAB } \\
\text { GAII }\end{array}$ & $\begin{array}{l}12.6 \\
13.6\end{array}$ & $\frac{13.5}{20.5}$ & $\begin{array}{l}13.8 \\
20.8\end{array}$ & 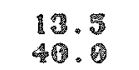 & $\begin{array}{l}13.5 \\
46.5\end{array}$ & $\begin{array}{l}\text { LEAD } \\
30455 T\end{array}$ & $\begin{array}{l}\text { LEAD } \\
\text { ZIRC }\end{array}$ & 9.0 & $\begin{array}{l}\text { ROTE } \\
\text { SARD-B }\end{array}$ \\
\hline
\end{tabular}


Table 4-4. (Continued)

\begin{tabular}{|c|c|c|c|c|c|c|c|c|c|c|}
\hline CONGEPT & DLEMEIT & 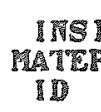 & $\begin{array}{l}\text { DER } \\
\text { OD }\end{array}$ & 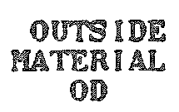 & 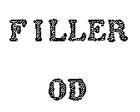 & $\begin{array}{r}\text { GAP } \\
\text { O1 }\end{array}$ & $\begin{array}{l}\text { 1TS 1DEL } \\
\text { MATERIAL }\end{array}$ & $\begin{array}{l}\text { OUTS IDE } \\
\text { HATRR }\end{array}$ & $\begin{array}{l}\text { COATIVG } \\
\text { DELAY } \\
\text { TIRS }\end{array}$ & TLERE \\
\hline 琶。1 & $\begin{array}{l}\text { CA } \\
\text { GAPA }\end{array}$ & $\begin{array}{l}12.0 \\
13.5\end{array}$ & 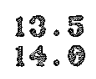 & $\begin{array}{l}13.5 \\
16.0\end{array}$ & $\begin{array}{l}13.5 \\
48.0 \\
460\end{array}$ & $\begin{array}{r}13.5 \\
48.0\end{array}$ & $\begin{array}{l}\text { LEAD } \\
\text { STEREL }\end{array}$ & $\begin{array}{l}\text { LEAD } \\
\text { STEEL }\end{array}$ & 0.0 & $\begin{array}{l}\text { NONE } \\
\text { SAID-B }\end{array}$ \\
\hline 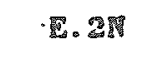 & $\begin{array}{l}\text { CA STAD } \\
\text { CAM }\end{array}$ & $\begin{array}{l}12.0 \\
13.5\end{array}$ & $\begin{array}{l}13.5 \\
14.0\end{array}$ & $\begin{array}{l}13.5 \\
11.0\end{array}$ & $\begin{array}{l}13.5 \\
48.06\end{array}$ & $\begin{array}{l}13.5 \\
48.0\end{array}$ & 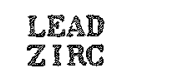 & $\frac{\text { LEAD }}{\text { ZIMC }}$ & 0.0 & $\begin{array}{l}\text { NONE } \\
\text { SAND-B }\end{array}$ \\
\hline 宦。3 & $\begin{array}{l}\text { CA STAR } \\
\text { CAM }\end{array}$ & $\begin{array}{l}12.0 \\
15.53\end{array}$ & $\begin{array}{l}13.5 \\
13.5\end{array}$ & $\begin{array}{l}13.5 \\
13.5\end{array}$ & $\begin{array}{r}13.5 \\
48.0\end{array}$ & $\begin{array}{l}13.5 \\
48.5\end{array}$ & $\begin{array}{l}\text { LEAO } \\
\text { MCONEL }\end{array}$ & $\begin{array}{l}\text { LEAD } \\
\text { IRCONELI }\end{array}$ & 9.0 & $\begin{array}{l}\text { NOHE } \\
\text { S.NND }-B\end{array}$ \\
\hline E. 4 . & $\begin{array}{l}\text { CA STAB } \\
\text { CAN }\end{array}$ & $\begin{array}{l}12.0 \\
13.5\end{array}$ & $\begin{array}{l}10.5 \\
10.0\end{array}$ & $\begin{array}{l}13.5 \\
13.0\end{array}$ & $\begin{array}{l}13.5 \\
48.0\end{array}$ & $\begin{array}{l}13.3 \\
89.0\end{array}$ & $\begin{array}{l}\text { LEAD } \\
3045 S T\end{array}$ & $\begin{array}{l}\text { LEAD } \\
30455 T\end{array}$ & 0.0 & $\begin{array}{l}\text { NOPEE } \\
\text { SAND-B }\end{array}$ \\
\hline 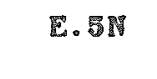 & $\begin{array}{l}\text { GA STA } \\
\text { GAM }\end{array}$ & $\begin{array}{l}12.0 \\
13.5\end{array}$ & $\begin{array}{l}13.5 \\
14.96\end{array}$ & $\begin{array}{l}13.5 \\
14.0\end{array}$ & 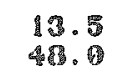 & $\begin{array}{l}13.5 \\
48.9\end{array}$ & $\begin{array}{l}\text { LFAD } \\
\text { COPPRR. }\end{array}$ & $\begin{array}{l}\text { LEAD } \\
\text { COPETER }\end{array}$ & . & $\begin{array}{l}\text { NOPIE } \\
\text { SAND-D }\end{array}$ \\
\hline 2.6n & $\begin{array}{l}\text { CA STAB } \\
\text { CAN }\end{array}$ & $\begin{array}{l}12.0 \\
10.0\end{array}$ & $\begin{array}{l}13.5 \\
13.09\end{array}$ & $\begin{array}{l}13.5 \\
1.50\end{array}$ & $\begin{array}{l}13.5 \\
43.0\end{array}$ & $\begin{array}{l}13.5 \\
48.5\end{array}$ & $\begin{array}{l}\text { LEADD } \\
\text { LFAD }\end{array}$ & $\begin{array}{l}\text { LEAD } \\
\text { LEAD }\end{array}$ & 9.0 & $\begin{array}{l}\text { NONE } \\
\text { SAND-B }\end{array}$ \\
\hline 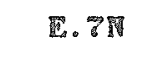 & $\begin{array}{l}\text { CA } 5 \text { TAD } \\
\text { CAM }\end{array}$ & $\begin{array}{l}12.0 \\
13.9\end{array}$ & $\begin{array}{l}13.5 \\
14.0\end{array}$ & $\begin{array}{l}13.9 \\
14.9\end{array}$ & $\begin{array}{l}13.5 \\
48.0\end{array}$ & $\begin{array}{l}13.5 \\
4.6 \%\end{array}$ & $\begin{array}{l}\text { LEAD } \\
\text { IRON }\end{array}$ & $\begin{array}{l}\text { LEAT } \\
\text { MONON }\end{array}$ & $\begin{array}{l}0.0 \\
0.0\end{array}$ & $\begin{array}{l}\text { NOPUP } \\
\text { SAND-B }\end{array}$ \\
\hline T.8In & $\begin{array}{l}\text { CA STAB } \\
\text { CAM }\end{array}$ & $\begin{array}{l}12.0 \\
13.5\end{array}$ & $\begin{array}{l}13.5 \\
19.5\end{array}$ & $\begin{array}{l}13.5 \\
19.5\end{array}$ & $\begin{array}{l}12.78 \\
48.0\end{array}$ & $\begin{array}{l}13.5 \\
48.6\end{array}$ & $\begin{array}{l}\text { LEAD } \\
\text { STEEL }\end{array}$ & $\begin{array}{l}\text { LEAD } \\
\text { STELL }\end{array}$ & $\begin{array}{l}0.10 \\
0.0\end{array}$ & $\begin{array}{l}\text { NONR } \\
\text { SAND-B }\end{array}$ \\
\hline 蛋:919 & $\begin{array}{l}\text { CA STAB } \\
\text { CAI }\end{array}$ & $\begin{array}{l}12.5 \\
13.5\end{array}$ & $\begin{array}{l}13.3 \\
19.5\end{array}$ & $\begin{array}{l}13.5 \\
19.5\end{array}$ & $\begin{array}{l}13.5 \\
48.0\end{array}$ & $\begin{array}{l}13.5 \\
48.0\end{array}$ & $\begin{array}{l}\text { LEAD } \\
\text { ZIRC }\end{array}$ & $\begin{array}{l}\text { LEAD } \\
\mathbb{Z I R C}\end{array}$ & 0.9 & $\begin{array}{l}\text { MONE } \\
\text { SAND-B }\end{array}$ \\
\hline E. 101 & $\begin{array}{l}\text { CA STAB } \\
\text { CAN }\end{array}$ & $\begin{array}{l}13.6 \\
13.5\end{array}$ & $\begin{array}{l}19.5 \\
19.58\end{array}$ & $\begin{array}{l}13.5 \\
19.5\end{array}$ & $\begin{array}{l}13.5 \\
40.0\end{array}$ & $\begin{array}{l}13.5 \\
48.0\end{array}$ & $\begin{array}{l}\text { LEAD } \\
\text { INCONRED }\end{array}$ & $\begin{array}{l}\text { LEAD } \\
\text { INCONTL }\end{array}$ & $\begin{array}{r}0 \\
0.0\end{array}$ & $\begin{array}{l}\text { NONE } \\
\text { SAND-B }\end{array}$ \\
\hline 要。11 & $\begin{array}{l}\text { CA } S T A B \\
\text { CAM }\end{array}$ & $\begin{array}{l}12.0 \\
13.5\end{array}$ & $\begin{array}{l}13.5 \\
19.5\end{array}$ & $\begin{array}{l}13.8 \\
19.8\end{array}$ & $\begin{array}{l}13.5 \\
45.7\end{array}$ & $\begin{array}{l}13.5 \\
48.5\end{array}$ & $\begin{array}{l}\text { LEAD } \\
35459 T\end{array}$ & $\begin{array}{l}\text { LEAD } \\
\text { 304SET }\end{array}$ & 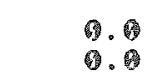 & $\begin{array}{l}\text { NONE } \\
\text { SARD-B }\end{array}$ \\
\hline
\end{tabular}


Table 4-4. (Continued)

\begin{tabular}{|c|c|c|c|c|c|c|c|c|c|c|}
\hline COMCEPT & ELEMENT & $\begin{array}{c}\text { INSI } \\
\text { MATER } \\
\text { ID }\end{array}$ & $\begin{array}{l}\text { IDE } \\
\text { IAL } \\
\text { OD }\end{array}$ & $\begin{array}{l}\text { OUTSIDE } \\
\text { MATERIAL } \\
\text { OD }\end{array}$ & $\begin{array}{c}\text { FIULR } \\
\text { OD }\end{array}$ & $\begin{array}{r}\text { GAP } \\
\text { OD }\end{array}$ & $\begin{array}{l}\text { IMSIDE } \\
\text { MATERIAL }\end{array}$ & $\begin{array}{l}\text { OUTSIDE: } \\
\text { MATERIAL| }\end{array}$ & $\begin{array}{l}\text { COATING } \\
\text { DELAYY } \\
\text { (YRS) }\end{array}$ & FILLER \\
\hline E. $12 \mathrm{~N}$ & $\begin{array}{l}\text { CA STAB } \\
\text { CAIN }\end{array}$ & $\begin{array}{l}12.0 \\
13.5\end{array}$ & $\begin{array}{l}13.5 \\
19.5\end{array}$ & $\begin{array}{l}19.5 \\
19.5\end{array}$ & $\begin{array}{l}13.5 \\
48.0\end{array}$ & $\begin{array}{l}13.5 \\
48.0\end{array}$ & $\begin{array}{l}\text { LEAD } \\
\text { COPPER. }\end{array}$ & $\begin{array}{l}\text { LEAD } \\
\text { COPPER }\end{array}$ & 0.0 & $\begin{array}{l}\text { NONE } \\
\text { SAND }-B\end{array}$ \\
\hline E. $13 \mathrm{~N}$ & $\begin{array}{l}\text { CA } \$ T A B \\
\text { CAN }\end{array}$ & $\begin{array}{l}12.0 \\
13.8\end{array}$ & $\begin{array}{l}13.5 \\
19.5\end{array}$ & $\begin{array}{l}13.5 \\
19.5\end{array}$ & $\begin{array}{l}13.5 \\
48.0\end{array}$ & $\begin{array}{l}13.5 \\
48.1\end{array}$ & $\begin{array}{l}\text { LEAD } \\
\text { LEAD }\end{array}$ & $\begin{array}{l}\mathrm{LEAD} \\
\mathrm{LEAD}\end{array}$ & 0.0 & $\begin{array}{l}\text { NONE } \\
\text { SAND-B }\end{array}$ \\
\hline E. $14 \mathrm{~N}$ & $\begin{array}{l}\text { CA STAB } \\
\text { CAN }\end{array}$ & $\begin{array}{l}12.5 \\
13.5\end{array}$ & $\begin{array}{l}13.5 \\
19.5\end{array}$ & $\begin{array}{l}13.5 \\
19.8\end{array}$ & $\begin{array}{l}13.5 \\
48.0\end{array}$ & $\begin{array}{l}13.5 \\
48.0\end{array}$ & $\begin{array}{l}\text { LEAD } \\
\text { IRON }\end{array}$ & $\begin{array}{l}\text { LEAD } \\
\text { IRON }\end{array}$ & 0.0 & $\begin{array}{l}\text { NONE } \\
\text { SAMD-B }\end{array}$ \\
\hline E. $15 \mathrm{~N}$ & $\begin{array}{l}\text { CA STAB } \\
\text { CAN }\end{array}$ & $\begin{array}{l}12.0 \\
13.5\end{array}$ & $\begin{array}{l}13.5 \\
14.0\end{array}$ & 13.5 & $\begin{array}{l}13.5 \\
36.0\end{array}$ & $\begin{array}{l}13.5 \\
36.0\end{array}$ & $\begin{array}{l}\text { LEAD } \\
\text { ZIRC }\end{array}$ & $\begin{array}{l}\text { LEAD } \\
\text { ZIMC }\end{array}$ & 0.0 & $\begin{array}{l}\text { NONE } \\
\text { SAND-B }\end{array}$ \\
\hline E. $16 \mathrm{~N}$ & $\begin{array}{l}\text { CA STAB } \\
\text { CAN }\end{array}$ & $\begin{array}{l}13.0 \\
13.5\end{array}$ & $\begin{array}{l}13.5 \\
14.0\end{array}$ & $\begin{array}{l}13.5 \\
14.0\end{array}$ & $\begin{array}{l}13.5 \\
36.0\end{array}$ & $\begin{array}{l}13.5 \\
36.0\end{array}$ & $\begin{array}{l}\text { LEAD } \\
\text { INCONEL }\end{array}$ & $\begin{array}{l}\text { LEAD } \\
\text { INCONEL: }\end{array}$ & 0 & $\begin{array}{l}\text { NOREE } \\
\text { SAND - B }\end{array}$ \\
\hline E. 17\% & $\begin{array}{l}\text { CA STAB } \\
\text { CAN }\end{array}$ & $\begin{array}{l}12.0 \\
13.5\end{array}$ & $\begin{array}{l}13.5 \\
14.0\end{array}$ & $\begin{array}{l}13.5 \\
14.0\end{array}$ & $\begin{array}{l}13.5 \\
20.0\end{array}$ & $\begin{array}{l}13.5 \\
20.6\end{array}$ & $\begin{array}{l}\text { LEAD } \\
\text { ZIRC }\end{array}$ & $\begin{array}{l}\text { LEAD } \\
\text { ZIRC }\end{array}$ & 0.0 & $\begin{array}{l}\text { NORE } \\
\text { SARD-B }\end{array}$ \\
\hline E. 188 & $\begin{array}{l}\text { CA STAB } \\
\text { CAN }\end{array}$ & $\begin{array}{l}12.0 \\
13.5\end{array}$ & $\begin{array}{l}13.5 \\
14.0\end{array}$ & $\begin{array}{l}13.5 \\
14.0\end{array}$ & $\begin{array}{l}13.5 \\
20.0\end{array}$ & $\begin{array}{l}13.5 \\
20.0\end{array}$ & $\begin{array}{l}\text { LEAD } \\
\text { INCONEL }\end{array}$ & $\begin{array}{l}\text { LEAD } \\
\text { INCONEL: }\end{array}$ & 0.0 & $\begin{array}{l}\text { NONE } \\
\text { SAND-B }\end{array}$ \\
\hline E. $19 \mathrm{~N}$ & $\begin{array}{l}\text { CA STAB } \\
\text { CAN }\end{array}$ & $\begin{array}{l}12.0 \\
13.5\end{array}$ & $\begin{array}{l}13.5 \\
14.9\end{array}$ & $\begin{array}{l}13.5 \\
14.0\end{array}$ & $\begin{array}{l}13.5 \\
48.8\end{array}$ & $\begin{array}{r}13.5 \\
\$ 8.0\end{array}$ & $\begin{array}{l}\text { LEAD } \\
\text { ZIRC }\end{array}$ & $\begin{array}{l}\text { LEAD } \\
\text { ZIRC }\end{array}$ & 0.0 & $\begin{array}{l}\text { NONE } \\
\text { BENT }\end{array}$ \\
\hline E.20N & $\begin{array}{l}\text { CA STAB } \\
\text { CAN }\end{array}$ & $\begin{array}{l}12.0 \\
13.5\end{array}$ & $\begin{array}{l}13.5 \\
14.0\end{array}$ & $\begin{array}{l}13.5 \\
14.0\end{array}$ & $\begin{array}{r}13.5 \\
48.0\end{array}$ & $\begin{array}{l}13.5 \\
48.0\end{array}$ & $\begin{array}{l}\text { LEAD } \\
\text { IRCONEL }\end{array}$ & $\begin{array}{l}\text { LEAD } \\
\text { INCONEL }\end{array}$ & $\begin{array}{l}9.0 \\
9.0\end{array}$ & $\begin{array}{l}\text { PONE } \\
\text { BENT }\end{array}$ \\
\hline E.P1N & $\begin{array}{l}\text { CA STAB } \\
\text { CAN }\end{array}$ & $\begin{array}{l}12.0 \\
13.5\end{array}$ & $\begin{array}{l}13.5 \\
14.0\end{array}$ & $\begin{array}{l}13.5 \\
14.8\end{array}$ & $\begin{array}{l}13.5 \\
48.0\end{array}$ & $\begin{array}{r}13.5 \\
48.0\end{array}$ & $\frac{\operatorname{LEAD}}{\mathrm{ZIRC}}$ & $\begin{array}{l}\text { LEAD } \\
\text { ZIRC }\end{array}$ & 0.0 & $\begin{array}{l}\text { NONE } \\
\text { CLINO }\end{array}$ \\
\hline E. $22 \pi$ & $\begin{array}{l}\text { CA STAB } \\
\text { CAN }\end{array}$ & $\begin{array}{l}12.5 \\
13.5\end{array}$ & $\begin{array}{l}13.5 \\
14.9\end{array}$ & $\begin{array}{l}13.5 \\
14.0\end{array}$ & $\begin{array}{l}13.5 \\
48.9\end{array}$ & $\begin{array}{l}13.5 \\
43.0\end{array}$ & $\begin{array}{l}\text { LEAD } \\
\text { INCONEL }\end{array}$ & $\begin{array}{l}\text { LEAD } \\
\text { INCONEL, }\end{array}$ & 9.0 & $\begin{array}{l}\text { MONE } \\
\text { CLINO }\end{array}$ \\
\hline
\end{tabular}


Table 4-4. (Continued)

\begin{tabular}{|c|c|c|c|c|c|c|c|c|c|c|}
\hline CONCEPT & ELENCWT & MATEI & $\begin{array}{l}\text { IDF } \\
\text { OD } \\
\text { OD }\end{array}$ & $\begin{array}{l}\text { OUTS IDE } \\
\text { PATERIAL } \\
\text { OD }\end{array}$ & $\begin{array}{c}\text { FLLER } \\
\text { OD }\end{array}$ & $\begin{array}{l}\text { GAP } \\
\text { OD }\end{array}$ & $\begin{array}{l}\text { INS IDEI } \\
\text { MATERIAL }\end{array}$ & $\begin{array}{l}\text { OUTS IDE } \\
\text { MATERIAL: }\end{array}$ & $\begin{array}{l}\text { COATING } \\
\text { DELAY } \\
\text { (YRS) }\end{array}$ & FILLER \\
\hline E. $23 N$ & $\begin{array}{l}\text { CA STAB } \\
\text { CAN }\end{array}$ & $\begin{array}{l}12.0 \\
13.5\end{array}$ & $\begin{array}{l}13.5 \\
14.0\end{array}$ & $\begin{array}{l}13.5 \\
14.0\end{array}$ & $\begin{array}{r}13.5 \\
48.0\end{array}$ & $\begin{array}{l}13.5 \\
48.0\end{array}$ & $\begin{array}{l}\text { LEAD } \\
\text { STELL }\end{array}$ & $\begin{array}{l}\text { LEAD } \\
\text { STEEL }\end{array}$ & 0.0 & $\begin{array}{l}\text { PONE } \\
\text { SAND-B }\end{array}$ \\
\hline E. $24 N$ & $\begin{array}{l}\text { CA STAB } \\
\text { CAN }\end{array}$ & $\begin{array}{l}12.0 \\
13.5\end{array}$ & $\begin{array}{l}13.5 \\
13.0\end{array}$ & $\begin{array}{l}13.5 \\
14.0\end{array}$ & $\begin{array}{l}13.5 \\
48.0\end{array}$ & $\begin{array}{l}13.5 \\
48.0\end{array}$ & $\begin{array}{l}\text { LEAD } \\
\text { ZIRC }\end{array}$ & $\begin{array}{l}\text { LEAD } \\
\text { ZIRC }\end{array}$ & 0.0 & $\begin{array}{l}\text { HONE } \\
\text { SAND-B }\end{array}$ \\
\hline E. 2517 & $\begin{array}{l}\text { CA STAE } \\
\text { CAN }\end{array}$ & $\begin{array}{l}12.0 \\
13.5\end{array}$ & $\begin{array}{l}13.5 \\
14.0\end{array}$ & $\begin{array}{l}13.5 \\
14.0\end{array}$ & $\begin{array}{l}13.5 \\
48.0\end{array}$ & $\begin{array}{l}13.5 \\
48.0\end{array}$ & $\begin{array}{l}\text { LEAD } \\
\text { INCONEL }\end{array}$ & $\begin{array}{l}\text { LEAD } \\
\text { INCONEL. }\end{array}$ & 0.0 & $\begin{array}{l}\text { NONE } \\
\text { SAND-B }\end{array}$ \\
\hline E. 267 & $\begin{array}{l}\text { CA STAB } \\
\text { CAR }\end{array}$ & $\begin{array}{l}12.0 \\
13.5\end{array}$ & $\begin{array}{l}13.5 \\
14.0\end{array}$ & $\begin{array}{l}13.5 \\
14.0\end{array}$ & $\begin{array}{l}13.5 \\
48.0\end{array}$ & $\begin{array}{l}13.5 \\
48.0\end{array}$ & $\begin{array}{l}\text { LEAD } \\
304 S S T\end{array}$ & $\begin{array}{l}\text { LEAD } \\
304 S S T\end{array}$ & 9.0 & $\begin{array}{l}\text { PONE } \\
\text { SAND-B }\end{array}$ \\
\hline E. $27 \mathbb{N}$ & $\begin{array}{l}\text { CA STAE } \\
\text { CAN }\end{array}$ & $\begin{array}{l}12.0 \\
13.3\end{array}$ & $\begin{array}{l}13.5 \\
14.0\end{array}$ & $\begin{array}{l}13.5 \\
14.0\end{array}$ & $\begin{array}{l}13.5 \\
48.0\end{array}$ & $\begin{array}{l}13.5 \\
48.9\end{array}$ & $\begin{array}{l}\text { LEAD } \\
\text { COPPER. }\end{array}$ & $\begin{array}{l}\text { LEAD } \\
\text { COPPER }\end{array}$ & 0.0 & $\begin{array}{l}\text { NONE } \\
\text { SAND-B }\end{array}$ \\
\hline R. $2 B N$ & $\begin{array}{l}\text { CA STAB } \\
\text { CAM }\end{array}$ & $\begin{array}{l}12.0 \\
13.5\end{array}$ & $\begin{array}{l}13.5 \\
1.50\end{array}$ & $\begin{array}{l}13.5 \\
14.0\end{array}$ & $\begin{array}{l}13.5 \\
48.0\end{array}$ & $\begin{array}{l}13.5 \\
48.0\end{array}$ & $\begin{array}{l}\text { LEAD } \\
\text { LEAD }\end{array}$ & $\begin{array}{l}\text { LEAD } \\
\text { LEAD }\end{array}$ & 0.0 & $\begin{array}{l}\text { TONE } \\
\text { SAND-B }\end{array}$ \\
\hline E. $29 \%$ & $\begin{array}{l}\text { CA STAB } \\
\text { CAN }\end{array}$ & $\begin{array}{l}12.0 \\
13.5\end{array}$ & $\begin{array}{l}13.5 \\
1.0\end{array}$ & $\begin{array}{l}13.5 \\
14.0\end{array}$ & $\begin{array}{l}13.5 \\
417.0\end{array}$ & $\begin{array}{l}13.5 \\
48.0\end{array}$ & $\begin{array}{l}\text { LFAD } \\
\text { InON }\end{array}$ & $\begin{array}{l}\text { LEAD } \\
\text { IRON }\end{array}$ & 9.9 & $\begin{array}{l}\text { NONE } \\
\text { SAND - B }\end{array}$ \\
\hline E.30N & $\begin{array}{l}\text { CA STAB } \\
\text { CAR }\end{array}$ & 12.0 & $\begin{array}{l}13.5 \\
1.40\end{array}$ & $\begin{array}{l}13.5 \\
14.1\end{array}$ & $\begin{array}{l}13.5 \\
48.0\end{array}$ & $\begin{array}{r}13.5 \\
48.0\end{array}$ & $\frac{\text { LEAD }}{\text { STELL }}$ & $\begin{array}{l}\text { LEAD } \\
\text { ZIRC }\end{array}$ & 0.0 & $\begin{array}{l}\text { NONE } \\
\text { SAND-B }\end{array}$ \\
\hline E. 311 & $\begin{array}{l}\text { CA STAD } \\
\text { CAN }\end{array}$ & $\begin{array}{l}12.0 \\
13.5\end{array}$ & $\begin{array}{l}13.5 \\
14.0\end{array}$ & $\begin{array}{l}13.5 \\
14.5\end{array}$ & $\begin{array}{l}13.5 \\
48.5\end{array}$ & $\begin{array}{l}13.5 \\
40.0\end{array}$ & $\begin{array}{l}\text { LEAD } \\
\text { STEEL }\end{array}$ & $\begin{array}{l}\text { LEAD } \\
\text { ZIRC }\end{array}$ & 0.0 & $\begin{array}{l}\text { NONE } \\
\text { SAND-B }\end{array}$ \\
\hline
\end{tabular}


126 


\section{RESULTS}

\section{1 "BEST" PACKAGES FROM PREVIOUS WORK}

In previous barrier performance studies (Lester, 1979) (Stula, 1980a), various waste package designs were evaluated in four geologic media: salt, shale, basalt, and granite. In each package design category, the case resulting in the longest leach begin time was considered to be the "best" design case of that category. For comparison, performance of these "best" case designs was evaluated with the current version of the BARIER code. This comparison is presented in Table 5-1 for salt, shale and basalt geologies. A more detailed summary of calculations for these cases in the current study is presented in Table 5-2. The current model generally predicts leach begin times in basalt which are lower than in previous results. Current results compared to previous results in salt and shale give lower leach begin times for long-lived packages and higher leach begin times for relatively short-lived packages.

For completeness, Table 5-3 presents the best package designs in the current study for each geology. However, it should be noted that not every package design was evaluated in all of the geologies considered.

\subsection{CAST STABILIZER CONCEPT (Concept E)}

On the basis of previous work, a package design utilizing a solid cast stabilizer (Concept E) appeared to be one of the more promising package design candidates. As a result, a large part of the current study deals with Concept $\Xi$ and its design variations.

Results of calculations for the Concept E package design variations are tabulated in Table 5-4. Calculations were perfomed primarily in creeping geologic media with most of the cases evaluated in salt. Comparison of package designs varying only in canister material shows a large variance in leach begin time. Along with a significant dependence on canister thickness (E.1id - E.14iv), this indicates that corrosion resistance is the life determining factor for the concept E design. The use of different backfill materials and variable backfill 
Table 5-1. Comparison of Previous Best Package Designs with Current Results.

\begin{tabular}{|c|c|c|c|c|c|}
\hline \multirow[b]{2}{*}{ Geology } & \multirow{2}{*}{$\begin{array}{l}\text { Package } \\
\text { Design }\end{array}$} & \multicolumn{2}{|c|}{ Leach Begin Time, (yrs) } & \multirow{2}{*}{$\begin{array}{l}\text { Release Begin Time } \\
\text { for Plutonium, (yrs) } \\
\text { (Oxic Conditions) }\end{array}$} & \multirow{2}{*}{$\begin{array}{l}\text { Release End Time } \\
\text { for Plutonium, (yrs) } \\
\text { (0xic Conditions) }\end{array}$} \\
\hline & & Previous & Current & & \\
\hline \multirow[t]{9}{*}{ Salt } & A. 1 & 14 & 1 & $2.8 \times 10^{4}$ & $2.8 \times 10^{5}$ \\
\hline & A. 5 & 14 & 5 & 5 & $4.0 \times 10^{5}$ \\
\hline & B1.11 & 1,900 & 1,000 & $2.9 \times 10^{4}$ & $2.8 \times 10^{5}$ \\
\hline & $\mathrm{Cl} .1$ & 13 & 20 & $2.8 \times 10^{4}$ & $2.8 \times 10^{5}$ \\
\hline & $\mathrm{Cl} .3$ & 13 & 20 & $2.8 \times 10^{4}$ & $2.8 \times 10^{5}$ \\
\hline & 0.3 & 120 & 110 & $2.8 \times 10^{4}$ & $2.8 \times 10^{5}$ \\
\hline & 01.2 & 130 & 110 & $2.8 \times 10^{4}$ & $2.8 \times 10^{5}$ \\
\hline & D2.1 & 120 & 120 & $2.8 \times 10^{4}$ & $2.8 \times 10^{5}$ \\
\hline & E.4 & 6,300 & 2,500 & 2,500 & $3.9 \times 10^{5}$ \\
\hline \multirow[t]{9}{*}{ Shale } & A. 1 & 30 & 1 & $2.8 \times 10^{4}$ & $2.8 \times 10^{5}$ \\
\hline & A. 5 & 30 & 820 & 826 & $4.0 \times 10^{5}$ \\
\hline & B1.11 & 1.900 & 1,100 & $2.9 \times 10^{4}$ & $2.8 \times 10^{5}$ \\
\hline & 01.1 & 27 & 80 & $2.8 \times 10^{4}$ & $2.8 \times 10^{5}$ \\
\hline & $C 1.3$ & 27 & 80 & $2.8 \times 10^{4}$ & $2.8 \times 10^{5}$ \\
\hline & 0.1 & 37 & 80 & $2.8 \times 10^{4}$ & $2.8 \times 10^{5}$ \\
\hline & 01.2 & 140 & 990 & $2.8 \times 10^{4}$ & $2.8 \times 10^{5}$ \\
\hline & 02.1 & 140 & 200 & $2.8 \times 10^{4}$ & $2.8 \times 10^{5}$ \\
\hline & E.24 & 14,000 & 13,000 & $4.1 \times 10^{4}$ & $2.9 \times 10^{5}$ \\
\hline \multirow[t]{8}{*}{ Basalt } & A. 10 & 25,000 & 10,000 & $2.9 \times 10^{4}$ & $2.9 \times 10^{5}$ \\
\hline & B. 8 & 25,000 & 10,000 & $1.0 \times 10^{4}$ & $4.2 \times 10^{5}$ \\
\hline & BI. 7 & 810 & .000 & 4,000 & $2.7 \times 10^{5}$ \\
\hline & 0.7 & 49,000 & 20,000 & $4.6 \times 10^{4}$ & $2.9 \times 10^{5}$ \\
\hline & 01.6 & 12,000 & 4,900 & 4.900 & $3.5 \times 10^{5}$ \\
\hline & 0.5 & 25,000 & 10,000 & $3.8 \times 10^{4}$ & $2.9 \times 10^{5}$ \\
\hline & D1.5 & 1.700 & 1.800 & 1.800 & $4.1 \times 10^{5}$ \\
\hline & 02.8 & 19,000 & 13.000 & $4.1 \times 10^{4}$ & $2.9 \times 10^{5}$ \\
\hline
\end{tabular}


Table 5-2. Previous Best* Package Design Results with Current BARIER Model.

\begin{tabular}{|c|c|c|c|c|c|c|c|c|c|c|c|c|c|}
\hline $\begin{array}{l}\text { packaGe } \\
\text { possigN }\end{array}$ & MEOSA & $\begin{array}{c}\text { SYABUIIIFR } \\
\text { MARERIAL }\end{array}$ & $\begin{array}{l}\text { CAMHSTER } \\
\text { MATERLML }\end{array}$ & 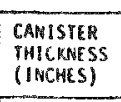 & $\begin{array}{c}\text { SLEFVE } \\
\text { MATERIAL } \\
\end{array}$ & 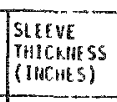 & $\begin{array}{l}\text { BachriL } \\
\text { MAIrRRALL }\end{array}$ & 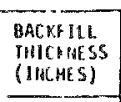 & 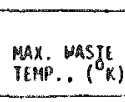 & ALACH BLG & IIIME (rNS) & 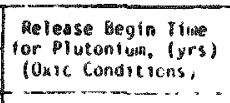 & 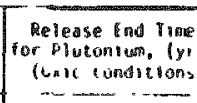 \\
\hline A.1 & sale & 3004St & 304SST & 0.25 & $\cdots$ & $\cdots$ & Sand-8 & 13.0 & 579 & 1 & 1 & $2.8 \times 10^{8}$ & $2.8 \times 10^{5}$ \\
\hline 4.5 & & $3065 s T$ & $30455 T$ (1. $0^{*}$ Thick) & 8.0 & $\ldots$ & $\cdots$ & $\operatorname{sands} \theta$ & 2.25 & $\$ 94$ & 100 & 5 & s & $4.0 \times 10^{5}$ \\
\hline A. 10 & & zirceloy & Zirealoy $\left(1.0^{\prime \prime}\right.$ Thick & 1.0 & $\cdots$ & $\cdots$ & Sand-B & 2.25 & 894 & 5.100 & 5.100 & 5,300 & $4.0 \times 10^{5}$ \\
\hline 8.8 & & Steel & Stoes & 0.25 & $\begin{array}{l}\text { 2ircaloy } \\
\text { (overpack) }\end{array}$ & 1.0 & Sand-B & 1.75 & 491 & 4,800 & 4,200 & 4.800 & $0.1 \times 10^{5}$ \\
\hline 81.8 & & seee & Steel & 0.25 & $\begin{array}{l}\text { Stee! } \\
\text { Lead) }\end{array}$ & 0.25 & clifno & 3.5 & 520 & 2 & 2 & 2 & $2.6 \times 10^{5}$ \\
\hline 81.11 & & steel & stee? & 0.25 & $\begin{array}{l}\operatorname{Iron}(+0 .\}^{n} \\
\text { IIrcal oy })^{n}\end{array}$ & 4.0 & $\operatorname{Sand-B}$ & 12.65 & 551 & 1.100 & 1,01000 & $2.9 \times 10^{8}$ & $2.8 \times 10^{5}$ \\
\hline c. & & neitiven & zircealay & 8.0 & $\begin{array}{l}\text { Zircaloy } \\
\text { (overpack) }\end{array}$ & 1.8 & Send-B & 11.0 & 558 & 9,100 & 9.100 & $3.6 \times 10^{8}$ & $2.8 \times 10^{5}$ \\
\hline c.1 & & Inconel & Inconel & 0.25 & $\begin{array}{l}\text { Iron (+o. } 25 \% \\
\text { 304sst aver- } \\
\text { pack) }\end{array}$ & 3.5 & Sand-B & 11.25 & 549 & 65 & 20 & $2.8 \times 10^{6}$ & $2.8 \times 10^{5}$ \\
\hline $\mathrm{cl} .3$ & & nellium & inconel & 0.25 & $\begin{array}{l}\text { Iron (+0. } 255^{4} \\
3045 s\} \text { over- } \\
\text { pack) }\end{array}$ & 3.5 & Sand-B & 11.25 & 551 & 65 & 20 & $2.8 \times 10^{4}$ & $2.8 \times 10^{5}$ \\
\hline c1.6 & & zircaloy & zircaloy & 0.25 & $\begin{array}{l}\text { Steel }(+0.25 " \\
\text { Zircaloy } \\
\text { overpack) }\end{array}$ & 0.5 & Sand-B & 3.75 & 514 & 30 & 30 & 30 & $3.4 \times 10^{5}$ \\
\hline 0.1 & & 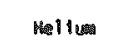 & stoel & 0.25 & Iron & 3.5 & Sond-8 & 13.25 & 559 & 65 & 19 & $2.8 \times 10^{4}$ & $2.8 \times 10^{5}$ \\
\hline 0.3 & & Steel & Steel & 0.25 & Iron & 3.5 & Sand-B & 13.25 & 557 & 160 & 110 & $2.8 \times 10^{8}$ & $2.8 \times 10^{5}$ \\
\hline 0.5 & & steel & steel & 0.25 & zircaloy & 1.0 & Sand-B & 15.75 & 585 & 9.400 & 4,400 & $3.2 \times 10^{4}$ & $2.8 \times 10^{5}$ \\
\hline 01.2 & & 3045ST & 3005ST & 0.25 & Iran & 4.0 & Sand-8 & 12.75 & 552 & 170 & 110 & $2.8 \times 10^{8}$ & $2.8 \times 10^{5}$ \\
\hline 01.5 & & $3045 s r$ & 3045ST & 0.25 & 3045ST & 0.25 & Sand-B & 2.5 & 501 & 2 & 2 & 2 & $4.0 \times 10^{5}$ \\
\hline 02.1 & & Incenel & Incenel & 0.25 & $\begin{array}{l}\text { Irom (*0.25* } \\
\text { Inconel over- } \\
\text { pack) }\end{array}$ & 4.0 & Sand-8 & 12.25 & 551 & 180 & 120 & $2.8 \times 10^{4}$ & $2.8 \times 10^{5}$ \\
\hline 02.8 & & zircatoy & Zircelay & 0.25 & 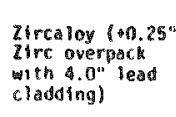 & 0.25 & clino & 10.0 & 574 & 30 & 30 & $2.8 \times 10^{8}$ & $2.8 \times 10^{5}$ \\
\hline E.3 & & Leed & $30455 \mathrm{~T}(+0.8 \mathrm{z} \mathrm{z} \mathrm{rC})$ & 0.25 & $\cdots$ & --- & Sand-8 & 2.9 & 503 & 3.700 & 1,000 & 1,0000 & $3.9 \times 10^{5}$ \\
\hline 8.4 & & Leas & incralay & 0.25 & $\cdots$ & $\cdots$ & $\operatorname{sind}-B$ & 3.0 & 505 & 2.600 & 2.500 & 2.500 & $3.9 \times 10^{5}$ \\
\hline$\varepsilon .28$ & & Leas & $304559\left(+0.1^{\circ} 2 \mathrm{rrc}\right)$ & 3.5 & $\ldots$ & $\ldots$ & Sand-B & 9.65 & 538 & 9,800 & 1.200 & $3.0 \times 10^{4}$ & $2.9 \times 10^{5}$ \\
\hline
\end{tabular}

*Best Package designs from (Lester, 1979) and (Stula. 1980a) 
Table 5-2. Previous Best* Package Design Results with Current BARIER Model. (Continued)

\begin{tabular}{|c|c|c|c|c|c|c|c|c|c|c|c|c|c|}
\hline 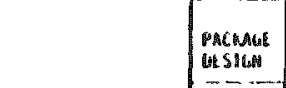 & $\begin{array}{lll}a b 5 & \text { mats }\end{array}$ & 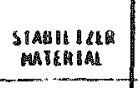 & $\begin{array}{l}\text { CAMBSISER } \\
\text { MARERAII } \\
\end{array}$ & 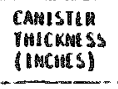 & 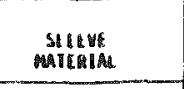 & 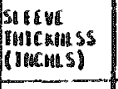 & 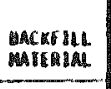 & 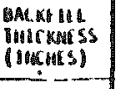 & $\operatorname{man}$ & Alacin bibil & 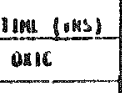 & 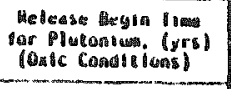 & 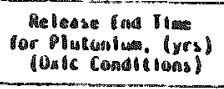 \\
\hline 0.1 & shale & Massst & yousss & 0.25 & $\cdots$ & $\cdots$ & $\operatorname{san} \theta-18$ & 13.0 & Sot & 1.100 & 350 & $29 \cdot 10^{8}$ & $2.8 \times 10^{5}$ \\
\hline a.s & & 304asst & 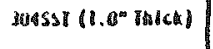 & 1.0 & $\cdots$ & $\cdots$ & Send-B & 2.25 & 943 & 8,800 & 2.9 and & 3.960 & $8.8 \times 10^{5}$ \\
\hline A. 10 & & Ztrcalay & $\begin{array}{l}\text { Zircafoy } \\
\text { Inisck) }\end{array}$ & 1.0 & $\cdots$ & $\cdots$ & Sonde-8 & 2.25 & 463 & 9.608 & 9.6000 & 8.006 & $9.1 \times 10^{5}$ \\
\hline 8.8 & & Steet & steel & 0.25 & $\begin{array}{l}\text { ficcatoy } \\
\text { fovervacks) }\end{array}$ & 1.0 & $\operatorname{sand}-8$ & 1.75 & 940 & 9,600 & 9.6000 & 8.600 & $9.2 \times 10^{5}$ \\
\hline 81. . & & Stee! & Steal & 0.25 & $\begin{array}{l}\text { Steel } \\
\text { Leady }\end{array}$ & 0.25 & c14no & 3.5 & soy & 4 & 3 & 2 & $3.7 \times 10^{5}$ \\
\hline 41..11 & & stee! & steal & a. 25 & 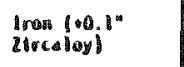 & 4.0 & Sosad-i -8 & 12.65 & 540 & 8.500 & 1,100 & $3.0=10^{8}$ & $2.2 \times 10^{5}$ \\
\hline c.7 & & Hes it wew & Eircedoy & 1.0 & $\begin{array}{l}\text { girccitoy } \\
\text { leverpac(s) }\end{array}$ & 1.0 & $S_{\text {Sand }}-8$ & 11.0 & 548 & 19,0000 & 19.00ande & $4.6 \times 10^{8}$ & $2.8 \times 10^{8}$ \\
\hline \$1.. & & Incume 1 & Inconel & 0.25 & $\begin{array}{l}\text { Iron (100.85" } \\
\text { Jusssy) over- } \\
\text { patcat) }\end{array}$ & 3.5 & Sond-8 & 11.25 & 538 & 8. & \#20 & $2.9 \times 10^{4}$ & $2.2 \times 10^{5}$ \\
\hline (1.). & & Hel tium & Incovere I & 0.25 & $\begin{array}{l}\text { Iron cou. 25" } \\
\text { Juasssi over" } \\
\text { wack }\end{array}$ & 3.5 & Sand-B & 11.25 & 540 & 1. & แ20 & $8.9 \times 10^{4}$ & $2.9 \times 10^{5}$ \\
\hline 0.6 & & enestay & estredtay & u. 25 & 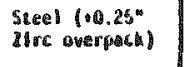 & 0.5 & Sand-B & 3.75 & 503 & 8.000 & 4.300 & 4.300 & $3.5 \times 10^{5}$ \\
\hline 0.1 & & Hell lium & Stuel & 0.25 & Bron & 3.5 & $\operatorname{Sand}-4$ & 13.25 & 549 & $\$ 20$ & 110 & $2.9 \times 10^{8}$ & $3.5 \times 10^{5}$ \\
\hline 0.1 & & stetel & steet & $0.2 \mathrm{~s}$ & Iron & 3.5 & Sond -a & 13.25 & 596 & 510 & 2000 & $2.8 \times 10^{8}$ & $3.5 \times 30^{5}$ \\
\hline 0.9 & & Steet & Stee! & 0.25 & Atrcalay & 1.0 & Sanat-a & 15.75 & 578 & 9.700 & 9.200 & $3.8 \times 10^{8}$ & $3.5=10^{5}$ \\
\hline DI.2 & & Masisy & suassi & 0.25 & Bow & 4.9 & Send - is & 12.75 & 598 & 2,0000 & $6 / 0$ & $2.8 \times 10^{8}$ & $3.5 \pm 110^{8}$ \\
\hline b1.5 & & seass & SUA3ST & 0.25 & 304ss I & 0.25 & Send-B & 2.5 & 890 & 2.6000 & 450 & 950 & $4.1 \times 10^{5}$ \\
\hline 02.8 & & Ins anel & Iancinet & - 25 & 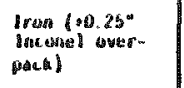 & 8.0 & Sotas - B & 82.28 & 580 & 690 & 170 & $2.8 \times 10^{4}$ & $6.4 \times 10^{5}$ \\
\hline vi, 8 & & Bircatioy & Lircatuy & 0.25 & 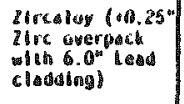 & 8.25 & citinas & 18.8 & 5 & 82.0020 & 18.0000 & $3.2 \times 180^{4}$ & 2.8 $a 6^{8}$ \\
\hline 6.3 & & Lead & 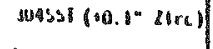 & 0.25 & $\cdots$ & $\cdots$ & SABA-B & 8.9 & 492 & ه. 0003 & 2.0000 & 8.4010 & 3.9 a 105 \\
\hline 8.4 & & Lead & dircolug & 0.25 & $\cdots$ & $\cdots$ & Sansu- & 3.0 & 494 & 3.000 & 3. 490 & 3.3004 & $3.2 \times 14^{5}$ \\
\hline 1.24 & .. & teasu & 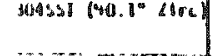 & 9.5 & $\cdots$ & $\cdots$ & Sand- B & 9.85 & 321 & 37,000010 & 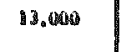 & $8.1 \times 10^{8}$ & $2.8 \times 10^{5}$ \\
\hline
\end{tabular}

*Best Package desianns from (Lester, 1979) and (Stula, 1980a) 
Table 5-2. Previous Best* Package Design Results with Current BARIER Model. (Continued)

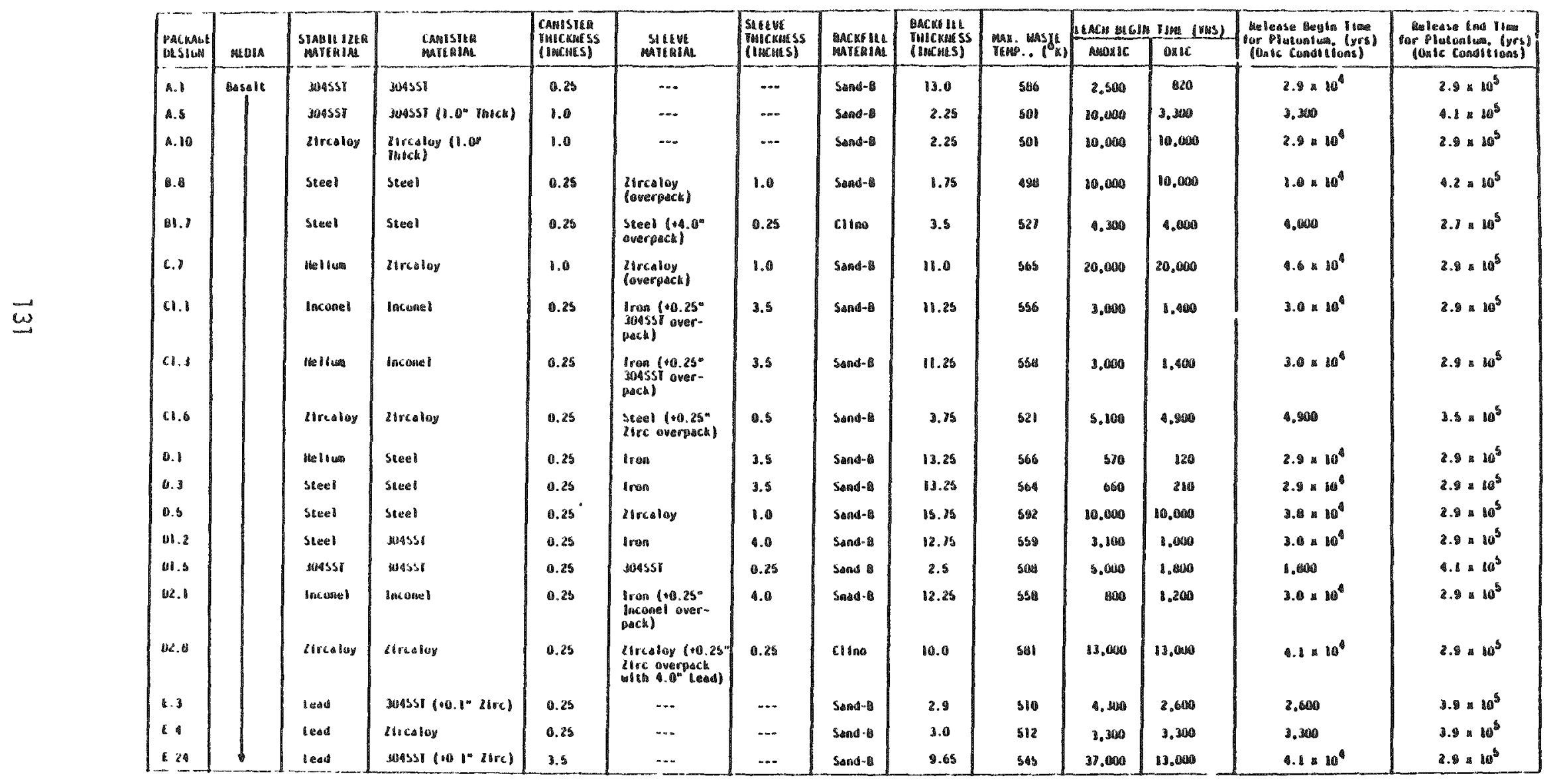


Table 5-3. Best Package Designs in Current Study (0xic Conditions).

\begin{tabular}{|l|l|c|c|c|}
\hline Geology & $\begin{array}{l}\text { Package } \\
\text { Design }\end{array}$ & $\begin{array}{c}\text { Leach Begin } \\
\text { Time, (yrs) }\end{array}$ & $\begin{array}{c}\text { Release Begin Time } \\
\text { for Plutoniuin, (yrs) } \\
\text { (0xic Conditions) }\end{array}$ & $\begin{array}{c}\text { Release End Time } \\
\text { for Plutonium, (yrs) } \\
\text { (0xic Conditions) }\end{array}$ \\
\hline Salt & $\begin{array}{l}\text { BE.12N, } \\
\text { BE.25N }\end{array}$ & 5,000 & $3.3 \times 10^{4}$ & $2.9 \times 10^{5}$ \\
B1.18N & 29,000 & $5.4 \times 10^{4}$ & $2.8 \times 10^{5}$ \\
& E.9N & 30,000 & $8.1 \times 10^{4}$ & $2.9 \times 10^{5}$ \\
& Bl.21N & 2,600 & $3.0 \times 10^{4}$ & $2.8 \times 10^{5}$ \\
& BE.25N & 5,900 & $3.4 \times 10^{4}$ & $2.9 \times 10^{5}$ \\
Basalt & E.13N & 3,800 & $3.2 \times 10^{4}$ & $2.9 \times 10^{5}$ \\
& Bl.9N & 12,000 & $6.7 \times 10^{4}$ & $2.9 \times 10^{5}$ \\
BE.12N & 5,900 & $3.4 \times 10^{4}$ & $2.9 \times 10^{5}$ \\
\hline
\end{tabular}


Table 5-4. Concept E Results in Current Study.

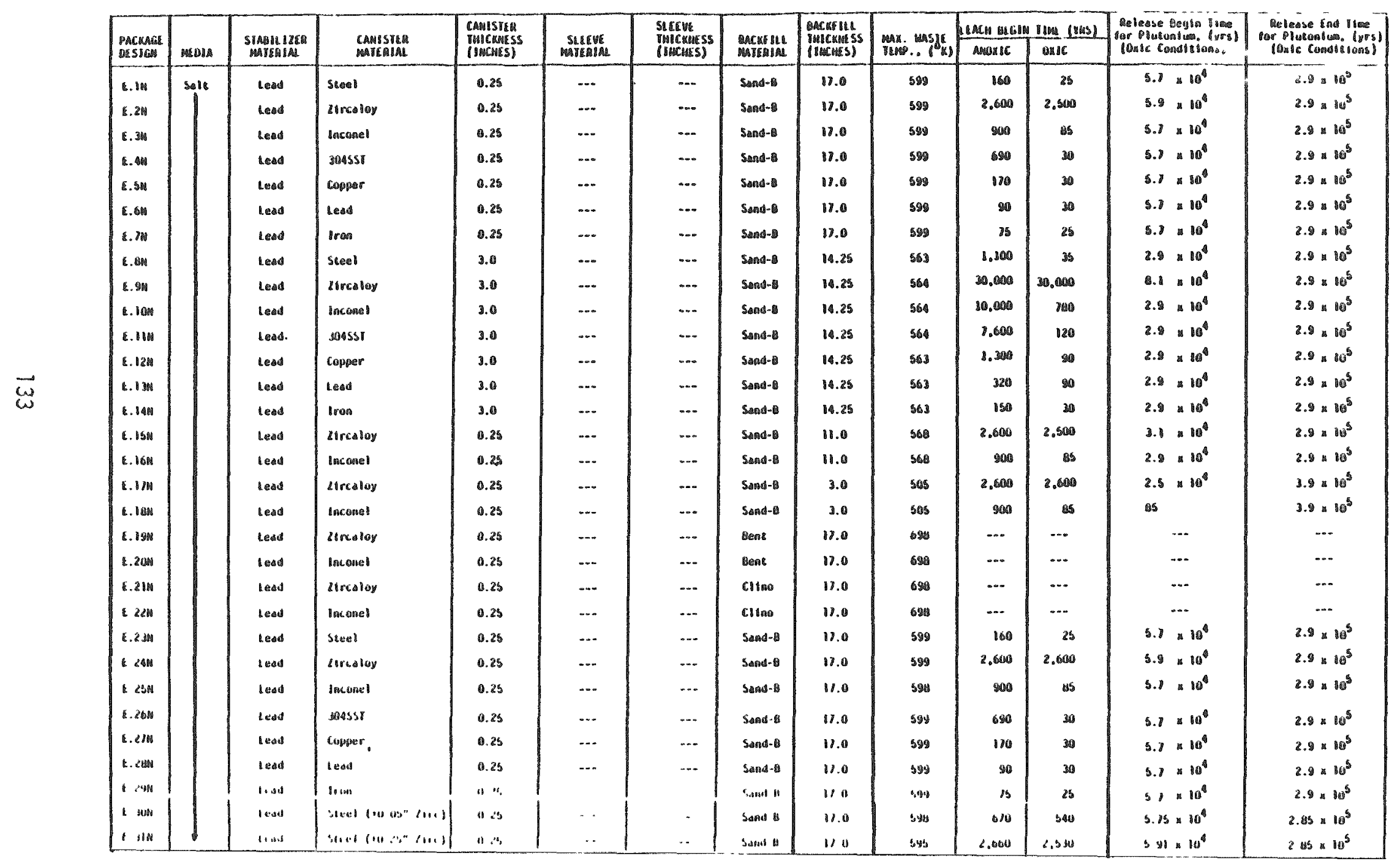


Table 5-4. Concept E Results in Current Study. (Continued)

\begin{tabular}{|c|c|c|c|c|c|c|c|c|c|c|c|c|c|}
\hline 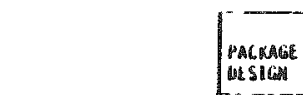 & itit mesas & 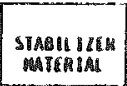 & 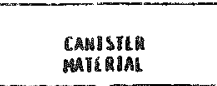 & 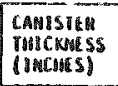 & 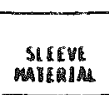 & 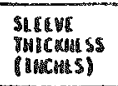 & 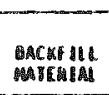 & 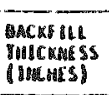 & 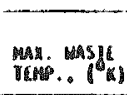 & 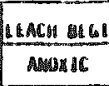 & 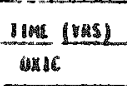 & 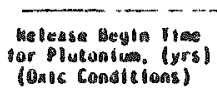 & 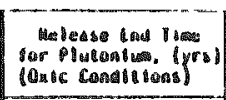 \\
\hline $6.12 \mathrm{~m}$ & \begin{tabular}{l|l}
$*$ & Shalls \\
\end{tabular} & ieas & copper & 3.0 & $\cdots$ & ... & Sonst-ie & 18.25 & s52 & 2,300 & 2.300 & $3.1 \times 10^{4}$ & $2.9 \times 10^{5}$ \\
\hline 6.03m & \begin{tabular}{l|l}
31 & Smale
\end{tabular} & Lead & Lead & 3.0 & $\cdots$ & $\ldots$ & Sensw-18 & 14.25 & $\$ 52$ & 3,800 & 3,4000 & $1.2 \times 10^{4}$ & $2.9 \times 10^{5}$ \\
\hline $8.09 \mathrm{ur}$ & \begin{tabular}{l|l} 
In & Shasile
\end{tabular} & Lead & trean & 3.0 & $\cdots$ & $\ldots$ & Sarad a & 18.25 & -552 & 1.000 & ass & $2.9 \times 10^{4}$ & $2.8 \times 10^{5}$ \\
\hline \& & \begin{tabular}{l|l} 
in & shalle \\
\end{tabular} & Ieas & Zircalay & 0.25 & ... & $\cdots$ & Sand-A & 11.0 & 558 & 3,300 & 3.000 & $3.2 \times 10^{4}$ & $2.8 \times 10^{5}$ \\
\hline 8.3 & Salts & tead & $30455 \mathrm{~T}\left(\cdot 0.1^{\mathrm{m}} \mathrm{Z \textrm {irc }}\right)$ & 0.26 & $\cdots$ & -.. & Sana-B & 2.9 & 503 & 3.000 & 8.000 & 1.0000 & $3.9 \times 10^{5}$ \\
\hline 6.3 & Sthale & Iead & Sousss (00.1" z1rc) & 0.25 & $\cdots$ & --. & Sand-1-8 & 2.9 & ه92 & 4.000 & 3.600 & 2.600 & $3.9 \times 10^{5}$ \\
\hline 6.3 & cossols & teos & 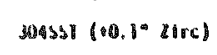 & 0.25 & $\ldots$ & .-. & Sond-a & 2.9 & Sto & 4.3000 & 2.6000 & 2.6000 & $5.9 \times 10^{3}$ \\
\hline E.4 & solt & Lead & 2trcallay & 0.25 & $\ldots$ & $\cdots$ & Sand-a & 3.0 & sos & 2.000 & 3.5000 & 2.5000 & $3.9 \mathrm{a}=0^{5}$ \\
\hline t. 4 & Shale & teed & Zirualay & 0.25 & $\cdots$ & $\ldots$ & Sand-B & 3.0 & 494 & $B_{0}$ & 3.,000 & 3,000 & $3.9 \times 10^{5}$ \\
\hline E. 4 & Basalt & Lead & Lirresloy & 0.25 & $\ldots$ & $\ldots$ & SaAd-B & 3.0 & $\$ 12$ & 3.000 & 3.000 & 3.300 & $3.9 \times 10^{8}$ \\
\hline 8.24 & salt & Lesas & Massst (100.10" $2 \mathrm{rrc})$ & 0.25 & -.. & $\cdots$ & Sans-B & 9.05 & s3e & 9.,wa & 1.2000 & $3.8 \times 10^{4}$ & $2.8 \times 100^{5}$ \\
\hline t. 24 & Shale & Lesu & s0455 ( $\left.+0.10^{\circ} \mathrm{Zirc}\right)$ & 0.25 & $\ldots$ & ... & Sand-18 & 9.65 & 328 & 37,0000 & 13,0000 & $8.1 \times n 0^{4}$ & $2.9 \times 10^{5}$ \\
\hline 1.4 & Sasalt & Lead & $30035 s\left[0.10^{n} \mathrm{arrc}\right)$ & 0.23 & $\cdots$ & --- & Sand-8 & 9.65 & s45 & 33,0000 & 13.0000 & $9.2 \times 10^{4}$ & $2.9 \times 10^{5}$ \\
\hline
\end{tabular}


thickness was found to have a negligible effect on leach begin time. The use of the cast solid stabilizer provides sufficient package strength to make the effects of media crushing forces relatively smal1. Of the canister materials tested, Zircaloy canisters or canisters of other metals clad with Zircaloy were found to give the longest leach begin times. This is due primarily to the relatively small corrosion rates for Zircaloy.

As can be seen in Table 5-4, the radionuclide release breakthrough is delayed an enormous amount of time when appropriate backfill is used.

\subsection{HEAVY SLEEVE CONCEPT (Concept B1)}

Concept BI originally consisted of a mild steel canister surrounded by a heavy sleeve designed to withstand high creep rate media crushing forces. In addition to the heavy sleeve, a backfill cushion was included for additional support. A protective sleeve cladding is al so included in certain cases to increase the corrosion resistance of the sleeve. Previous work (Lester, 1979) had shown that backfill thickness had little or no effect on package performance but that sleeve design was significant in creeping media.

In addition, it was shown that of the materials considered for a sleeve cladding material, Zircaloy provided the best resistance. In the current study, sleeve material and thickness as well as sleeve cladding thickness are varied.

Results of the calculations for the Concept B1 package design variations are tabulated in Table 5-5. Conclusions that can be drawn from the calculations are consistent with those reported in (Stula, 1380a). That is, sleeve cladding thickness is significant only in those cases where sleeve thickness exceeds a minimum thickness. Corrosion resistance afforded by the cladding is inconsequential unless the sleeve is able to withstand media crushing forces. Of the materials tested, the best combinations consist of a zircaloy cladding with a 304 SST sleeve. Calculated leach begin times in salt are generally less than corresponding times in shale which are, in turn, less than those in basalt. This is due to the high creep rate in salt and the negligible creep rate assumed in basalt.

As with the Econcept, when a backfill is used, radionuclide release occurs at very long times after package failure, is attenuated by a large factor and is spread out over very long times. 
Table 5-5. Concept B1 Results in Current Study.

\begin{tabular}{|c|c|c|c|c|c|c|c|c|c|c|c|c|c|}
\hline $\begin{array}{l}\text { PackaGs } \\
\text { OESSIGA }\end{array}$ & B.,OO1A & 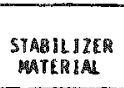 & 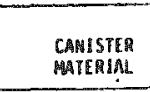 & 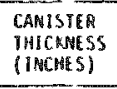 & $\begin{array}{c}\text { SLEEVE } \\
\text { Mareriat }\end{array}$ & 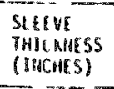 & $\begin{array}{l}\text { Backh IL } \\
\text { materiat }\end{array}$ & 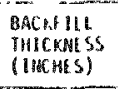 & 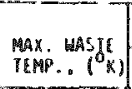 & $\frac{\text { LEALA BEG }}{\text { ANDXX }}$ & $\begin{array}{l}\text { TIAAE (YNS) } \\
\text { URIC }\end{array}$ & 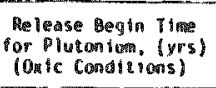 & 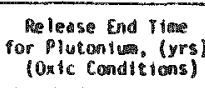 \\
\hline 81. 11 & Sols & Staeal & Stmet & 0.25 & Iron & 0.25 & Sand-B & 16.5 & 595 & 2 & 2 & $2.8 \times 10^{6}$ & $2.8 \times 10^{5}$ \\
\hline O1. $2 \mathrm{~W}$ & & steel & seeel & 0.25 & Iron & 3.5 & Sand-B & 13.25 & 557 & 55 & 9 & $2.8 \times 10^{4}$ & $2.8 \times 10^{5}$ \\
\hline B1. 3 .36 & & Stwel & stoel & 0.25 & iron & 6.0 & Sand-B & 10.75 & 539 & 120 & 18 & $2.8 \times 10^{4}$ & $2.8 \times 10^{5}$ \\
\hline 81. 49 & & Stoel & steel & 0.25 & $\left(\begin{array}{l}\text { ron } \\
(+0.05 " \mathrm{zirc})\end{array}\right.$ & 3.5 & Sand-B & 13.2 & 556 & 560 & 520 & $2.8 \times 10^{4}$ & $2.8 \times 100^{5}$ \\
\hline $81.5 \mathrm{~m}$ & & Steot & steel & 0.25 & 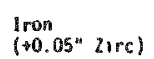 & 3.5 & Sand-B & 13.1 & 555 & 1,600 & 1,500 & $2.9 \times 10^{4}$ & $2.8 \times 10^{5}$ \\
\hline 81. $6 \mathrm{M}$ & & steel & Steel & 0.25 & 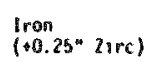 & 3.5 & Sand-B & 13.0 & $\$ 54$ & 2,600 & 2.500 & $3.0 \times 10^{8}$ & $2.8 \times 10^{5}$ \\
\hline B1. $2 \mathrm{~N}$ & & Steet & Steel & 0.25 & $\begin{array}{l}\text { Iren } \\
(+0.15 " \text { zirc })\end{array}$ & 0.25 & Sand-B & 16.35 & 593 & 10 & 10 & $2.8 \times 10^{4}$ & $2.8 \times 10^{5}$ \\
\hline 81.8ल & & Steel & Steel & 0.25 & $\left.\operatorname{lran}_{\left(+0.15^{n}\right.} 2 \mathrm{irc}\right)$ & 6.0 & Sanda-B & 10.6 & 5.33 & 1.600 & 1.500 & $2.9 \times 10^{8}$ & $2.8 \times 10^{5}$ \\
\hline B1. 19 & & Steel & Steel & 0.25 & $\begin{array}{l}3045 \mathrm{SI} \\
\left(+0.05^{\circ} \mathrm{LirC}\right)\end{array}$ & 3.5 & Sand-B & 13.2 & 557 & 6.400 & 590 & $2.8 \times 10^{4}$ & $2.8 \times 10^{5}$ \\
\hline 18. $10 \mathrm{w}$ & & Stees & stees & 0.25 & $\begin{array}{l}3095 S \mathrm{SI} \\
(+0.05 " \text { larc })\end{array}$ & 3.5 & jand-B & 13.0 & 555 & 8.500 & 2.600 & $3.0 \times 10^{4}$ & $2.8 \times 10^{5}$ \\
\hline 81. $1 \mathrm{M}$ & & Steel & Steel & 0.25 & Copper & 0.25 & Send-B & 16.5 & 595 & 2 & 2 & $2.8 \times 10^{8}$ & $2.8 \times 10^{5}$ \\
\hline 81. $122 \%$ & & Steel & Steel & 0.25 & copper & 3.5 & Sand-B & 13.25 & 556 & 2 & 2 & $2.8 \times 10^{4}$ & $2.8 \times 10^{5}$ \\
\hline $81.13 \mathrm{~m}$ & & Steer & Steel & 0.25 & Incomel & 0.25 & Sand-B & 16.5 & 595 & 2 & 2 & $2.8 \times 10^{4}$ & $2.8 \times 10^{5}$ \\
\hline B1. 194 & & Stee I & Steel & 0.25 & Inconel & 3.5 & Sand-B & 13.25 & 557 & 8.100 & 600 & $2.8 \times 10^{8}$ & $2.8 \times 10^{5}$ \\
\hline $81.15 \%$ & & Stee I & Streel & 0.25 & 30ASST & 0.25 & Sand-B & 16.5 & 595 & 2 & 2 & $2.8 \times 10^{4}$ & $2.8 \times 10^{5}$ \\
\hline $81.16 \mathrm{~m}$ & & Steel & Stee) & 0.25 & 304SST & 3.5 & Sand-B & 13.25 & 557 & 5,900 & 83 & $2.8 \times 10^{4}$ & $2.8 \times 10^{5}$ \\
\hline $81.17 \mathrm{~W}$ & & Steel & steel & 0.25 & Zircaloy & 0.25 & Sand-8 & 16.5 & 595 & 10 & 10 & $2.8 \times 10^{4}$ & $2.8 \times 10^{5}$ \\
\hline $01.18 \mathrm{~m}$ & & steet & Stee? & 0.25 & Lircaloy & 3.5 & Sond-8 & 13.25 & 557 & 29,000 & 29,000 & $5.4 \times 10^{4}$ & $2.8 \times 10^{5}$ \\
\hline 81. $19 \mathrm{gm}$ & & stee I & Stea 1 & 0.25 & 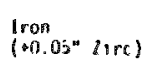 & 3.5 & Sand-B & 13.2 & 556 & 360 & 520 & $2.8 \times 10^{4}$ & $2.8 \times 10^{5}$ \\
\hline $81.20 \mathrm{~m}$ & & Steel & Steel & 0.25 & $\begin{array}{l}\operatorname{lran} \\
(+0.15 " 21 \times())\end{array}$ & 3.5 & Sand-8 & 13.1 & 355 & 1.600 & 1,500 & $2.9 \times 10^{4}$ & $2.8 \times 10^{5}$ \\
\hline (11.216 & 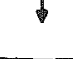 & steel & Steel & 0.25 & 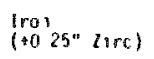 & 3.5 & Sand-B & 13.0 & 554 & 2.000 & 2,500 & $3.0 \times 10^{4}$ & $2.8 \times 10^{5}$ \\
\hline
\end{tabular}


Tab7e 5-5. Concept BI Results in Current Study. (Continued)

\begin{tabular}{|c|c|c|c|c|c|c|c|c|c|c|c|c|c|}
\hline PACKaCde & & STABHLIZER & CANISRER & $\begin{array}{l}\text { CANISIER } \\
\text { TMICNAESSS }\end{array}$ & Stettr & $\begin{array}{l}\text { SAlIVE } \\
\text { InIt kAlESS }\end{array}$ & Bachoflit & $\begin{array}{l}\text { SACKIILL } \\
\text { IMLKNESS }\end{array}$ & & LEACH BEGIR & InL (ons) & $\begin{array}{l}\text { Helease begin T, } \\
\text { for Plutionsum, (yrs) }\end{array}$ & $\begin{array}{l}\text { Re lease Ind Ther } \\
\text { for pluten lum, (yrs) }\end{array}$ \\
\hline DE SIGM & MEIA & MATERIAI & MAILAINL & & MAYK RIAL & & MaItR!AL & & TEME, . . $\left.{ }^{\circ} \mathrm{K}\right)$ & ANOKIC & ONAC & & \\
\hline 81.2218 & Solt & Steel & Steel & 0.25 & $\begin{array}{l}\text { Iron } \\
\left(00.15^{\prime \prime}\right. \\
\mathrm{arc})\end{array}$ & 0.25 & Sande-B & 16.35 & 593 & 2 & 2 & $2.8 \times 10^{4}$ & $2.8 \times 10^{5}$ \\
\hline $81.23 \mathrm{M}$ & & steel & steel & 0.25 & $\begin{array}{l}\text { fron } \\
\left(00.15^{\circ} 21 \mathrm{rc}\right)\end{array}$ & 6.0 & Sand-8 & 10.25 & 533 & 1.600 & 1.500 & $2.9 \times 10^{4}$ & $2.8 \times 10^{5}$ \\
\hline 81.284 & & Hellu & Steel & 0.25 & Iron & 3.5 & Sond-8 & 13.25 & 559 & 55 & 9 & $2.8 \times 10^{4}$ & $2.8 \times 10^{5}$ \\
\hline $81.25 \mathrm{NA}$ & & Ajp & steel & 0.25 & Iron & 3.5 & Sand -8 & 13.25 & 560 & $5 s$ & 9 & $2.8 \times 10^{8}$ & $2.8 \times 10^{5}$ \\
\hline $01.26 \mathrm{~m}$ & & Het f iven & $\begin{array}{l}\text { Steel (00.25" } \\
\text { Air gap) }\end{array}$ & 0.25 & Iron & 3.5 & $\operatorname{sand}-8$ & 13.25 & 559 & 55 & 9 & $2.8 \times 10^{4}$ & $2.8 \times 10^{5}$ \\
\hline $81.27 \%$ & & Melium & Steel ( $+0.5^{\prime \prime}$ Air $\left.\left.9 \partial_{t}\right)^{\prime}\right)$ & 0.25 & Iron & 3.5 & Sand-B & 13.25 & 558 & 54 & 9 & $2.8 \times 10^{8}$ & $2.8 \times 10^{5}$ \\
\hline $81.20 \%$ & & steel & Steel ( $+1.0^{\prime \prime}$ arr gap $)$ & 0.25 & Iron & 3.5 & Sand-B & 33.25 & 552 & 52 & 8 & $2.8 \times 10^{8}$ & $2.8 \times 10^{5}$ \\
\hline 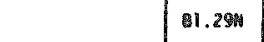 & 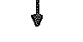 & Steel & Stee $\left(+2.0^{\circ}\right.$ Air gap $)$ & 0.25 & Iron & 3.5 & Sand-8 & 13.25 & 548 & 48 & 8 & $2.8 \times 10^{4}$ & $2.8 \times 10^{5}$ \\
\hline $81.20 \mathrm{~N}$ & Shale & Steel & Steel & 0.25 & 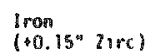 & 3.5 & Sand-8 & 13.1 & 544 & 1,800 & 1,600 & $2.9 \times 10^{8}$ & $2.8 \times 10^{5}$ \\
\hline $81.21 \%$ & & Steel & Steel & 0.25 & $\begin{array}{l}\text { Iron } \\
(00.25 " 24 r c)\end{array}$ & 3.5 & Sand-6 & 13.0 & 543 & 2.800 & 2.690 & $3.0 \times 10^{8}$ & $2.8 \times 10^{5}$ \\
\hline $01.22 \mathrm{~N}$ & & steel & Steel & 0.25 & $l_{\left(+0.15^{\prime \prime}\right.}(1 \mathrm{rc})$ & 0.25 & Sand-B & 15.85 & 382 & 2 & 2 & $2.8 \times 10^{8}$ & $2.0 \times 10^{5}$ \\
\hline 31.239 & $t$ & Steel & Steel & 0.25 & $\begin{array}{l}\text { Iron } \\
\left(+0.15^{\circ} 2 \mathrm{rre}\right)\end{array}$ & 6.0 & Sand-B & 10.6 & 522 & 2.100 & 1,700 & $2.9 \times 10^{4}$ & $2.8 \times 10^{5}$ \\
\hline 81.11 & Rasalt & Steel & Stee 1 & 0.25 & Iron & 0.25 & Sand-B & 16.5 & 602 & 160 & 20 & $2.9 \times 10^{8}$ & $2.9 * 10^{5}$ \\
\hline 81.76 & & Steel & Steel & 0.25 & $\begin{array}{l}\text { lron } \\
(10.15 " \mathrm{lrc})\end{array}$ & 0.25 & Sand-8 & 16.35 & 600 & 1.700 & 1,500 & $3.0 \times 10^{4}$ & $2.9 \times 10^{5}$ \\
\hline 81.99 & & Steel & Stee 1 & 0.25 & $\begin{array}{l}\text { 3nass sy } \\
(00.05 \text {, lirc) }\end{array}$ & 3.5 & SanduB & 13.2 & 564 & 36,000 & 12,000 & $6.7 \times 10^{4}$ & $2.9 \times 10^{5}$ \\
\hline $81.11 \%$ & & Stee 1 & steer & 0.25 & Copper & 0.25 & Sond-8 & 16.5 & 602 & 240 & 130 & $2.9 * 10^{4}$ & $2.9 \times 10^{5}$ \\
\hline $81.12 \mathrm{M}$ & & steel & steel & 0.25 & Copper & 3.3 & Sand-18 & 13.25 & 563 & 1.900 & 1,800 & $3.0 \times 10^{4}$ & $2.9 \times 10^{5}$ \\
\hline 81.739 & & Steel & steel & 0.25 & Inconel & 0.25 & Sand-8 & 16.5 & 602 & 220 & 510 & $2.9 \times 10^{8}$ & $2.9 \times 10^{5}$ \\
\hline 81. $14 \mathrm{~N}$ & & Steel & Steel & 0.25 & Inconel & 3.5 & Sand-8 & 13.25 & 564 & 1.500 & 7.000 & $6.3 \times 10^{4}$ & $2.9 \times 10^{5}$ \\
\hline $81.15 \mathrm{~A}$ & & Steel & Steel & 0.25 & $30453 \pi$ & 0.25 & Sand-18 & 10.5 & 602 & 2.600 & 840 & $2.9 \times 10^{4}$ & $2.9 \times 10^{5}$ \\
\hline $01.1 / \mathrm{M}$ & & Stee I & Steel & 0.25 & zirealoy & 0.25 & Sand-B & 10.5 & 602 & 2,600 & 2.500 & $5.9 \times 10^{4}$ & $2.9 \times 10^{5}$ \\
\hline $81.22 \%$ & 1 & Steel & Steel & 0.25 & $\begin{array}{l}\text { lron } \\
(+0) 15^{\prime \prime}(1, m)\end{array}$ & 0.25 & Sand-6 & 16.35 & 6000 & 1.700 & 1.500 & $3.0 \times 10^{8}$ & $2.9 \times 10^{5}$ \\
\hline
\end{tabular}


5.4 HEAVY SLEEVE/CAST STABILIZER CONCEPT (Concept BE)

A package design utilizing both a solid cast stabilizer and a heavy sleeve had deen thought to be an attractive concept on the basis of results of previous work (Stula, 1980a). Therefore, variations of this design (Concept BE) are evaluated in the current study. Calculations are perfomed primarily in creeping geologic media with most of the cases evaluated in sait. Results of calculations for the Concept $B E$ design variations are presented in Table 5-6.

Comparison of package designs varying only in canister material show a large variance in leach begin time which indicates a significant dependence on corrosion rate. As in the concept B1 package design, sleeve thickness is sufficient to prevent immediate crushing of the package. With sufficient sieeve strength, corrosion resistance of the sleeve cladding is important in determining package lifetime. Backfill thickness was found to have no effect on leach begin time. Calculated leach begin times in salt are jenerally much less than corresponding times in shale and basalt.

As in all other designs the backfill greatly delays and attenuates radionuclide release.

\subsection{PEAK WASTE TEMPERATURE}

Use of design packages with many layers and/or low conductivity materials could result in very high waste temperatures. A maximum temperature criterion of $653^{\circ} \mathrm{K}\left(380^{\circ} \mathrm{C}\right)$ is used to reject package designs. Calculated peak waste temperatures for all package designs evaluated are included in Tables 5-2, $5-4,5-5$, and $5-8$. of the design cases evaluated, only four (E.19il - E.22N) exceed the maximum temperature criterion.

The package design characteristics having the most pronounced effect on peak waste temperature are type of backfill material and backfill thickness. The effect of type of backfill material is shown in Table 5-7 where use of backfill naterials with relatively low thermal conductivities sucn as bentonite and clinoptilolite result in higher peak waste temperatures than in the case when sand-bentonite (10 percent) is used. The effect of backfill thickness is al so shown in Table 5-7. Peak waste temperature increases significantly with increasing backfill thickness. Types of barrier wall materials (metals) and barrier wall thicknesses have little, if any, effect on peak waste temperature. 
Table 5-6. Concept BE Results in Current Study.

\begin{tabular}{|c|c|c|c|c|c|c|c|c|c|c|c|c|c|}
\hline $\begin{array}{l}\text { Packact } \\
\text { persicos }\end{array}$ & MEOIA & 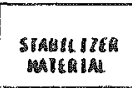 & 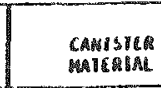 & 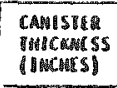 & $\begin{array}{c}\text { SLLEUE } \\
\text { matenatal }\end{array}$ & 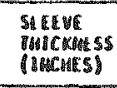 & 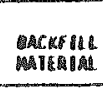 & 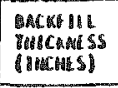 & 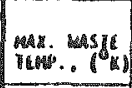 & HaAcu utc & Joms (bus) & 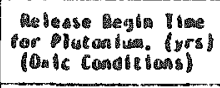 & 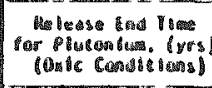 \\
\hline Br. . 18 & Solt: & Lead & 300ssi & 0.25 & Pron & 0.25 & SonA-B & 16.5 & S9g & 2000 & 3 & $3.9=10^{8}$ & $2.8 \times 10^{6}$ \\
\hline $8 \mathrm{t} .2 \mathrm{M}$ & & Lead & 3045s & 0.25 & Iron & 3.5 & Sand -18 & 13.25 & 958 & 290 & 50 & $2.9: 80^{6}$ & $2.9 \mathrm{n} B 0^{5}$ \\
\hline Bt. 3m & & Lead & 304ss5 & 0.25 & Iron & 6.0 & Sand-B & 10.75 & 536 & 860 & so & $2.9 \times 10^{4}$ & $2.9 \times 10^{5}$ \\
\hline Be. .96 & & Lead & 304sst & 0.25 & $\left(+0.05^{\circ} \mathrm{Ztre}\right)$ & 3.5 & Sand-B & 13.2 & 556 & 1,300 & 558 & $2.9 \times 10^{8}$ & $2.9 .810^{5}$ \\
\hline eE. $5 \mathrm{~m}$ & & leses & 304558 & 0.25 & Iron & 3.5 & Sond-i & 13.0 & 558 & 3.900 & 3.600 & $3.8 \times 10^{6}$ & $2.8 \times 10^{5}$ \\
\hline BE. ...M & & Lead & 30osst & 0.25 & fros & 8.25 & Sende-8 & 16.38 & 593 & 2.200 & 1.500 & $3.0 \times 10^{4}$ & $2.9 \times 10^{8}$ \\
\hline 86. 20 & & Lead & 3045s & 0.25 & 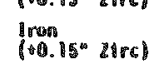 & 6.0 & $\sin \theta-1$ & 10.6 & 533 & 2.480 & 1.600 & $3.0 \div 30^{6}$ & $2.9 \times 10^{5}$ \\
\hline Wat.am & & ledd & Ilircsioy & 0.25 & Irows & 0.25 & Sand-8 & 16.5 & 595 & 2.600 & 2.500 & $5.9 \times 10^{4}$ & $2.9 \times 180^{5}$ \\
\hline OBi . $9 \mathrm{~m}$ & & lead & Iincaloy & 0.25 & Iron & 3.5 & Sando- $-\mathbb{B}$ & 13.25 & 558 & 2.300 & 2.5000 & $3.3 \times 10^{\circ}$ & $2.8 \times 10^{5}$ \\
\hline BE. & & lead & Rorcaloy & 0.25 & Iron & 0.0 & Send-B & 10.75 & $\$ 38$ & 2.700 & 3.500 & $3.8 \leq 10^{8}$ & $2.9 \times 10^{8}$ \\
\hline Ot. $11 \mathrm{~N}$ & & lead & bircaloy & 0.28 & $\begin{array}{l}\text { Iron } \\
\left(+0.05^{*} 21(r c)\right.\end{array}$ & 3.5 & Sand-B & 13.8 & 536 & 3.200 & 3,0000 & $3.1 \times 10^{\circ}$ & $2.9 \times 10^{5}$ \\
\hline BE. $.12 \mathrm{M}$ & & iead & Burcaloy & 0.25 & $\left(\begin{array}{l}\text { ran } \\
\left(+0.25^{\prime \prime} \mathrm{Zluc}\right)\end{array}\right.$ & 3.5 & Sond-18 & 03.0 & 538 & 5,200 & 5,000 & $3.3 \times 10^{6}$ & $2.9 \times 1 e^{5}$ \\
\hline $68.13 \mathrm{M}$ & & leoul & elrculay & 0.25 & Irow & e.25 & Sand : & 16.35 & 593 & 8.100 & 9.000 & $3.2 \times 10^{\circ}$ & $2.8418 e^{5}$ \\
\hline $86 . .94 \mathrm{~m}$ & & Lead & aircestoy & 0.25 & 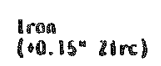 & 6.0 & Sanda- $-\mathbb{B}$ & 10.6 & 533 & 8.2400 & D.ounce & $3.8 \times 10^{8}$ & $2.9 \times 10^{5}$ \\
\hline Het $15 \mathrm{~A}$ & & trads & \begin{tabular}{|l|} 
Iaccunea \\
\end{tabular} & 0.25 & Iran & 0.25 & Sonst-B & 16.5 & s95 & 910 & $\$$ & $2.9 \times 10^{\circ}$ & $2.9 \times 10^{5}$ \\
\hline $8 \mathrm{Br} .186 \mathrm{NB}$ & & Lead & \begin{tabular}{|l} 
Incunel \\
\end{tabular} & 0.25 & Bren & 3.5 & Sand-B & 13.25 & 558 & 8.000 & wo & $2.9 \times 10^{4}$ & $3.9 \pm 10^{5}$ \\
\hline Jut $18 \mathrm{M}$ & & Lead & Intunel & 0.25 & Iran & 6.0 & Sonde-8 & 10.25 & 536 & 8.1000 & Inte & $2.8 \mathrm{a} 10^{4}$ & $29 \times 10^{5}$ \\
\hline | & & lead & \begin{tabular}{|l|l} 
Inc annel \\
\end{tabular} & 0.25 & $\begin{array}{l}\text { Iran } \\
(00.05 " \text { and }\end{array}$ & 8.5 & Sand-B & 13.2 & 336 & 1.5000 & 618 & $2.9 \times 10^{6}$ & $2.8 \times 10^{5}$ \\
\hline aE. $39 \mathrm{~N}$ & & leas & incuned & 0.25 & $\begin{array}{l}\text { Iren } \\
(+0.25 " 2 \mathrm{rrs})\end{array}$ & 3.5 & Sanu $\mathrm{A}$ & 13.0 & sso & 3.500 & 2.600 & $3.1 \times 100^{4}$ & $2.9810^{5}$ \\
\hline Wt. $20 \mathrm{~N}$ & & Lead & \begin{tabular}{|l} 
Inc aneel \\
\end{tabular} & 0.25 & 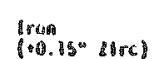 & 0.25 & Sander-8 & 16.35 & 593 & 2.8000 & 1,6000 & $3.0 \times 10^{8}$ & $2.8 \times 10^{5}$ \\
\hline WE $21 \mathrm{~W}$ & & I sast & Inaconel & 0.25 & $\begin{array}{l}\text { Iron } \\
(\because 0.15 " \mathrm{zirc})\end{array}$ & 6.0 & Sand - - & 10.6 & 533 & 2.6010 & 1.600 & $3.0 \times 10^{8}$ & $2.9=10^{5}$ \\
\hline
\end{tabular}


Table 5-6. Concept BE Results in Current Study. (Continued)

\begin{tabular}{|c|c|c|c|c|c|c|c|c|c|c|c|c|c|}
\hline 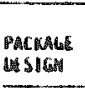 & Metals & 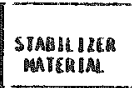 & $\begin{array}{l}\text { Camsister } \\
\text { Margasal. }\end{array}$ & 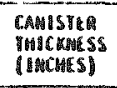 & $\begin{array}{c}\text { SHEvens } \\
\text { maverama }\end{array}$ & 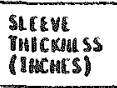 & 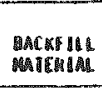 & 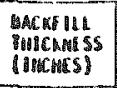 & 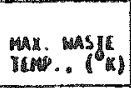 & 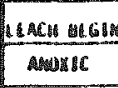 & oric (rus) & 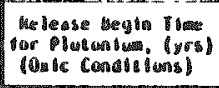 & 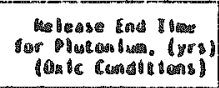 \\
\hline at. $22 \mathrm{z}$ & salt & Lead & zircaley & B. 28 & Iran & 3.5 & Sand-B & 13.25 & 558 & 2,100 & 3.5000 & $3.1 \times 10^{4}$ & $8.9 \mathrm{a}^{15} \mathrm{x}^{5}$ \\
\hline 8t.23m & & tead & zercalay & 0.25 & Iron & 6.0 & Send-B & 10.75 & 534 & 8.000 & 2.5000 & $3.1 \times 10^{8}$ & $8.9 \times 10^{5}$ \\
\hline BE. 24m & & teav & Burcaloy & 0.25 & $\begin{array}{l}\text { Irome } \\
\left(60.05^{\circ}\right. \\
\text { IIIrE) }\end{array}$ & 3.5 & Sona-8 & 13.2 & 556 & 3.200 & 3.040 & $3.1 \times 10^{8}$ & $8.9 \times 10^{5}$ \\
\hline ه6. $25 \mathrm{M}$ & & Lead & alcusloy & 0.25 & $\begin{array}{l}\text { Iron } \\
(+0.25 " \mathrm{strC})\end{array}$ & 3.5 & Sand-B & 13.0 & SA4 & 5.2000 & 5.0ure & $3.3 \times 10^{8}$ & $2.8 \times 180^{5}$ \\
\hline 86. $260 \mathrm{M}$ & & Lead & MASST & 0.25 & sron & 3.5 & Bent & 13.25 & 624 & 790 & 50 & $\cdots$ & $\cdots$ \\
\hline 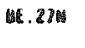 & & Lead & 3045st & 0.25 & 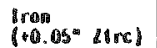 & 3.5 & Bents & 13.2 & 623 & 0.300 & 250 & $\cdots$ & $\cdots$ \\
\hline 86. $28 \mathrm{~m}$ & & Lead & 3onsst & 0.25 & & 3.5 & cllono & 13.85 & 624 & 1000 & so & $2.9 \times 10^{8}$ & $2.8 \times 10^{5}$ \\
\hline 85. 29 的 & & Lead & 304531 & 0.25 & 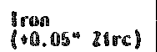 & 3.8 & CUno & 13.2 & 823 & 10.300 & 550 & $2.8 \times 10^{6}$ & $2.9 \times 10^{8}$ \\
\hline at $.30 \mathrm{~m}$ & & leads & sanssi & 0.25 & & 0.28 & Sand-y & 2.5 & s01 & 200 & 36 & 35 & $8.1 \times 100^{5}$ \\
\hline 甘1E. $31 \mathrm{M}$ & & Lead. & seasst T & 0.25 & trown & 0.25 & Sond-a & 10.5 & 364 & 800 & 35 & $2.9 \times 10^{\circ}$ & $2.9 \times 10^{5}$ \\
\hline 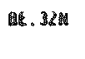 & & teas & Sanssi & 0.25 & (Ir) & 0.25 & Sand-घ & 2.35 & 499 & 2.2010 & 1.5000 & 15000 & $0.1 \times .10^{5}$ \\
\hline 86.3.3. & & Lead & JuAsST & 0.25 & 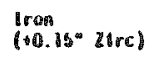 & 0.25 & Sandit-8 & 10.38 & 562 & 2.201013 & $1.5 \sin 3$ & $3.0 \times 80^{8}$ & $2.9 \times 10^{5}$ \\
\hline BE. 3896 & & Lead & suasst & 0.25 & Iroun & 3.5 & Sand $-\theta$ & 13.25 & 538 & 290 & iso & $2.9 \times 18^{8}$ & $2.9 \times 80^{5}$ \\
\hline Bt. 351 & & tead & suassit & 0.28 & 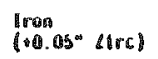 & 3.5 & Send-8 & 13.2 & 556 & 1.4000 & 650 & $2.9 \backsim 10^{4}$ & $2.9 \times 10^{5}$ \\
\hline 86. 366 & & lead & 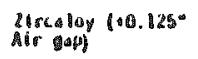 & 0.25 & Iron & 3.5 & Sond $-\theta$ & 13.25 & 357 & 2.7000 & 2.3000 & $3.1 \times 10^{4}$ & $2.9 \times 10^{5}$ \\
\hline В६. 3月M & & tead & $\begin{array}{l}\text { Lircaloy } 100.25^{\circ} \\
\text { Air gapy }\end{array}$ & c. 28 & Iron & 3.5 & Sand-B & 13.25 & 596 & 2.2400 & 2.5000 & $3.1: 30^{8}$ & $8.8 \times 10^{5}$ \\
\hline of sem & & Leas & 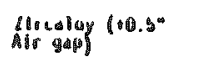 & 0.25 & Iron & 3.5 & Sands-e & 13.25 & 5s5 & 2,7000 & 3.5100 & $3.8 \times 10^{8}$ & $2.8 \times 10^{5}$ \\
\hline 85. .39: & & Leat & $\begin{array}{l}\text { Rarcslay (ol. } \\
\text { Air gap) }\end{array}$ & 0.25 & Iron & 3.5 & Sand-B & 13.25 & 552 & 2.700 & 8.5000 & $3.1 \times 10^{8}$ & $2.8 \times 10^{5}$ \\
\hline
\end{tabular}


Table 5-6. Concept BE Results in Current Study. (Continued)

\begin{tabular}{|c|c|c|c|c|c|c|c|c|c|c|c|c|c|}
\hline 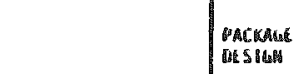 & MLASA & 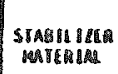 & 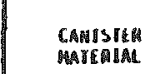 & 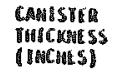 & $\begin{array}{l}\text { SEREVE } \\
\text { MATERAALA }\end{array}$ & 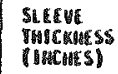 & 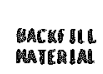 & 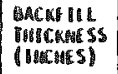 & win. was & $\begin{array}{l}\text { Lracto alg } \\
\text { Aworic }\end{array}$ & 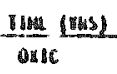 & 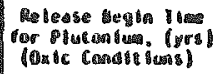 & 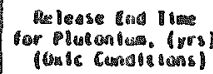 \\
\hline Ute. in & Easalt & Lead & 3005SI & 0.25 & Iron & 0.25 & Sand-it & 16.5 & 608 & 3, s00 & 8.6000 & $3.0 \times 10^{6}$ & $2.8 \times 10^{5}$ \\
\hline ot.611 & & looss & 300558 & 0.25 & fron & 0.25 & $\operatorname{sand}-8$ & 16.35 & 600 & 8.6000 & 3.0403 & $3.1 \times 10^{8}$ & $3.9=10^{5}$ \\
\hline 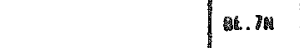 & & L.ead & 300sst & 0.25 & Iron & 6.0 & Sand-8 & 10.0 & seo & 5.500 & 3.900 & $3.2 \times 10^{8}$ & $2.9 \times 10^{5}$ \\
\hline Ac. & & lead & Zincaloy & 0.25 & & 0.25 & Sand-B & 16.5 & 602 & 3.300 & 3.300 & $6.0 \times 10^{8}$ & $2.8 \div 10^{5}$ \\
\hline Me. 1226 & & Leed & Iircaloy & 0.25 & Irom & 3.5 & Sand-a & 13.8 & 561 & 6.200 & 3.9400 & $3.4 \times 80^{4}$ & $2.8 \times 10^{8}$ \\
\hline 68.139 & & Lead & atratay & 0.25 & Irron & 0.25 & Sands-B & 16.35 & 600 & 8.4000 & 8,0000 & $3.3 \times 10^{8}$ & $2.9 \times 10^{8}$ \\
\hline 8k. $19 \mathrm{M}$ & & tead & Itrastay & 0.25 & Iran & 6.0 & Sand-18 & 10.8 & 580 & 5.5000 & 4.900 & $3.3 \times 10^{8}$ & $2.9 \times 10^{3}$ \\
\hline แt. Iss & & leats & Imonel & 0.25 & Iron & 0.25 & Sand-8 & 16.5 & 602 & 1930 & 1.3000 & $3.0 \times 10^{8}$ & $2.9 \times 80^{5}$ \\
\hline 6t. $80 \mathrm{M}$ & & leas & Inconel & 0.25 & 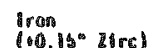 & 0.25 & Sand-B & 16.35 & 600 & 2.400 & $2_{0} 400$ & $3.1 \times 10^{\circ}$ & $2.8 \times 10^{5}$ \\
\hline 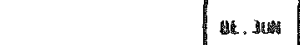 & & Lead & 3anssi & 0.25 & Iren & 0.25 & $\operatorname{san} u-\theta$ & 2.5 & Sod & 3,3000 & 1.6000 & 1.600 & $0.1 \times 10^{5}$ \\
\hline 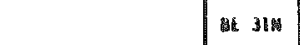 & & lead & massit & 0.25 & Iron & 0.25 & Sond-8 & 10.5 & 578 & 3. 5000 & 1.0000 & $3.0=10^{8}$ & $2.9 \times 10^{5}$ \\
\hline $86.32 \mathrm{~N}$ & & L.eoded & 804358 & 0.25 & Irow & ๑. 25 & $\operatorname{Sands}-B$ & 2.35 & 506 & 8.8000 & 3.1000 & 3.1000 & $4.1=10^{5}$ \\
\hline e日 3 3. & & lead & 3004ssir & 0.25 & trown & 0.25 & Sonst-a & 10.35 & 569 & 8,0000 & 3.1000 & $3.1 \div 10^{8}$ & $2.9 \times 10^{5}$ \\
\hline at $34 \mathrm{~N}$ & & bead & Sonsisl & 0.25 & Iren & 3.5 & Sand-B & 83.25 & 560 & 3.000 & 8.won & $3.0 \times 100^{6}$ & $2.8 \times 100^{5}$ \\
\hline Bt $25 \mathrm{H}$ & Shalle & leass & Eorcistoy & 0.25 & 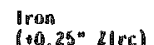 & 3.5 & Sand-B & 13.0 & 843 & 6.200 & 5.9000 & $3.4 \times 10^{6}$ & $2.8 \times 10^{3}$ \\
\hline 66.26" & & tead & massid & 0.25 & & 1,5 & Dene & 13.25 & 013 & 3.000 & 8.000 & $\cdots$ & $\cdots$ \\
\hline HE. $28 \mathrm{~m}$ & & tead & goosist & 0.26 & $\operatorname{lran}^{\prime \prime a n}$ & 3.5 & Bene & 03.2 & 612 & ه.2200 & 2.2013 & $\cdots$ & $\cdots$ \\
\hline 2ше & 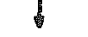 & teas & 30453 I & 0.25 & Inem & 3.5 & Clono & 13.25 & 603 & 3.70000 & 1.000 & $3.0 \mathrm{a} 10^{6}$ & $2.8 \times 10^{5}$ \\
\hline
\end{tabular}


Table 5-7. Effect of Backfill Material and Thickness on Maximum vaste Temperature.

\begin{tabular}{|c|c|c|c|c|}
\hline $\begin{array}{l}\text { Package } \\
\text { Design }\end{array}$ & Geology & Backfill Material & $\begin{array}{l}\text { Backfill } \\
\text { Thickness } \\
\text { (Inches) }\end{array}$ & $\begin{array}{l}\text { Peak Waste } \\
\text { Temperature, }\left(O_{K}\right)\end{array}$ \\
\hline BE. IN & Salt & Sand-Bentonite $(10 \%)$ & 16.5 & 595 \\
\hline BE. 3ON & & Sand-Bentonite $(10 \%)$ & $2 . \overline{5}$ & 501 \\
\hline$B E .31 N$ & & Sand-Bentonite $(10 \%)$ & 10.5 & 564 \\
\hline$B E .6 \mathrm{~N}$ & & Sand-Bentonite $(10 \%)$ & 16.35 & 593 \\
\hline$B E .32 N$ & & Sand-Bentonite $(10 \%)$ & 2.35 & 499 \\
\hline BE. 33N & & Sand-Bentonite $(10 \%)$ & 10.35 & 562 \\
\hline$B E .26 \mathrm{~N}$ & & Bentonite & 13.25 & 624 \\
\hline$B E .28 N$ & & Clinoptilolite & 13.25 & 624 \\
\hline$B E .34 N$ & & Sand-Bentonite $(10 \%)$ & 13.25 & 557 \\
\hline E. $2 \mathrm{~N}$ & & Sand-Bentonite $(10 \%)$ & 17.0 & 599 \\
\hline$E .15 N$ & & Sand-Bentonite (10\%) & 11.0 & 568 \\
\hline E.T7N & & Sand-Bentonite $(10 \%)$ & 3.0 & 505 \\
\hline E.19N & & Bentonite & 17.0 & 598 \\
\hline E.27N & 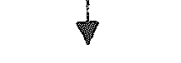 & Clinoptilolise & 17.0 & 698 \\
\hline
\end{tabular}


Sensitivity analyses were perfomed to determine the effects of certain package physical characteristics and geologic conditions on package perfomance. Previous sensitivity study results were reported in (Stula, 1980a) for evaluation of the list of best package concepts for salt and shale media as determined in FY'79 work (Lester, 1979). In the current study, the effects of variation of repository temperature and pressure, waste heat generation rate, gap thickness between package barriers, backfill thickness and compaction coefficients, and radionuclide solubility are evaluated.

The effects of variation of repository pressure on package perfomance are summarized in Table 5-8. Repository pressure was found to have no effect on designs utilizing a cast stabilizer. However, for non-cast stabilizer designs, canister thickness at failure and hence leach begin time are affected significantiy. As repository pressure increases, canister thickness required to withstand media creep forces increases and leach begin time, or time of canister failure, decreases.

The effects of variation of repository temperature on package perfomance are summarized in Table 5-9. In all cases, peak waste temperature is affected only to the extent that repository temperature varies. That is, the $\Delta T$ between repository and waste is constant and dependent on waste heat generation rate. Repository temperature was found to have a small, but significant effect on canister thickness at failure for the non-cast stabilizer designs. The criteria used to determine minimum canister thickness required to withstand geologic creep forces are temperature dependent. Thus, leach begin time is inversely related to canister thickness at failure. For cast stabilizer designs, no effects on canister thickness at failure or leach begin time are evident. According to the BARIER corrosion model, temperature would affect corrosion rate to the extent that one of two corrosion rates corresponding to two temperature ranges would be utilized in any particular corrosion calculation.

The effect of waste heat generation rate on the calculated maximum waste temperature for several package designs is shown in Table 5-10. It can be seen that for package designs 02.1 and $B E .27 \mathrm{~N}$, the maximum waste temperature increases linearly with increasing waste heat generation rate. This is to be expected from the nature of the heat transfer Equation (3.2.3) for heat transfer by conduction only. For heat transfer by conduction and radiation, use of Equations (3.2.3) - (3.2.5) with package design BE.39N shows essentially a linear 
Table 5-8. Effect of Repository Pressure on Package Performance.

\begin{tabular}{|c|c|c|c|c|c|c|}
\hline Package & Geology & $\begin{array}{l}\text { Repository } \\
\text { Pressure, (psi) }\end{array}$ & $\begin{array}{l}\text { Repository } \\
\text { Temperature, }\left({ }^{{ }} \mathrm{K}\right)\end{array}$ & $\begin{array}{l}\text { Can Thickness } \\
\text { at Failure, (in) }\end{array}$ & $\begin{array}{l}\text { Radiation } \\
\text { Dose, }(\mathrm{R} / \mathrm{hr})\end{array}$ & $\begin{array}{l}\text { Leach Begin } \\
\text { Time, (yrs) }\end{array}$ \\
\hline A. 10 & Salt & 1700 & 466 & .321 & .230 & 6790 \\
\hline A. 10 & & 2500 & 466 & .491 & .245 & 5090 \\
\hline A. 10 & & 3000 & 466 & .603 & .254 & 3970 \\
\hline$E .24$ & & 1700 & 466 & 0 & .089 & 2600 \\
\hline$E .24$ & & 2500 & 466 & 0 & .089 & 2600 \\
\hline E.24 & $\downarrow$ & 3300 & 466 & 0 & .089 & 2600 \\
\hline
\end{tabular}


Table 5-9. Effect of Repository Temperature on Package Performance.

\begin{tabular}{|c|c|c|c|c|c|}
\hline Package & Geology & $\begin{array}{l}\text { Repository } \\
\text { Temperature, }\left({ }^{\circ} \mathrm{K}\right)\end{array}$ & $\begin{array}{l}\text { Peak Waste } \\
\text { Temperature, }\left({ }^{\circ} \mathrm{K}\right)\end{array}$ & $\begin{array}{l}\text { Can Thickness } \\
\text { at Failure, (in) }\end{array}$ & $\begin{array}{l}\text { Leach Begin } \\
\text { Time, (yrs) }\end{array}$ \\
\hline A. 10 & Salt & 373 & 401 & .464 & 5360 \\
\hline A. 10 & & 423 & 451 & .478 & 5220 \\
\hline A. 10 & & 466 & 494 & .491 & 5090 \\
\hline A. 10 & & 523 & 551 & .509 & 4910 \\
\hline A. 10 & & 573 & 601 & .526 & 4740 \\
\hline E.24N & & 373 & 506 & 0 & 2600 \\
\hline E.24N & & 423 & 556 & 0 & 2600 \\
\hline E.24N & & 466 & 599 & 0 & 2600 \\
\hline E.24N & & 523 & 656 & 0 & 2600 \\
\hline E.24N & $\downarrow$ & 573 & 706 & 0 & 2600 \\
\hline
\end{tabular}


Table 5-10. Effect of Waste Heac Generation Rate on Maximum Waste Temperatura.

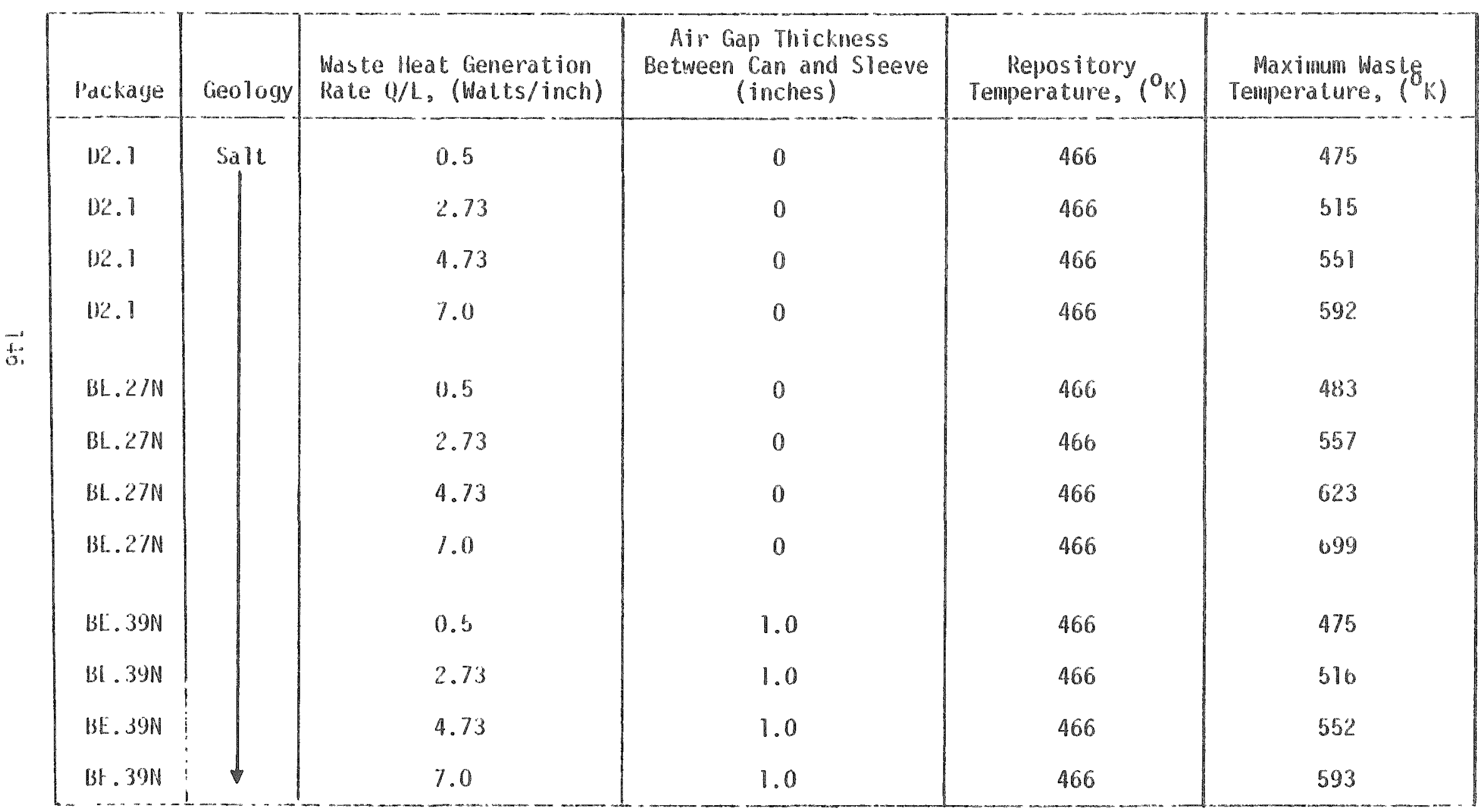


dependence of maximum waste temperature on waste heat generation rate. This indicates that for relatively small air gap thicknesses within a package, the radiation component of heat transfer is of minor importance in comparison to the conduction component. The effect of varying air gav thickness on naximum waste temperature is shown in Table 5-11 for sinall air gaps.

Variation of backfill compaction coefficients was found to have no effect on package life or any other perfomance characteristic with the exception of net pressure on a barrier at failure. However, this effect is relatively minor over the range of compaction coefficients considered. Net pressure of $a$ barrier at failure with a "stiff" backfill is generally on the order of 5 psi higher than that for a barrier with a "soft" bacxfill in non-cast stabilizer package designs. This effect is shown in Table 5-i2. For cast stabilizer designs, net pressure of a barpier at failure is independent of backfill compaction coefficients.

The effect of variation of backfill thickness on radionuclide transport resistance is shown in Tabie 5-13. It can be seen that most of the radionuclide transport resistance as calculated by the RELEAS subroutine is attributed to tne backfill thickness except in the situation where the backfill thickness is extremely small (less than one inch). Radionuclide relaase rates reach steady state more quickly as the backfill thickness is decreased. Letailed results of these sensitivity calculations are included in Appendix $F$.

The effects of solubility of $\mathrm{U}-238$ on radionuclide reiease rate ara evident in the results of each package design evaluated. For the high solubility case, the release rate reaches steady state more quickly and is significantly higher than in the low solubility case. 
Table 5-11. Effect of Air Gap Thickness on Maximum Waste Temperature.

\begin{tabular}{|c|c|c|c|c|c|}
\hline Package & Geology & $\begin{array}{l}\text { Waste Heat Generation } \\
\text { Rate }(\mathrm{L} / \text {, (Watts/inch) }\end{array}$ & $\begin{array}{c}\text { Air Gap Thickness } \\
\text { Between Can and Sleeve } \\
\text { (inches) }\end{array}$ & $\begin{array}{l}\text { Repository } \\
\text { Temperature, }\left({ }^{0} \mathrm{~K}\right)\end{array}$ & $\begin{array}{l}\text { Maximum Was te } \\
\text { Temperature, }\left({ }^{\circ} \mathrm{K}\right)\end{array}$ \\
\hline BE. 39N & Salt & 4.73 & 0.125 & 466 & 556.6 \\
\hline BE. $39 \mathrm{~N}$ & & 4.73 & 0.25 & 466 & 556.0 \\
\hline BE. 39N & & 4.73 & 0.5 & 466 & 554.8 \\
\hline BE. 39N & & 4.73 & 1.0 & 466 & 552.2 \\
\hline $\mathrm{B} 1.26 \mathrm{~N}$ & & 4.73 & 0.25 & 466 & 559.1 \\
\hline $\mathrm{B} 1.27 \mathrm{~N}$ & & 4.73 & 0.5 & 466 & 557.9 \\
\hline B $1.28 \mathrm{~N}$ & & 4.73 & 1.0 & 466 & 552.4 \\
\hline$B 1.29 \mathrm{~N}$ & $\downarrow$ & 4.73 & 2.0 & 466 & 547.7 \\
\hline
\end{tabular}


Table 5-12. Effect of Backfill Compaction Coefficients on Net Pressure on a Barrier at Failure.

\begin{tabular}{|c|c|c|c|c|c|}
\hline \multirow[b]{2}{*}{ Package } & \multirow[b]{2}{*}{ Medium } & \multirow[b]{2}{*}{ Barrier } & \multicolumn{2}{|c|}{ Backfill Compaction Coefficients } & \multirow{2}{*}{$\begin{array}{l}\text { Net Pressure on Barrier } \\
\text { at Failure, (PSIA) }\end{array}$} \\
\hline & & & A & k & \\
\hline B1. $4 \mathrm{~N}$ & Salt & Canister & 0.44 & 253 & -2522.6 \\
\hline B1. $19 \mathrm{~N}$ & & Canister & 47.5 & 0 & -2523.1 \\
\hline B1. $4 \mathrm{~N}$ & & Sleeve & 0.44 & 253 & -2869.1 \\
\hline B1. $19 \mathrm{~N}$ & & Sleeve & 47.5 & 0 & -2870.4 \\
\hline E.IN & & Cast Stabilizer & 0.44 & 253 & -2500 \\
\hline E.23N & & Cast Stabilizer & 47.5 & 0 & -2500 \\
\hline E.IN & & Canister & 0.44 & 253 & -2500 \\
\hline E.23N & $\downarrow$ & Canister & 47.5 & 0 & -2500 \\
\hline
\end{tabular}


Table 5-13. Effect of Backfil1 Thickness on Radionuclide Transport Resistance.

\begin{tabular}{|c|c|c|c|c|}
\hline \multirow[t]{11}{*}{$\begin{array}{l}\text { Package } \\
\text { Design }\end{array}$} & $\begin{array}{l}\text { Backfill* } \\
\text { Thickness, } \\
\text { (Inches) }\end{array}$ & $\begin{array}{l}\text { Radionuclide Transport } \\
\text { Resistance Due to } \\
\text { Backfill, }(\%)\end{array}$ & $\begin{array}{l}\text { Release Begin Time } \\
\text { for Plutonium, (yrs) } \\
\text { (oxic Conditions) }\end{array}$ & $\begin{array}{l}\text { Release End Time } \\
\text { for Plutonium, (yrs) } \\
\text { (Oxic Conditions) }\end{array}$ \\
\hline & 16.5 & 97.6 & $2.9 \times 10^{4}$ & $2.9 \times 10^{5}$ \\
\hline & 10.5 & 96.2 & $2.9 \times 10^{4}$ & $2.9 \times 10^{5}$ \\
\hline & 2.5 & 85.8 & 40 & $4.1 \times 10^{5}$ \\
\hline & 1.25 & 75.2 & 40 & $4.2 \times 10^{5}$ \\
\hline & 0.5 & 54.8 & 40 & $4.2 \times 10^{5}$ \\
\hline & 17.0 & 98.4 & $5.9 \times 10^{4}$ & $2.9 \times 10^{5}$ \\
\hline & 11.0 & 97.6 & $3.1 \times 10^{4}$ & $2.9 \times 10^{5}$ \\
\hline & 3.0 & 91.6 & 2500 & $3.9 \times 10^{5}$ \\
\hline & 1.5 & 84.5 & 2500 & $4.3 \times 10^{5}$ \\
\hline & 0.5 & 64.5 & 2500 & $4.2 \times 10^{5}$ \\
\hline
\end{tabular}

* Sand-Bentonite $(10 \%)$ 


\section{CONCLUSIONS AND RECOMMENDATIONS}

The objective of the System Study on Engineered Barriers (SSEB) was to evaluate the efficacy of engineered waste packages in reducing the potential dose to the population due to releases after repository closure. The information from the study will be used to $p$ lan development work on engineered barriers and assess the technical incentives for use of mul tiple barrier packages.

Only a limited number of engineered barrier package designs have been analyzed in the SSEB since the work was limited to scoping studies to guide future work. Many engineering designs can be proposed which have not been considered but the tools have been developed to analyze additional designs. The emphasis on future programs should not be on the "best" package but rather a "sufficient" package to meet necessary criteria. The BARIER code provides a means to measure proposed packages against such criteria.

\subsection{PACKAGE PERFORIAANCE}

On the basis of the post-closure, flooded repository scenario the preliminary analyses indicate that long-lived packages with low release rates can be designed. The performance model indicates that lifetimes of well over 1,000 years and in many cases over 10,000 years are reasonable to expect from packages constructed of common materials. Furthermore, judicious use of backfills to sorb radionuclides and/or exclude water can greatly reduce radionuclide releases after failure of canisters and overpacks as well as greatly delay the onset of radionuclide release.

The results indicate that a few inches of backfill tnickness are sufficient to supply the necessary barrier to radionuclide release. Radionuclide retention times for $U-238$ as long as $10^{7}$ years (e.g. Concept 8E.26iv) were calculated in many cases. Large backfill thicknesses are of littie advantage as long as sufficient sleeve thicknesses and/or a cast stabilizer are used. Thus a well chosen chemical sorbent would be a good choice with only a small amount: required. The stress defense contribution of the backfill is questionabie as it 
contributes very little and never contributes to stress application if a very "soft" material is used. The key question with regard to backfills remains whether the backfill material will retain its properties over long periods of time (more than 1,000 years). As was reported from previous studies (Lester, 1979 ) there is a serious question that a backfill would be intact in an environment capable of leaching material from a ceramic fuel material.

The new code version demonstrates, as did the previous version, that in creeping geologic media (salt or shale) the most important requirement is to withstand the media crushing pressure. This requires a heavy sleeve and/or a solid, "crush-proof" waste form (i.e., a cast stabilizer). Corrosion is important in that it steadily weakens a sleeve wall and eventually causes failure. In the case of the "crush-proof" stabilizer, corrosion results in a breakthrough which allows repository water to contact the waste.

In general, the results using the new BARIER model roughly correspond to those from the previous model. While corrosion rates in the new data. base tend to be higher than the previous values, the stress calculations for crushing forces are based on real failure rather than ASME code criteria which tend to be very conservative.

\subsection{AREAS OF UHCERTAINTY}

The current performance calculation capability is quite comprehensive but still contains areas of uncertainty. Most of the concern is the lack of sufficient data in appropriate environments needed to support more sophisticated approaches. Generally, where such uncertainty exists, credit is not taken for possible lesser consequences. For instance, when a particular parameter value is not well known, bounding values are used and the limit yielding the worst consequences in the bounded range is chosen. Where the dependency of an effect on a certain parameter is not well understood the parameter is set constant at the limiting value yielding the worst consequences. 
6.2.1 Corrosion Rate

Corrosion of package barriers is a dominating fallure mechanism. To accurately assess the life of the package the corrosion rate as a function of key parameters and of time is needed. Extensive study of literature supported by hand and computer-assisted searches was performed to develop the best data base possible. The goal was to incorporate various modes of corrosion such as crack propagation, pitting, graphitization and bulk corrosion and to include other parameter effects such as temperature and radiaiton. Three deficiencies were encountered: (1) total lack of data in some categories for some materials, (2) data available but in chemical environments not corresponding to the repository condition of interest, and/or (3) ranges of parameters (temperature, pressure) not corresponding to those of interest. It was possible to divide corrosion rates into two temperature ranges and choose high values in known data ranges. One difficulty was encountered in choosing "highest values"; unreasonable rates sometimes result which lack common sense. In most of these instances the environment was too different from the repository. Such values were deleted.

The effect of radiation on corrosion is not well documented. Data that are available indicate a small effect for exposures of interest. A review of the detailed results shows that the radiation fieids are generally very low at failure time compared to levels giving measureable effect. However, more information, especially in typical chemistry, is definitely needed or much overdesign will be required.

Another area of particular concern is local corrosion on joints, seams or other discontinuities in the bulk metal. Corrosion data is typically on base metal samples al though some weldment testing has been done. Nevertheless, it is possible to introduce safety factors. For example, the literature gives zuidance on corrosion allowances for non-base metals and one can design in conservation.

In general it is felt that package designs could proceed now if a large degree of overdesign is tolerable. To reduce cost and increase general confidence more pertinent corrosion data would be useful. Of special concern is the need to extrapolate over long periods of time. This is unavoidable and can be done with more confidence if based on comprehensive data. 


\subsubsection{Backfill Properties}

Two basic categories of backfill properties are used in the current BARIER code: (1) Physicomechanical and (2) chemical. The physico- mechanical properties are volume-pressure compaction characteristics (bulk modulus) and internal friction characteristics (shear modulus and Mohr slope) which determine the behavior of the backfill under external stress and how the repository stress is transmitted to the inner package components. The chemical charateristics are those parameters affecting radionuclide and water cransport and include retardation factors $\left(k_{d}\right)$, diffusivity, porosity and tortuosity.

Data are limited in both categories but reasonable sounding estinates can be made and are used in the current study. As with corrosion, increasing knowledge allows more precision in design. Confidence in the design is possible with current data but thick backfilis may be needed to ensure sufficient perfomance. The greatest uncertainty is the ability of backfills to retain their properties over long periods in envrionments of interest. However. geologic data on montmorillonites and similar materials provide some insignt in their stability thus allowing the bounding process. The most pessimistic conclusion leads to significant functional lifetimes (thousands of years) so that the problem is again one of design precision rather than of design integrity.

The vital importance of the backfill is evident in the results of tnis study. Therefore, it would be useful to expand understanding of these materials as a design suppopt activity. increased confidence and design precision in the backfill will yield many benefits.

\section{2 .3 Waste Leaching}

This study has been restricted to disposal of unreprocessed, spent fuel. Nevertheless, all that is discussed here applies to any other waste forn.

Consider the relationship of intrinsic (microscopic) lata to zlobal (physical system) data. The package is a global system the descriotion of which is based on intrusion data. Data obtained to date is intrinsic (katayana, 1376, 1980); that is, the measurements of leach rate are taken witnout the resistance due to contaminants present. If the intrusion leach rate is large compared to global system transport then the leachate around the waste form becomes saturated with a given species. In this case solubility data aro needed. Solubility tata 
from Katayana were used because in all cases the transport rates were orders of magnitude below intrusion pates.

The release pates are sensitive to solubility so that more solubility data are needed depending on the desire to pinpoint release pates and breakthrough times. The extremely long times obtained raise some question as to whether one is concerned whether breakthrough times are $10^{3}$ years or $10^{10}$ years. However, as more detailed precision package design is done and more refined risk analysis is carried out, increased confidence in the pertinent numbers will be needed. It is recomended that solubility numbers be emphasized in future testing. Leach rate measurements should be done in demonstration testing with deliberately failed packages so that global rate modeling can be validatad.

\section{$6.2 .4 \quad$ other Areas of uncertainty}

Some factors are not accounted for in the BARIER nodel. Of note are water exclusion effects and the role of protective coatings (not including metal cladding). These could be considered as delay times to be added to the package performance times peported. However, the magnitude of these times remains relatively uncertain. iluch data are available on the behavior of swelling clays and protective coatings but not as a function of long periods of time. work in this area would be beneficial in providing a neasure of redundancy to design.

\subsection{RISK MODEL DEVELOPMENT RECOMENDATION}

The BARIER code was not developed for the purpose of risk analysis but rather to provide scoping studies which could be used to make research and development decisions. However, in recognition of the amount of effort expended, the code was designed with ease of extention to a risk model in nind. Thus the code provides a baseline for development of a gear-field risk model. 


\subsubsection{Applications of BARIER to Risk Analysis}

The current BARIER code closely resembles the consequence part of a risk model concerned with long-term effects in the post-closure repository. It is based on one principal scenario but many sub-scenarios could be evaluated from the results (see discussions in Section-2). It carries this principal scenario to the consequence conciusion: release to the geology of specific radionuclides as a function of time. What it lacks is sufficient data base and in some cases analytical features required for full risk analysis. It is not a probabilistic model but is fast running and so could be driven by a probabilistic model. Some improvements can be made to BARIER now with additional work and some improvements must await further data acquistition.

The sections that follow give some suggestions on changes to convert the model to a near-field risk analysis. Many other changes will likely be identified as the actual job of incorporating this model into risk methodology is undertaken.

\subsubsection{Near-Term Improvements}

Further improvenents in the release model could be made as discussed in Section 3.5. This includes adding the tail-off portion of the release curve. The benefits should be weighed against the added computational burden but can only be assessed by making the changes. Because of long delay times in the backfill, daughter product transport analysis capability would also be desirable. Analytical solutions can probably be developed for decay chains in the backfill with the flux boundary conditions. These would be similar to the GETOUT model but the boundary effects are different. More accurate representation of radiodecay in the backfill may be desirable for shorter-lived radionuclides.

The model for resistance effects of failed barriers could be coupled to corrosion mechanisms to allow a more accurate representation of the barrier resistance in radionuclide release. However, note that in inany cases this represents less than 10 per cent of the total radionuclide release resistance.

code function changes such as automatic variance of time increment to accommodate fast or slow rates of package degradation and to allow more efficient use of computational time would be desirable. In addition, further refinement of data manipulation and output format more appropriately tailored to interfacing in a risk model would be desirable. 
Expansion of the data base will be needed to include more construction materials and backfill materials. Other waste forms will also be needed for future risk studies. The current data base was limited for scoping purposes and was not meant to cover all the design choices which will be considered as package development proceeds. Corrosion rates, material stress properties, retardation factors, porosities, tortuosities, thermal properties, heat generation rates, and many other such data are needed for additional materials.

The expansion to include many other radionuclides would also be desirable for risk studies. However, some parameters such as retardation factors will be very data-limited.

\section{3 .3}

Long-Term Improvements

changes in this category are those which require new data for support. The most significant improvement would be the use of more sophisticated corrosion rate models with more confidence in extrapolation of rates and consideration of non-linear effects. In addition, feedback between crack propagation/penetration and calculated stresses might be a desirable feature. Of particular importance is the improvement of the data base so that more applicable chemical-physical environments are represented and more accurate temperature dependency is available. It is not likely that this can be completed from existing literature but a start could be made. The effect of backfill corrosion should be included. Chemical adjustment, corrosion agent transport in a backfill and water transport are all factors not now considered. Intrusion corrosion rates are employed to assess the global system. This results in higher corrosion rates than might actually be observed.

As data on nuclear radiation effects become available the feedback loop betiween corrosion and radiation field calculations could be closed. When this is done the radiation field subroutine will require expansion to include photon sources other than spent fuel (such a change might be desirable now in the near-term).

The current model envisions a lithostatic pressure acting on the package. In most creeping media the effect of the slope of excavation, discontinuities in the geology and other asymetric properties results in non-uniform stresses. Cross asymmetry may actually shorten package life. It would be desirable to do more sophisticated stress calculations. Furthermore, 
the conservative assumption is now made that full overburden stress is applied at time $=0$. At high temperature in salt this is quite reasonabie as creep rates are high at low temperature. In other media (e.g., shale) consideration of rate of stress build-up could be a useful addition. 


\section{REFERENCES}

American Society of Mechanical Engineers, 1977. Asme Boiler and Pressure Vessel Code, Section VIII, Division 1, JuTy.

Ah7strom, P. E., 1978. "Ceramic and Pure-Metal Canister in Buffer Material", Proceedings of the Conference on High-Level Radioactive Solid Waste Forms, NUREG/CP-0005, L. S. Casey, Ed., December.

Berry, W. E., 1971. Corrosion in Nuclear Applications, John Wiley \& Sons.

Bird, R. B., et a1, 1965. Transport Phenomena, John Wiley \& Sons.

Blazer, R. V., and J. J. Owens, 1956. "Special Corrosion Study of Carbon and Low Alloy Steels", ASTM Symposium on High Purity water Corrosion.

Braithwaite, J. W., and M. A. Molecke, 1979. Scientific Basis for Nuclear Waste Management and Technology, Vol. 1, G. 1. McCarthy, ed.s Plenum Press.

Bulischeck, T. S., and D. Van Rooyen, 1980. "Stress Corrosion Cracking of Alloy Goo Using Constant Strain Rate Test", NACE Corrosion/80, Preprint of Paper No. 183, March, 1980.

Butler, G., and H.C.K. Ison, 1966. Corrosion and Its Prevention in Water, Reinhold Publishing Co., New York.

Byerlee, J. D., 1967. "Frictional Characteristics of Granite Under High Confining Pressure", J. Geophys. Res., Vol. 72, No. 14, July 15.

Carslaw, H. W., and J. C. Jaeger, 1967. Conduction of Heat in solits, 0xford University Press.

Cataldi, H. A., and C. F. Cheng, 1958. "Investigation of Erosion and Corrosion of Turbine Materials in Wet Oxygenated Steam", Trans. ASME, Vol. 80. 
Cheng, C. F., 1980. "Canister Corrosion of a system Study on Engineered Barriers", Internal Letter", Science Applications, Inc.

Cooper, A. R. and L. E. Eaton, 1962. "Compaction Behavior of Several Ceramic Powders", J. Am. Ceram. Soc., Vol. 45, No. 3, March 1.

Copson, H. R., and W. E. Berry, 1960. "Qualifications of Inconel for Nuclear Power Plant Applications", Corrosion, Vol. 16, No. 1.

Danckwerts, P.V., 1951. Transactions of Faraday Society, Vol. 47, p. 1014.

Datsko, J., 1966. Material Properties and Manufacturing Processes, J. Wiley \& Sons, Inc., New York.

Dennison, I. A., and M. Romanoff, 1946. "Soil Corrosion Studies: Ferrous Metal and Alloys", J. Research, NBS, Vol. 44.

Ende11, et a1, 1938. "Uber Zusammenhange Zwischen Wasserhaushait der tomminerale and Boden physikalischen Eigenschaften bindiger Boden", Veroffent7. deut. Forsch. Bodenmech, 5.

Ford, F. P., and M. J. Povich, 1979. "The Effect of Oxygen Temperature Combinations of Stress Corrosion Crack Susceptibility of Sensitized Type 304 Stainless Steel in High Temperature Water", Corrosion, Vol. 35, No. 12.

Gee1, J., et a1, 1979. "Incorporation of Solid High Level Waste into Metal and Non-Metal Matrices", Proceedings of the Conference on High-Level Radioactive Solid Waste Forms, NUREG/CP-0005, L. A. Casey, ed., December.

Haggblom, H., 1977. Diffusion of Soluble Materials in a Fluid Filling a Porous Medium, KBS Technical Report 9, Stockholm, Sweden.

Hammer, N. E. Corrosion Data Survey, 5th ed., NACE, Houston, Texas.

International Nickel Co., Inc. General Seawater Corrosion, GM 3-785719 (2).

Katayama, Y. B., 1976. Leaching of Irradiated LWR Fuel Pellets in Deionized and Typical Ground Water, BNWL-2057, JuTy. 
Katayama, Y. B., 1980. Status Report on LWR Spent Fuel IAEA Leach Tests, PNL3173, Battelle Pacific Northwest Laboratories, March.

Laque, F. L., and H. R. Copson. Corrosion Resistance of Metals and Alloys, 2nd ed., Reinhold Publishing Co.

Lester, D. H., et a1, 1979. System Study on Engineered Barriers Task 3 Barrier Performance Analysis, Final Report, SAI01379-990LJ, Science Applications, Inc.s prepared for office of Nuclear waste Isolation, September.

Low, P. F., and J. F. Margheim, 1979. "The Swelling of Clay. I. Basic Concepts and Empirical Equations", Soil Sci. Soc. Amer. Jour., Vol. 43, pp. 473-481.

Marks, L. S., 1952. Mechanical Engineers' Handbook, 5th ed., McGraw-Hill Publishing $\mathrm{Co}$.

Mattson, E., and E. Fredrickson, 1968. "Pitting Corrosion in Copper Tubes: Causes of Corrosion and Countermeasures," British Con. J., Vol. 3, No. 5, September.

Ne7son, G. A., 1967. Corrosion Data Survey, NACE, Houston, Texas.

Neretnieks, I., 1978. Transport of Oxidants and Radionuclides Through a Clay Barrier, KBS Teknisk Rapport, February 20.

Posey, F., and A. A. Palko, 1979. Corrosivity of Carbon Steel in Concentrated Chloride Solution", Corrosion, Vol. 35, No. 1, January.

Powe 11, B. E., and L. V. Lucey, 1966. Physical Studies of Water Formed Corrosion Products on Copper, Final Report to the International Copper Research Assoc., British Non-Ferrous Metals Tech. Center, London, England, March.

Pusch, R., 1978a. Highly Compacted Na Bentonite as Buffer Substance, KBS Report 174, Stockholm, Sweden.

Pusch, R., 1978b. Self-Ingestion of Highly Compacted Bentonite into Rock Joints, KBS Report 173, Stockholm, Sweden. 


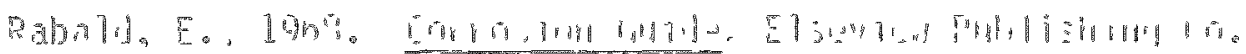

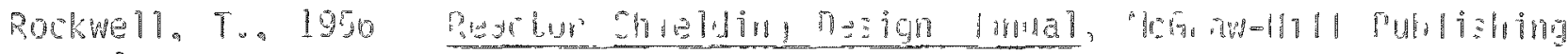
$\ln$

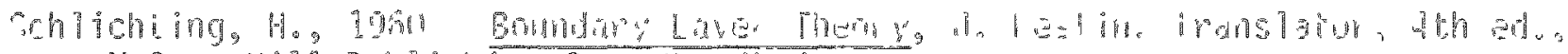

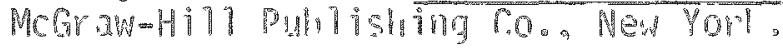

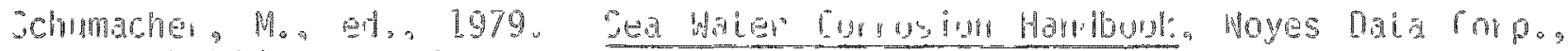
Parl Ridure, Nen yersey.

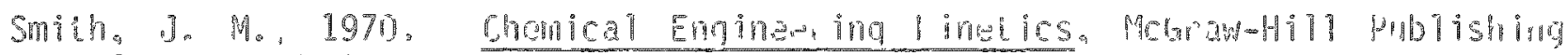
Co. New York.

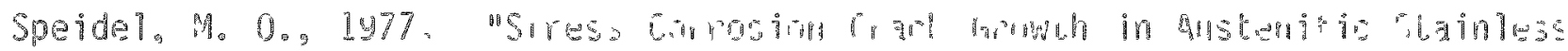
$5 \cos 7 \%$ Corrosian, $407,35,140.5$

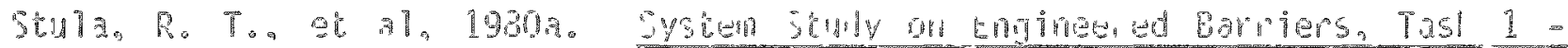
Interin Report, SAI0137y-107nLd/F, scien.e Apptications, Ince prepared

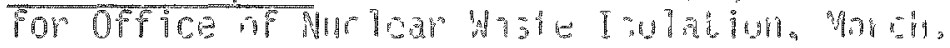

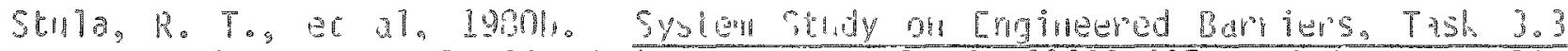

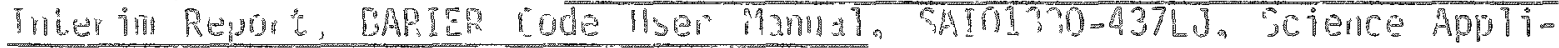

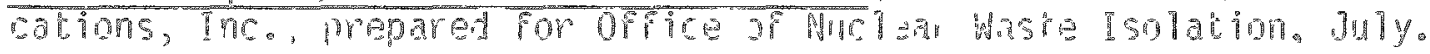

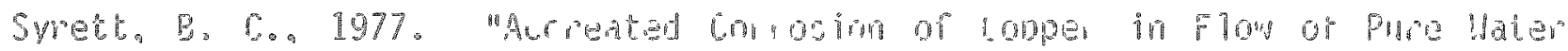

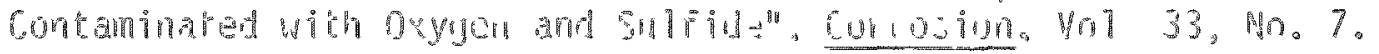

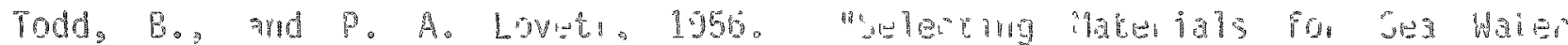

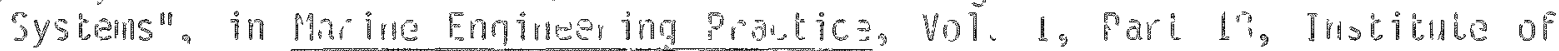
Marine Engineris. England.

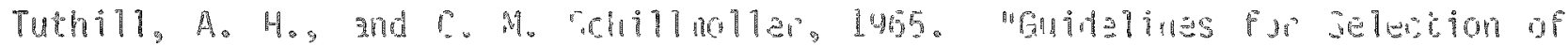

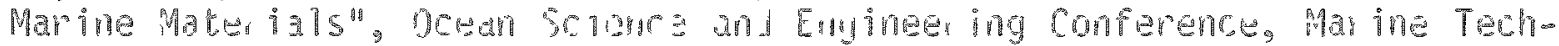

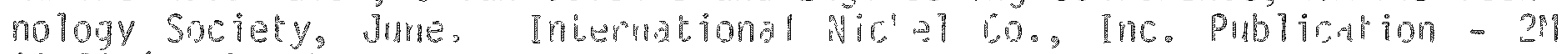
$11-76(592-76) 5303$

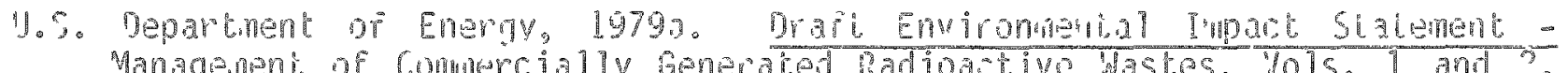
DoE/EIS-Dig6-D. Aprit. 
U.S. Departhent of Enargy, 1079h. Techrology for Commorcial Radioactive Waste

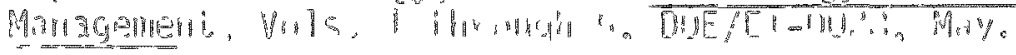

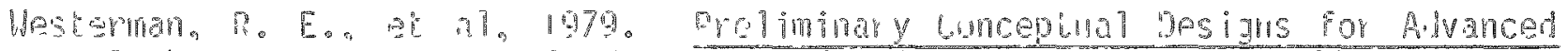

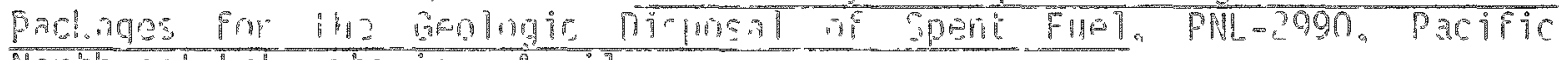

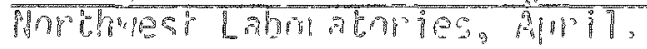


-

164

○ 


\section{NOMENCLATURE}

\begin{tabular}{|c|c|c|c|c|}
\hline SYMBOL & $\begin{array}{l}\text { PROGRAM } \\
\text { VARIABLE }\end{array}$ & EQUATION & UNITS & EXPLANATION \\
\hline$A_{1}, A_{2}$ & $-\cdots$ & 3.6 .3 & Dimensionless & $\begin{array}{l}\text { Coefficients used in caluculation of bulldup } 1 \ldots: \cdots \text { in } \\
\text { RADCLC subroutine }\end{array}$ \\
\hline$A_{n}$ & AN & 3.5 .19 & $\mathrm{gm} / \mathrm{mm}$ & $\begin{array}{l}\text { Parameter in } n \text {-th term of Fourier series in MELES sub- } \\
\text { routine }\end{array}$ \\
\hline A & A & 3.4 .4 & Ksi & $\begin{array}{l}\text { Empirical coefficient used in backfill pressure-vessel } \\
\text { relationship in STRESS subroutine }\end{array}$ \\
\hline B & B & 3.6 .1 & Dimensionless & Buildup factor in flux calculation in RAOCLC subrouting \\
\hline B & BULK & 3.4 .13 & Ksi & Bulk modulus of Barrier wall in STRESS subroutine \\
\hline$B^{\prime}$ & BBULK & 3.4 .7 & Ksi & $\begin{array}{l}\text { Instantaneous bulk modulus of backfill in STRESS sub- } \\
\text { routine }\end{array}$ \\
\hline$B_{n}$ & $-\ldots$ & 3.5 .28 & $g m / m 1$ & $\begin{array}{l}\text { Parameter in } n \text {-th term of Fourier series in RELEAS sub- } \\
\text { routine }\end{array}$ \\
\hline$B_{1}, B_{2,}$ & $B 1, B 2$ & 3.4 .13 & Ksi & $\begin{array}{l}\text { Coefficients in bulk modulus equation for barrier wall } \\
\text { in STRESS subroutine }\end{array}$ \\
\hline$c_{0}$ & -- & 3.4 .1 & Ksi & Cohesion force in yield model of STRESS subroutine \\
\hline D & DFL & 3.5 .6 & $\mathrm{~cm}^{2} / \mathrm{yr}$ & $\begin{array}{l}\text { Diffusion coefficient in species of interest in liquid } \\
\text { in RELEAS subroutine }\end{array}$ \\
\hline$D_{e}$ & $-\cdots$ & 3.5 .5 & $\mathrm{~cm}^{2} / \mathrm{yr}$ & Eftective diffusion coefficient for porous medium \\
\hline E & E & 3.4 .44 & Dimensionless & $\begin{array}{l}\text { Joint efficiency for longitudinal sean in loop stress } \\
\text { calculation in STRESS subroutine }\end{array}$ \\
\hline$r\left(1, b_{2}\right)$ & H Func: & 3.6 .1 & Dimensionless & Function used in flux calculation in RADCLC subroutine \\
\hline G & SHEAR & 3.4 .14 & Ksi & Shear modulus of harrier wall in STRESS subroutine \\
\hline $\mathrm{G}_{1}, \mathrm{~B}_{\mathrm{g}}$ & $\mathrm{G1}, \mathrm{G} 2$ & 3.4 .14 & Ksi & $\begin{array}{l}\text { Coefficients in shear modulus equation for barrier wall } \\
\text { in STRESS subroutine }\end{array}$ \\
\hline$a^{\circ}$ & BSIIEAR & 3.4 .8 & Ksi & $\begin{array}{l}\text { Instantaneous shear modulus of backfill in STRESS sub- } \\
\text { routine }\end{array}$ \\
\hline$\|$ & $-\cdots$ & 3.5 .13 & $\mathrm{col}^{3} / \mathrm{yr}$ & Mass conductance at $x=\ell$ in RELEAS subroutine \\
\hline
\end{tabular}




\begin{tabular}{|c|c|c|c|c|}
\hline SYMBOL & $\begin{array}{l}\text { PROCRAM } \\
\text { VARIABLE }\end{array}$ & EUUATION & UNITS & EXPLANATION \\
\hline$H_{x}^{0}$ & HLCA & 3.5 .28 & $\mathrm{~cm}^{3} / \mathrm{yr}$ & $\begin{array}{l}\text { Mass conductance at } x=\ell \text { times area available for trans- } \\
\text { port in RELEAS subroutine }\end{array}$ \\
\hline I & I & $-\cdots$ & Dimensionless & $\begin{array}{l}\text { Barrier identification number for any package design } \\
\text { case (1-1 for innermost barrier) }\end{array}$ \\
\hline R & $-\cdots$ & 3.5 .29 & $\mathrm{gm} / \mathrm{yr}^{\mathrm{r}}$ & Parameter in Equation (3.5.29) of RELEAS subroutine \\
\hline K & K & 3.4 .4 & Ksi & $\begin{array}{l}\text { Empirical coefficient used in backfill pressure-volume } \\
\text { relationship in STRESS subroutine }\end{array}$ \\
\hline$k_{\ell}$ & AKL. & 3.5 .28 & $g m / y r$ & $\begin{array}{l}H_{l}^{0} \text { multiplied by concentration difference across the } \\
\text { resistance at } x=\ell \text { in RELEAS subroutine }\end{array}$ \\
\hline $\mathbb{L}$ &.- & 3.2 .2 & Inches & Length of Waste heat generation surface (cylindrical) \\
\hline$N_{d}$ & $-\cdots$ & 3.5 .5 & $\mathrm{gm} / \mathrm{cm}^{2}-\mathrm{yr}$ & $\begin{array}{l}\text { Flux of species of interest due to diffusion in RELEAS } \\
\text { subroutine }\end{array}$ \\
\hline p & $-\cdots$ & --- & Ksi & Pressure on a lransverse cylinder in Figure $2-3$ \\
\hline$p_{0}^{0}$ & -- & 3.4 .31 & Ksi & $\begin{array}{l}\text { Initial internal pressure on a barrier in STRESS sub- } \\
\text { routine }\end{array}$ \\
\hline$p_{1}^{0}$ & $-\cdots$ & 3.4 .32 & Ksi & Initial pressure at $R_{1}$ in STRESS subroutine \\
\hline p & $p$ & 3.2 .6 & Watts/in & $\begin{array}{l}\text { Variable used in PKTEMP subroutine for maximum wasle } \\
\text { temperature calculation }\end{array}$ \\
\hline$P_{R}$ & REPRES & 3.4 .1 & Ksi & $\begin{array}{l}\text { Absolute value of mean stress in yield model of STRESS } \\
\text { subroutine (repository pressure) }\end{array}$ \\
\hline$P_{1}$ & HWESS & 3.4 .29 & ksi & Pressure at $k_{1}$ in STRESS subroutine \\
\hline$P_{2}$ & -- & 3.4 .36 & Ksi & Pressure at $R_{2}$ in STRESS subroutine \\
\hline$P_{3}$ & REPRES & 3.4 .36 & Ksi & Pressure at $R_{3}$ in STRESS subroutine \\
\hline$P_{0}$ & $-\cdots$ & 3.4 .29 & psi & Intermal pressure on a barrier \\
\hline Prore & $\cdots$ & 3.4 .1 & ksi & Pore water pressure in yield model of STRESS subroutine \\
\hline Q & HIAT(G/L) & 3.2 .3 & Watls & Radial waste heat generation of packaged waste \\
\hline ! $\mathrm{i}$ & $-\cdots$ &.-- & gmin & Initial radionuclide quantities \\
\hline
\end{tabular}




\begin{tabular}{|c|c|c|c|c|}
\hline SYMBOL & $\begin{array}{l}\text { PROGIRAM } \\
\text { VARIABLE }\end{array}$ & EOUATION & DNITS & EKPLANATION \\
\hline$R$ & $-\infty$ & 3.4 .23 & Inches & Dulside radium of cylinder in STRESS subroutine \\
\hline$R_{e}$ & $-\cdots$ & $-\cdots$ & Dimensionless & Reynolds number \\
\hline $\mathbb{R}_{f}$ & $-\infty$ & 3.5 .43 & Dimensiontess & Retard factor in RELEAS subroutine \\
\hline $\mathbb{R}_{0}$ & IOIAM & 3.4 .29 & Inches & $\begin{array}{l}\text { Radius of inner surface of innermost barrier laver in } \\
\text { STRESS subroutine }\end{array}$ \\
\hline$R_{1}$ & ODMM & 3.4 .29 & Inches & $\begin{array}{l}\text { Radius af outer surface of fmutrmosc barrich layer in } \\
\text { STRESS subroutine }\end{array}$ \\
\hline$R_{2}$ & (DDMAM: & 3.4 .06 & Inches & Radius of outer surface of second innermosi barmier ta: er \\
\hline$k_{3}$ & FDIAM & 3.4 .36 & Inclies & Rarlius of outer surface of filler ur backitil \\
\hline$R_{0}$ & (1) & 5.60 .1 & Cly & Raúliss of waste assemble \\
\hline$R_{C}$ & CORRAT & 3.3 .1 & $\operatorname{im} / y r^{\circ}$ & Conposion rates \\
\hline$\ddot{R}$ & $-\cdots$ & 1.4 .83 & $\mathrm{in} / \mathrm{sec}^{2}$ & $d^{2} R / d t^{2}$ \\
\hline$s$ & PMAXW & 3.4 .44 & psi & $\begin{array}{l}\text { Alowable stress for waterial in hou siress calculation } \\
\text { in sTRESS subrutine }\end{array}$ \\
\hline$\$ 1,54$ & 81,91 & 3.4 .85 & psi & $\begin{array}{l}\text { Constants in equations to calculate allowable stress in } \\
\text { stress subroutine }\end{array}$ \\
\hline$S$ & 52 & 3.4 .45 & $115 i / 00$ & Sabre as abrove \\
\hline 53 & $\$ 3$ & 3.4 .45 & "C & Sume als above \\
\hline$S_{V}$ & sV & 3.6 .1 & Photons/cin $/ \mathrm{sec}$ & $\begin{array}{l}\text { Source intensity (cylindrical) in flux calculation in } \\
\text { RALCLC subrouline }\end{array}$ \\
\hline$T_{0}$ & $-\infty$ & 3.5 .66 & yr & Time at which zero inventory is obtained in the waste \\
\hline$T$ & TCENT & 3.4 .12 & "r: & Tenperature of barrier wall in :TRESS subroutine \\
\hline$I_{A}$ & $\begin{array}{l}\text { Maximp (for in- } \\
\text { hermost barrien } \\
\text { layer only) }\end{array}$ & 3.2 .3 & OK & $\begin{array}{l}\text { Temperature at inner surfact of barrier layer (= MAXTMP } \\
\text { for innermost barrier) }\end{array}$ \\
\hline$T_{B}$ & TOUTER & 3.2 .4 & OK & $\begin{array}{l}\text { Temperature at culer surface of nutermost (gas gan) } \\
\text { barrier layer }(=\text { cemperature of geology for outermost }\end{array}$ \\
\hline
\end{tabular}




\begin{tabular}{|c|c|c|c|c|}
\hline SYMBOL & $\begin{array}{l}\text { PROGRAM } \\
\text { VARIABLE }\end{array}$ & EQUATION & UNITS & EXPLANATION \\
\hline $\mathrm{T}_{3}$ & TINNER & 3.2 .3 & $o_{K}$ & Temperature at outer surface of filler or backfill \\
\hline$T_{R}$ & REPTEM & 3.2 .1 & OK & Temperature at repository surface \\
\hline $\mathrm{T1}, \mathrm{T4}$ & $T 1, T 4$ & 3.2 .1 & ${ }^{\circ} \mathrm{C}$ & Constants used in repository temperture calculation \\
\hline T2 & $\mathrm{T} 2$ & 3.2 .1 & ${ }^{\mathrm{o}} \mathrm{C} / \mathrm{yr}$ & Same as above \\
\hline$T 3$ & T3 & 3.2 .1 & yr & Same as above \\
\hline$T_{c}$ & TEMP & 3.2 .7 & $o_{K}$ & $\begin{array}{l}\text { Temperature at outer surface of barrier layer (TEMPER } \\
\text { subroutine) }\end{array}$ \\
\hline$U^{\prime}$ & --- & 3.4 .36 & Inches & Radial displacement at $R_{2}$ in STRESS subroutine \\
\hline$u$ & -- & 3.4 .25 & Inches & Radial displacement - STRESS subroutine \\
\hline v & $-\ldots$ & -- & $\mathrm{in} / \mathrm{sec}$ & Fluid velocity around a transverse cylinder in Figure 2-3 \\
\hline V & $-\cdots$ & 3.4 .3 & & Backfill volume after compression by pressure $P_{2}$ \\
\hline$v_{0}$ & $-\cdots$ & 3.4 .3 & $\mathrm{~cm}^{3}$ & Original backfill volume \\
\hline$v^{*}$ & VSTAR & 3.4 .3 & Dimensionless & Indication of pressure-volume relationship of backfill \\
\hline$x_{n}$ & -- & 3.5 .19 & Dimensionless & $\begin{array}{l}\text { Parameter in } n \text {-th term of Fourier series in RELEAS sub- } \\
\text { routine }\end{array}$ \\
\hline$\gamma$ & Yield & 3.4 .12 & Ksi & Yield strength in yield model of STRESS subroutine \\
\hline$\gamma_{1}, \gamma_{2}$ & $y_{1}, y_{2}$ & 3.4 .12 & Ksi & $\begin{array}{l}\text { Coefficients in yield stress equation for barrier wall } \\
\text { in STRESS subroutine }\end{array}$ \\
\hline$a$ & -- & 3.4 .27 & Dimensionless & Constant in strain equations in STRESS subroutine \\
\hline a & -- & 3.6 .1 & $\mathrm{~cm}$ & $\begin{array}{l}\text { Distance to the point of interest from the edge of the } \\
\text { waste cylinder in the RADCLC subroutine }\end{array}$ \\
\hline$a_{1}, d_{2}$ & -- & 3.6 .3 & Dimensionless & $\begin{array}{l}\text { Coefficients used in calculation of buildup factor in } \\
\text { RADCLC subroutine }\end{array}$ \\
\hline b & $\cdots$ & 3.4 .27 & Dimerisiontess & Constant in strain uations in STRESS subroutine \\
\hline$b_{2}$ & -- & 3.6 .1 & Dimensionless & $\begin{array}{l}\text { Number of mean free paths to point of interest in the } \\
\text { RACLC subroutine }\end{array}$ \\
\hline
\end{tabular}




\begin{tabular}{|c|c|c|c|c|}
\hline SYMBOL & $\begin{array}{l}\text { PROGRAM } \\
\text { VARIABLE }\end{array}$ & EQUATION & UNITS & EXPLANATION \\
\hline b & $-\cdots$ & 3.5 .33 & Dimensionless & Radio-diffusion parameter in RELEAS subroutine \\
\hline$c_{i s}$ & $-\cdots$ & 3.5 .27 & $9 m / m l$ & $\begin{array}{l}\text { Concentration of species of interest in liquid at } \\
\text { infinite distance in a slab in RELEAS subroutine }\end{array}$ \\
\hline$c_{\ell}$ & $\cdots$ & 3.5 .2 & $\mathrm{gm} / \mathrm{ml}$ & $\begin{array}{l}\text { Concentration at } \ell \text { of species of interest in liquid in } \\
\text { RELEAS subroutine }\end{array}$ \\
\hline c & CONC & 3.5 .1 & $g m / m l$ & $\begin{array}{l}\text { Concentration of species of interest in Tiquid in RELEAS } \\
\text { subroutine }\end{array}$ \\
\hline$c_{S}$ & $-\cdots$ & 3.5 .9 & $g m / g m$ & Grams of species of interest absorbed on one gran of solid \\
\hline e & EM & 3.2 .4 & Dimensiontess & Effective emmisivity across gas gap of a barrier layer \\
\hline$e_{B}$ & EOUTER & 3.2 .5 & Dimensionless & Emissivity at outer surface of barrier gas gap \\
\hline$e_{3}$ & EINNER & 3.2 .5 & Dimensionless & Emissivity at inner surface of barrier gas gap \\
\hline$f(x)$ & -- & 3.5 .52 & $g m / m / m 1$ & $\begin{array}{l}\text { Concentration profile in the backfill at zero inventory } \\
\text { in the waste package }\end{array}$ \\
\hline f & -- & 3.4 .26 & Dimensionless & $\begin{array}{l}\text { Constant in radial displacement equation in STRESS sub- } \\
\text { routine }\end{array}$ \\
\hline$f(t)$ & -- & 3.5 .27 & $g m / y r$ & $\begin{array}{l}\text { Rate of transport of species of interest out of waste } \\
\text { canister }\end{array}$ \\
\hline g & -- & 3.4 .26 & $i^{2}$ & $\begin{array}{l}\text { Constant in radial displacement equation in STRESS sub- } \\
\text { routine }\end{array}$ \\
\hline$h_{0}$ & 112 & 3.5 .3 & $\mathrm{~cm}^{-1}$ & $\begin{array}{l}\text { Mass conductance at } x=0 \text { divided by diffusion coefficient } \\
\text { for species of interest in the backfill }\end{array}$ \\
\hline$n_{u}$ & IIL & 3.5 .2 & $\mathrm{~cm}^{-1}$ & $\begin{array}{l}\text { Mass conductance at } x=\ell \text { divided by diffusion coefficient } \\
\text { for species of interest in the backfill }\end{array}$ \\
\hline$h_{B R}$ & COCFF & 3.2 .7 & Watts $/$ in $^{2}-{ }^{o} K$ & $\begin{array}{l}\text { Estimated overall heat transfer coefficient between } \\
\text { repository and failed barrier surface }\end{array}$ \\
\hline k & $-\cdots$ & 3.5 .1 & $\mathrm{cmll} / \mathrm{yr}$ & Constant in Equation $(3.5 .1)$ in RELEAS subroutine \\
\hline$k_{c}$ & $-\cdots$ & 3.5 .44 & $\mathrm{~cm} / \mathrm{yr}$ & Constant in Equation (3.5.44) in RELEAS subroutine \\
\hline
\end{tabular}




\begin{tabular}{|c|c|c|c|c|}
\hline SYMBOL & $\begin{array}{l}\text { PROGRAM } \\
\text { VARIABLE }\end{array}$ & EQUATION & UNITS & EXPLANATION \\
\hline$k_{p}$ & --- & --- & $\mathrm{cm} / \mathrm{min}$ & Permeability of porous media \\
\hline$k$ & K & 3.5 .12 & $\mathrm{~cm}^{2} / \mathrm{yr}$ & $\begin{array}{l}\text { Constant in exponential terms which generate transient } \\
\text { response of series in RELEAS subroutine }\end{array}$ \\
\hline$k_{d}$ & KD & 3.5 .9 & $\mathrm{In} 1 / \mathrm{gm}$ & $\begin{array}{l}\text { Equilibrium constant relating concentration of species of } \\
\text { interest in liquid to that absorbed on solid in RELEAS }\end{array}$ \\
\hline$k^{A l}$ & $\operatorname{MCOND(MAT)}$ & 3.2 .3 & Watt/in- ${ }^{0} \mathrm{~K}$ & Thermal conductivity of innermost barrier layer \\
\hline$k^{12}$ & MCOND(MAT2) & 3.2 .3 & Watt/in- ${ }^{0} \mathrm{~K}$ & Thermal conductivity of second innermost barrier layer \\
\hline$k^{23}$ & FCOND(BAK) & 3.2 .3 & Watt/in- ${ }^{0} \mathrm{~K}$ & Thermal conductivity of filler or backfill \\
\hline$k^{3 B}$ & MCOND(MATGAP) & 3.2 .4 & Watt/in- ${ }^{0} K$ & Thermal conductivity of barrier gas gap \\
\hline$\ell$ & $L$ & 3.5 .1 & $\mathrm{~cm}$ & Thickness of Backfill in RELEAS subroutine \\
\hline$q$ & -- & 3.5 .15 & $\mathrm{gin} / \mathrm{yr}-\mathrm{cm}^{2}$ & Transport flux through a slab in RELEAS subroutine \\
\hline$r$ & -- & -- & inches & $\begin{array}{l}\text { Radius of barrier package layer relative to waste } \\
\text { centerline }(r=0)\end{array}$ \\
\hline$r_{A}$ & IDIAM & 3.2 .3 & inches & Radius of inner surface of innermost barrier layer \\
\hline$r_{B}$ & GDIAM & 3.2 .3 & inches & Radius of outer surface of barrier gas gap \\
\hline$r_{1}$ & ODIAM & 3.2 .3 & inches & Radius of outer surface of innermost barrier layer \\
\hline$r_{2}$ & ODIAM2 & 3.2 .3 & inches & Radius of outer surface of second innermost barrier layer \\
\hline$r_{3}$ & FDIAil & 3.2 .3 & inches & Radius of outer surface of filler or backfill \\
\hline$r_{\text {tr }}$ & $-\cdots$ & 3.5 .30 & $g m / y r$ & $\begin{array}{l}\text { Constant rate of transport of species of interest out or } \\
\text { waste canister }\end{array}$ \\
\hline$t$ & TIME & 3.2 .1 & yr & Time elapsed since package emplacement \\
\hline$t_{f}$ & TFINAL & 3.5 .31 & yr & Time when quantity of radionuclides in waste is zero \\
\hline$t_{s}$ & TS & 3.5 .25 & yr & $\begin{array}{l}\text { Time at which constant transport conditions prevail } \\
\text { (steady state time) in RELEAS subroutine }\end{array}$ \\
\hline$t^{\prime}$ & $-\cdots$ & 3.5 .35 & yr & Time elapsed since package emplacement \\
\hline$u(x)$ & EXACT & 3.5 .17 & $g m / m 1$ & $\begin{array}{l}\text { Concentration profile in backfill at constant transport } \\
\text { conditions (steady state profile) }\end{array}$ \\
\hline
\end{tabular}




\begin{tabular}{|c|c|c|c|c|}
\hline SYMBOL & $\begin{array}{l}\text { PROGRAM } \\
\text { VARIABLE }\end{array}$ & EQUATION & UNITS & EXPLANATION \\
\hline$v(x, 0)$ & $-\cdots$ & 3.5 .52 & $\mathrm{gm} / \mathrm{m} 1$ & $\begin{array}{l}\text { Concentration profile in the backfill for the insulated } \\
\text { condition }\end{array}$ \\
\hline$w(x, t)$ & SERIES & 3.5 .17 & $\mathrm{gm} / \mathrm{mll}$ & Time-dependent concentration profile in the backfill \\
\hline$x$ & $x$ & 3.5 .1 & $\mathrm{~cm}$ & Distance from arbitrary reference point \\
\hline$x_{0}$ & THICK, THICK2 & 3.3 .1 & inches & Previous barrier layer thickness \\
\hline$x_{1}$ & THICK, THICK2 & 3.3 .1 & inches & $\begin{array}{l}\text { New barrier layer thickness after corrosion over an } \\
\text { increment of time }\end{array}$ \\
\hline$y_{0}$ & -- & 3.5 .28 & gm & Initial quantity of waste in RELEAS subroutine \\
\hline y & Y & 3.5 .27 & $g m$ & $\begin{array}{l}\text { Quantity of species of interest remaining in waste before } \\
\text { constant transport prevails in RELEAS subroutine }\end{array}$ \\
\hline$z_{s}$ & $-\cdots$ & 3.5 .48 & $\mathrm{~cm}$ & $\begin{array}{l}\text { Location of soluble substance-solution interface in a } \\
\text { solid in RELEAS subroutine }\end{array}$ \\
\hline$z$ & -- & 3.5 .30 & gin & $\begin{array}{l}\text { Quantity of species of interest remaining in waste after } \\
\text { constant transport conditions prevail in RELEAS subroutine }\end{array}$ \\
\hline$z$ & -- & 3.6 .1 & $\mathrm{~cm}$ & Self-shielding distance factor in RADCLC subroutine \\
\hline $\mathrm{z}_{0}$ & Y & 3.5 .31 & gm & $\begin{array}{l}\text { Quantity of species of interest in waste at time when } \\
\text { constant transport conditions prevail in RELEAS subroutine }\end{array}$ \\
\hline$\ddot{z}$ & -- & 3.4 .24 & in $/ \sec ^{2}$ & $d^{2} L / d t^{2}$ \\
\hline$\alpha$ & $-\cdots$ & 3.5 .21 & $\mathrm{~cm}^{-1}$ & Parameter in Fourier series in RELEAS subroutine \\
\hline$x_{1}$ & -- & 3.5 .25 & $\mathrm{~cm}^{-1}$ & Parameter in Fourier series in RELEAS subroutine \\
\hline$x_{n}$ & $R(N)$ & 3.5 .19 & $\mathrm{~cm}^{-1}$ & $\begin{array}{l}\text { Parameter in } n \text {-th term of Fourier series in RELEAS sub- } \\
\text { routine }\end{array}$ \\
\hline$\beta_{j}$ & -- & 3.5 .53 & $\mathrm{~cm}^{-1}$ & $\mathrm{~N}$-th positive root of Equation $(3.5 .54)$ \\
\hline$\beta$ & BETA & 3.4 .1 & Dimension less & Constant slope of Mohr envelope in yield model of STRESS \\
\hline$\nu$ & POISS & 3.4 .8 & Dimension less & Poisson's ratio in STRESS subroutine \\
\hline$\Delta r$ & THICK & 3.2 .7 & inches & Thickness of inner barrier layer (wall) at time of failure \\
\hline
\end{tabular}




\begin{tabular}{|c|c|c|c|c|}
\hline SYMBOL & $\begin{array}{l}\text { PROGRAM } \\
\text { VARIABLE }\end{array}$ & EQUATION & UNITS & EXPLANATION \\
\hline$\Delta \mathrm{t}$ & DELTA & 3.31 & yr & Time increment \\
\hline$\Lambda_{O} R$ & -- & 3.4 .16 & Ksi & Change in radial stress - STRESS subroutine \\
\hline$\Delta \sigma_{Z}$ & -- & 3.4 .18 & Ksi & Change in axial stress - STRESS subroutine \\
\hline$\Lambda s_{0}$ & $-\infty$ & 3.4 .17 & Ksi & Change in angular stress - STRESS subroutine \\
\hline$b$ & TORTUR & 3.5 .6 & Dimensionless & $\begin{array}{l}\text { Tortuosity of porous medium with respect to diffusion in } \\
\text { RELEAS subroutine }\end{array}$ \\
\hline$\delta$ & THICK & 3.4 .44 & inches & $\begin{array}{l}\text { Wall thickness used in hoop stress calculation in STRESS } \\
\text { subroutine }\end{array}$ \\
\hline${ }^{z} z$ & $-\cdots$ & 3.4 .36 & in $/$ in & Change in axial strain at $R_{2}$ in STRESS subroutine \\
\hline$\varepsilon$ & EP & 3.4 .16 & Dimensionless & Void volume of porous inedium in RELEAS subroutine \\
\hline${ }^{\varepsilon} \mathrm{R}$ & -- & 3.5 .16 & in/in & Change in radial strain - STRESS subroutine \\
\hline$\iota_{z}$ & $-\cdots$ & 3.4 .16 & $\mathrm{in} / \mathrm{in}$ & Change in axial strain - STRESS subroutine \\
\hline$\because$ & -- & 3.4 .16 & in/in & Change in angular strain - STRESS subroutine \\
\hline 1 & ETA & 3.4 .38 & Dimensiontess & Constant defined by Equation (3.4.39) in STRESS subroutine \\
\hline$\lambda_{n}$ & $-\cdots$ & 3.5 .40 & Dimensionless & Parameter in RELEAS subroutine, $\lambda_{n}=n l l$ \\
\hline$\lambda_{m}$ & --- & 3.5 .65 & Dimensionless & Parameter in RELEAS subroutine, $\lambda_{m}=m I l$ \\
\hline 1 & LAMBDA & 3.4 .16 & Ksi & Lamé constant for barrier wall in STRESS subroutine \\
\hline$\lambda$ & DECAYC & 3.5 .27 & $y r^{-1}$ & $\begin{array}{l}\text { Radiodecay constant of species of interest in RELEAS } \\
\text { subroutine }\end{array}$ \\
\hline$\lambda$ & BLMB & 3.4 .10 & Ksi & Lame constant for backfill in STRESS subroutine \\
\hline p & CLAYD & 3.5 .11 & $\mathrm{gm} / \mathrm{ml}]$ & Bulk density of porous medium in RELEAS subroutine \\
\hline 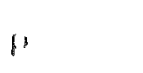 & $-\cdots$ & 3.4 .23 & $\mathrm{lb} / \mathrm{in}^{3}$ & Density \\
\hline () & $-\cdots$ & 3.2 .4 & watt/in ${ }^{2}-{ }^{0} K^{4}$ & Boltzinan constant \\
\hline a & $-\cdots$ & 3.5 .66 & Diménsionless & Parameter in RELEAS subroutine, $0=k \mathrm{t} / \ell^{2}$ \\
\hline$(x)$ & -.. & 3.5 .44 & $\mathrm{~cm}$ & $\begin{array}{l}\text { Infinite transport distance in a slab in RELEAS } \\
\text { subroutine }\end{array}$ \\
\hline
\end{tabular}




\begin{tabular}{|c|c|c|c|c|}
\hline SYMBOL & $\begin{array}{l}\text { PROGRAM } \\
\text { VARIABLE }\end{array}$ & EQUATION & UNITS & EXPLANATION \\
\hline$a$ & $-\cdots$ & 3.4 .23 & Inches & Outside radius of cylinder in STRESS subroutine \\
\hline "R & --- & 3.4 .16 & Ksi & Radial stress in STRESS subroutine \\
\hline $1_{z}$ & $-\cdots$ & 3.4 .18 & Ksi & Axial stress in STRESS subroutine \\
\hline 10 & -- & 3.4 .17 & Ksi & Angular stress in STRESS subroutine \\
\hline$o_{R}^{O}$ & $-\cdots$ & 3.4 .19 & Ksi & Initial radial stress in STRESS subroutine \\
\hline a事 & -- & 3.4 .20 & Ksi & Initial axial stress in STRESS subroutine \\
\hline 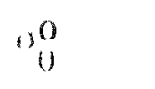 & $-\cdots$ & 3.4 .19 & Ksi & Initial angular stress in STRESS subroutine \\
\hline BAK & BAK & $-\cdots$ & Dimensionless & Identification code for backfill material type \\
\hline BTAIL & BFAIL & $-\cdots$ & Dimensionless & $\begin{array}{l}\text { Designates backfill integrity in STRESS subroutine } \\
(=0 \text { indicates failure })\end{array}$ \\
\hline CLPRES & CLPRES & $-\cdots$ & ps $\mathbf{i}$ & Internal pressure on barrier at time of repository sealing \\
\hline CLTEMP & CLTEMP & -- & ${ }^{o} \mathrm{~K}$ & $\begin{array}{l}\text { Internal temperature on barrier at time of repository } \\
\text { sealing }\end{array}$ \\
\hline COAT & COAT & $-\cdots$ & $y r$ & Coating delay time in CORODE subroutine \\
\hline rLUX & rLUX & 3.6 .1 & photons $/ \mathrm{cm}^{2} / \mathrm{sec}$ & Gamma ray flux at a particular package location \\
\hline IB & IB & $-\cdots$ & Dimensionless & $\begin{array}{l}\text { Number of barriers in a package design case }(I=I B \text { for } \\
\text { outernost barrier) }\end{array}$ \\
\hline IGE & $\mathrm{IGL}$ & $-\cdots$ & Dimensiontess & Identification code for geology \\
\hline IL & II. & 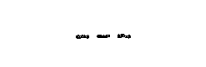 & Dimensiontess & Identification code for type of barrier \\
\hline IWATER & IWATER & -- & Dimensionless & $\begin{array}{l}\text { Identification code for type of repository } \\
\text { water }\end{array}$ \\
\hline MAT & MAT & $-\cdots$ & Dimensiontess & $\begin{array}{l}\text { Identification code for type of material in inner barricr } \\
\text { layer }\end{array}$ \\
\hline MAT2 & MAT2 & $-\cdots$ & Dimensionless & $\begin{array}{l}\text { Identification code for type of material in second inner- } \\
\text { most barrier layer }\end{array}$ \\
\hline
\end{tabular}




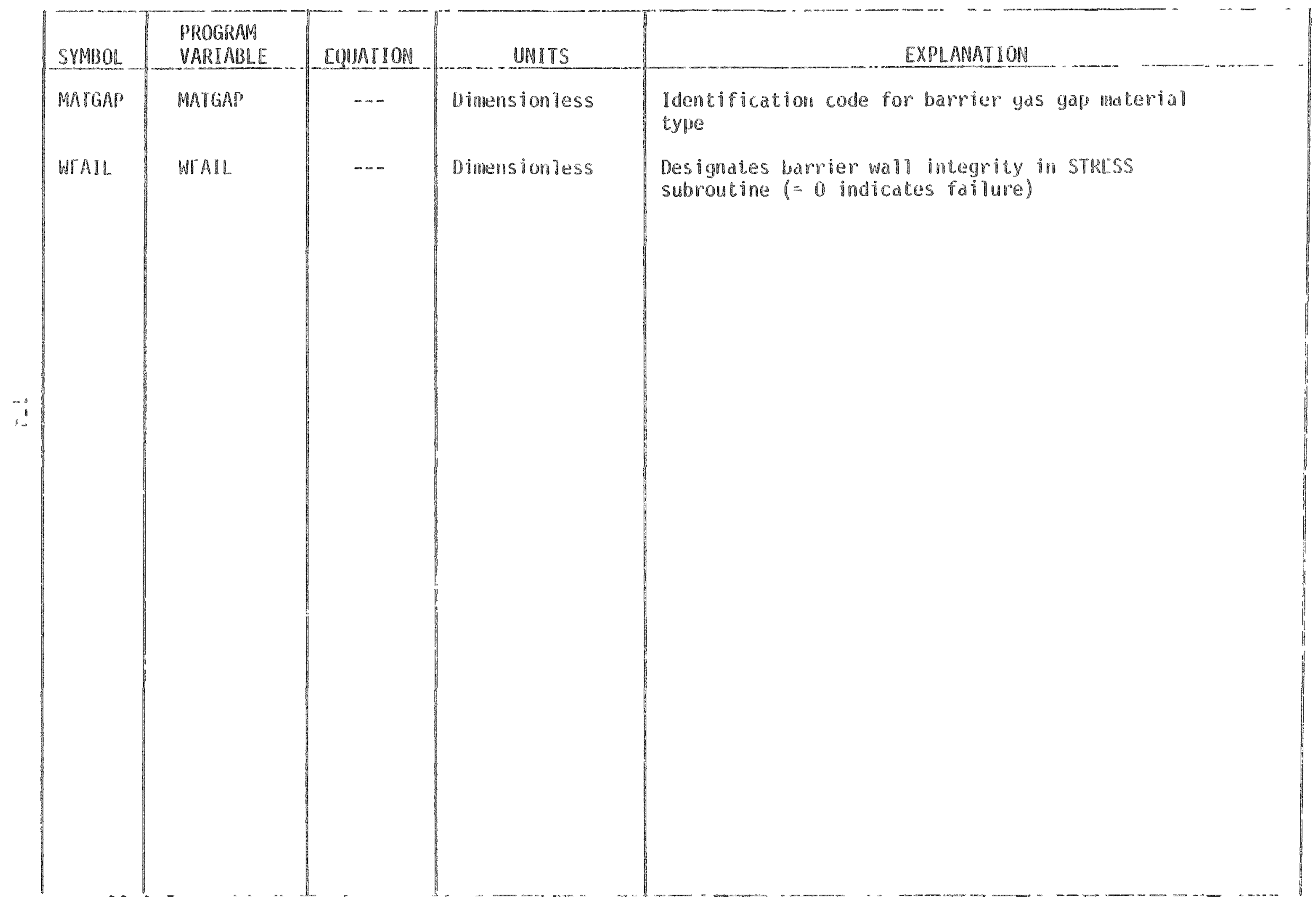


APPENDIK A

BARIER PROGRAM LISTING

The program listing of BARIER and all of its subroutines is provided in this Appendix. 


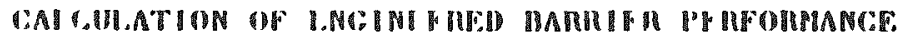

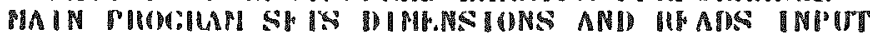

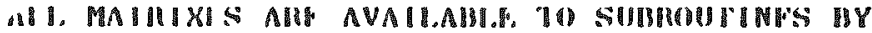

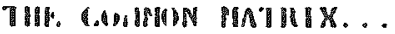

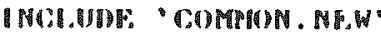

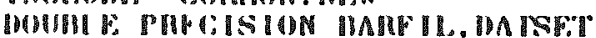

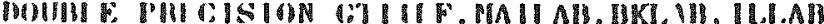

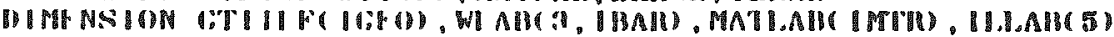
DKI.AII IMBAK 


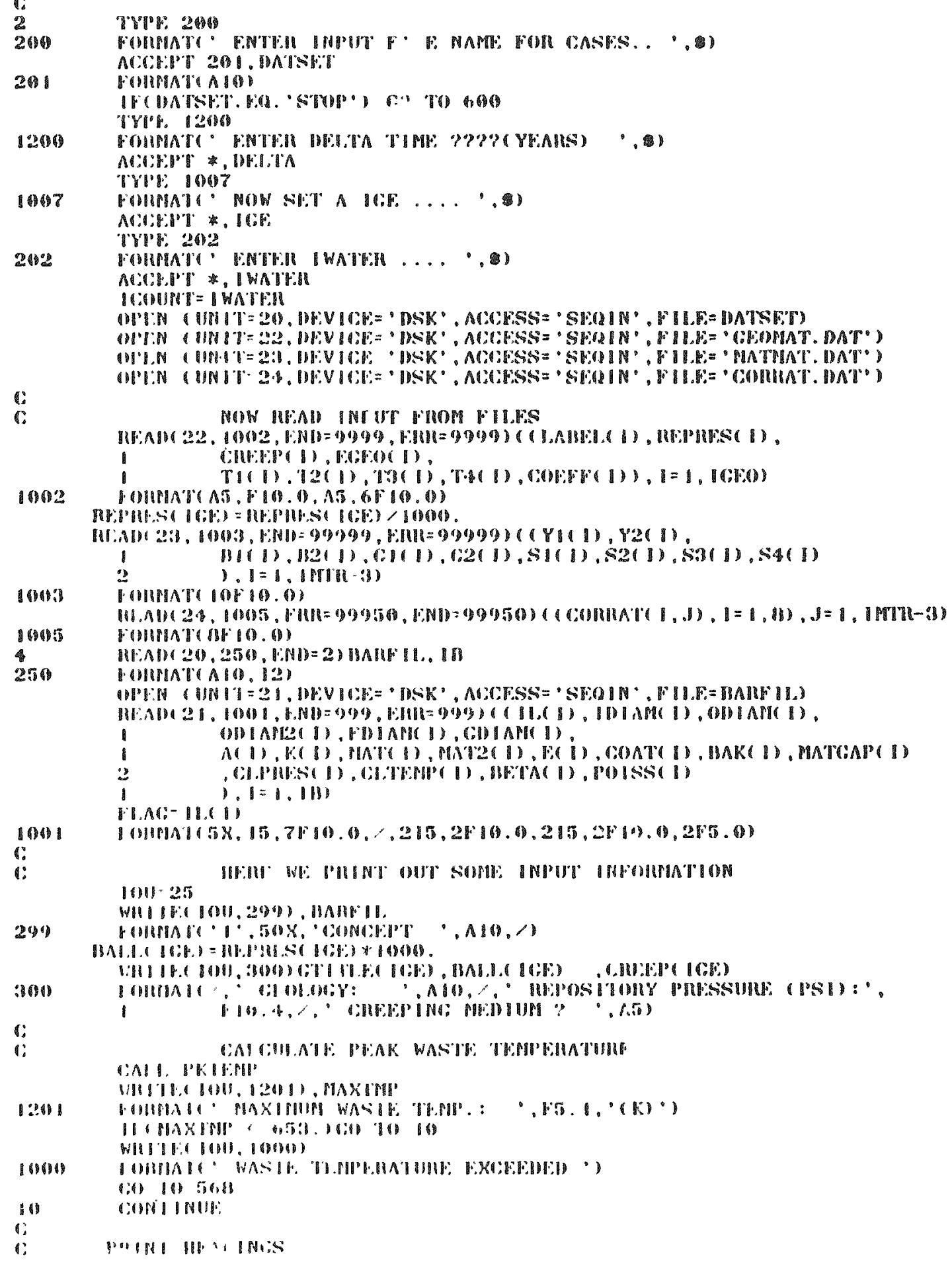




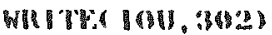

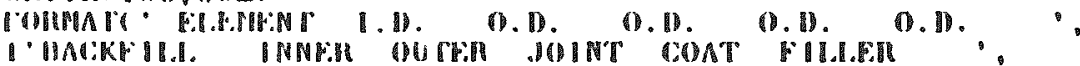

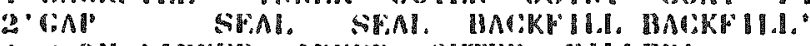

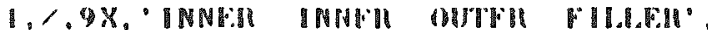

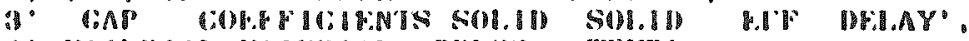

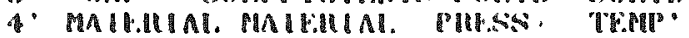

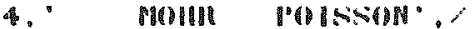

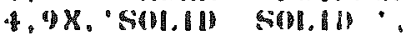

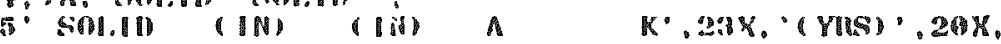

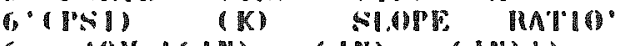

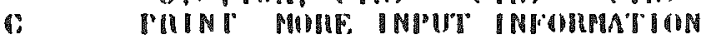

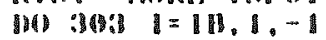

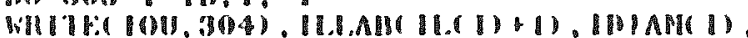

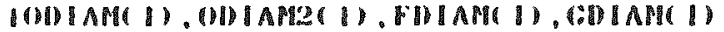

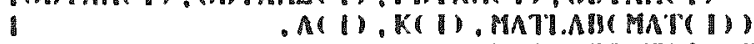

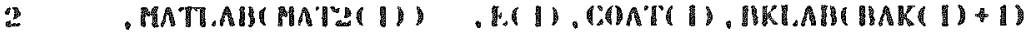

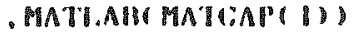

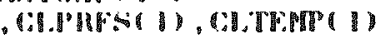

96)

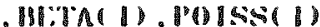

CMNTINIT:

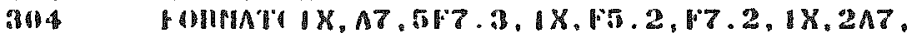
1 1. 5.

(10.2.

WIV

6

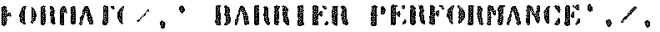
- 2

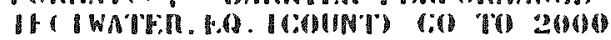

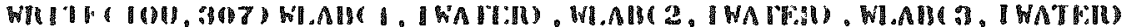

9197

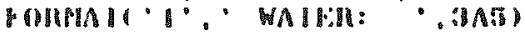

(5) 101080

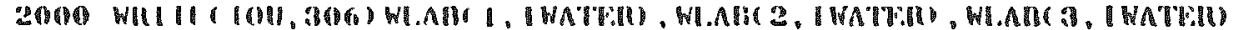

Iิ6

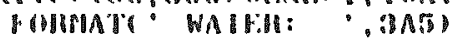

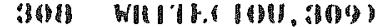

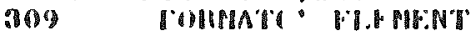

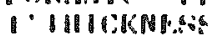

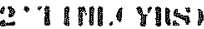

B. ITHER

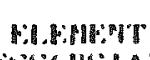

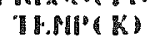

WAIIIIE

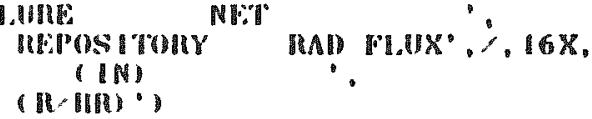

?

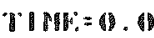

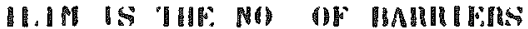

IIBCHE-

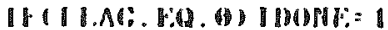

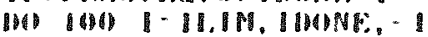

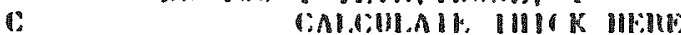

UIIT:E-

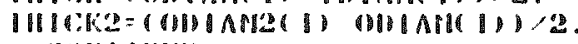

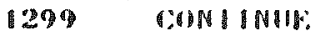

$\| \mathrm{AH} \cdot-1$

WHW $=1$

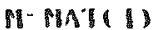

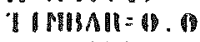

7

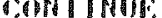

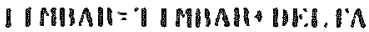

IIMI. IBII. HIVI. IA

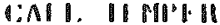

G:AII. MAMU:I:

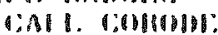

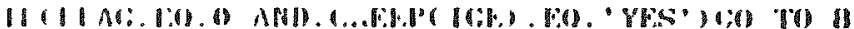

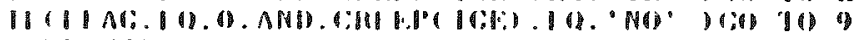

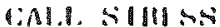

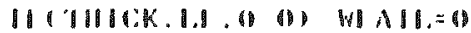

1

(a) 111000

UIII.=

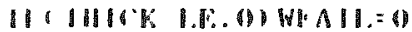

กी III: 


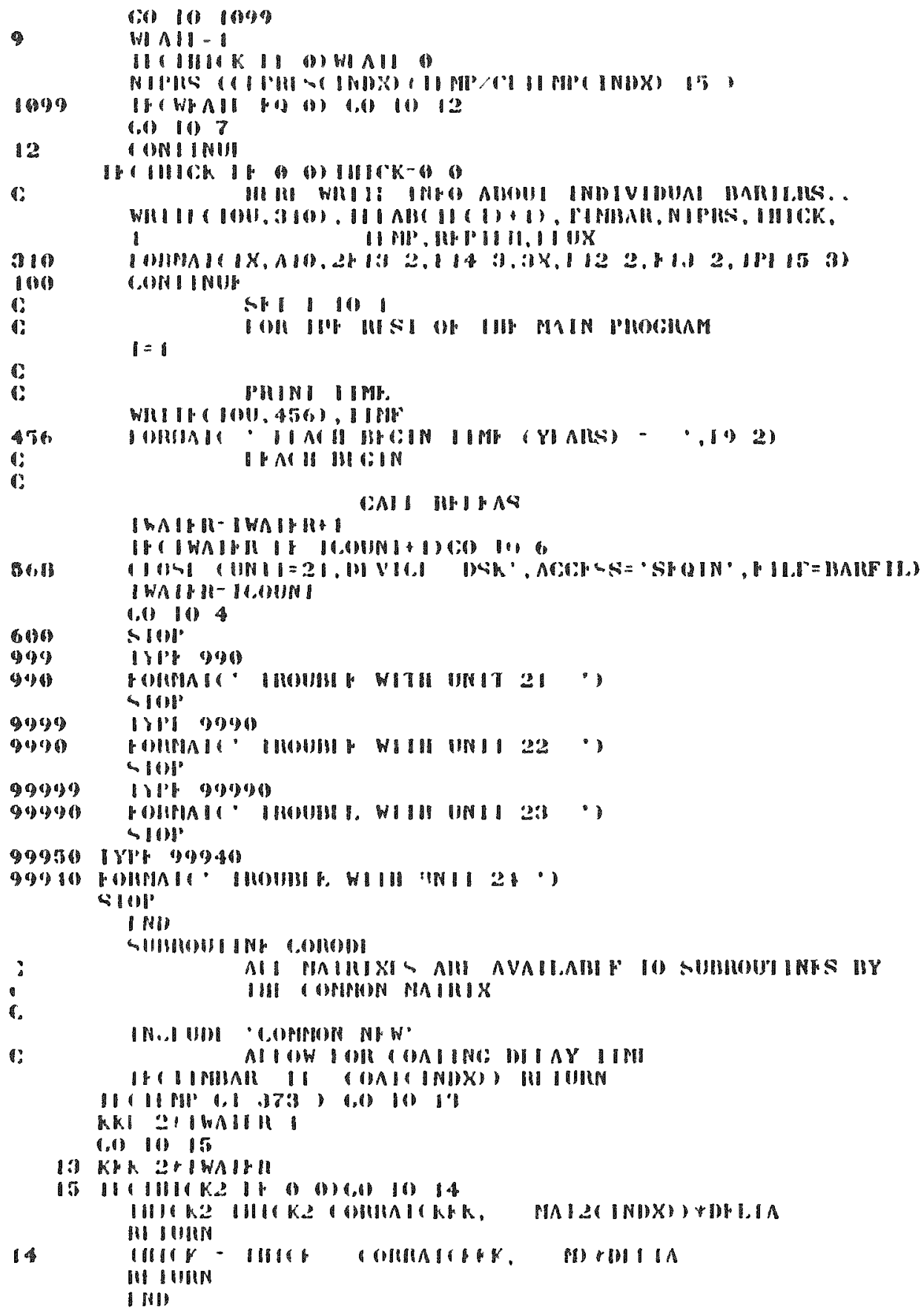




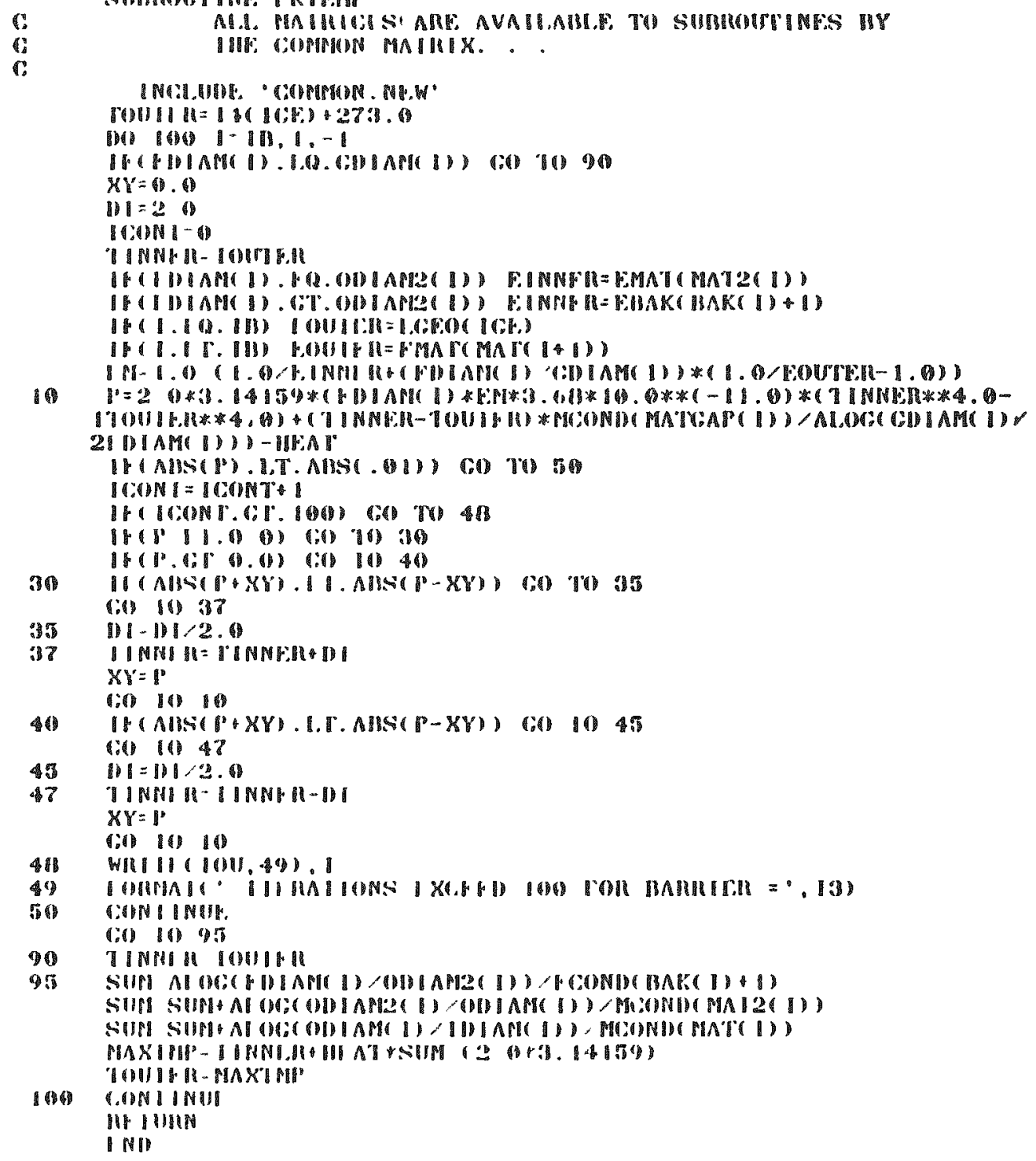




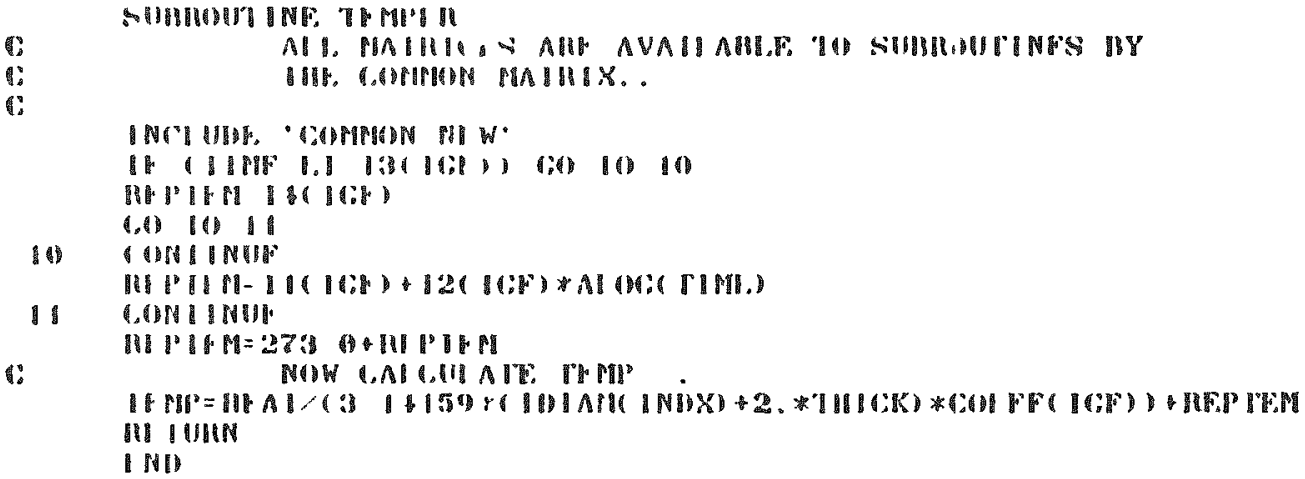


SURROUT INT, STRFSS

INA.UDF, COPPYN. NEW"

RO $=11$ I AMC B BNX, 2 .

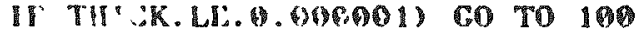

$R I=R O+T I I C K K$

$M 2=\|\| M M E,(I N D X) / 2$.

$R 3=F D \backslash A M(\mathbb{N D X}) / 2$.

TCFNT $=T H M P-273$.

IF(CNETE' IGE) . EQ. "NO') GO TO 200

$Y I F, L D=Y 1(M)+Y 2(M) * T C E N T$

BULK $=B 1(\mathrm{M})+B 2(M) *$ TCENT

SIIFAR = F ( M) + G2 (M) * TCENT

IF ( IDIANC ININX)-ODIAME INDX) .LT. .O1) GO TO 140

IF(BFAL.FA.0.0) GO TO BO

IF $\mathrm{K}(\mathrm{NDX}) \cdot \mathrm{LT} \cdot \mathrm{O} .01) \mathrm{GO}$ TO 50

VSTAR $=-\Lambda(I N D X)+$ SOPT $(A(I N D X) * 2,+4 . *$ * $($ INDX) *REPRES (ICE))

VETAR $=$ VSTAR,2. $/ K(I N D X)$

601060

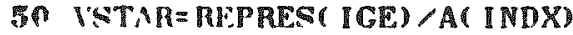

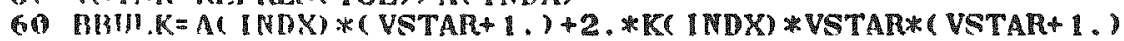

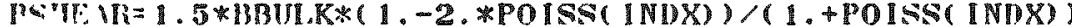

LAMHDA $=$ BUI.K-2. $*$ SIIEAR 3 .

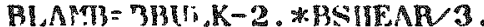

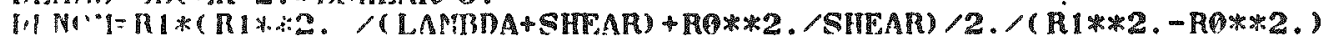

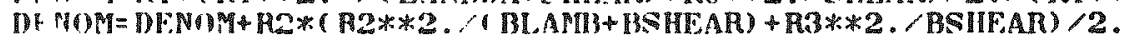
$(\mathrm{Ri} ; * * 2,-\mathrm{R} ; * * 2)$

DFNOM= DFNOAY . * ( R3**2. - M2**2.)

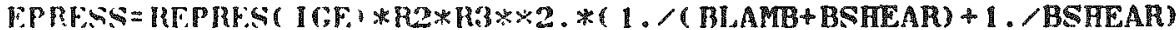

FPRI:AS= TPRPQS. DFENOM

F.TA = SOIT 0.14D148\% BFTA INDX)**2.*(1.-POISS INDX) $* * 2$.

$1-(1 .-2 . * \operatorname{POISS}(\operatorname{INDX}) * 2 . / 3$.

PMINB= REPRFG ( ICF $) *(1 .-$ FTA $) /(1 .-$ ETA*R2**2./R3**2.)

AF( HPITSS. LT. PMINB) GO TO 150

$B H: L=1$

Bn $N U=(3 . * B U L K-2 . * S H T A R) /(3 . * B U L K+S H F A R) / 2$

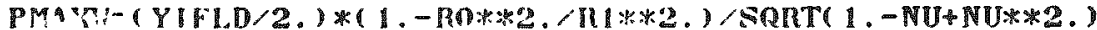

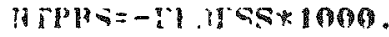

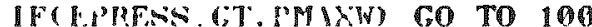

90 WFAIL $=1$

IT. TITN

100 WTAII $=$

RFTIIITN

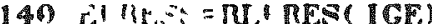

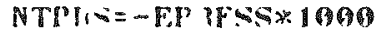

(;) $T: B=$

$1503111=0$

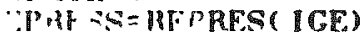

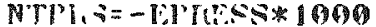

$\mathrm{C}$
$\mathrm{C}$

(i) $70 \mathrm{mo}$

INTERNAI, PRESGURF, DOMINATRS - NO CREFP

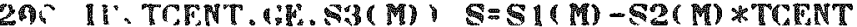

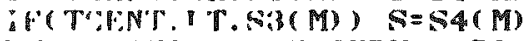

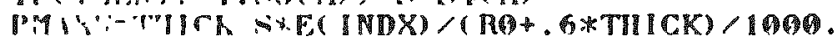

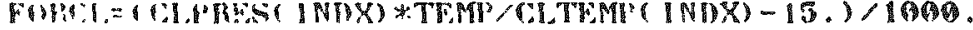

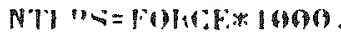

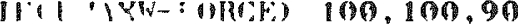

l. III 


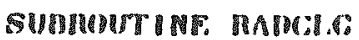

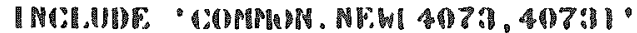

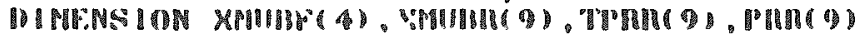

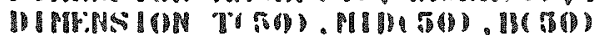

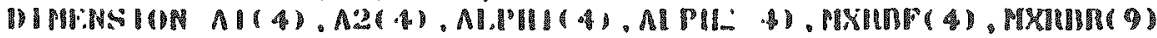

(8)

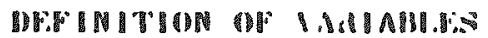

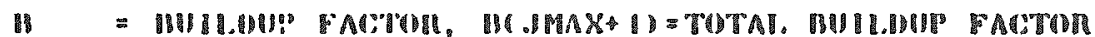

SV = SUUMP:P INIPNSITY

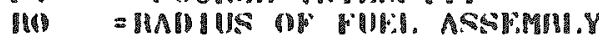

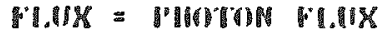

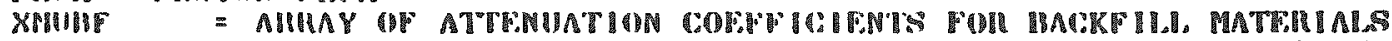

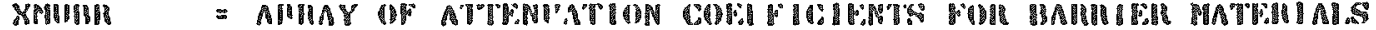

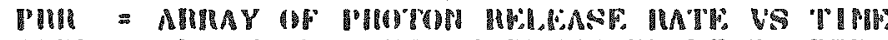

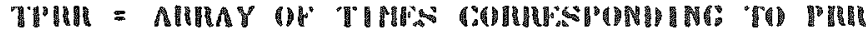

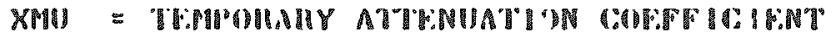

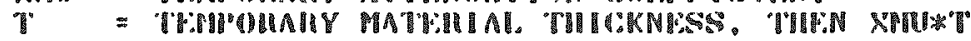

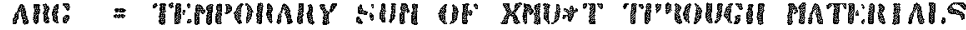

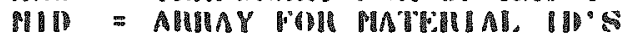

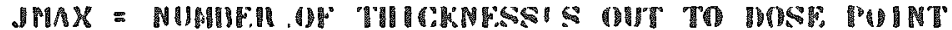

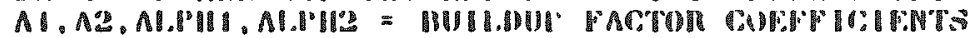

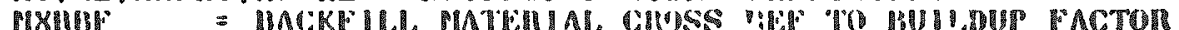

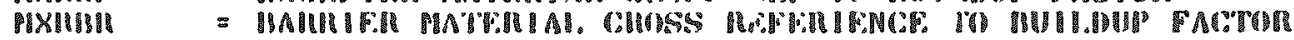

STHP GOMPS

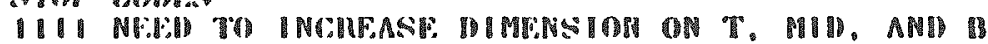

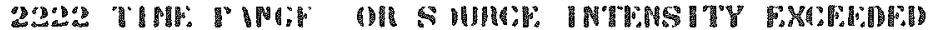

(:)

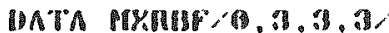

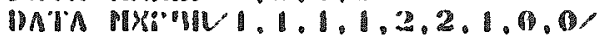

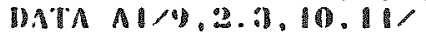

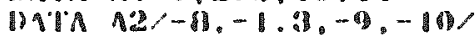

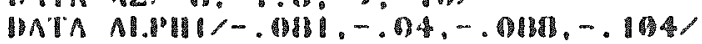

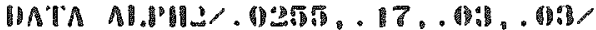

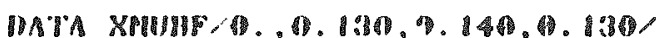

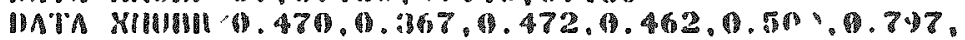

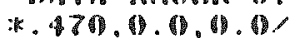

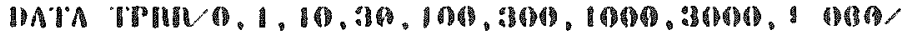

WATA PUIM *2.

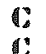

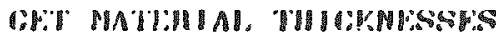

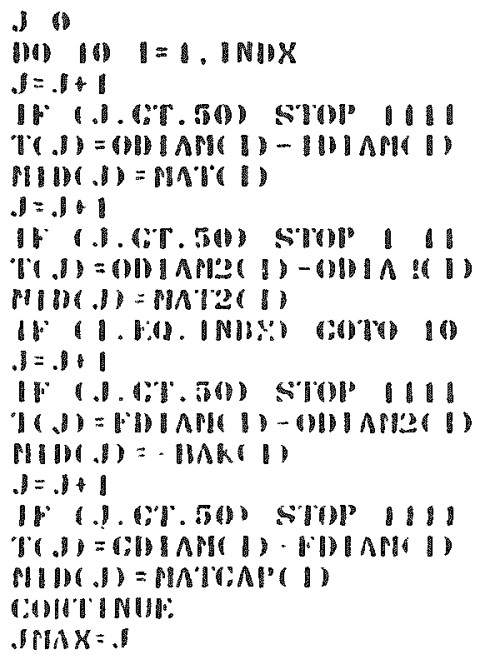




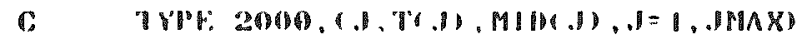

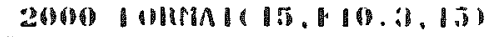

a

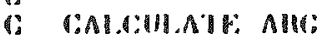

T.

A116:=

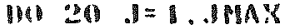

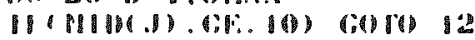

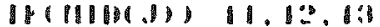

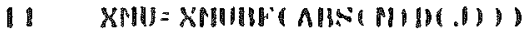

(:)

$12 \quad$ KAHI $=6$.

6001015

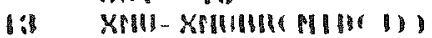

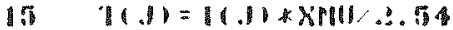

20) Ail:- Mm: TI II)

C: I YUL:

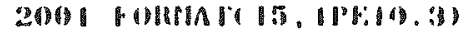

I:

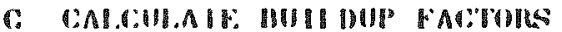

(s)

III) $31=0.50$

$21 \quad$ UI $\|=1$.

$.1=. M A X+B$

MU TH $=1$. IPAX

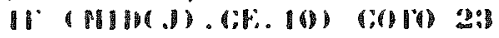

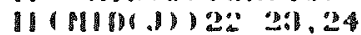

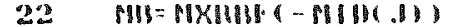

4iAlO

$29 \mathrm{MHO}$

1:1 I I 35

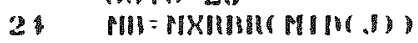

28.

$\| B(I)=1$.

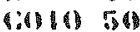

:

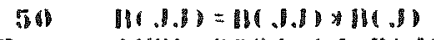

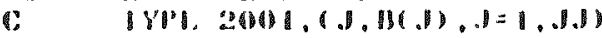

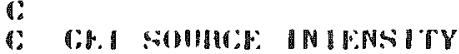

13

III) $1=1.9$

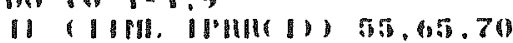

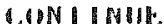

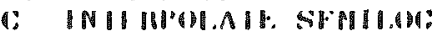

.

$\operatorname{SHBN}=$ IIIISU IMII

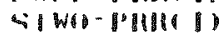

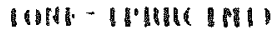

IIT)

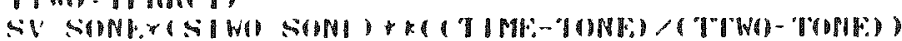

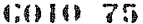

$6058-511811$

1:010 75

$\therefore \|$ |

:

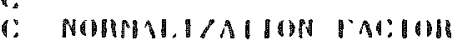

$75 \quad \therefore 8$ il 50110.0919V

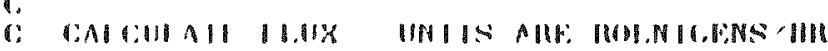

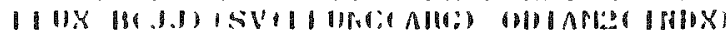

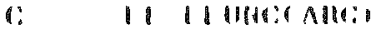

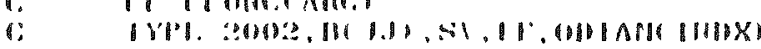

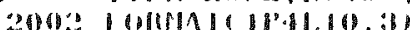

HII $1111 \mathrm{H}$ 
VI:NII

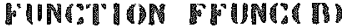

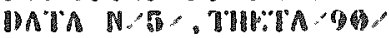

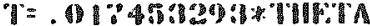

$P P^{*}=$

$B T=M$

IVI II $I=\mathbb{1}, \mathbb{N}$

$X I=(1-1) *\|\|^{2}$

$X 2=\square * T$

$F I=F(X), D)$

$P=R\left(x^{2}, 1\right)$

10

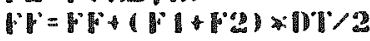

COBNTTHOH,

BVINC:=P HO

IIF:IIIIN

I:PII

FUNG:ION F(X.

$\theta=\cos (x)$

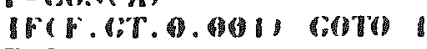

$\mathrm{F}=0$

IIFT TIIIM

$1 \quad \mathbb{P}=-\| \mathrm{B}$

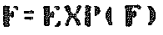

IIr:IIIIIN

PNII 


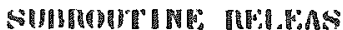

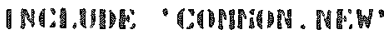

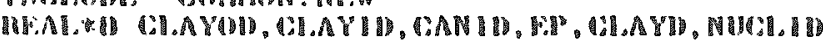

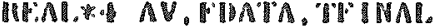

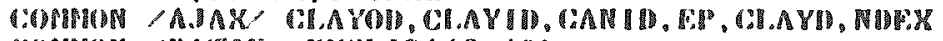

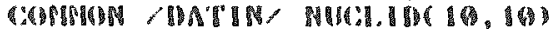

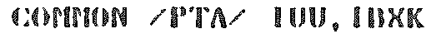

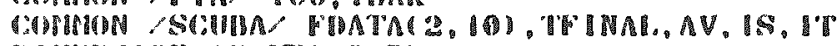

MPHSW

WNTA COH. MU:

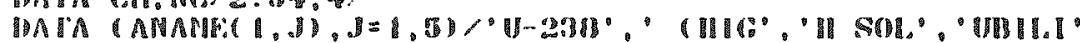

ilvilo

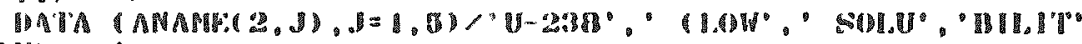

$\because$ P

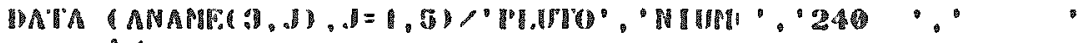

1.

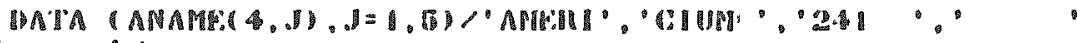

1.

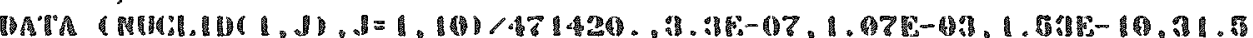

(5)

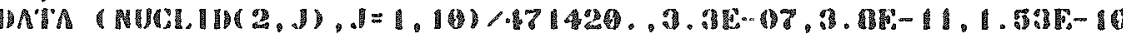

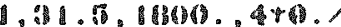

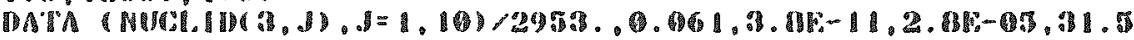

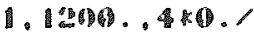

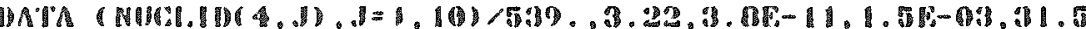

1.40600 .040 .8

$1111=1100$

IIKKK = :

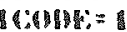

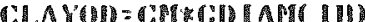

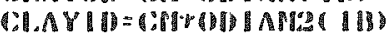

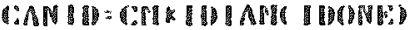

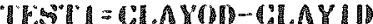

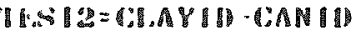

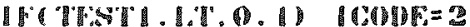

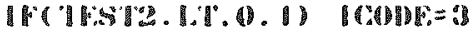

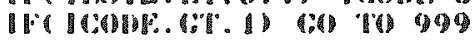

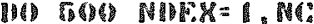

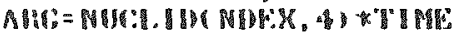

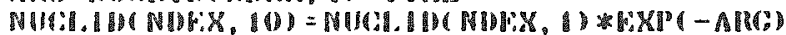

19

SOANT INUTR:

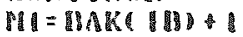

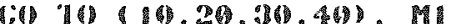

12

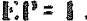

(BH.A Y $=1$.

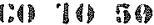

28

$P B=(D)$

$\because A, A Y B=\square$.

(i)

3

$B H^{2}=0$

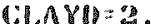

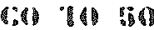

Q:B? $: 1$

COI.AYII: $\mathrm{S}$.

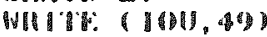

8

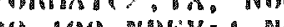

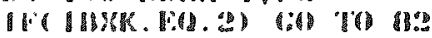

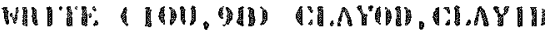

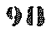

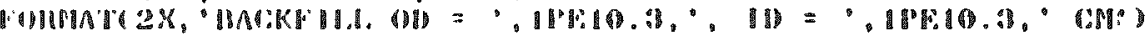

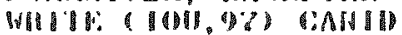

FHITMTY

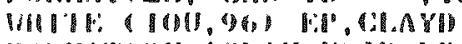

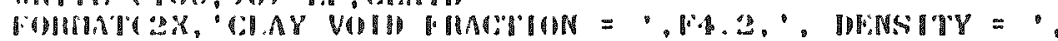

lo.

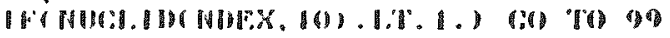




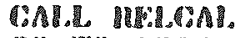

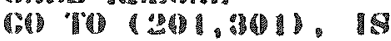

20

$V=\Lambda V \cdot{ }^{2}$

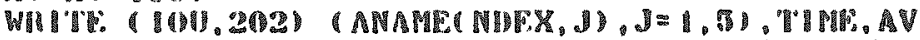

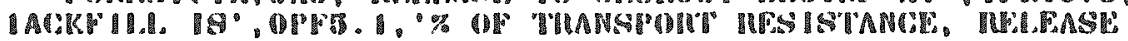

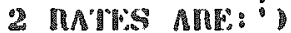

IDO $501 K A=1,10$

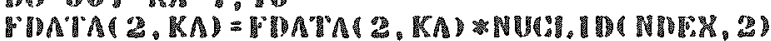

16

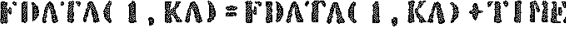

2งเา

2 2.

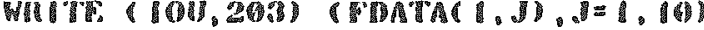

25)

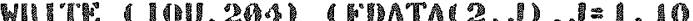

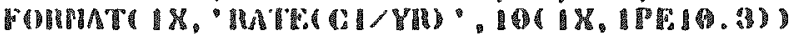

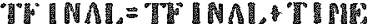

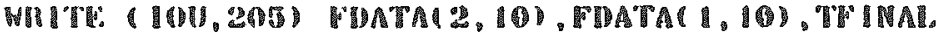

2

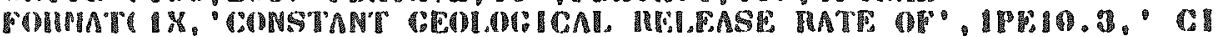

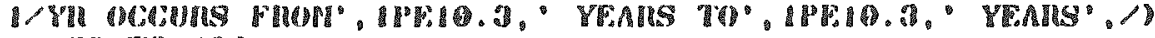

19)

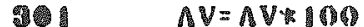

줄눈

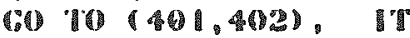

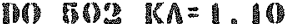

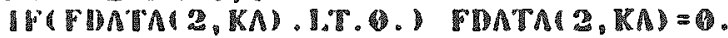

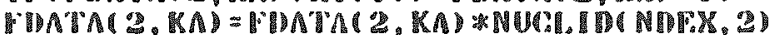

\$62

WHATM

IDI TOS KA=

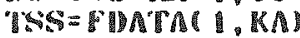

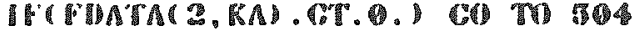

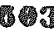
GONTINUTH:

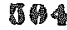

20

ร2) 2

줄

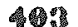

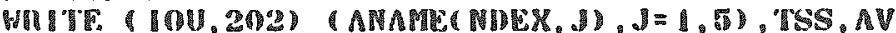

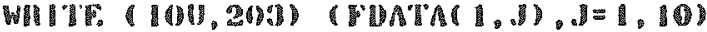

WIIIT: (MII,

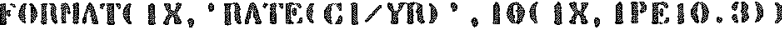

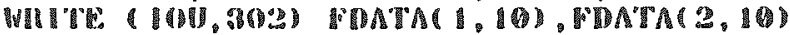

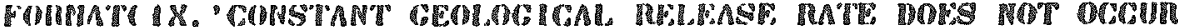

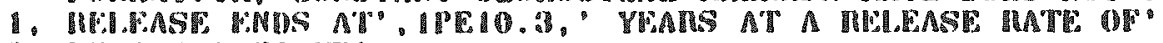

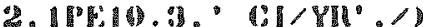

(6) T10

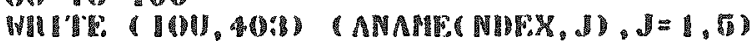

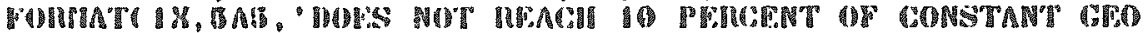

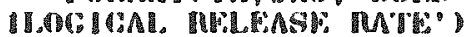

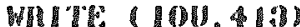

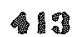

98

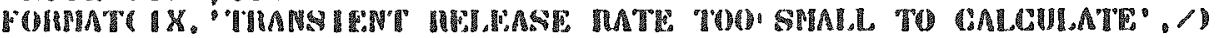
(1)

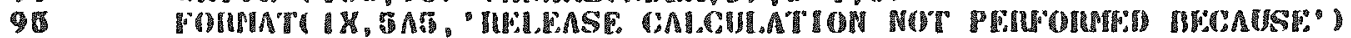

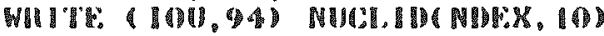

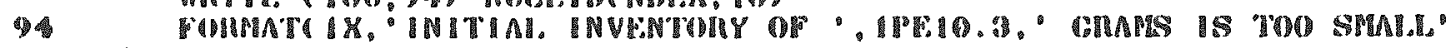
1.1

1 (2)

999 GIV?

HNIII 


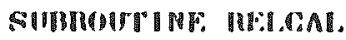

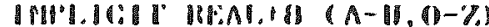

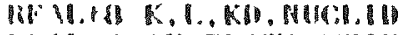

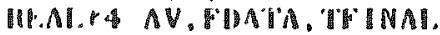

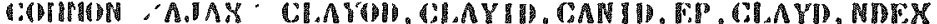

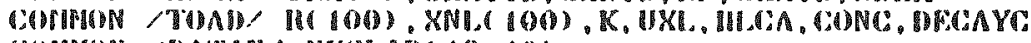

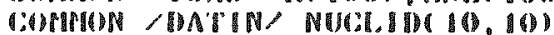

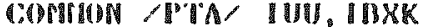

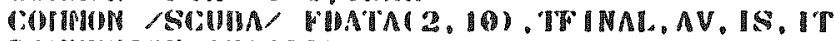

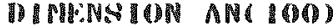

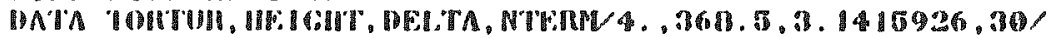

$\| P=1$

$167=110$

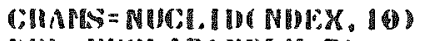

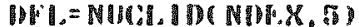

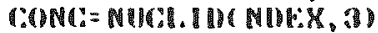

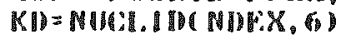

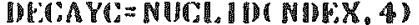

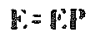

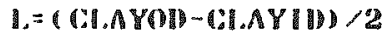

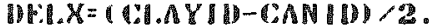

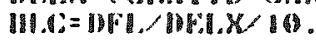

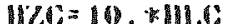

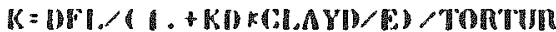

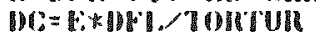

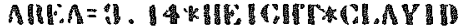

MII:A = MUS:KAME:A

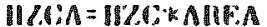

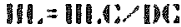

$11 \%=10 .+111$.

$11 \%=112 \% 11 \%$

III. $\mathrm{a}=111 . * 111$.

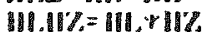

$\Lambda=1 . k 1 . * 411.118$

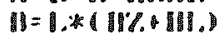

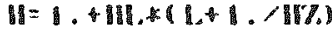

$\mathrm{AV}=\mathbf{I}, *\|11,-1\|$

IT AV.LIT. B GO TO 59

CONM:= GONG:HAV

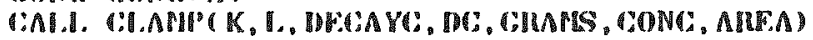

(20) Tी

IE

WHIIL $(1011,6000)$ UII,

610

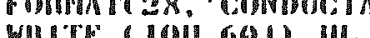

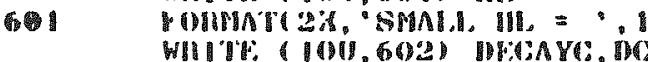

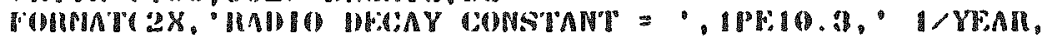

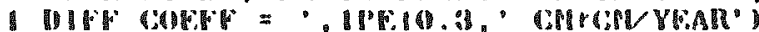

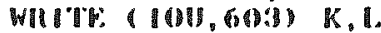

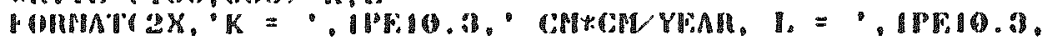

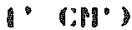

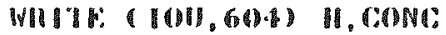

64

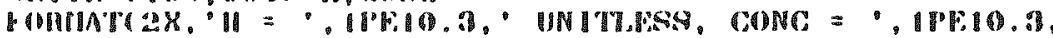

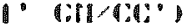

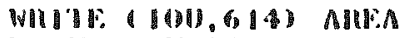

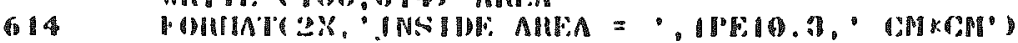

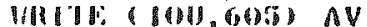

605

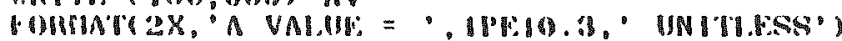

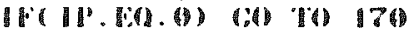

WII II I

169

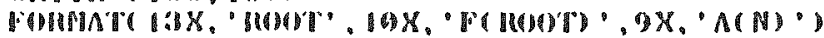

17

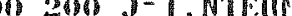

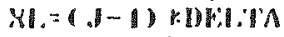

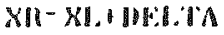

$y=1$. 


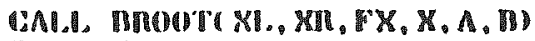

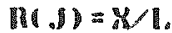

AI.PMA $=$ II II

$M I=$ M.IBIA*I.

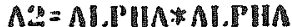

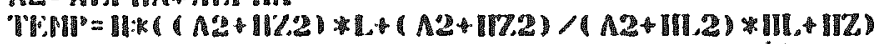

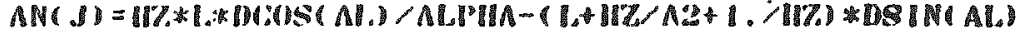

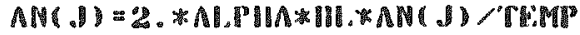

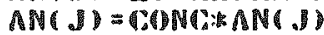

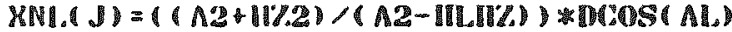

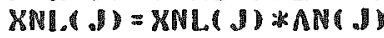

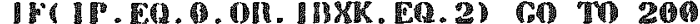

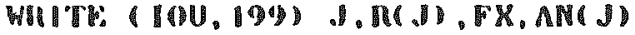

FUMUT

CONTINUE:

TS:

IFI IIXK. ER.21 COTO 202

WIITR ( 1011,201$)$ TS

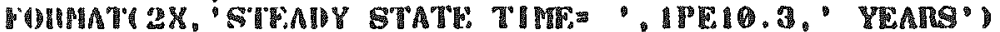

WDIIT: IUU, IT

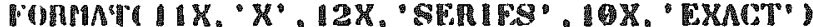

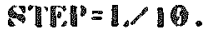

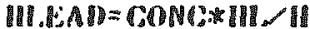

$X=$.

120 $4=1,1$

A

MO $\$ 501$. NTWM

AIPIIA=III $J 1$

$\Lambda X=A M P D A-X$

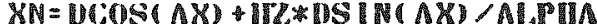

ร9)

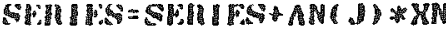

CONN I NUE:

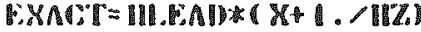

HOIIXK. ION.

WIITE 101, BTI

3

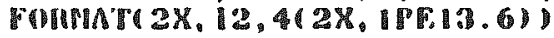

CONN INUT

IIIIXK. EQ.2) CO TO 203

WIITH. (10U, 7. I) CRMPS

22

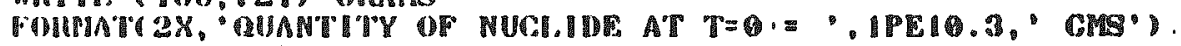

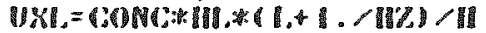

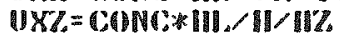

$15=1$

$X I=0$

XII $=115$

$\because x=1$.

(11) $100 \quad K K=1.298$

$X T=(X D+X I / 2$.

GPAS = GOMAPS:

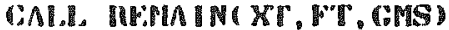

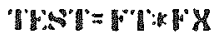

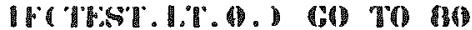

$X I=X 1$

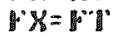

(i) TO 100

190

$\mathrm{XII}=\mathrm{XI}$

19

$B=2$

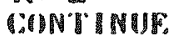

$\mathrm{P} P=1$

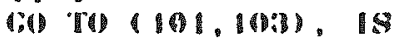

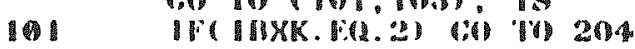

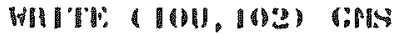

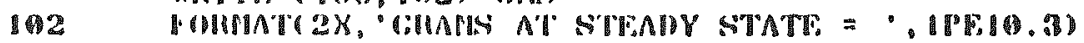

ats

Q⿻日土龰 10 


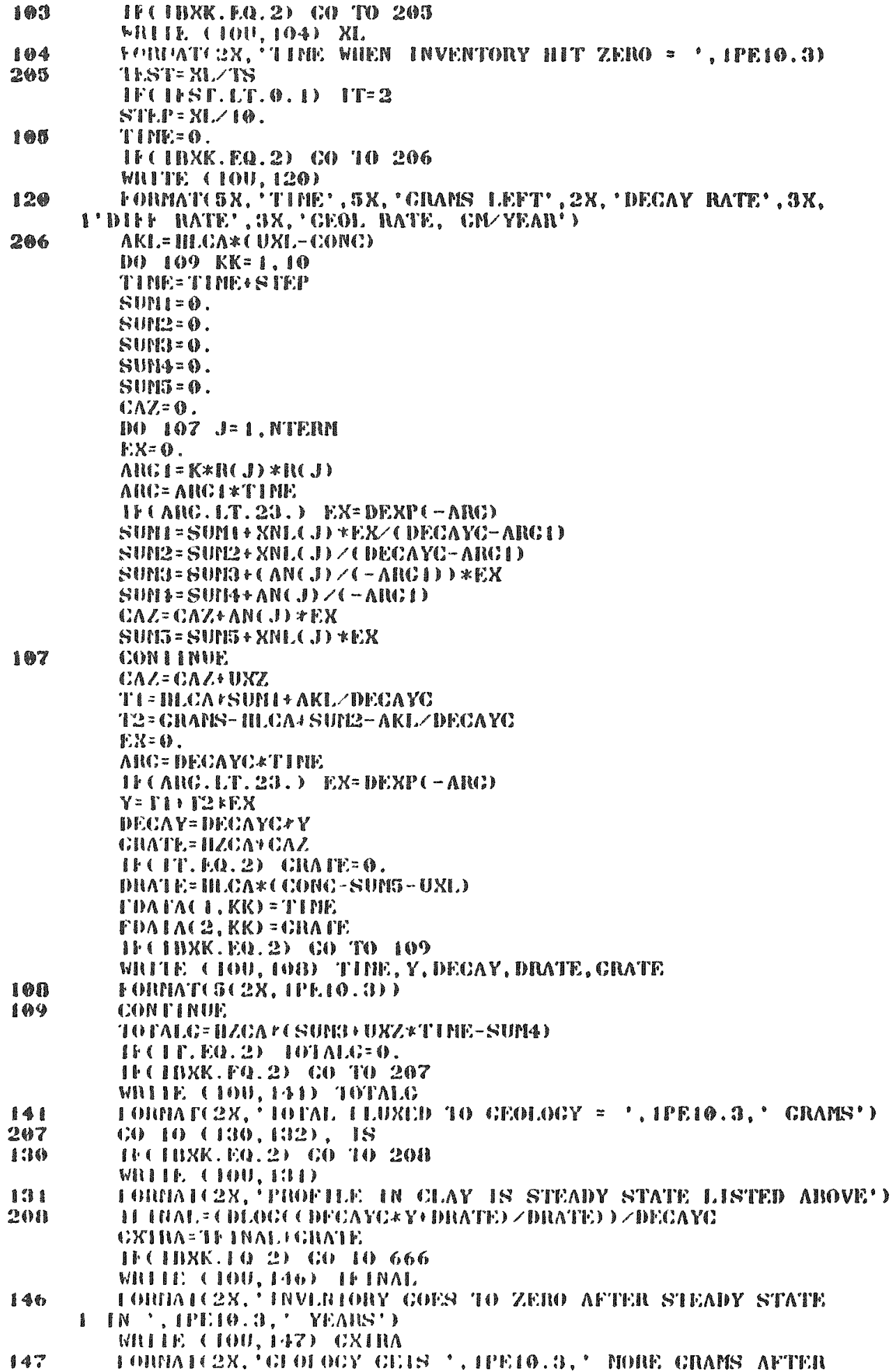

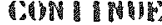

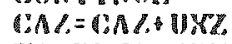

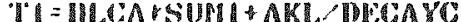

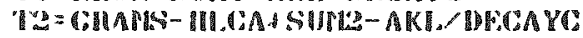

$7: B=$.

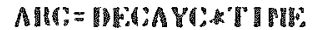

II CMU:-1.T.

$V=I 11 \% k F$

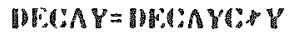

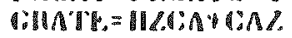

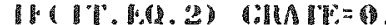

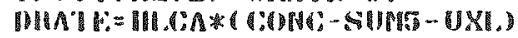

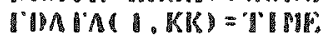

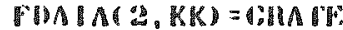

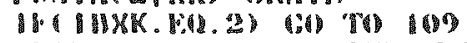

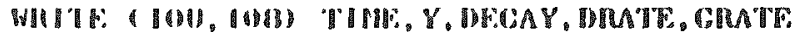

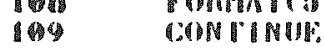

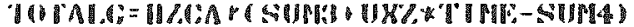

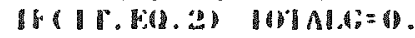

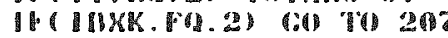

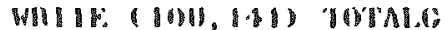

IOUPM

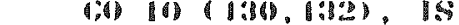

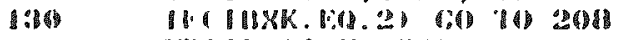

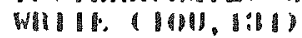

1:

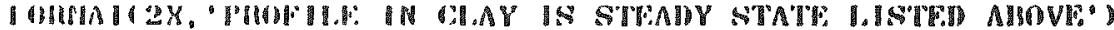

2011

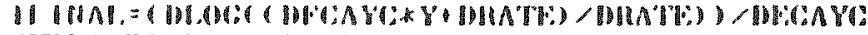

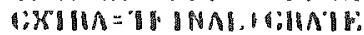

II

14

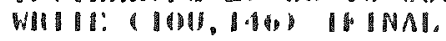

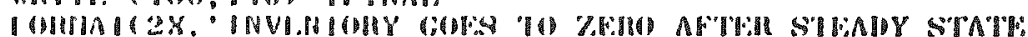

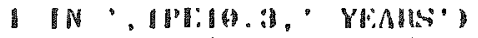

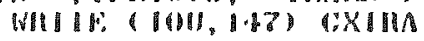


1 STPADY STAT:

4: Th 6ro

$17 \%$

IF IIXK. PO.2) GO TU 200

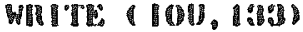

4

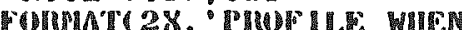

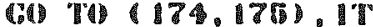

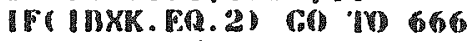

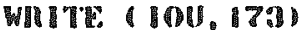

19

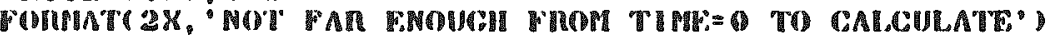

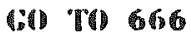

17 둘

19

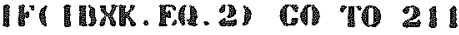

WIIIT?. ( IOH, 134)

21

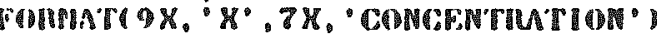

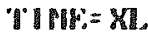

$X=0$.

STP.P $=\mathbb{1}, / 10$.

(1) $139=1,1$

S?:II I IS:

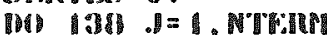

AI.PBIA $=$ III II)

$\Lambda X=M . P D M X$

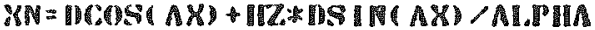

$\mathbb{R}: X=$

AII: = IC

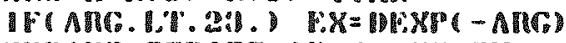

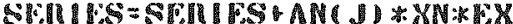

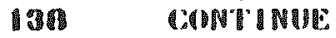

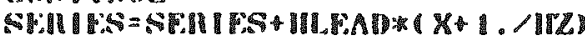

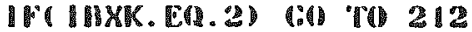

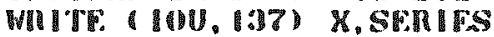

13

212

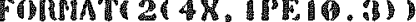

$x=x+8070$

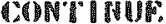

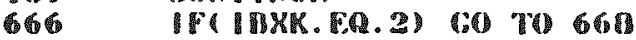

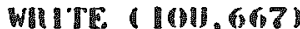

67

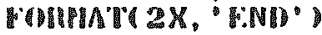

66 CONIINUP

(I)

SHULTOUT

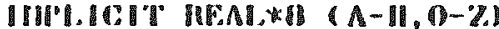

VI:ALER K

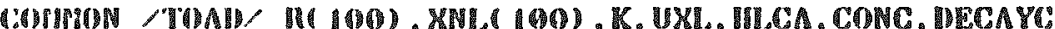

NTSHIN $=99$

$P=1$.

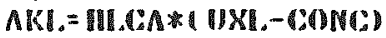

WIMT: $=X T^{\circ}$

SUINI $=0$

SUPH: $=0$

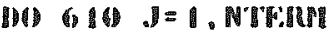

$\operatorname{li}: X=0$

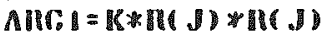

MIH:= AUV:I*THMH:

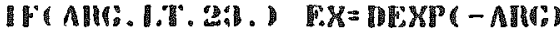

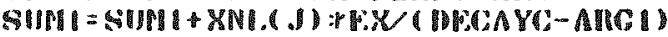

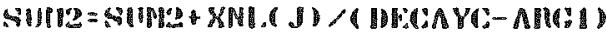

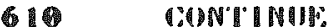

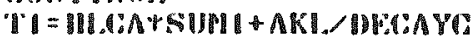

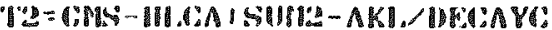

I: $x-0$.

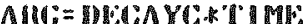

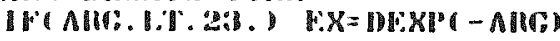

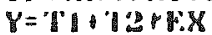

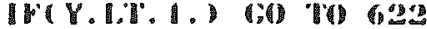

$6282 \pi=-1$

61: $1915=Y$ 


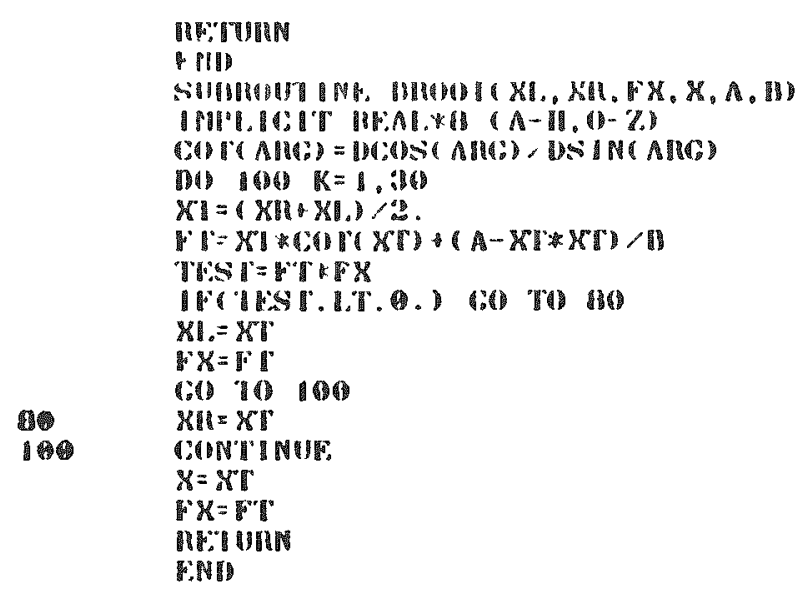




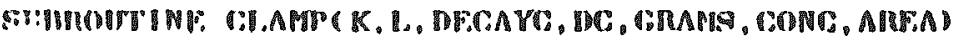

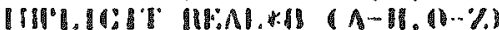

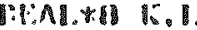

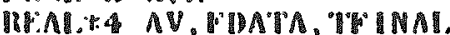

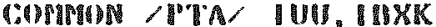

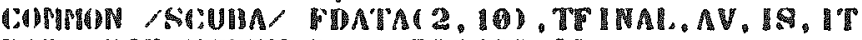

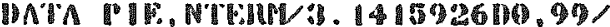

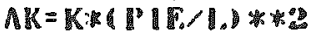

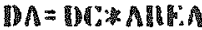

$T S=4.605 \% A M$

$P I=\mathbb{P} \| \mathbb{L}_{0}$

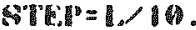

$X=1$.

$104=$ I UuU

IPIIIXK. Ma.2)

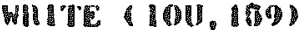

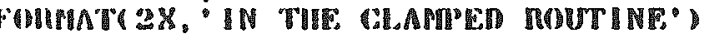

169

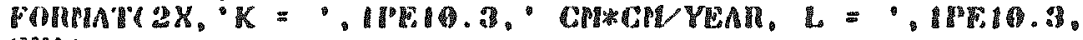

$1 \cdot 4 M^{\circ}$

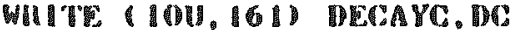

16

FOIMATI

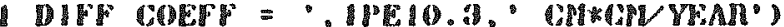

WIITR: (1011. 162) CONC

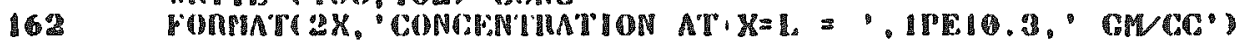

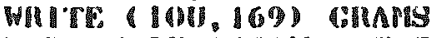

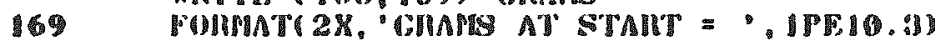

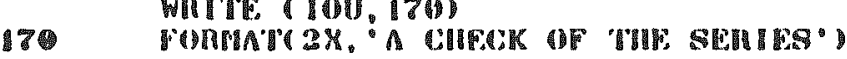

WIIIT: (1111, IT)

171

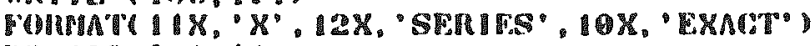

2 2은

$1=1,1$

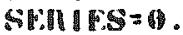

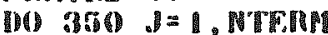

$A=\sqrt{1}$

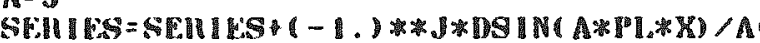

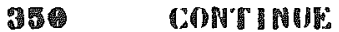

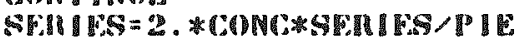

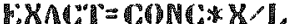

IFI IBXK. FR. 2$)$ CO 110202

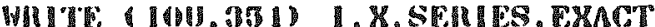

15

202

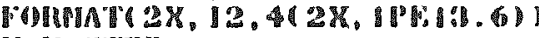

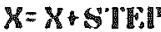

CONTTINUE:

I PI IMKK. R.2.2)

WIITR (IOU, 4OI) TS

놉항

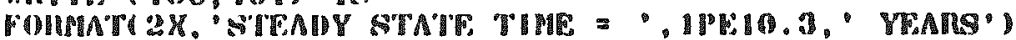

$205 \quad 16=1$

$X I=0$.

$\mathrm{XIN}=\mathrm{MS}$

$P=1$.

M) $100 \mathrm{KK}=1,89$

$X I=(X I 1+X I)+$,2 .

G:PBS = G:DLPMA

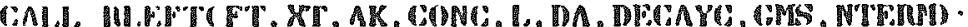

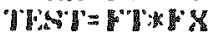

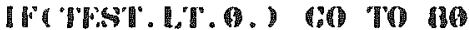

KI. $=81$

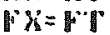

(i) 100

no

$\mathrm{XII}=\mathrm{XI}$

$15=2$

109

GIN

$I 1=1$

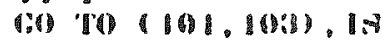

11

If 


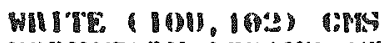

HOMIMT

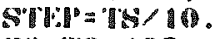

(2)

IC IIIKK. IOQ.2)

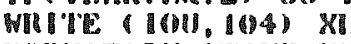

204

1 1ำ

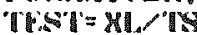

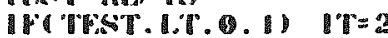

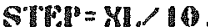

H IIIXK.

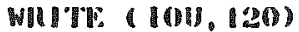

120

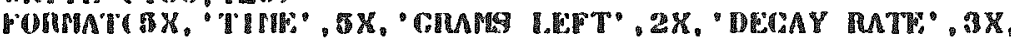

23

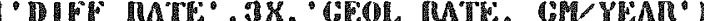

AK $=$ GODHG:

110) $109 \mathrm{KK}=1,10$

TV

SUPII $=0$.

SUPLO $=0$.

SIIPI: $=0$

Silpes $=0$

SUPB $=$.

SUMG $=0$

IOC $107=1$, NTE,EM

$x: X=0$

$A=d$

$A M I: 1=A K: A: A$

AMI:= AMB: $* T$ TINF.

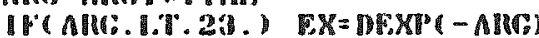

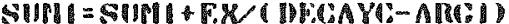

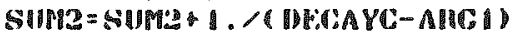

S11:N= $(-1) *$.

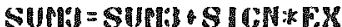

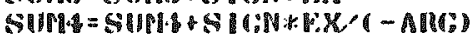

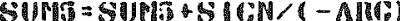

19

SUMG $=$ SUPG $+10 \%$

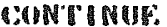

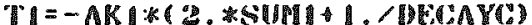

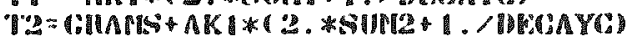

$F \cdot x=0$.

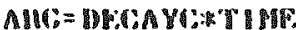

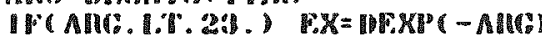

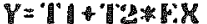

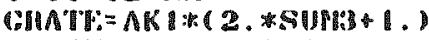

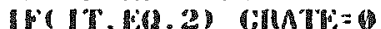

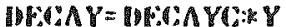

DIMTV $=A K I *(2 .+B U N G+1$.

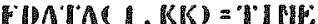

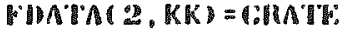

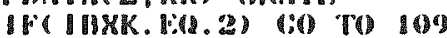

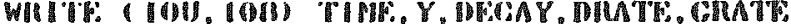

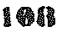

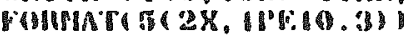

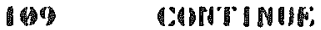

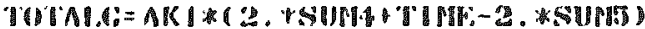

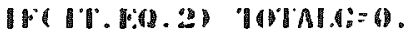

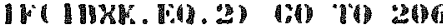

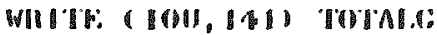

141

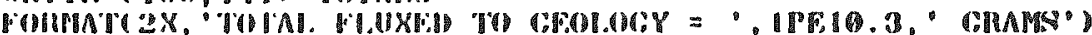

âto

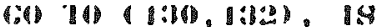

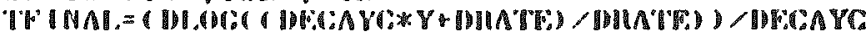

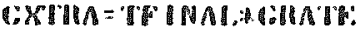

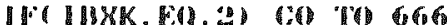

Iil

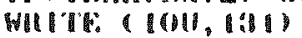

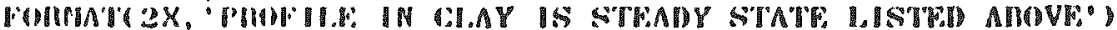

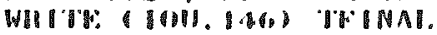




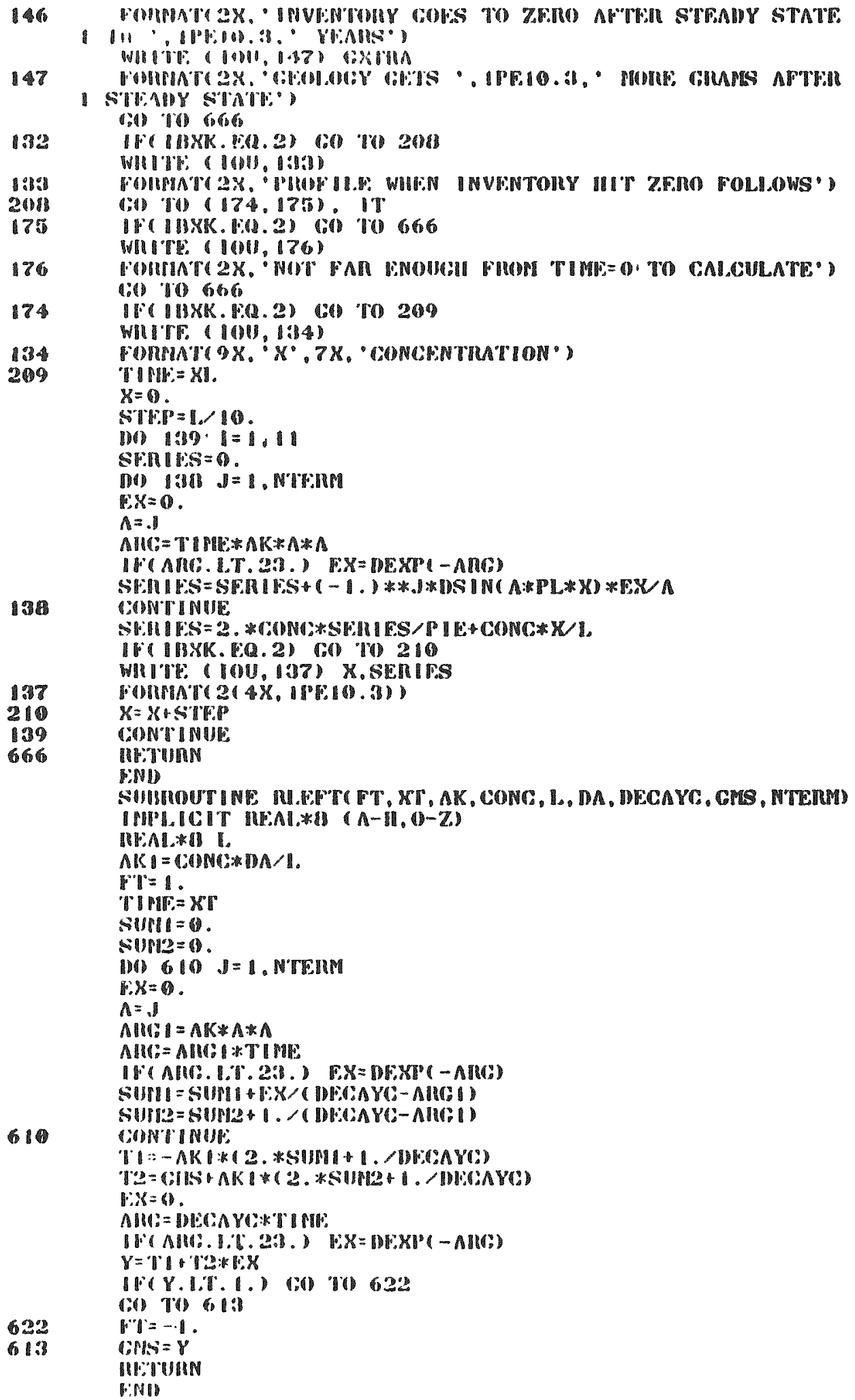


.

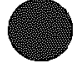


APPENDIX B

DATA BASE FOR CORROSION MODEL (CORODE)

Corrosion rates were determined on the basis of the following

(1) materials considered included oniy mild steel, Zircaloy-2, Inconel-600, 304SST, copper, lead and cast iron

(2) the barrier package was assumed to be filled with medium "Brine $B$ " or ground water of "low ionic strength" at one of two temperature ranges $\left(25^{\circ} \mathrm{C}-100^{\circ} \mathrm{C}\right.$ and $\left.100^{\circ} \mathrm{C}-250^{\circ} \mathrm{C}\right)$

(3) corrosion rates (mils/yr) were selected from open literature (Cheng, 1980)

(4) effects of irradiation and migration of chemical species were excluded

Tables $B-1$ and $B-2$ summarize the corrosion rates in mils/yr of metals under anoxic and oxic conditions immersed in Brine $B$ and ground water. Two types of corrosion rates were evaluated

(1) metal loss - steady corrosion rate calculated from descaled metal loss

(2) crack propagation - maximum crack penetration rate associated with pitting corrosion, stress corrosion or graphitization

The rationale of data selection for corrosion rates of package materials in Brine $B$ and ground water are discussed herein.

It was noted in (Braithwaite and Molecke, 1979) that solution corrosivity increases in the order: BRINE $B$ <Sea water <Brine $A$ for the barrier materials. Thus, the corrosion rates in Table B-1 taken from data in sea water 
Table B-1. Corrosion Rates of Barrier ilaterials in Brine B.

\begin{tabular}{|c|c|c|c|c|c|c|c|}
\hline \multirow[b]{2}{*}{ Materia! } & \multirow[b]{2}{*}{ Terrp. Range, ${ }^{\circ} \mathrm{C}$} & \multicolumn{3}{|r|}{$\begin{array}{l}\text { Mera] Loss } \\
(m i s / y r)\end{array}$} & \multicolumn{3}{|c|}{$\begin{array}{c}\text { Crack Propagation } \\
\text { (mils/yr) }\end{array}$} \\
\hline & & Anoxic & Oxis & Reference & Anoxic & Oxic & Reference \\
\hline \multirow[t]{3}{*}{ Mild Steel } & $25-100$ & 1 & - & $\begin{array}{l}\text { Posey Palko } \\
\text { Braithwite Molecke }\end{array}$ & $<k_{2} p$ & - & Braithwite Molecke \\
\hline & & - & 3 & schumacher & - & $27 p$ & Senumacner \\
\hline & $100-250$ & 2.8 & 276 & Braithwaite \& Molecke & «te & $<i g$ & Braithwate \& Molecke \\
\hline \multirow[t]{4}{*}{ Zircaloy-2 } & $25-100$ & $<\frac{1}{2}$ & - & Braithwate Molecke & $<\frac{3}{g} \vec{F}$ & <xp & Intemational Nicxel 60. \\
\hline & & - & $<\frac{1}{2}$ & International Nickel Co. & - & - & \\
\hline & $100-250$ & $<\frac{h}{2}$ & - & Braithwaite \& Holecke & $\sqrt{2} p$ & - & International Nickel Co. \\
\hline & & - & $<\frac{1}{8}$ & Intarnational Nickel Co. & $\cdot$ & $<b_{2} P$ & Braithwal:e \& Molecke \\
\hline \multirow[t]{2}{*}{ Inconel-600 } & $25-100$ & 1 & 4 & Reinnart & $5 p$ & $\operatorname{sip}$ & $\begin{array}{l}\text { intermational hekel co. } \\
\text { Schunather }\end{array}$ \\
\hline & $100-250$ & $<\frac{1}{8}$ & 4 & Braithwaite \& Molecke & $<\frac{1}{2} p$ & $<x p$ & Braitrwalte \& Molecke \\
\hline \multirow[t]{4}{*}{$30455 T$} & $25-100$ & ex & - & Todd \& Lovet: & 208 & - & Todd L Lover: \\
\hline & & - & 5 & Tuthill \& Senillmolar & - & 308 & Schunacher \\
\hline & $100-250$ & $<$ 装 & 3.9 & Braithwaite Molecke & $<\operatorname{tg} p$ & - & Braithaite Molecre \\
\hline & & - & - & & - & $3.7 \times 10^{4} \mathrm{~s}$ & Speide 1 \\
\hline \multirow[t]{2}{*}{ Copper } & $25-100$ & $<\frac{b}{y}$ & <h & Senumacher & $5 P$ & $5 P$ & Tuthill \& Senilinoler \\
\hline & $100-250$ & 3 & 87 & Braithwarte Molecke & $<x_{2} p$ & $\iota_{2} p$ & Braithwite \& Molecke \\
\hline \multirow[t]{2}{*}{ bead } & $25-100$ & Ch & 0.6 & Geel, et al. & <isp & $<z_{z}^{p}$ & Geel, et al. \\
\hline & $100-250$ & 12 & 47 & Braithwarte \& Molecke & $<\frac{3}{8}$ & $<s p$ & Sraitnwate \&olecxe \\
\hline \multirow[t]{4}{*}{ Gast iron } & $25-100$ & 3 & - & Senumacher & 128 & - & Hamer \\
\hline & & - & 54 & Harmer & - & 1006 & iuthill s Schllimoler \\
\hline & $100-250$ & 36 & - & Rabald & 408 & - & iutm \& Sen19:0 or \\
\hline & & - & 50 & Neison & - & 3000 & "utmili \& scmilimo er \\
\hline
\end{tabular}

(a) estimated from maximum crack penetration associated with ? (pitting corrosion, Sistress corrosion: or 6 (graonitization) daca. 
Table B-2. Corrosion Rates of Barrier Materials in Ground Water

\begin{tabular}{|c|c|c|c|c|c|c|c|}
\hline \multirow[b]{2}{*}{ Material } & \multirow[b]{2}{*}{ Tem. Range, ${ }^{\circ} \mathrm{C}$} & \multicolumn{3}{|c|}{$\begin{array}{l}\text { Metal Loss } \\
\text { (mils/yr) }\end{array}$} & \multicolumn{3}{|c|}{$\begin{array}{c}\text { Crack Propageron (a) } \\
(\text { m } 1 \text { (a) }\end{array}$} \\
\hline & & Anoxis & oxic & Reference & Anoxie & $0 \times 16$ & Reference \\
\hline \multirow[t]{2}{*}{ Mild Steel } & $25-100$ & 0.5 & 0.5 & Den?son Ronano & 29 & $2 P$ & Denison \& Romanoff \\
\hline & $100-250$ & $i$ & 2 & 8iazer Onens & $2 P$ & 199 & Cataldi cheng \\
\hline \multirow[t]{2}{*}{ Zirealoy-2 } & $25-100$ & $<\frac{2}{3}$ & sty & Berry & $\operatorname{sig} \theta$ & $<\frac{1}{2} P$ & Berpy \\
\hline & $100-250$ & $\sin$ & shy & Berry & $\operatorname{stg} p$ & $<\frac{4}{2} P$ & Berry \\
\hline \multirow[t]{3}{*}{ Inconel -600} & $25-100$ & $<\frac{2}{3}$ & $<$ 整 & Copson \& Berpy & $\operatorname{etg} p$ & $<$ şp & Copson \& Berry \\
\hline & $100-250$ & $<\frac{k}{2}$ & ex & Copson \& Berry & 235 & - & Builscheck Van Rooyen \\
\hline & & - & - & & - & $<\frac{1}{3} p$ & Copson Berpy \\
\hline \multirow[t]{4}{*}{$304 S 5 T$} & $25-100$ & $2 \frac{3}{8}$ & - & Dentson \& Romanoff & $\operatorname{crap}$ & $\infty$ & Denison \& Romanoft \\
\hline & & - & $<$ & Reinhart & - & $1.2 \times 10^{5} \mathrm{~s}$ & Ford \& Povich \\
\hline & $100-250$ & <年 & - & Copson Berry & $\operatorname{stg} p$ & - & Berpy \\
\hline & & - & $2 \frac{x}{y}$ & Catald \& Cheng & - & $3.8 \times 10^{6} \mathrm{~s}$ & Ford \& Povich \\
\hline \multirow[t]{4}{*}{ Copper } & $25-100$ & $<\frac{x}{2}$ & 92 & Syrett & <空P & - & Denison \& Romanoff \\
\hline & & - & - & & - & $<$ & Matison \& Fredriekson \\
\hline & $100-250$ & 2 & - & Hamer & $<$ sap & - & Hangmer \\
\hline & & - & 2 & Laque \& Copson & - & $<\frac{2}{3}$ & Laque : Copson \\
\hline \multirow[t]{2}{*}{ Lead } & $25-100$ & $<\frac{2}{3}$ & $<\bar{s}$ & Denison \& Romanoff & $<\frac{\sin }{8}$ & $0.6 P$ & Denison \& Romanoff \\
\hline & $100-250$ & $<\frac{1}{2}$ & <立 & Butler \& Ison & ip & IP & Anistrom \\
\hline \multirow[t]{3}{*}{ Cast Iron } & $25-100$ & 4 & $<\frac{2}{3}$ & Denison Romanoff & $35 P$ & $6 P$ & Denison \& Romanoff \\
\hline & $100-250$ & 2 & - & Hanmer & $8 P$ & - & Hamer \\
\hline & & - & 2 & Catald G Cheng & - & $35 p$ & Cataldr \& Cheng \\
\hline
\end{tabular}

(a) estimated from maximum crack penetration associated with $P$ (pitting corrosion), S(stress corrosion), or G (graphtization) data. 
or Brine $A$ for use in Brine $B$ can be considered to be conservatively high. For Brine $B$

(1) Mild Steel - Hot brines are very corrosive with corrosion rates increasing with brine velocity, oxygen concentration, temperature and other oxidation (Braithwaite and llolecke, 1979). In addition, mild steel does not usually pit severely or stress crack in hot solution. However, at low temperature oxygen tends to promote pitting. A corrosion rate equation (Posey and Palko, 1979) for metal loss in anoxic $4 \mathrm{M} \mathrm{NaCl}$ is in good agreement with (Braithwaite and Molecke, 1979).

(2) Zircaloy-2 - The corrosion rates in brine solutions are insignificantly low and the rates are not affected by the oxygen concentration and temperature range considered (Braithwaite and Molecke, 1979).

(3) Inconel-600 - The corrosion rates for metal loss are low and not affected by the oxygen concentration and temperature range considered (Braithwaite and Molecke, 1979). In addition, Inconel-600 is very resistant to chloride stress corrosion cracking (Schumacher, 1979). In anoxic solution at low temperature $\left(25^{\circ} \mathrm{C}-100^{\circ}\right)$, some pitting does occur (International Nickel Co.).

(4) Type 304 Stainless Steel - The corrosion rates for metal loss are much lower than for mild steel, but austenitic types are susceptible to pitting and stress corrosion. At low temperature $\left(25^{\circ} \mathrm{C}-100^{\circ} \mathrm{C}\right)$, oxygen concentration increases pitting rate while at high temperature $\left(100^{\circ} \mathrm{C}-250^{\circ} \mathrm{C}\right)$ oxygen concentration promotes stress corrosion cracking (Schumacher, 1979)(Todd and Lovett, 1956). (Speide1, 1977) reported a crack propagation rate of $3.7 \times 10^{4}$ mils/yr for sensitized type $304 \mathrm{~L}$ stainless steel in 42 percent ingCl at $130^{\circ} \mathrm{C}$. In view of the chloride concentration mechanism, the same order of nagnitude of crack propagation rate might be expected in oxic Brine $B$. 
(5) Copper - At low temperature, corrosion rates are small and relatively insensitive to oxygen concentration in slowly moving sea water (Schumacher, 1979). Highly oxygenated brines are corrosive with oxygen discharge usually the controlling factor (Braithwate and Molecke, 1979).

(6) Lead - The corrosion rates for metal loss increase with temperature and oxygen concentration (Braithwaite and Molecke, 1979).

(7) Cast Iron - The corrosion rates in crack propagation associated with graphitization mask the rates for metal loss. Oxygen significantly increases graphitization and leads to very high corrosion rates (Tuthill and Schillmoler, 1965).

Corrosion data taken from inorganic reducing acid (pH. 5.6) or oxidizing alkaline (pH 8.0) ground waters as well as hydrogen or oxygenated waters were used to estimate corrosion rates for metal loss and crack propagation associated with pitting corrosion and stress corrosion.

(1) Mild Steel - The corrosion rates for metal loss are small in anoxic and oxic water. At high temperature $\left(100^{\circ} \mathrm{C}-250^{\circ} \mathrm{C}\right)$ corrosion rate for crack propagation associated with pitting may be significant (Cataldi and Cheng, 1958).

(2) Zircaloy-2 - The corrosion rates are negligible in anoxic or oxic waters. No stress corrosion or pitting occurs (Berry, 1971).

(3) Inconel-600 - The corrosion rates are negligible in anoxic or oxic waters. No pitting or stress corrosion nomally occurs (Copson and Berry, 1960). Under certain sensitized conditions, stress corrosion cracking has been reported in pure deaerated water at high temperatures (Builischeck and Van Rooyen, 1980).

(4) Type 304 Stainiess Steel - The corrosion rates for metal loss are negligible in anoxic or oxic waters with no pitting or stress corrosion (Denison and Romanoff, 1946)(Copson and Berry, 1960). Under certain 
BWR water conditions, severe stress corrosion cracking of sensitized type 304 stainless steel can occur (Ford and Povich, 1979).

(5) Copper - The corrosion rates in anoxic or oxic water are insignificant. However, studies (Syrett, 1977) on the corrosion of copper in $30^{\circ} \mathrm{C}$ water contaminated with sulfide, oxygen, or both have shown that the presence of either sulfide plus low oxygen or oxygen alone causes low corrosions rates. If sulfide and oxygen are both present in certain concentration ranges, a dramatic increase in corrosion rate for metal loss may result. Pitting corrosion or stress corrosion is not likely in anoxic or oxic waters. Pitting of copper is usually a cold water phenomenon (Mattson and Fredrickson, 1968). Cold water pitting is associated with the formation of a protective mat of cuprous oxide on the copper surface (Powell and Lucey, 1966).

(6) Lead - Corrosion rates for metal loss or pitting in anoxic or oxic waters are negligible (Denison and Romanoff, 1946)(Butler and Ison, 1966).

(7) Cast Iron - The corrosion rates for metal loss are small in anoxic or oxic waters. However, high pitting corrosion rates have been reported both in reducing alkaline ground water $(\mathrm{pH} 7.1)$ and 10 w sulfide (Denison and Romanoff, 1946) and in high purity water with oxygen at $285^{\circ} \mathrm{C}$ (Cataidi and Cheng, 1958). 
APPENDIX C

DATA BASE FOR BARRIER FAILURE IMODEL (STRESS) CRITERIA

In the STRESS subroutine a number of material properties are used in the calculations. Properties were obtained from various sources and in some cases were estimated where no values could be obtained.

\section{Wall Materials - Compressive Yield}

Compressive yield strength is fitted as a linear function of temperature. Most values were obtained from the ASME Code Division 2, Section VIII (1977). These were usualiy available as a function of temperature. Some temperature functions were obtained by extrapolating data to a zero yield at the melting point. Because of the small temperature range of interest such approximations have only a minor effect on the end results.

Values for carbon steel specification SA-285 are given in Table C-1 and were taken from Table ACS-1 of the ASME Code. A linear fit with intercept $=15.7$ KSI and slope $-0.011 \mathrm{KSI} /{ }^{\circ} \mathrm{C}$ was obtained from these data.

Only one value for copper was available in Table ANF -2.2 of the code. For specification SB-11 copper the value reported is $10 \mathrm{KSI}$ at $20^{\circ} \mathrm{C}$. Extrapolation to zero yield at the meiting point of $1083^{\circ} \mathrm{C}$ gave an intercept of $10.2 \mathrm{KSI}$ and slopes of $-0.0094 \mathrm{KSI} /{ }^{\circ} \mathrm{C}$.

Inconel (Ni-Fe-Cr alloy $800 \mathrm{H}$ spec SB-409) data were then taken from the ASME Code and are given in Table C-2. A linear fit gave an intercept of $25.2 \mathrm{KSI}$ and slope of $-0.022 \mathrm{KSI}{ }^{\circ} \mathrm{C}$.

Values for Stainless Steel type $304 \mathrm{spec}$ SA-240 taken from the ASME Code are reported in Table C-3. A linear correlation gave an intercept of 30.7 $\mathrm{KSI}$ and slope of $-0.053 \mathrm{KSI} /{ }^{\circ} \mathrm{C}$.

The compressive yield strength used for cast iron was $20 \mathrm{KSI}$ at $20^{\circ} \mathrm{C}$ and was from the Mechanical Engineers Handbook (Marx, 1952). Extrapolation to zero at the melting point $\left(1538^{\circ} \mathrm{C}\right)$ gave an intercept of $20.3 \mathrm{KSI}$ and slope of $-0.013 \mathrm{KSI} / /^{\circ} \mathrm{C}$. 
Table C-1. Compressive Yield for Carbon Steel

(from ASME Div. 2, Section VIII, Table ACS-1)

\begin{tabular}{|c|c|}
\hline $\begin{array}{c}\text { Temperature } \\
(\mathrm{O} C)\end{array}$ & $\begin{array}{c}\text { Yield Strength } \\
(\mathrm{KSI})\end{array}$ \\
\hline 38 & 15.0 \\
93 & 14.6 \\
149 & 14.2 \\
204 & 13.7 \\
\hline
\end{tabular}

Table C-2. Compressive Yield for Inconel Alloy $800 \mathrm{H}$ (from ASME Div. 2, Section VIII, Table ANF-2.3)

\begin{tabular}{|c|c|}
\hline $\begin{array}{c}\text { Temperature } \\
(\mathrm{O} \mathrm{C})\end{array}$ & $\begin{array}{c}\text { Yield Strength } \\
(\mathrm{KSI})\end{array}$ \\
\hline 38 & 25.0 \\
93 & 23.1 \\
149 & 21.7 \\
204 & 20.3 \\
\hline
\end{tabular}


Table C-3. Compressive Yield for Stainless Steel Type 304 (from ASME Div. 2, Section VIII, Table AHA-2)

\begin{tabular}{|c|c|}
\hline $\begin{array}{c}\text { Temperature } \\
(\mathrm{OC})\end{array}$ & $\begin{array}{c}\text { Yield Strength } \\
(\mathrm{KSI})\end{array}$ \\
\hline 38 & 30.0 \\
93 & 25.1 \\
149 & 22.5 \\
204 & 20.8 \\
\hline
\end{tabular}

Table C-4. Material Properties (x $10^{3} \mathrm{KSI}$ ) (from Mechanical Engineers Handbook, by (Marks, 1952))

\begin{tabular}{|l|c|c|c|}
\hline \multicolumn{1}{|c|}{ Material } & Shear Modulus (G) & Bulk Modulus (B) & Poisson Ratio (V) \\
\hline Carbon Steel (rolled) & 11.3 & 20.2 & 0.265 \\
Cast Iron & 6.7 & 12.0 & 0.255 \\
Copper (annealed) & 5.8 & 17.9 & 0.355 \\
Inconel & 11.0 & - & - \\
Zircaloy & 4.8 & 3.9 & 0.44 \\
\hline
\end{tabular}


Similarly, the yield strength for Zircaloy was extrapolated from 43 KSI at $20^{\circ} \mathrm{C}$ (Marks, 1952) to zero at $1760^{\circ} \mathrm{C}$ for an intercept of $43.5 \mathrm{KSI}$ and slope of $-0.0247 \mathrm{KSI} / /^{\circ} \mathrm{C}$.

Wall Materials - Buik and Shear Modulus

Table C-4 gives values of bulk and shear moduli for various materials (Marks, 1952). Assuming a Poisson ratio, $v$, of 0.3 for inconel the bulk modulus, $B$, was estimated from the shear modulus, $G$, by

$$
B=\frac{2}{3} G\left(\frac{1+v}{1-2 v}\right)=\frac{2}{3}(11)\left(\frac{1.3}{.4}\right)=23.8 \times 10^{3} \mathrm{KSI}
$$

Extrapolation to zero at melting points shown in Table C -5 gave results shown in Table C-6 for the temperature fits of shear moduli.

Detailed data for stainless steel moduli were available (Datsko, 1966). The temperature fits developed for shear modulus had a slope of $-3.77 \mathrm{KSI} /{ }^{\circ} \mathrm{C}$ and an intercept of $10,000 \mathrm{KSI}$.

Bulk moduli are relatively insensitive to temperature and no reasonable basis for extrapolation was available. Stainless Steel data were available (Datsko, 1966). Table C-7 shows the temperature fits used for bulk modulus.

Wall Materials - Tensile Yield

The ASME code criteria for tensile yield were used in the STRESS subroutine. Wall thickness required is related to the allowable stress, $S$. The values of $S$ are obtained versus temperature from ASME, Division 1, Section ViII. Four coefficients are used to describe $S$ as a function of temperature that is 
Table C-5. Melting Points Used for Shear Modulus Extrapolation

\begin{tabular}{|l|c|}
\hline Material & Temperature $\left({ }^{\circ} \mathrm{C}\right)$ \\
\hline Carbon Steel & 1538 \\
Cast Iron & 1538 \\
Copper & 1083 \\
Inconel & 1455 \\
Zircaloy & 1760 \\
\hline
\end{tabular}

Table C-6. Temperature Fits for Shear Modulus

\begin{tabular}{|l|c|c|}
\hline \multicolumn{1}{|c|}{ Material } & Intercept (KSI) & Slope (KSI/OC) \\
\hline Carbon Steel & $5.84 \times 10^{3}$ & -3.8 \\
Cast Iron & $6.78 \times 10^{3}$ & -4.4 \\
Copper & $5.9 \times 10^{3}$ & -5.5 \\
Inconel & $11.2 \times 10^{3}$ & -7.67 \\
Zircaloy & $4.88 \times 10^{3}$ & -2.77 \\
\hline
\end{tabular}


Table C-7. Temperature Fits for Bulk Modulus

\begin{tabular}{|l|c|c|}
\hline Material & Intercept (KSI) & Slope (KSI/OC) \\
\hline Carbon Steel & 17,900 & 0 \\
Cast Iron & 12,000 & 0 \\
Copper & 17,900 & 0 \\
Inconel & 23,800 & 0 \\
Zircaloy & 3,861 & 0 \\
Stainless Steel & 19,310 & 1.05 \\
\hline
\end{tabular}

Table C-8. Allowable Stresses for Internal Pressure

\begin{tabular}{|l|c|c|c|c|}
\hline Material & $\begin{array}{c}S I \\
(P S I)\end{array}$ & $\begin{array}{c}S 2 \\
(P S I / O C)\end{array}$ & $\begin{array}{c}S 3 \\
\left({ }^{\circ} \mathrm{C}\right)\end{array}$ & $\begin{array}{c}S 4 \\
(P S I)\end{array}$ \\
\hline Carbon Steel & 12500 & 0 & 50 & 12500 \\
Zircaloy & 13451 & 26.7 & 38 & 12436 \\
Inconel & 21200 & 0 & 50 & 21200 \\
$304 S S T$ & 19316 & 16.3 & 38 & 18700 \\
Copper & 7399 & 19.5 & 38 & 6660 \\
Cast Iron & 6000 & 0 & 50 & 6000 \\
\hline
\end{tabular}




$$
\begin{array}{ll}
S=S 1-S 2(T) & T>S 3 \\
S=S 4 & T \leq S 3
\end{array}
$$

(C.2)

where $T={ }^{\circ} \mathrm{C}$ and $\mathrm{S}=$ PSI.

The values are summarized in Table $C-8$.

Note that for materials for which $S$ is independent of $T$, an artificial temperature break was introduced to satisfy the logic in the code.

Backfill Materials - Bulk Modulus

The pressure-volume relationship of a packed granular material was represented by

$$
P_{R}=A\left(\frac{V_{0}}{V}-1\right)+k\left(\frac{V_{0}}{V}-1\right)^{2}
$$

where $\quad V_{0}=$ original volume, $\left(\mathrm{cm}^{3}\right)$

$V=$ volume after compression by $P_{R}\left(\mathrm{~cm}^{3}\right)$

$$
\begin{aligned}
& P_{R}=\text { external pressure, (KSI) } \\
& \left.\begin{array}{l}
A \\
K
\end{array}\right\}=\text { empirical constants, (KSI) }
\end{aligned}
$$

The bulk modulus was obtained by

$$
B=A\left(V^{*}+1\right)+2 K V^{*}\left(V^{*}+1\right)
$$

where

$$
V *=\left(\frac{V_{0}}{V}-1\right)=\frac{-A+\sqrt{A^{2}+4 K P_{B}}}{2 K} \text { or } \frac{P_{R}}{A} \text { if } K=0
$$

$C-7$ 
A typical soft sand in the literature (Byerlee, 1967 ) gave values of $A=0.44 \mathrm{KSI}$ and $K=253 \mathrm{KSI}$.

A stiff sand modeled after a powder compaction model was also considered (Cooper and Eaton, 1962). This gave values of $A=47.5 \mathrm{KSI}$ and $K=0$ KSI. Balues of $A$ and $K$ were input and the value of $B$ was calculated by the code.

Poisson Ratio and Mohr Slope - Backfill Material

The shear and stress distribution properties of the backfill were characterized by a Hohr circle slope, $\beta$ which relates yield and imposed external pressure, and the Poisson ration, $v$, which relates bulk and shear moduli. Data for high fractured rock or concrete with low porosity (i. e., the fragments are fitted together rather than jumbled) suggest a value of $\beta=1.2$ and Poisson ration of 0.25 to 0.4 (Byerlee, 1967). Pre-compacted fine sand or sand/clay mixtures indicate that values would be more in the range $\beta=0.6$ and $0.35 \leq v \leq 0.45$.

For the backfills considered in this study the appropriate values were $\beta=0.6$ and $\nu=0.4$. 
APPENDIX D

INPUT/OUTPUT DESCRIPTION

This appendix is designed to guide the user through the steps necessary to operate the BARIER code. First, a description of the input data required to operate the code is presented. Then, the input techniques and requirements for execution are discussed including operator-machine interactions and procedures. Finally, a description of output data and format is presented.

\section{D.1 INPUT DATA}

All non-internal physical data required to execute BARIER are contained in five external data files:

(1) CORRAT - contains corrosion rate data for use in the CORODE subroutine for each possible package material (metal) as a function of temperature and water type (corrosive environment)

(2) GEOMAT - contains repository physical data for each of the four geologies of concern (salt, shale, granite, basal $t$ )

(3) MATMAT - contains all material stress and other related constants utilized in the STRESS subroutine

(4) BARFIL - a dummy variable for a file containing all necessary package design data or specifications for an individual case to be evaluated - each individual case has its own separate file name 
(5) DATSET - durmy variable representing a file listing all "BARFIL" files to be run

The corrosion rate data contained in CORODE is comprised of eight separate values for each package material (metals). Four corrosive environments are considered:
(1) Anoxic brine $B$
(2) Oxic brine B
(3) Anoxic water
(4) Oxic water

over two tenperature ranges $\left(25^{\circ}-100^{\circ} \mathrm{C}, 100^{\circ}-250^{\circ} \mathrm{C}\right)$. Each corrosion rate is assumed constant over its temperature range and is taken from the maximum of rates corresponding to specific corrosion mechanisms. A listing of CORRAT with current data is provided in Appendix $E$.

The repository physical data for the four geologies is contained in GEOPAT and includes the variables shown in Table $0-1$. The format of GEOMAT is $(A 5, F 10.0, A 5,6 F 10.0)$ and a listing with current data is provided in Appendix E.

Physical constants utilized in the STRESS subroutine are contained in IATMAT and include the variables shown in Table 0-2. The format of MATMAT is (10F10.0) and a listing with current data is provided in Appendix E.

For each specific package design case to be evaluated by BARIER, a BARFIL data file must be provided. BARFIL is actually a dumny variable name equivalent to a specific file name corresponding to a specific package desijn. A complete physical description of the specific package design is supplied to BARIER by this file. The variables included in BARFIL are shown in Table D-3. A value for each of the variables in Table $0-3$ is suoplied for each barrier of a particular package design. The format of BARFIL is (5X, I5, 7F10.0, /, 215, $2 F 10.0,215,2 F 10.0,2 F 5.0)$ and a sample listing is provided in appendix $E$.

For each radionuclide of concern, input data to the RELEAS subroutine are stored in an array NUCLID $(I, j)$. The $i$-th radionuclide is 
Table D-1. GEOMAT Variables

\begin{tabular}{|c|c|}
\hline Variable & Definition \\
\hline LABEL & - Geology \\
\hline REPRES & - Repository pressure, psia \\
\hline CREEP & - Signifies creeping geology (yes or no) \\
\hline EGEO & - Emissivity of repository surface \\
\hline$T 1)$ & - \\
\hline $\begin{array}{l}T 2 \\
T 3\end{array}$ & $\begin{array}{l}\text { - Constants used in repository temperature } \\
\text { correlations }\end{array}$ \\
\hline T4 & - \\
\hline COEFF & $\begin{array}{l}\text { - Overa } 11 \text { heat transfer coefficient between } \\
\text { repository and barrier, } \mathrm{w} / \mathrm{in}^{2} . \mathrm{O}_{\mathrm{K}}\end{array}$ \\
\hline
\end{tabular}


Table D-2. MATMAT Variables

Varjable

Definition

Y1 - Coefficient for yield strength temperature correlation, Ksi

Y2 - Coefficient for yield strength temperature correlation, $\mathrm{Ksi} /{ }^{\circ} \mathrm{C}$

81 - Coefficient for bulk modulus temperature correlation, Ksi

B2 - Coefficient for bulk modulus temperature correlation, Ksi/ ${ }^{\circ} \mathrm{C}$

G1 - Coefficient for shear modulus temperature correlation, Ksi

G2 - Correlation for shear modulus temperature correlation, $\mathrm{Ksi} /{ }^{\circ} \mathrm{C}$

s1 - Hoop stress yield temperature correlation constant, psi

S2 - Hoop stress yield temperature correlation constant, psi/ ${ }^{\circ} \mathrm{C}$

S3 - Hoop stress yield temperature correlation constant, ${ }^{\circ} \mathrm{C}$

s4 - Hoop stress yield temperature correlation constant, psi 
Table D-3. BARFIL Variabies

\section{Variable Definition}

IL - Type of barrier (stabilizer, can, overpack, sleeve)

IDIAM - Inside diameter of inner barrier layer, in.

ODIAM - Outside diameter of inner barrier layer, in.

ODIAM2 - Outside diameter of second innermost barrier layer, in.

FDIAM - Outside diameter of third barrier layer (backfill), in.

GDIAM - Outside diameter of outer barrier layer (gap), in.

A - Backfill pressure-volume coefficient, psi

K - Backfill pressure-volume coefficient, psi

MAT - Type of material in inner barrier layer

MAT2 - Type of material in second innermost barrier layer

E - Joint efficiency in stress calculation, dimensionless

COAT - Coating delay time (for corrosion), yr

BAK - Backfill material type

MATGAP - Gap material type

CLPRES - Internal pressure on barrier at time of repository sealing, psi

CLTEMP - Internal temperature on barrier at time of repository sealing,

BETA - Backfill Mohr circle slope, dimensioniess

Poiss - Backfill Poisson ratio, dimensionless 


$$
\begin{aligned}
i=1 & : \text { Uranium-238 (high solubility) } \\
2 & \text { : Uranium-238 (low solubility) } \\
3 & \text { : Plutonium-239 } \\
& \text { 4: Americium-241 }
\end{aligned}
$$

The columns of IUCLID $(I, J)$ contain the following radionuclide specific information:

$$
\begin{aligned}
j=1 & : \text { grams at } t=0 \\
2 & : \text { conversion factor for grams to curies } \\
3 & : \text { concentration, } \mathrm{gm} / \mathrm{ml} \\
4 & : \lambda \text { in, yr-1 } \\
5 & : \text { diffusion coefficient (liquid), } \mathrm{cm}^{2} / \text { year } \\
& 6: \mathrm{k}_{\mathrm{d}}, \mathrm{ml} / \mathrm{gm}
\end{aligned}
$$

Columns 7 - 10 are zeroed out but available for use. Use is made of column 10

\begin{tabular}{|c|c|c|c|c|}
\hline & $Q_{i}$ & $\lambda$ & $k_{d}$ & $c$ \\
\hline$i=1$ & 471420 & $1.5 \times 10^{-10}$ & 50 & $1.07 \times 10^{-3}$ \\
\hline 2 & 471420 & $1.5 \times 10^{-10}$ & 1800 & $3.3 \times 10^{-11}$ \\
\hline 3 & 2953 & $2.8 \times 10^{-5}$ & 1200 & $3.8 \times 10^{-11}$ \\
\hline 4 & 539 & $1.5 \times 10^{-3}$ & 4000 & $3.8 \times 10^{-11}$ \\
\hline
\end{tabular}
where the radionuclide quantity at some time $t>0$ is stored.

The value of $D$ used in the RELEAS calculations is that for a substance in water and is conservatively estimated to be $10^{-6} \mathrm{~cm} / \mathrm{sec}$ or $31.5 \mathrm{~cm}^{2} /$ year (Smith, 1970). However, the value of D used to calculate $H$ is 0.10 to account for the fact that the corroded barrier has a decreased diffusivity due to void volume and tortuosity. The other input data for all radionuclides are

$$
\begin{aligned}
\varepsilon & =0.01 \text { to } 0.1 \\
\delta & =4 \\
\rho & =1 \text { to } 2 \mathrm{gm} / \mathrm{ml}
\end{aligned}
$$

The radionuclide specific data are the initial radionuclide quantities, $u_{i}$, tne equilitorium constants, $k_{d}$, and the concentrations, $c$. These data are 
The concentration estimates were obtained from the experimental value for plutonium (Katayama, 1976), except for the uranium-high concentration yalue which approximates that of the uranyl carbonate complex (Neretnieks, 1978). The $k_{d}$ values were obtained from the same reference for the uranyl carbonate complex solubility, except for the case of uranium-high solubility which was conservatively set at 50. Since americium-241 is a decay product, an initial quantity for it was calculated so that the quantity at large time (i.e., after the parent has decayed) would be correct if the parent were not transported out of the fuel bundle. This results in a conservative initial quantity for americium-241 because the parent (plutonium) does transport out of the fuel bundle.

\section{D.2 INPUT TECHNIQUES AND REQUIREMENTS}

The BARIER code as written is tailored for a time-shared terminal but oniy minor modifications would be required to allow batch processing. Input is made through a series of input files and some control parameters obtained by interrogation on the terminal. A driver file must be prepared listing the BARFIL files by name for each package design case to be evaluated. This driver file has the dummy variable name DATSET in the program and can be given any valid name which is entered on the terminal when requested. The general form of DATSET is shown in Table D-4 where each BARFIL file is identified (e.g., A.1) along with the corresponding number of barriers in that particular package design case. The format for DATSET is (A10, 12). Thus, an unlimited number of independent and consecutive package design cases may be evaluated with one input message.

Upon execution of BARIER, the input information shown in Table D-5 is requested by the terminal (in order) and typed in by the user. Execution of the program may be terminated by entering "STOP" when the program requests a new driver file. 
Table D-4. General Form of DATSET.

\begin{tabular}{|lc|}
\hline FILE & NUMBER OF BARRIERS \\
A.1 & 2 \\
A.2 & 3 \\
A.3 & 2 \\
B.1 & 3 \\
B.2 & 3 \\
CI.1 & 3 \\
E.1 & 4 \\
\end{tabular}


Table D-5. Input Information Required for BARIER Execution

(1) Name of the driver file containing the list of BARFIL files

(2) Time increment (DELTA) by which time will be varied when performing successive calculations leading to barrier failure, yrs

(3) Geology code (IGE), (1-salt, 2-basalt, 3-granite, 4-shale)

(4) Water code (IWATER), (1-anoxic brine, 3-anoxic water)

NOTE: The code increments IWATER so that both anoxic and oxic cases are automatically run. 
All output from BARIER is stored in a data file called PERFOR.DAT. Output for all cases listed in DATSET is maintained in this file until a subsequent execution of BARIER with a different DATSET file. Each time a DATSET file is evaluated by BARIER, output from the previous program execution is overwritten in PERFOR.DAT. Output printouts may be obtained by writing PERFOR.DAT following termination of BARIER execution.

Output from a sample problem is provided in Appendix E. For each design case, pertinent input data is printed first and is followed by specific performance data for each type of environment (water) to be analyzed. Starting with the outermost package barrier, data at the time of failure of each barrier is printed. This includes barrier failure time, net pressure on the barrier at time of failure, barrier thickness, and temperature, repository temperature, and radiation dose. Leach begin time, or the time of failure of the innermost barrier, is printed next and is followed by nuclide geology release rate information from the RELEAS subroutine. For each package design case and water environment (brine or water) specified, BARIER anaiyses are performed for both the anoxic and oxic environments and are printed separately in the output on successive pages.

The output data from the RELEAS subroutine are the radionuclide release rates as a function of time at the backfill-geology interface as defined in Equations $(3.5 .1)-(3.5 .4)$. There are three types of release output data possible:

(1) When there is a sufficient quantity of a radionuclide at the beginning of the leach time tor each steady state transport, the output will be ten release values for $0.1 t_{s}, 0.2 t_{s}$, etc., where $t_{s}$ is the time to reach steady state. The release rate at steady state is the value reported at $t_{5}$. The time is then printed for the initial quantity of radionuclide to decay and diffuse away until none remains.

(2) When there is not a sufficient quantity of the radionuclide at the beginning of the leach time to reach steady state, the tine when the quantity goes to zero is divided by ten and release rates are reported for $0.1 t, 0.2 t$, etc. 
(3a) When there is not a sufficient quantity of the radionuclide at the beginning of the leach time to transport to $0.1 t_{s}$, no release rates are reported because the concentration profile at the backfill-geology interface is too flat for all times.

(3b) When there is less than one gram of radionuclide at the start of the release calculation, the calculation is not performed.

In order to write only the data described above, the write switch IBYK must be set to 2. Setting $I B Y K=1$ will result in the writing of intermediate results. This option is available to aid in determining which radionuclide quantities are important in the transport rates. In order to obtain a printout of the fourier series coefficients and roots as defined in Equation (3.5.20), the write switch IP must be set to 1 and $I B Y K=1$. These two switches are not external input and must be set through a statement or DATA block. 


\section{APPENDIX E \\ SAMPLE PROBLEHS}

Examples of sample problems and BARIER input data are provided in this Appendix. Files GEOMAT.DAT, CORRAT.DAT, and MATIMT.DAT contain indut data required to run any and 211 package design cases. File CoMllorl.HEd is included throughout BARIER and is shown here for completeness. Files D2.1, BE.27N, and E.11N represent particular barrier package designs (BARF IL file) which are evaluated by 3 ARIER.

In these sample problems, a time increment of 1.0 year, a salt geology, and an anoxic and oxic brine will be the remaining input control variables. This information is entered on a remote terminal upon request. Output for these cases is also presented. 
GEMTH TAT

\begin{tabular}{|c|c|c|c|c|c|c|c|c|}
\hline 8AT T & 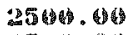 & YI & 0.75 & 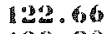 & \$1 100 & 50.010 & 145.04 & 5.60 \\
\hline MASA & 4506.94 & No & Den & 1.38 .880 & 11.13 & 10.90 & 200.00 & 5.00 \\
\hline GHAII & 5006.10 & Nol & 18 & 120.34 & 28.98 & 100 & 190.00 & 560 \\
\hline WIIAI & 210606 & YI & 0.75 & 160.456 & : & 15 & 108.00 & 5.60 \\
\hline
\end{tabular}

coHUนT . DA

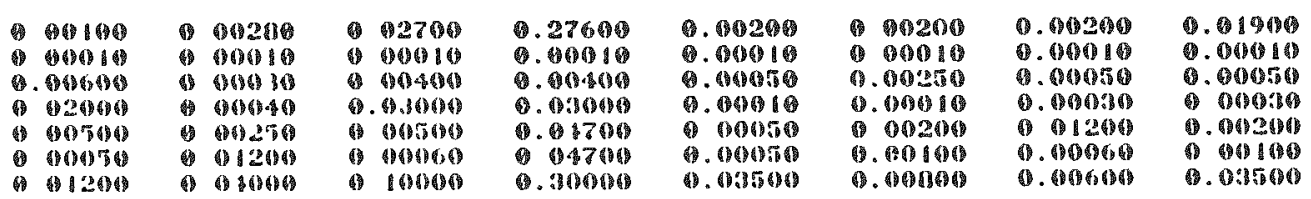

MATMAT. MAT

\begin{tabular}{|c|c|c|c|c|c|c|c|c|c|}
\hline $\begin{array}{r}15.7 \\
43.5 \\
25.2 \\
36.7 \\
10.2 \\
6.0 \\
36.0\end{array}$ & 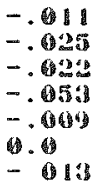 & $\begin{array}{r}17903 . \\
11361 . \\
311346 . \\
19310 \\
17906 . \\
13960 .\end{array}$ & $\begin{array}{l}0.00 \\
1.00 \\
1.000 \\
1.05 \\
0.00 \\
0.00 \\
0.00\end{array}$ & $\begin{array}{r}58340 . \\
4801 . \\
11200 . \\
16900 . \\
5900 . \\
0700 .\end{array}$ & 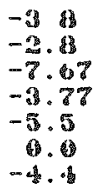 & $\begin{array}{r}12500 . \\
15351 . \\
31200 . \\
19316 . \\
7390 . \\
0 . \\
6000 .\end{array}$ & $\begin{array}{r}6.0 \\
8.9 \\
9.0 \\
16.3 \\
19.5 \\
0.6 \\
0.0\end{array}$ & $\begin{array}{l}50 . \\
318 . \\
50 . \\
38 . \\
818 . \\
5 . \\
58 .\end{array}$ & $\begin{array}{r}12500 . \\
12436 . \\
31396 . \\
10790 . \\
6660 . \\
0000 .\end{array}$ \\
\hline
\end{tabular}

DOMMON. NH

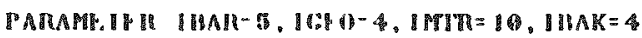

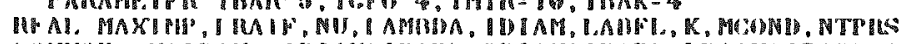

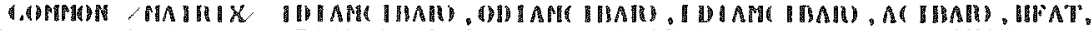

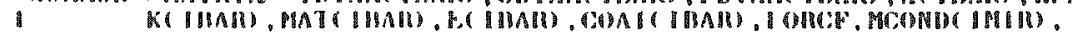

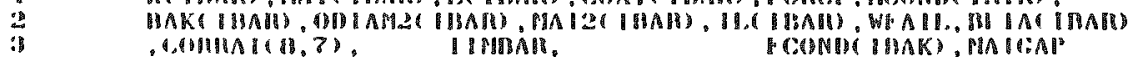

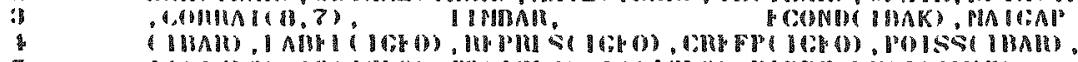

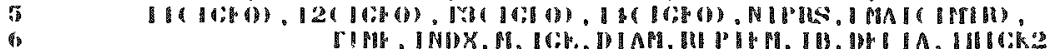

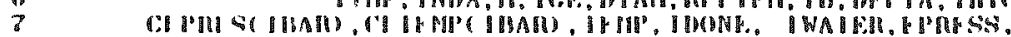

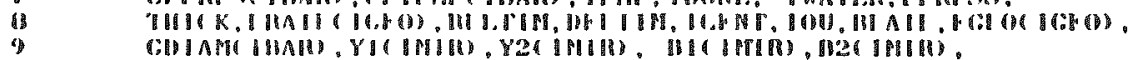

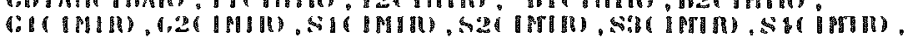

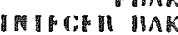

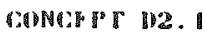

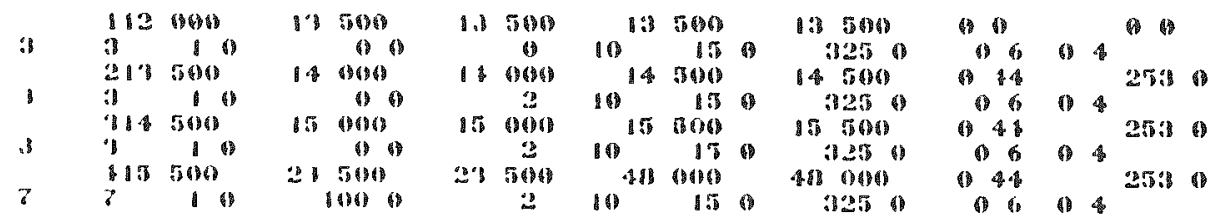




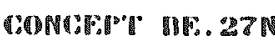

\begin{tabular}{|c|c|c|c|c|c|c|c|c|}
\hline & (1) 12.0000 & 198.80 & 18.600 & 19.50101 & 15.6040 & 0.0 & & 0.0 \\
\hline 6 & 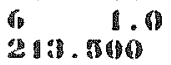 & $14.0000^{\circ}$ & 14.030 & 10 19.800 & 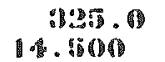 & $\begin{array}{l}\text { D. } 6 \\
0.414\end{array}$ & 0.0 & \\
\hline$A$ & 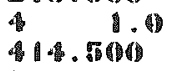 & 21.700 & $\$ 1.0100$ & (1) & $\begin{array}{l}1850.010 \\
410.000\end{array}$ & 0.0 & 0.4 & \\
\hline 8 & 1.1 & 0 & 1 & 0.0 & 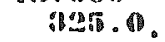 & 0.6 & 0.4 & \\
\hline
\end{tabular}

Concrib'

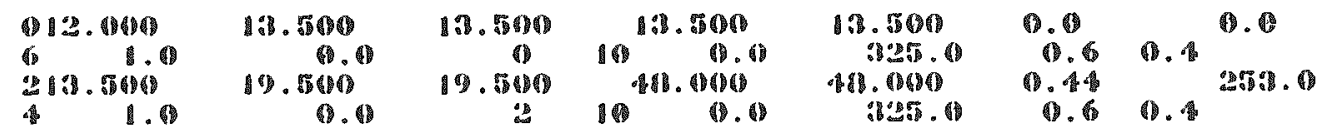




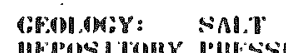

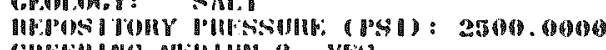

(a)

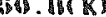

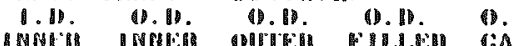

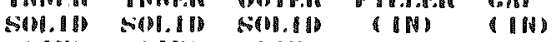

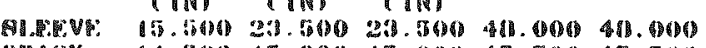

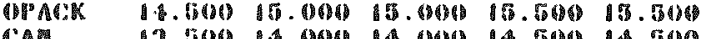

GAM

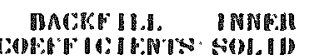

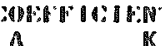

GIIT)
Sigh 1 B

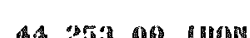

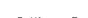

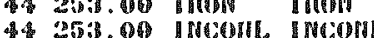

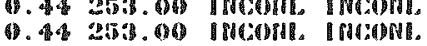

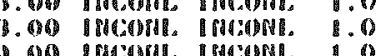

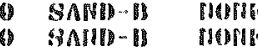

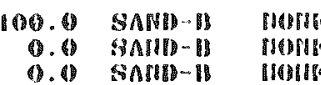

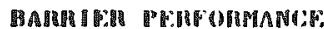

\begin{tabular}{|c|c|c|c|c|c|c|}
\hline \multirow{2}{*}{ 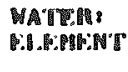 } & 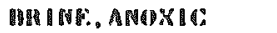 & & & & & \\
\hline & FALIUMP: & NEST & 'Hinckubss & ELIPAPRTI & HEDPSITYOBY & IMAD IBOSG: \\
\hline & ThPige mist & 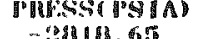 & $(1 \mathrm{NB}$ & TWPBS" KR & 'W: WIS' $\mathbb{K}\}$ & TINIII \\
\hline 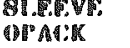 & $\begin{array}{l}19)^{2} .060 \\
5068.006\end{array}$ & $\begin{array}{r}-58183.65 \\
-3528.0 .8\end{array}$ & $\begin{array}{l}0.1800 \\
0.171\end{array}$ & $\begin{array}{l}4680.811 \\
469.07\end{array}$ & $\begin{array}{l}166.008 \\
666.00\end{array}$ & 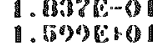 \\
\hline ring & 301.000 & - 258 & 0.1860 & \pm 609.30 & 866.00 & 2. 83.366060 \\
\hline
\end{tabular}

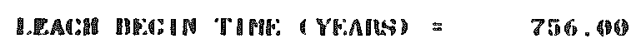

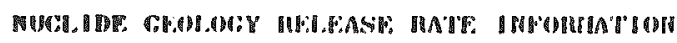

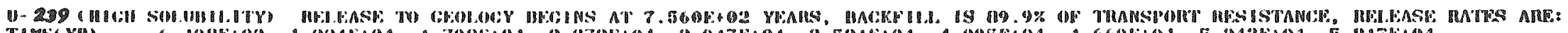

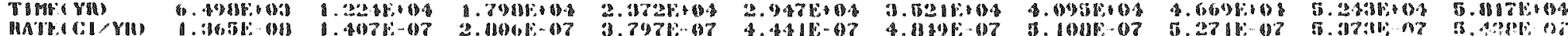
comeraper

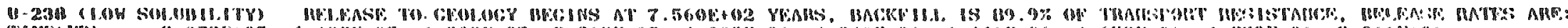
TIMEC YII

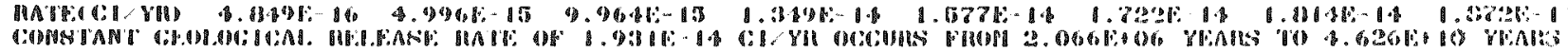

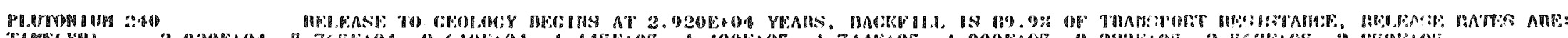

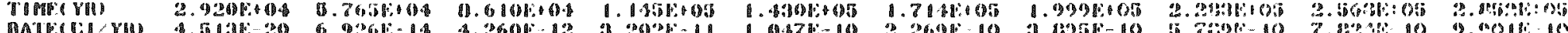

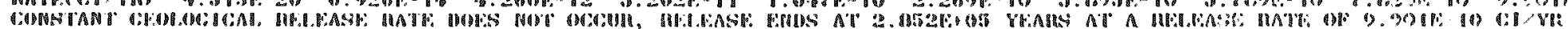

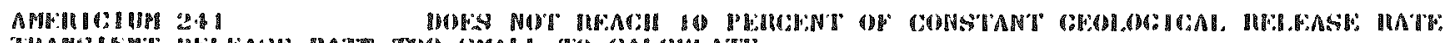

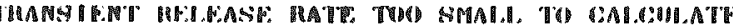


Concept D2.1 (Continued)

\begin{tabular}{|c|c|c|c|c|c|c|}
\hline WATHAL: & HOUNE.01KU: & & & & & \\
\hline RID.MP:NT & 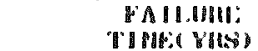 & 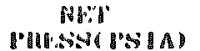 & 'H lokniss & 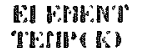 & 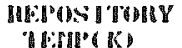 & MAB BOSB: \\
\hline sil.t & 1101.648 & -27609.4318 & 00.1000 & 46011.116 & $4 x_{0} 60.900$ & 1. 118080000 \\
\hline$B B^{2} A$ & 40.00 & -2928.91 & 0.170 & 460.07 & 96060.00 & $3.1908+838$ \\
\hline GAN & 311.06 & -96034.52 & 0.150 & 469.836 & 86060.000 & $1.72001 ;+6018$ \\
\hline
\end{tabular}

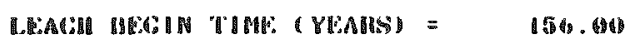

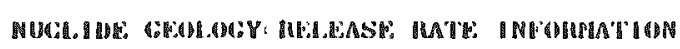

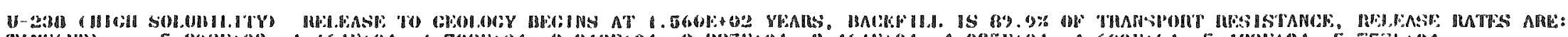

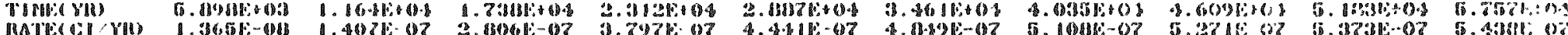

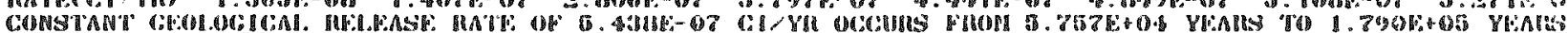

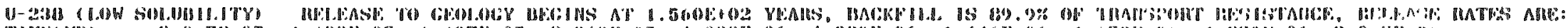

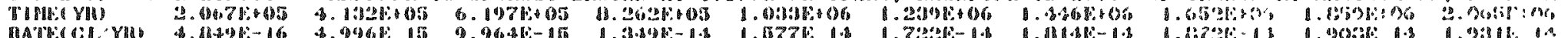

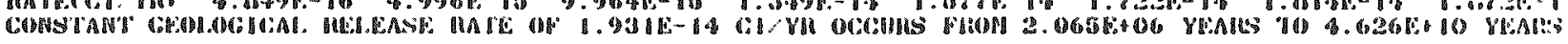

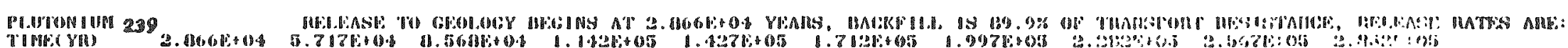

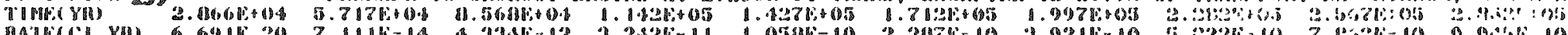
MA T.

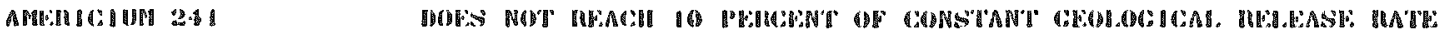

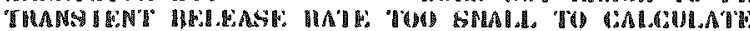


GHOH.OB:Y: SNAT

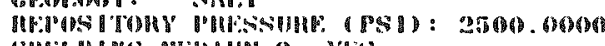

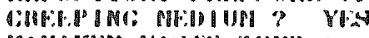

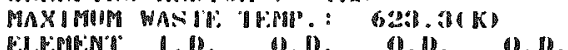

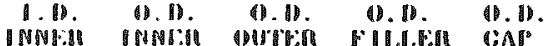

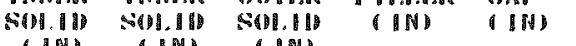

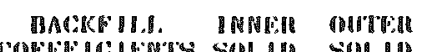

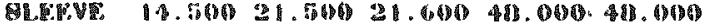

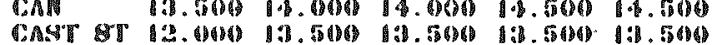

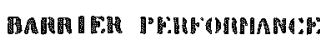

\begin{tabular}{|c|c|c|c|c|c|c|}
\hline 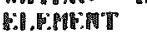 & FA HI. He? & MEIO & IUH C:KMHS: & FEMAMPNT & 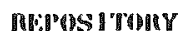 & IRAI MOSPי, \\
\hline & THPr: YALS & 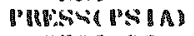 & (1) & Thise' (K) & Twair R & (I) Ism) \\
\hline ก.T.K. & 501898.006 & :อ57600. & 0.6000 & 469.14 & 486.069 & $1.47006-03$ \\
\hline EAN & 638.060 & 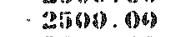 & 9.6000 & 469.018 & 468.000 & A. $1366080 \%$ \\
\hline CANH MPA & $6,13.000$ & . & O.6un & 460.8609 & 406.00 & 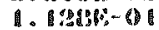 \\
\hline
\end{tabular}

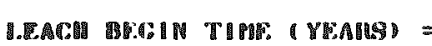

1378.00

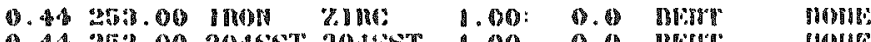

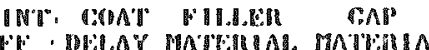

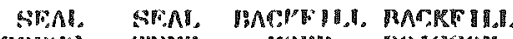

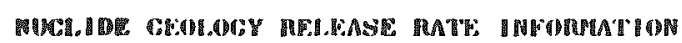

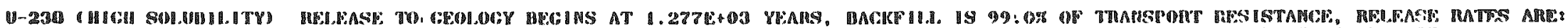
TIPEC YYH

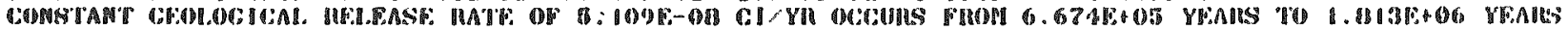

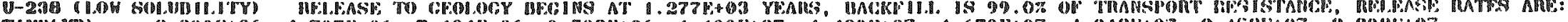
THPEC YMU

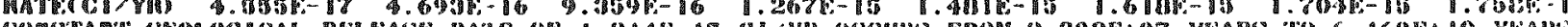

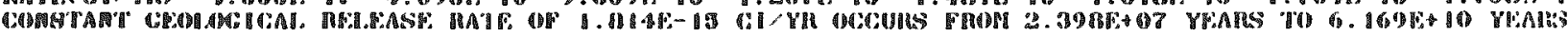

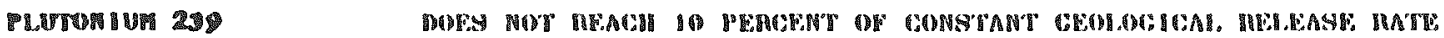

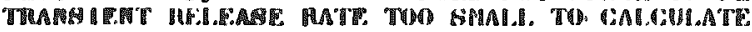

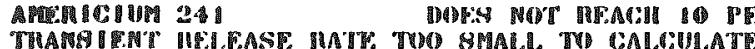


Concept BC.27il (Continued)

\begin{tabular}{|c|c|c|c|c|c|c|}
\hline $\begin{array}{l}\text { WATHAR: } \\
\text { WHA }\end{array}$ & MEUNA, OBXI: & & & & & \\
\hline 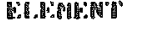 & Falloble: & Promision & 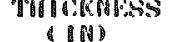 & 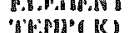 & If.PI) & 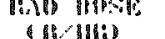 \\
\hline SIIFVE & 8712.000 & -85800.000 & 0.00001 & .669 .126 & 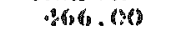 & $8.0616 \cdot 0): 8$ \\
\hline GAN & 9.00 & -35800.040 & 0.0480 & 460.917 & 9866.0180 & 3.20761009 \\
\hline COAST GMA & 16.000 & -2500.00 & 0.000 & 469.78 & 8460.000 & 2. 6010 tion \\
\hline
\end{tabular}

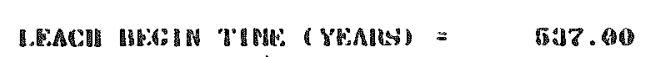

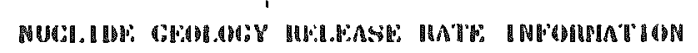

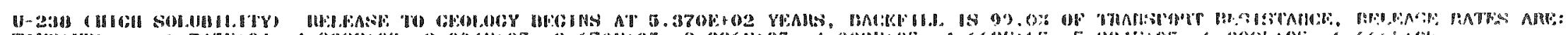
TIME YUB

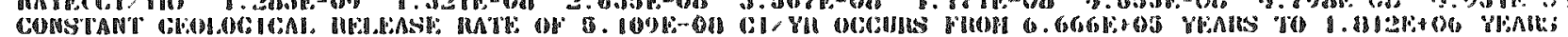

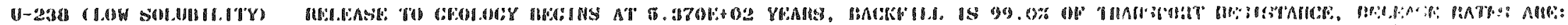

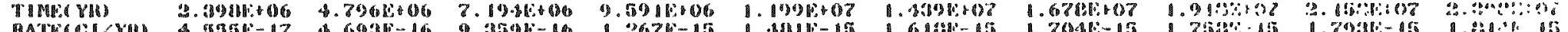

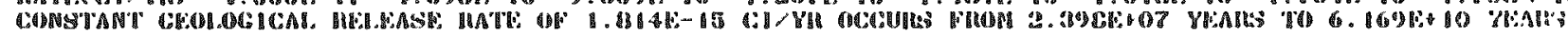

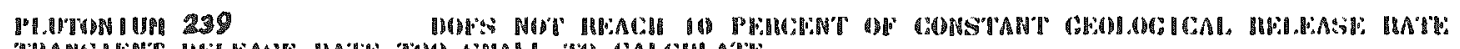

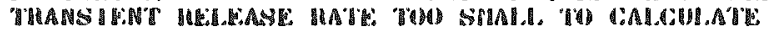

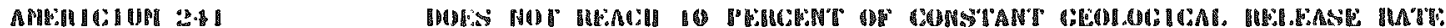

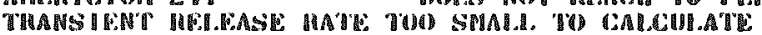


GROLABOY: SALT

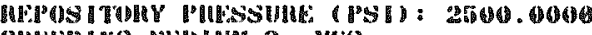

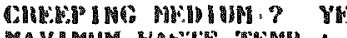

MAXIMUM WAS'TE TREAP"

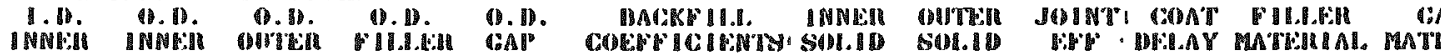

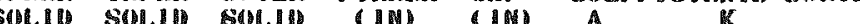

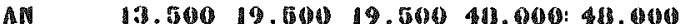

CA8T ST'

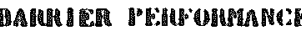

\begin{tabular}{|c|c|c|c|c|c|c|}
\hline 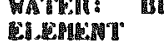 & 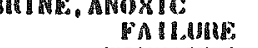 & NOS & THI ICKNESS & RLEOAENT & 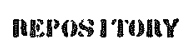 & MAD DOSE. \\
\hline $\begin{array}{l}\text { GAP } \\
\text { GABT ETAR }\end{array}$ & $\begin{array}{c}\text { THEC Yuss } \\
7500.00 \\
62.00\end{array}$ & 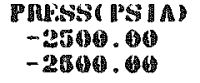 & $\begin{array}{l}100 \\
0.000 \\
0.000\end{array}$ & $\begin{array}{l}\text { THPHP I K } \\
469.104 \\
469.00\end{array}$ & $\begin{array}{r}\text { TEPAP } \\
466.00 \\
466.00\end{array}$ & 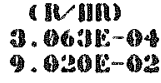 \\
\hline
\end{tabular}

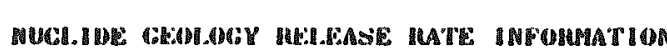

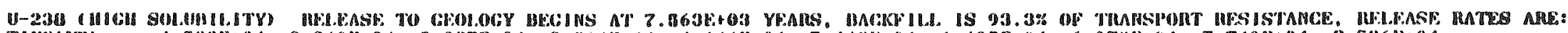
TIMEC YHA

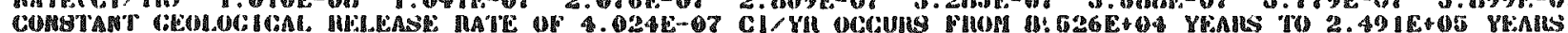

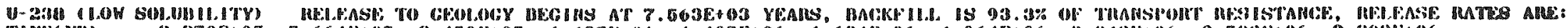
TIMECYIB

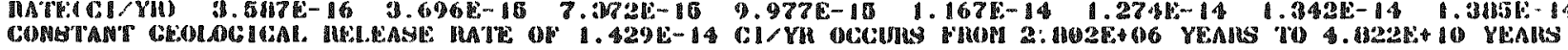

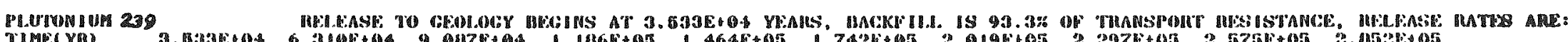
TIMEC YM

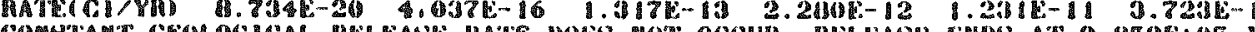

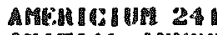

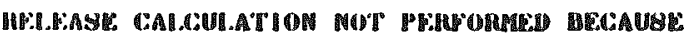

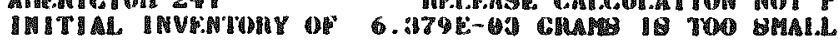


Concept F.llid (Continued)

\begin{tabular}{|c|c|c|c|c|c|c|}
\hline MATE:H: & 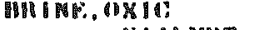 & & & & & \\
\hline 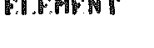 & 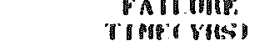 & 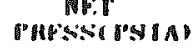 & $\begin{array}{l}\text { THGKNBSS } \\
\text { RMI }\end{array}$ & 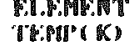 & 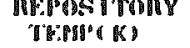 & 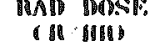 \\
\hline G:AFB & 100000800 & $\sin 6060$ & 0.07008 & 869.86 & 4606.070 & C. A.BABOOOOOO \\
\hline Gist AInB & 180.0800 & 2860600.4808 & 0.00000 & 400.97 & .90000 .090 & 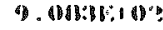 \\
\hline
\end{tabular}

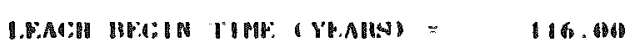

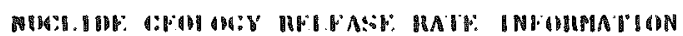

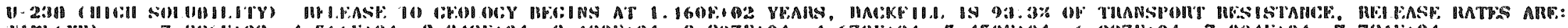

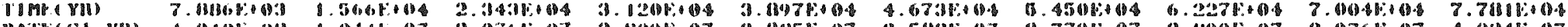

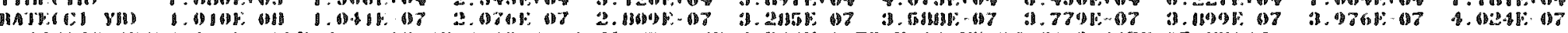

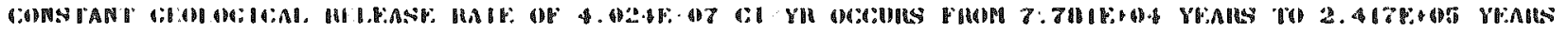

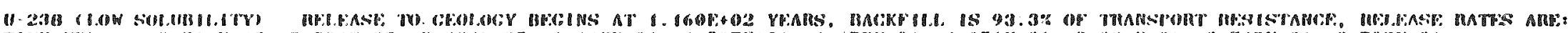
TIME: YID (DONO

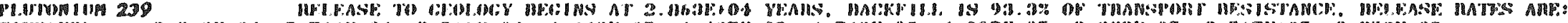

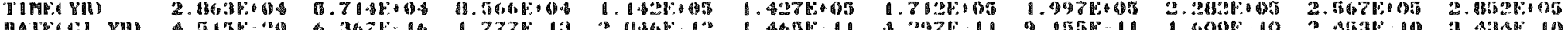

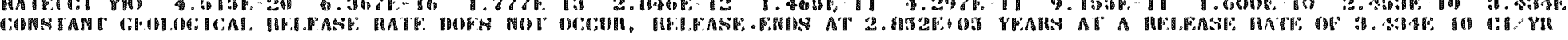

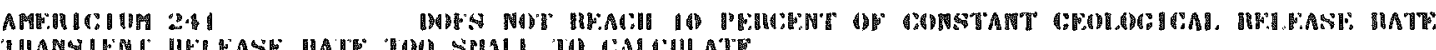


-

○ 


\section{DISTRIRUTION LIST}

ACEES AMERICAN INC

A \& PURCLSS

R STRUBLF

ALLIRD GENERAL NUCLEAR SERVICES

PI HICHBERGER

A A KOLB

ALLIS-CHALMERS

GARRICKI SOIOBE

AMERICAN NUCLEAR INSURERS

DOTIIF SHERMAN

ANALYSIS \& TEMNOLOGY INC I MAZOUR

ANALYTC \& COMPUTATIONAL MESEARCH

INC

B SAC.AK

APPLIED MECHANICS INC

JOHAR MILLIAMG

ARGONNE NATIONAL LABORATOYY

1 HOW ARD NITILL

W I MFCHAM

MARTIN SHITL

MARTINISTEINDLEP

MENC RESEARCH CORP

H P HIATLER

ARTHUR D. LITTLE INC

CHARIESR HADLOCK

ATOMIC ENERGY CONTIOL MOARD

$I$ L $\mathrm{M} \triangle \mathrm{ACH}$

ATOMC ENERGY OF CANAOA LTD

$\$ 1 O 11 \mathrm{KE}$

$A \cap \triangle Q L I N$

F PARGLAT

ATOMIC ENEEG RESEARCH ESTARLSSHMENT

D P HODGKINSO

IOH R A

ATOMIC INDUSTERAL FOIUUM INC

AUSTRALIAN ATOMIC ENERGY COMMISSION

BABCOCK \& WLCOX

WFORAATION SERUIE

BATTELL COLUMBUS DUVISIOA

SAFORDG BIOOM

IOHNT MCGNAT

IFFRI \& I QAFA

NEII F AULLER

TTPHES NKOLOS

IHONASM TR IIALR

KE $\ E H R$ Y ITS

BECHTEL GROUP INC

HOMAS'BAIR

DOA B GRADLAL

IFGIIE I JARDINF

$\checkmark$ A MORAIA

RICHARD I TOSEIII

WFNDIX FILD FNGINEERING CORP

IOHAC PACER

BHABHA ATOMIC RESEA RCH CEATRE

SL MOR MOR

K T THOMAS

BIACK \& VEATCH

M IOI ROROBINSON

RRITISH NUCLEAR FUELS LTD

$R \&$ MILS

PROOKHAVEN NATIONAL LABORATORY

GIRAIDBIDA

DOAALDI CLARK

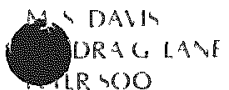

HILN IODOSOM 12

RUNDESMINISTERIUM FUR FORSCHUNG

ROLF-PFTER R AMDI

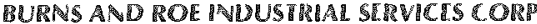
IOH PIRRO

C.P.H.F.

BHII DI ISIAC

C. W. WATIS ASSOCHATES

CURTIS $11 T$ S

CALPORNIA DEPT OE COASERVATION PLRR A $\triangle 1$ IITOS

CALIFOPNIA DEPY OF HEALTH SEMUICES BEIFRICT BIYR

CALFORNIA INSTITUTE OF TRCHNOLOGY LEOY $T$ SULVR

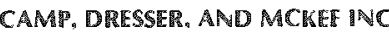

DAYLA A MOODKLIF

CAPITAL AREA GROUND WATER

CONSERYATHON COMMISSIOA

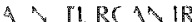

CASTUS VALLY ORCHARLS

CARL $A$ DRRSON

CAYUGA LAKE CONSERVATION

ASSOCHATION INC

OS KIEFER

CENTRAL WASHIAGTON UNUVRSITY

IR HI"VIHORNI

CENTRE D ETUDE DE: LENEEGE NUCLEAIRE RFPU HEREAIMS

CENTRE DINFORMATIQUE GEOLOGIOUE GHISL II DE MARSILY

CTIZENS ASSOCHATON FOY SOUND ENERGY

11 \& $1 \mathrm{~T}: 1 \mathrm{LI}$

COLUMBIA UNIVERSTY

HI BTRT OT I DIG ${ }^{5}$

CONVERSE WARE

$\checkmark$ S HALC

CORNELL UNIVERSITY

IOHS. BIRD

COUNCIL ON FCONOMIC PIIORTIES $\triangle 1 R$ I Y RESNIKOFF

CVGNA ENEGY SERVICES

DONAIDGARDIR

DAMES MOOME

$R O A, A T A R$

O L ORIL VULI

DAPPOLONIA CONSULTING ENGIEERS INC ISA D DO OHLI

ABBY JORRE S

AIIR I HASIOY

PETIR KHSAL

CARLE GRHLBLI

DAWCOA MANAGEMENT CONSULTING

SEIVICE

DAIIU A WEETIR

TBSLWARE CUSTOA MATEPEEL INC

HOMARD $\triangle O M T R$

DEPT OR THE INVIRONMENT

$F \& H E T T S$

DIVISON OF INVIRONMEATAL SAFET

RESEARCH

HARLTO AKALRA

DRAVO ENGINEERS AND CONSTRUCTORS RCD BLAIL

DUKE UNIVESTT

THOAIA DINW

DYMATECH RT COAPAN

GIIPHE $\mathrm{F}$ QITTH

EI. DU PONT DF NEMOURS \& COMPANY

DON THOT CORDON

E.R.IOHNSON ASSOCIATES INC

I R IOHThOS

(1) $10 \mathrm{HA}$ ax

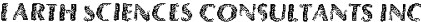
HARRY $L$ CROL $5 E$

RBASCO SERVICES INC

CI BAIR SALLM

CDS NUCLFA INC

( I GURAR VI.

EC \& G IDAMO INAC

GLORG B B IFUM

$\triangle D$ MCCORMACK

THSN1IH

RIC IIRD IALLAA

ELECTROWATT EAGIMEERING SERVICE HNPBTH

ELSAM

A $>10411$

EMPASSY OF THE UNITED STAIES

VAALIIIHAUIIA-RYIANDIR

ENERCOR INC

IOHA ROOOMIII H

EARGY FUELS NUCLEAR INC

DON $\triangle 1$ PILLMORE

ENERGY RESEARCH GROUP INC

MAR GOLDSAITH

ENERY RESEARCH LABORATORY MTACHI INC

DIAKOIOKIRL $6 \mathrm{HH}$

ENVIRONMENT CANADA

CLALDL BARRALD

ENVIRONMENTAL POLCY NSTITUTE DAUID A BPRIK $K$

TRED AILL $A R$

ENVIRONMENTAL RESEARCH GROUP INC PIILB G COLIN

ENVIROSPHETE TOMPANY $B O B$ HARDS

EXXOA NUCLEAR IDAHO COMPAVY INC D L COVDOTT

I $B$ KLR

EXYON RODOCIOA RESEARCH

GARI WAYIRL

FENIX \& SCISSON INC

IOSF A NAC HADO

CHARLEYI SBARK IA

FLORIDA INSTITUTE OF TECHNOLOG 1OSLPH A A YGELOIR

FLUOR ADVANCED TECHNOLOGU DUVISION JOA $D$ A C IRRY

FLUOR ENGINERS \& CONSTRUCIORS INC RAIAOAD I DIGAI

FORD, OACON \& DAVIS UTAHINC

PRESTOAH HI ATER ROBERTF OUERMIR BLRIOYI THA DIFR

FOSTER-MILLER ASSOCIATES INC YORBERT PAAS

FREIE UNIVERSTAET BEMUIN

HASKARI BRI LHI

GENEMAL ATOMIC COMPANY ROPERI \&I BI RGOY IF

ROBCRT I A APAAA

GENERAL COURT OF MASSACHUSETTS

TINOHHY I BLRKI

GEOLOGICAL SURVEY OF CANADA ROON 350

GEORGIA INSTITUTE ON TECHNOLOGY

GEHFLY LUCHHOL

AIIRD SCHNDDER

GEOTRONS

IASIES AILRCIR

GERMANTOWN FRENDS SCHOOI

IIIRB BASGOM 
GIBBS \& HLL INC

ROBERT PRIETO

GLBERT/COMMONWFALTH

JLRRY ILLIS

GOLDER ASSOCIATES

FLITABETH EISENHOOD

CIEAIFAT H $\mathrm{H}$ HLA

GTC GEOLOGIC TESTING CONSULTANTS LTD IOHAF PICKESS

H \& RECHNICAL ASSOCIATESINC

WIIIARIR RHYAL

HAHN-MEITNER-INSTITUT FUR

GERAFORSCMUNG BERLN

KI $A L C C A R I$ MAASS

HANFORD ENGINEERING DEVELOPMENT

LABORATORY

ALBERT BI BSFWIL

ROBERT EIVIIGER

R L KNECHII

W F ROAKE

HARDING LAWSON ASSOCIATES

FRAAk C RRLSSL

HARVARD UNIVERSITY

RAYATOVD GIEUER

IDAHO BUREAU OF MINES A DO GEOLOGY

[ARI H BEMVTI

IMPERIAL COLLGE OF SCIENCE AND

TECHNOLOGY

$B K 4 T K I N G O R$

INSTITUT FUR TIEFLAGERUNG

WERNT BRIWIT7

ALALSKLHN

I R SOLTER

PITER LERPM ANA

INSTITUTE TOR CHEAICAL TECHNOLOGY

RIINHARD ODOI

RESTITUTE OF GEOLOGICAL SCIENCES

VEII A CIIAPMAN

INSTITUTE OF RADIATION PROTECTION

$K A 114 K O B G S O N$

INTERA ENVRONAE WAL CONSULTANTS INC

I PEARSO VIR

ROBERT WILIAS

INTERNATIONAL ENERGI SYSTEAS CORP

JOHY B BOWLS

INTERNATIONAL ENGINEERING COMPANY INC

TERRY L STEIREORA

MA Z TSIAWSKI

INTERNATIONAL RESEARCH AND

EVALUATION

R DANFORD

IIT CORP

STOKES

ISTITUTO SPERIMENTALE MODELLI

STRUTTURE S.P.A.

F GERA

1.F.T. ACAPITO \& ASSOCIATES INC

WICIALLP HARDY

IACKSON STATE UNIVERSITY

ESTLS SMITH

IAPAN ATOMIC ENERGY RESEARCH INSTTUTE TARO 170

IGC CORPORATION

MASAHKO MAKMNO

IOINT RESEARCH CENTRE

GIRARDI HRANCESCO

IORDAN CORMILL ASSOCIATES

JOHN D THWHEY

KAISER ENGINEERS INC

n ) DODSO

I $\$$ RITCHIE
KANSAS DETT OF HEALTH AND

ENVIRONMENT

GERAIDIV ALLV

XIS

I ARSB Q VI SSON

WELLR WREATH ASSOCIATES

IRA Y W WEATH

KERNFORSCHUNGSZENTRUM KARLSRUHE GMBH

K O ClOSG

R KOSTLR

HURST PENTIVGHALS

WHN ASSOCIATES

HIRRY AHW

KLM ENGINEERING INC

B CEORGE KNALWYCI

KYOTO UNIVERSTY

YORITERU INOI F

LAWRENCE ERKELEY LABORATORY

$1 O H A$ A $1 P P 4$

THONASDO

YORATAN 1 EOELSTEN

BRIAAK ANHIRO

- MIVIR

RUBI SPFACER

I $\mathrm{AACG}$

LAWRENCE LIVERMORE NATIONAL

LABORATORY

LKADCA B BALLOL

IOHNH CAAPBELI

D D JAKGOV

R CARROIL MANACER

I AMRE YCID RAMASPIT:3,

U C Y TCLPE

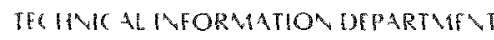
$\mathrm{L}-53$

RICHARD I A RONYNENBERC,

IEHIGH UNVVESTTY

[) $R$ SIMPYON

LOS ALAMOS NATIONAL LABORATORY

FPALT A BRY AVI

GIORGEA CONAN

BRI C ER TRDIL

CIMDIIERRICK

k $k \& P \| I A\}$

KL RI WOLISBFRC

LOS ALAMOS TECHNHCAL ASSOCIATES INC

R I KIMCSBLRY

LOUISIANA TECH UNIVERSTYY

IIBRARY

YOR IAA WITRIAI

MACLAREN PLANSEARCH INC

VIEX BLCHУFA

MASSACHUSETTS INSTITUTE OF

TECHNOLOG

IOHA OKT TCH

RICHARDK LETER

MIRGHAITUINF

MCDEMMOTI INC

KARLVL IURLON

MCMASTER UNIVERSITY

I W YHENILT

MECHANICAL TECHNOLOGYINC

U IRRE,$B E S Y L E R$

STANET M DOROFF

MEMBERS OF THE GENERAL PURLC

UAVIOH BOITZ

IAILSBOHD

WLUAML CONAWAY

WILIAM: CONN

DP DALTOVICH

DAENELL O DLDER

FRACEST ARITY

SHIRIIY M GIFORD
DOLGISH GREPIE

(I IANTK

T) C LAAGSTAIR

D 11101910

11+ MCDOMHL

AI SO PASTERA AK

SHAIIERS PHIIBRICK

ROCFEI POUERS

PAL SHEWALOA

11 I SZI IINAI

IIMRY I MHTE

MICHAEL BARER, JR INC

( I TOLHILI

MICHIGAN DEPT OF PUBLC HEALTH

DON 1 A $\square$ AROME

MICHIGAN DISTRICT HEALTH DEPT NO

UDG, SRRET

MICHIGAN LEGISLATIV OFFICE OF SCIENCE

ADVISOR

MICHIGAN TECHNOLOGICAL UNVERSTY

CARY I DOW VEY

MINNRSOTA GEOLOGICAL SURVEY

MATT \& UITOY

MISSISSIPPI ATTORNEY GENERALS OFHICE

MARCAMEROV

MISSISSIPPI CITIZENS ACAINST NUCLEAR DISPOSAL

TAVIEYOHA TIINT

MISSISSIPP DEPT OF ENERG AND

TRANSPORTATION

IOHE W GRERN:3

MISSISSIPPI DEPT OF NATURAL MESOURCES

(HARIFSI BLALOCK

MISSISSIPPI DEPT OF WIDLIFE

CONSERVATION

IOSEPH II ICOB IR

MISSISSIPPI STATE ROARD OF HEALTH

CDOEL 5 TESTF

I UARRIN GREF

MBSISSIPPI STATE HOUSE OT

REPRESENTATIVES

JERRY ORIEFE

MITRE CORP

LESTER A ETTIMUER

MITSUBISHI METAL CORP

TATSLIO ARIMA

VASA IOHNSON SPACE CENTTE

WICHALL R HELERT

NATIONAL ACADEMY OF SCIENCES

IOHNT HOHLOM

PETFR D VIFES

NATIONAL BOARD FOR SPENT NUCLEAR

FUEL, KARNBRANSEENAMDEN

MII $~ R Y D E L L$

NATIONAL BUREAU OF STANDARDS

RILYM $M$ CHYO

IVILLA I P RLLO

NATIONALE GENOSSENSCHAFT PUR DIE

LAGERUNG RADIOAKTIVER AREALL

I IRLIESRLHV

NATURAL RESOURCES DEFENSE COUNCIL

THONASB COCHR IN

NEVADA DEPT OF ENERGY

ROBFRTR LOL X

NEW ENGLAND NUCLEAR CORP

KERRY BLYNLRT

NEW JERSEY DEPT OE ENVIRONMENTAL

PROTECTION

IF A NETTE EM

NEW MEXICO ENVIRONMENTAL EVALUAT GROUP

ROBFRT H WIL 
NEW YORK DEPT OF HEALTH

$$
\begin{aligned}
& \text { DAY XFLRODMD } \\
& \text { NEW STATE ELECTRIC \& GAS CORP }
\end{aligned}
$$

LFWISE STALY

NEW YORK STATE ENVIRONMENTAL

FACILITIES CORP

PICKFTT T SINPSON

NEW YORK STATE ERDA

JOHR C DE SIPSFY

NEW YORK STATE GEOLOGICAL SURVEY

ROBERT H FAKL ADIVY

NEW YORK STATE PUBLIC SERVICE

COMMISSION

FRED HAAC

NEW YORK UNIVERSITY MEDICAL CENTER

MLRRIL FISENBL D

NORTH DAKOTA GEOLOGICAL SURVEY

DON 1 HAIVORSOS

NTR GOVERNMENT SERVICES

THOMAS! RLY VOLDS

NUCLEAR ASSURANCE CORP

IOHN \ HOI STOA

RHONNEL SAIIH

DAWD A MEBSTER

NUCLEAR SAFETY ASSOCIATES INC

1OGIPH A LILBERA1AY

JUCLEAR SAFTTY RESEARCH ASSOCIATION

MALLMORI MATMIO

UUCLEAR SYSTEMS ASSOCIATES INC

(HARIES) DIIONA

UUCLEAR WASTE WATCHERS

HFILVIETARTL

NUS CORP

W G BLLTFR

IOSPPHI UMNA $\triangle$ NO

BARRYN N IFT

DOI GLASD ORVIS

DOI GLAS W IONKAY

NWT CORP

U $\perp$ PEARL

OAK RIDGE NATIONAL LABORATORY

H C CLABURNF

ALLFNG CROFI

LESLIL R DOIF

IOHDI ENGSARGER

CAIHYS FORL

DAWDC KOCHLR

ELIIND SMITH

OFFICE OF NWTS INTEGRATION

ROBLRT E HEINL SAY

OHIO ENVIRONMENTAL. COUNCIL

STEPHIN HI GHDAM

OHIO STATE UNIVERSITY

$R \sim$ CMRISTESSE

I A COR $\triangle$ WLLL

OKLAHOMA STATE DEPT OF HEALTH

R I CRAK

ONTARIO HYDRO

C I LIE

(RAIG) SIMPQON

ONTARIO RESEARCH FOUNDATION

IYDIA MI LLCKEVICH

OREGON DEPT OF ENERGY

DONALDU GODARD

ORGANIZATION FOR ECONOMIC

COOPERATION AND DEVELOPMENT

I P OIMIER

PACIFIC NORTHWEST LABORATORY

$$
\begin{aligned}
& 1 \text { BOAVE } \\
& \text { 2) } B R A D I F \\
& \text { BL RKHOLDER } \\
& 1 \text { I CLARK }
\end{aligned}
$$

ORYILLEF HILL

HOYD Y HODGF

J H JARRH II

MAXR KRHILR

DOVALDE LARYON

R D NEISON

R WILLIAM NELSON

RE NIGHUVGALF

R IETF SFRNE

$R$ E NESTLRAAY

I H WESTSIK IR

PARSONS, BRINCKERHOFF, QUADE, \& DOUGLAS, INC.

T C CHEY

$T$ T RLESEL

PB-KBB INC

DHLPS PALL

MARK E SIEINER

PENEERHY ELECTROMELT INTERNATIONAL.

INC

LARRY PENBERTHY

PENNSYLVANIA OFFICE OF VOCATIONAL

REHABULITATION

ANDRH CHOPAK

PENNSYLVANIA STATE UNIVERSITY

WICHAFL GRLTZLCR

WILLIAMI A JESTER

WILLIAM B WHIII

MICHAEL 7OLENSKY

PERRY COUNTY SCHOOLS

IARHEL A COCHRAV

PHYSIKALISCH-TECHNISCHE BUNDESANSTALT

PETER BREQNECKE

POINT BEACH NUCLEAR POWER PLANT

GLEN A RLED

PORTLAND GENERAL ELECTRIC

I I LIAISCH

POWER AUTHORITY OF THE STATE OF NEW YORK

MIROA 1 KACZMARSKY

POWER REACTOR AND NUCLEAR FUEL

DEVELOPMENT CORPORATION

PRESQUE ISLE COURTHOUSE

PRINCETON UNIVERSITY

PFTER MONIAGLE

$G$ I PINDER

PROCESS AND ENGINEERING DEVELOPMENT

GERAIDI RITTER

PUBLIC SERVICE INDIANA

ROBERI S MEGEVG

QUADREX CORP

FRANCKI KENESHILA

RADIAN CORP

BARBARA IIAXEY

RE/SPEC INC

GARYD C ALLAHAN

PALI F W NIRK

REASSELAER POLYIECHANC INSTITUTE

IAATS UL

RIDIHALGH, EGGERS \& ASSOCIATES INC

PHILIP F [GGERS

ROCKWELL HANFORD OPERATIONS

RONALDC ARAETI

HARR BABAD

G S BARAEY

$R$ A DLIL

R J GIMIRA

KARL SI LARLE

SICHAH I SAIIH

K. IHIIRL MALAI

DALCA II R\ER
ROCKWELL INTE GROUP

$n \rightarrow$ PETSETT

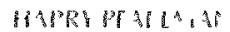

LAVREVII AIITH

ROGERS \& ASSOCIATES ENGINEERING CORP

ARTHI R SL THERLAAD

ROYAL INSTITUTE OF TECHNOLOGY

IARS ALRFTAIRS

ROGERTHL WHK

S.F. LOGAN\& ASSOCIATES INC

STA VLYE LOGAN

S.M. STOLLER CORP

ROBLRT W KUIPP

SAN DIEGO GAS \& ELECTRIC COMPANY $1 O L$ IS BER $\triangle A T H$

SAN JOSE STA IE UNIVERSITY SCHOOL OF

ENGINEEDING

$R \vee A M D L R O A$

SANDIA NATIONAL LABORATORIES

$G C$ ALLFV

RI HLVIFR

IHOAISO III NER

I KETHIOHASOY

O) 1 IONE

$\mathrm{R} W \mathrm{HAH}$

AIARTV A AHECAL

A THOOY ALLLER

I I NOW AR

RICHARD I PLPPING

( I RLDOLTO

GOTI SIN NOCh

4 SNHDFR

I L STEPHENSOA

DANHL AT IALBERT

IYYD TYLR

WE WLLLD WEIRT

WPP CEVTR II HLLS

SAVANNAH RIVER LABORATORY

( $\triangle R O L I A D T F A$

WILISAR ATCDONEL

G $M$ ORE $3 R \& R$

IOH $\triangle A$ STOVE

SCIENCE APPLICATIONS INC

JEIIRLY ARBITAL

IFRR I COHFV

I DOSALD MION

RAIPH HLLLOOD

IAALS HAMANLAIA

RO\ILDHOH MANA

I ROBERTLARIVIIRE

DAWDH IFSTER

PI IFRE MCGRATH

IOHISE MOSULR

RRHHARK WAH

ROBERT I YODLR

SIERRA GEOPHYSICS INC

STLPIII I GHIITT

SIX-COUNTY COMMISSIONERS

ORGANIZATION

C ALCYH H CLTI

SNAKE RIVER ALLIANCE

IAI $\triangle A$ AII!

SOUTH DAKOTA OFFICE OF ENERGY POLIC

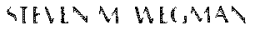

SOUTHWEST RESEARCH AND INFORMATION CENTER

DOS HAPCOCK

ST PONAVENTURE UNIBERSITY

( ARL) TU AROG

ST MARTIN HIGH SCHOOL

R IY AOYDI WERTHALR 
STANFOEO UNUVESITY

AONRADB RRAL QROP!

GFORC, I PARh

IRUVRIASOS

STEA RAS-ROGER SERVICES INC

VERY FCHLV

STORE \& WERSTR ENGINEERIAG CORP

PAIRICIA ANEOCOVWLL

I PORI

FIERTT $A$ TIULR

STURES OVRRBECK \& ASSOCIATES IAC TED E ROLBOH 1

STUDSVIK ENE GITLKAIR A琵

ROLF $\rightarrow O B O D$

SWISS FEDERAL OFFICE OF TAERG I VICDIRIR

SYSTEMS SCIFACE AND SOFIWARE PETER L IGLS

T.M. GATESINC TODD I G YTLS

T.T.I. ENGINEERING CORP DOMAIOC TOMLA

TRCHNICAL INRORMA TION PROJECT DOVILPPAY

TECHNICAL YRESEARCH CENTRE OF HINLAND

OLIII HLIMOYLA

SILIA RI ARIL IVE

MARISAARI

SEPPO IIOKI

TEKNEKMON ONESEARC HANC

A THOWY F1OSC 11

TEXAS A\&M UNIUERSITY

GARY ROBBIY

TFEA BUREAU OF RADATION CONTHOL DONAIDG MDERSOY

TEXAS DEPT OF HEALTH

DAULD K LACRE

TEXAS ENERGY \& NATURAL RESOURCES

ADVISORY COUNCL

TERRY BARROA

CAROL KINC

TEXAS STATE REPRESENTATIVE

PETH LAREY

THE ANALYTIC SCIENCES CORP

JOHN W BARTLETT

CHARLLS 1 KOPLIK

TME CLARION-BEDGER

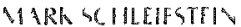

TRW INC

PETRR AII $\triangle A$ A DFR

C. $R$ CHRIGIL

TUN ISMAL ATOMIC RESEARCH CENTRE PUSPAIIIIBRARY

TUSKEGEE INSTITUTE

IRA G DLLIOA

TVO POWER COMPANY

VEIIORYHASE

U.H.D.E.

IRA SK STIRBRI A

U.K. DEPT. OF THE ENU POONAENT

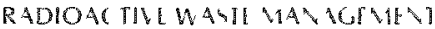
DIVBSION

U.S. ARMY COIES OF ENGINEERS

$A L A Y B L C K$

U.S. BUREAU OF LAND MANAGEMENT

CDUARDR GCHERIC

GREGORYF THAY

U.S. BURCAU OF MANES

CFORGL NEMIADONWL!

U.\$. BUREAU OE RECLAMATION

RECI LEACH
U.\$. DEPT OF ENEEG - AREUQUERUE

OPERATIONS OFFEC

R IONEY

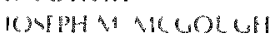

DOR

U.S. DEPT OF ENETEY - ASSIST ANT GENITAL

COUNSEL TOR ENVIRONMENT

- 11 GREL NLEHAH

U.S. DEPT OFEEERE - CHICAGO

OPERATIONS OFPICE

$\mathrm{R}$ OHBY

U.S. DEPT OF ENERGV - DALLAS SUPPORT

OFFICE

CIRILE \& IRISON IR

U.S. DEPT OF ENERGY - DVISION OF WASTE REPOSITORY DEPLOYMENT

IS UAT U HIIARD IR

I It BEn

( R COULEY 3

WARREA KYIR

IIIONISP IOVOOO

HARRS W GLDE

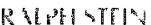

U.S. DEPT OF FNERGY - IDAHO OPERATIONS OFPICE

INIE F LLOR.ARD

$111+3 k 0$

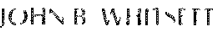

U.S. DEPY OF HERGY - MATTRIALS SCIRPCE DIVISION

R) COTTKRHIII

U.\$. DIPT OF EA ERGY - NEVADA OPERATIOAS

OEFACE

AP KL YRCII

U.5. DERT OF ENERGV - NWTS PROGRAM

OFFICE

I BALLELL

$\checkmark B L A C H A B D$

I A $(A S B)$

R 1 AHOH

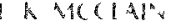

$10 \times 25$

$k k$ hl

R C WLVERLICI

U.S. DEPT OF ENERGY - OFEICF OF WASTE ISOLATION

$106 E \mathrm{PH} A$ IF $A R$

IAVIT SH MHEN

U.S. DEPT OFEAERG - OFEIC OF WAST PRODUCTS

C K OERIL

U.\$. DEPT OF ENERGY - RECHLAND

OPERATIONS OFEICE

R B GORANGOR

D) $\triangle Q$ IRE

U.S. DEPT OE ENERGY - SAVANGAH RIVEH

OPEHATIOAS OFPICE

REUAAT HARR!S

1 H HINDSAA

U.S. DEPT OF ENEMG - WIPY PROCRAM

I ALRIVCIH HARMIOA

U.S. ENVIRONMENTAL PROTECTION AGENCV

DIVISIOA OF C RITIRIA\& TAVLARDS

DONALDHL HTR

I ANTS MHILISR

U.5. GEOLOGICAL SURVIY - COLUMBUS

A 11 A $\triangle A A I R$

U.S. GROLOGICAL SURVEY - DERVER RULARD W ADDEL

U.5. GEOLOGICAL SURVEY - MENLO PARK

IOHIS BREOTHOLTT

MCOBRI BHA
U.5. GEOLOGICAL SURUEY - RESTOW

I $11 \mathrm{Vu}$ C

TOH'. ROBRRTSOR

LUWIA ROLDIUR

II GIVIH ROSGOOOA: HR

PETRR STEDR

DAUIU STI URI

U.5. HOUSE SUPCOMMITTEE ON WERGY AND

THE EPVIRONMENT

1ORRLK LD II

U S. NUCLEAR REGULATOWY COMAASSION

I (ALURB BETOII

R boril

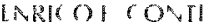

WCHAL C C ILVGFORD

J) Dills

JOSPHI DOAOGHL:

1 i bOMLt

PAI I I OOLOBRR,

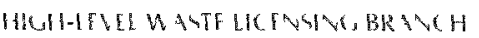

HOH LLIL UASIC TECHVIC

IISOL LCHACAS

$\angle I B R B R$ Y

IANES UAL ARO

IOISB AIRIVI\}

IOHAC MCKIYLE

HL BERT AIILER

R IOHA STARALR

thMiTt + WICK

U.S. SENATE COMMITTEE ON ENERGY AND

NATURAL RESOURCES

MIIISD S SITH

UHDE GMBH

OLMGER

UNC NUCIEAR INLUSTRIES

HD POUE ER

UNION OF CONCERNED SCIENTISTS

MIO HALF ADLN

UNIVERSTTY OF ALABAMA AT IRIRMINGHAM I WAITRR MASOR

UAVERESTY OF ALBERTA

F UL YHWARTI

UNIVERSITY OF ARIZONA

IA AK DAFATE

JAMESC ACCRTH

ROY 4 POST

UNIVERSITY OF CALIFORNIA AT RERKELEV TODO I APORTE

HHONASH PUIOED

UNIVEPSITY OF CALIFORAIA AT LOS ANGELES

D OARTA

UNIVERSITV OF CALIFORNIA AT SAN DIEGO RIIIARIS WILLS

UNIVERSITY OF DELAWARE

FRAVR \& KI I ICR

UNIVERSITY OF RLORLAA

DAYIUE (IARK

DOLORE\& JEQR'S

UNIVERSITY OF ILLINOIS AT URRANA -

CHAMBAIGN

ABBRTI AI MCHELS

UNIVERSITY OF LOWEL

IANESR SHEF

UNIVERSTT OF LULEA

I है $211560 \%$

UNIVESSIT OF MISSOURI AT KANSAS CITY

TDMV D GOLBEL

UNIVYRSTY OF MISSOURI AT ROLLA

ARUIND KI MAR

UNIVEESTTV OF MODERA

$M A 210 \times 1 \triangle 1$

UNIVESITY OF NEVADA AT RENO

BECAY ULISR 
UNIVERSITY OF NEW MEXICO

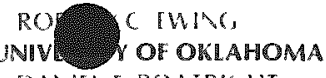

DAVIEL I BO BIRGGHT

UNIVERSTTY OF OTTAWA

II ACER ORIN

UNIVERSITY OF PITTSBURGH

B I COII

UNIVERSITY OF SOUTHERA MISSISSIPPI

CHARILSR BRFNI

IAMES IN PINSON

UNIVERSITY OF TEANESSEE AT KNOXVILLE.

IB II SSELI

UNIVERSTTY OF TEXAS AT AUSTIN

THONASC GI SIALSO

JOE D IIOBU HTE

UNIVERSITY OF TEXAS AT SAN ANIONIO

DONALDR ILM/

UNIVERSITY OF TOKYO

RYONIHI KIYOSL

JNIVERSITY OF UTAH RESEARCH INSTITUTE

[IBRAR\}

INIVERSITY OF WASHINGTON

14 A ROBNIS

INIVERSITY OF WESTERN ONTARIO

WHLIANS HLL

INIVERSITY OF WISCONSIN

B C H MMSON

NIVERSITY OF WISCONSIN AI FOND DU LAC

$1 \mathrm{OH} 、 \mathrm{~B}$ HHIL

INIVERSITY OF WISCONSIN AT MLWAUKEE

HOU ARD PINCL
UTAH BUREAU OF RADIATION CONTROL

DARRIII 11 WARRI

UTAH GEOLOGICAL AND MINEYAL SURVYY

NACI YOYT TAM

UTAH SOUTHEASTERA DHSTRICT HEALTH

DEPARTMENT

ROBLRI L. FLRLOH

IANDEREILT UNIVERSTIY

FRANKI PARKLR

VERMONT STATE NUCLEAR ADVISORY PANLL

VIRGIVIA S ALAA

VIRCINIA DEPT OF HEALTH

ROBERT O WKMIN"

VIRGINA MILITARY INSTITUTE

HE $\backslash R Y D$ Y HRLIBER

VIRGINIA POLYTECHNIC INSTITUTE ANE

STATE UNIVERSITY

HAITHR HIBPARD

DAVIDR WOVES

WASHINGTON DEPT OF SOCIAL AND HEALTH SERVICES

1 STRONC

WASHINGTON HOUSE OF REPRESENTATIUES

RII ISA ACSON
WASHINGION STATE SEAATL

DONACHARMIE

MAVNE SIALE UNGWRSITY

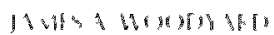

WBAI-FM

UARRE $\triangle 1$ IREOLO

WEST DADE REGIONAL LIBRARY

COLRDLS BL ANOLOPL 7

WEST VALLY NUCEEAR SERUICES COMPANY

INC

RICHARISA WI BR

WEST VIRGINIA GEOLOGICAL AND

ECONOMIC SURVEY

ROBER,B FRMI

WESTINGHOUSE ELECTRIC CORP

GIORGI $\backslash \mathrm{BHAI}$

( AROL A KITS

(1) $\mathrm{ECWB}$

CEORGL $\rightarrow$ SBOL

WESTIVGMUSE WIPP PROIECT

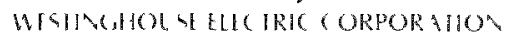

WISCONSIN GEOLOGICAL AND NATURA

MSTORY SURE EY

AICHALI MI DREY IR

WISCONSIN PUBLIC SERVICE CORP

$P \$ 111 O / N_{1}$

WOODMARD-CLVDE CONSULTAATS

SHHOK PAH 3RDH

WP-SYSTEM A

II SR $>$ SGLFOK

WYOMING GEOLOGICAL SUREEY

I) $\triangle 1 H$ A $B U L L R$ 